\title{
Search for the Decay of Neutral B Mesons to a Neutral K Star Meson and a Neutral K Meson Using the BaBar Detector
}

\author{
Stephen David Foulkes \\ Stanford Linear Accelerator Center \\ Stanford University \\ Stanford, CA 94309 \\ SLAC-Report-824 \\ Prepared for the Department of Energy \\ under contract number DE-AC02-76SF00515
}

Printed in the United States of America. Available from the National Technical Information Service, U.S. Department of Commerce, 5285 Port Royal Road, Springfield, VA 22161. 
This document, and the material and data contained therein, was developed under sponsorship of the United States Government. Neither the United States nor the Department of Energy, nor the Leland Stanford Junior University, nor their employees, nor their respective contractors, subcontractors, or their employees, makes an warranty, express or implied, or assumes any liability of responsibility for accuracy, completeness or usefulness of any information, apparatus, product or process disclosed, or represents that its use will not infringe privately owned rights. Mention of any product, its manufacturer, or suppliers shall not, nor is it intended to, imply approval, disapproval, or fitness of any particular use. A royalty-free, nonexclusive right to use and disseminate same of whatsoever, is expressly reserved to the United States and the University. 
SLAC-R-824

June 2006

\title{
UNIVERSITY OF CALIFORNIA RIVERSIDE
}

\author{
Search for the Decay of Neutral B Mesons to a Neutral K Star Meson \\ and a Neutral K Meson Using the BABAR Detector
}
A Dissertation submitted in partial satisfaction of the requirements for the degree of

\section{Doctor of Philosophy}

in

\section{Physics}

by

Stephen David Foulkes

June 2006

Dissertation Committee:

Professor J. William Gary, Chairperson

Professor Owen R. Long

Professor Benjamin C. Shen

Work supported in part by U.S. Department of Energy under contract DE-AC02-76SF00515 
Copyright by

Stephen David Foulkes 2006 
The Dissertation of Stephen David Foulkes is approved:

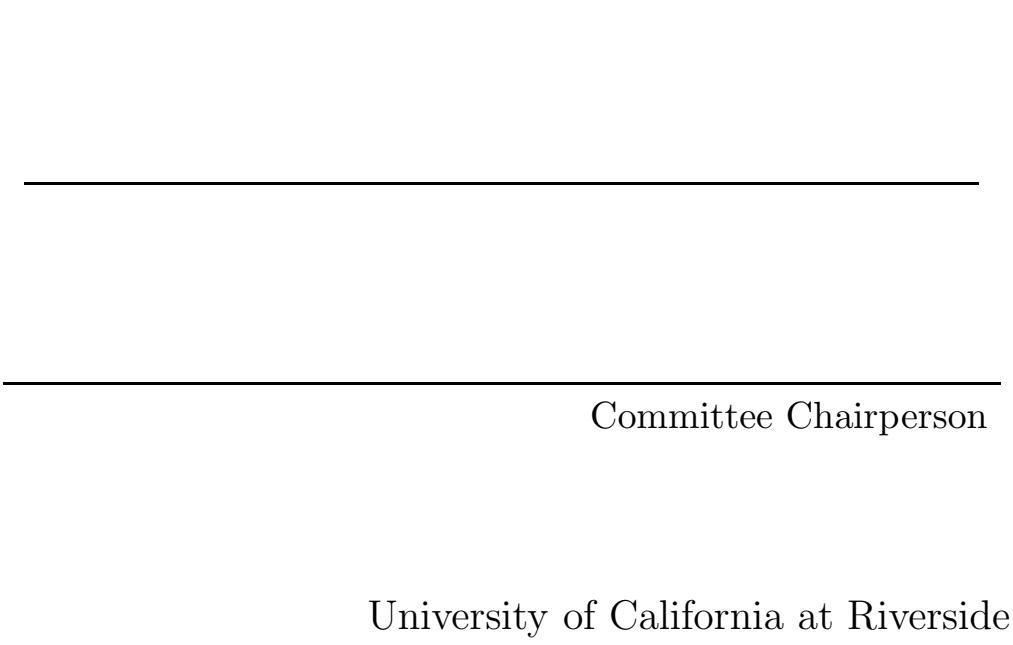




\section{Acknowledgments}

The universe is full of magical things, patiently waiting for our wits to grow sharper.

- Eden Phillpotts

We have a hunger of the mind which asks for knowledge of all around us, and the more we gain, the more is our desire;

the more we see, the more we are capable of seeing.

-Maria Mitchell

I was brought up to believe that the only thing worth doing was to add to the sum of accurate information in the world.

- Margaret Mead

Think deeply of simple things

- Arnold E. Ross

Besides the fact that I find it fun and interesting, these quotes express some of why I pursued this degree and why I continue to pursue physics.

The work contained herein is but a mere speck in the totality of mankind's knowledge, but a speck none the less. This statement does not diminish the work, but enhances it, for knowledge progresses by the sum total of many such specks, and I am proud to have participated in the contribution of this one.

This effort would not have been possible without the help and support of many many people. Let me start with Bill Gary and the UCR group. I would not have gone to UCR if not for Bill Gary. He is a great teacher and has explained many 
of the nuances of particle physics to me (often more than once) with patience and enthusiasm. I could not have had a better adviser. The other members of my faculty committee have also been a tremendous help. The analysis described in this thesis would not have been possible without Owen Long. Owen has been a critical part of every aspect of the analysis from start to finish and provided numerous key insights whenever I got into trouble. I can not over state his contribution. I would also like to thank the other members of my committee: Ben Shen, Bob Clare, and John Baez. While they have not been directly involved in the details of the analysis, their support is certainly appreciated. I would also like to thank the UCR post-docs Kelin Wang and Lei Zhang. Kelin helped me get started at SLAC, and Lei has been invaluable helping with the fit routine and the details of the analysis.

My time at SLAC has been fun, interesting and a great experience. The SLAC staff has been terrific. Richard Boyce, Bob Messner, Jim McDonald, and Karl Bouldin were always quick to respond when I needed help with the IFR gas system. Their wit and humor made the long days working on the BABAR detector a lot more fun. My weekly lunches with Richard and Mike Palrang were a great break from the analysis grind, and I will miss them.

My return to science would not have been possible without Richard W. Colburn. His support, encouragement and overall faith in me were a critical part in the decision to pursue my interest in physics. Without him, none of this would have happened.

Last and most importantly, I would like to thank my wife, Sandy, and my two 
children, Sara and Matt. Their love, support, and encouragement, when most thought I had lost my mind to pursue a $\mathrm{PhD}$, was very much appreciated and has sustained me through some of the rough spots the past 5 years. I could not have done it without them. 


\section{ABSTRACT OF THE DISSERTATION}

Search for the Decay of Neutral B Mesons to a Neutral K Star Meson and a Neutral K Meson Using the BABAR Detector

by

Stephen David Foulkes

Doctor of Philosophy, Graduate Program in Physics

University of California, Riverside, June 2006

Professor J. William Gary, Chairperson

A search for the decay of a $B^{0}$ meson to $K^{* 0} K^{0}$ is presented, using a sample of approximately 230 million $B \bar{B}$ events $\left(210 \mathrm{fb}^{-1}\right.$ of data) collected with the BABAR detector at the PEP-II asymmetric $e^{+} e^{-}$collider at SLAC. I obtain the following upper limit for the branching ratio at $90 \%$ confidence level: $\mathcal{B}\left(B^{0} \rightarrow \bar{K}^{* 0} K^{0}\right)+$ $\mathcal{B}\left(B^{0} \rightarrow K^{* 0} \bar{K}^{0}\right)<1.9 \times 10^{-6}$. The measured branching fraction for the sum of these two modes is $\left(0.2_{-0.8-0.3}^{+0.9+0.1}\right) \times 10^{-6}$. This is the first experimental result for this decay channel. 


\section{Contents}

1 Theory and Motivation 3

1.1 Introduction . . . . . . . . . . . . . . . . . . . 3

1.2 The $B^{0} \rightarrow K^{* 0} K^{0}$ Decay Mode . . . . . . . . . . . . . . . . 4

1.3 Quark Mixing and the CKM Matrix . . . . . . . . . . . 5

1.4 The Unitarity Triangle . . . . . . . . . . . . . . . . . . 99

1.5 Measuring $\sin 2 \boldsymbol{\beta}$ from $\boldsymbol{B}^{\mathbf{0}} \rightarrow \boldsymbol{J} / \boldsymbol{\psi} \boldsymbol{K}_{S}^{\mathbf{0}}$ and $\boldsymbol{B}^{\mathbf{0}} \rightarrow \boldsymbol{\phi} \boldsymbol{K}_{\mathrm{s}}^{\mathbf{0}}$ Modes . . . . . . . . . . . . . . . . . . . . 13

$1.6 \mathrm{SU}(3)$ Flavor Symmetry Relations and SM Pollution . . . . . . . . . 22

1.7 The $\sin 2 \boldsymbol{\beta}$ Discrepancy $\left(\Delta \boldsymbol{S}_{\phi K^{0}}\right) \ldots \ldots \ldots \ldots$

$2 \quad$ PEP-II and the BABAR Detector $\quad 27$

2.1 The LINAC Accelerator and PEP-II Storage Ring at SLAC . . . . . . 27

2.1.1 Design and Performance . . . . . . . . . . . . . 28

2.1.2 Background Radiation and the Machine-Detector Interface (MDI) 32

2.2 Overview of the BABAR Detector . . . . . . . . . . . . . . 33

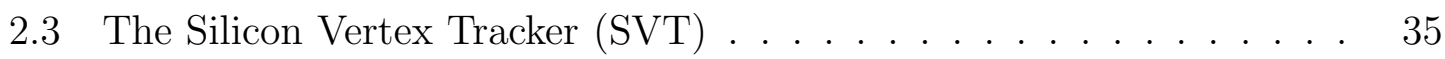

2.3.1 SVT Theory of Operation .............. 35

2.3.2 SVT Design . . . . . . . . . . . . . . . 36

2.3.3 SVT Radiation Monitoring and Protection System (SVTRAD) 39

2.3.4 SVT Performance . . . . . . . . . . . . . . . . . 39

2.4 The Drift Chamber $(\mathrm{DCH}) \ldots \ldots . \ldots . \ldots . . \ldots 43$

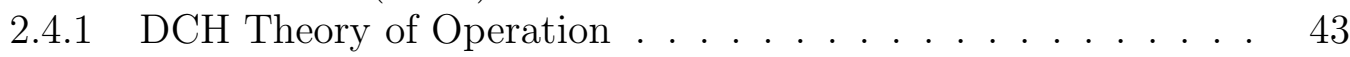

2.4 .2 DCH Design . . . . . . . . . . . . . . . . . 46

2.4 .3 DCH Performance . . . . . . . . . . . . . 46

2.5 The Detector of Internally Reflected Cherenkov light (DIRC) . . . . 51

2.5.1 DIRC Theory of Operation . . . . . . . . . . . . . 51

2.5.2 DIRC Design . . . . . . . . . . . . . . . . . . . . 52

2.5.3 DIRC Performance . . . . . . . . . . . . . . . . 52

2.6 The Electromagnetic Calorimeter (EMC) . . . . . . . . . . . 55

2.6.1 EMC Theory of Operation ............. 55

2.6.2 EMC Design . . . . . . . . . . . . . . . . 55

2.6.3 EMC Performance . . . . . . . . . . . . . . 56 
2.7 The Instrumented Flux Return (IFR) . . . . . . . . . . . . . . 56

2.7.1 IFR Theory of Operation . . . . . . . . . . . . . . . . . . . 59

$2.7 .2 \quad$ IFR Design . . . . . . . . . . . . . . . . . . . . . 62

2.7.3 IFR Performance . . . . . . . . . . . . . . . . . . 63

2.8 The Trigger and Data Acquisition (DAQ) System . . . . . . . . . 63

3 Analysis Techniques $\quad 68$

3.1 Data and Monte Carlo Samples _. . . . . . . . . . . . . . . 68

3.2 Event Selection . . . . . . . . . . . . . . . . . . . . . . . . . . 69

3.2 .1 Pre-selection . . . . . . . . . . . . . . . . . . . . . . . 70

3.2 .2 Particle Identification . . . . . . . . . . . . . . . . . 73

3.2 .3 Fit Window . . . . . . . . . . . . . . . . . . . . 77

$3.2 .4 \quad B^{0}$ Selection Criteria . . . . . . . . . . . . . . . 78

$3.2 .5 \quad K^{* 0}$ Selection Criteria $\ldots \ldots \ldots \ldots$. . . . . . . . 80

$3.2 .6 \quad K_{S}^{0}$ Selection Criteria . . . . . . . . . . . . . . . 80

3.2.7 Event Shape Selection Criteria . . . . . . . . . . . . . . . . . 82

3.2 .8 Optimization of Selection Criteria . . . . . . . . . . . . . . 89

3.2 .9 Multiple $B^{0}$ Candidates . . . . . . . . . . . . . . . . . . 90

3.3 Event Selection Summary _. . . . . . . . . . . . . . . . 90

3.4 Expected Sensitivity . . . . . . . . . . . . . . . . . . . . 92

3.5 Continuum Monte Carlo Evaluation . . . . . . . . . . . . . . . . 93

3.6 Background Evaluation . . . . . . . . . . . . . . . . . . . . . . 94

3.6.1 $B^{0} \bar{B}^{0}$ with the same final state (Category 1 ) . . . . . . . 96

3.6.2 $B^{0} \rightarrow K_{0}^{* 0}(1430) K_{S}^{0}$ channel and other $S$-wave contributions . 99

3.6.3 $B^{0} \bar{B}^{0}$ with a $K_{S}^{0}$ and a Kaon PID Error (Category 2) . . . . 105

3.6.4 $B^{0} \bar{B}^{0}$ with a $K_{S}^{0}$ and a Pion PID Error (Category 3) . . . . 106

3.6.5 $B^{0} \bar{B}^{0}$ Other (Category 4) . . . . . . . . . . . . . . 106

3.6.6 $B^{+} B^{-}$-Related Background . . . . . . . . . . . . . . . . . . 107

3.7 The Extended Maximum Likelihood Fit . . . . . . . . . . . . . . . . 108

3.7.1 Correlations Between Fitted Variables . . . . . . . . . . . 109

3.7.2 Probability Density Functions . . . . . . . . . . . . . . . 110

3.7 .3 Fitted parameters . . . . . . . . . . . . . . . . . . . . . 133

3.8 Fit Validation . . . . . . . . . . . . . . . . . . . . . . . . . . . . . 134

3.8.1 Pure Toy Studies . . . . . . . . . . . . . . . . . . . . 134

3.8.2 Embedded Toy Studies . . . . . . . . . . . . . . . . . . . . 147

3.8.3 Event Shape Selection Criteria Evaluation . . . . . . . . . . 147

3.8.4 Simulated Data Set Studies and Blind Fit . . . . . . . . . 153

4 Results 159

4.1 Maximum Likelihood Fit Results . . . . . . . . . . . . . . . . . . . . 159

4.2 Systematic Uncertainty Estimation . . . . . . . . . . . . . . 166

4.2.1 ML Fit Procedure Uncertainties . . . . . . . . . . . . . . 166 
4.2.2 Efficiency Correction Uncertainties . . . . . . . . . . . . . . . 169

4.2.3 General Systematic Uncertainties and Summary . . . . . . . . 171

$5 \Delta S_{\phi K^{0}}$ Calculation 


\section{List of Tables}

1.1 Theoretical predictions for the $\mathcal{B}_{1}\left(B^{0} \rightarrow \bar{K}^{* 0} K^{0}\right)$ and $\mathcal{B}_{2}\left(B^{0} \rightarrow K^{* 0} \bar{K}^{0}\right)$ branching ratios $\left(\mathcal{B} \times 10^{-6}\right) \ldots \ldots \ldots \ldots \ldots \ldots$

2.1 Accelerator system performance parameters. . . . . . . . . . . . 30

3.1 MC samples used for Signal and $B^{0} \bar{B}^{0}$ background characterization. . 70

3.2 Effect of selection criteria and signal yield. . . . . . . . . . . 71

$3.3 \quad m_{\mathrm{ES}}$ peaking background modes in $B^{0}$ decays. . . . . . . . . . 97

3.4 Correlation coefficients between the three variables used in our fit. "tight" indicates a cut on $m_{\mathrm{ES}}>5.270$ was imposed. . . . . . . . . . 110

3.5 Composition of the MC sample used for Category $1 B \bar{B}$ related background PDF construction. . . . . . . . . . . . . . . . . . . . 123

3.6 Composition of the MC sample used for Category $2 B \bar{B}$ related background PDF construction. . . . . . . . . . . . . . . . . . . . 128

3.7 Composition of the MC sample used for Category $2-\pi B \bar{B}$ related background PDF construction. . . . . . . . . . . . . . . . 129

3.8 Results from the pure toy studies with the Poissonian mean signal and S-wave events (input values) varied between 0 and $15 \ldots . . . . .140$

3.9 Poissonian mean inputs used to study the effect of varying background yields. . . . . . . . . . . . . . . . . . . . . 145

3.10 Results from the pure toy studies with varying Background yields. . . 146

3.11 Results from the embedded toy studies with the embedded number of signal and S-wave $\mathrm{MC}$ events varied between 0 and 15 , and the Poissonian mean number of embedded B background Categories 1, 2, 3 , and 4 fixed at 1, 1, 1, and 56 respectively. Each study consisted of 250 toy samples. The embedded B background Category 4 events were drawn from a total sample of only $217 \mathrm{MC}$ events. . . . . . . . . 148

3.12 The number of signal and generic MC events passing the revised and original Selection Criteria. . . . . . . . . . . . . . . . . 150

4.1 Results. . . . . . . . . . . . . . . . . . . . . . 160

4.2 Percentage systematic uncertainties from varying the Signal PDF parameters and the B background yields that were fixed in the ML fit. . 168

$4.3 K_{S}^{0}$ efficiency correction factors. . . . . . . . . . . . . 170 
4.4 Summary of systematic uncertainties. . . . . . . . . . . . . . 173

5.1 Contributions to the calculation of $\Delta S_{\phi K^{0}}$. The branching ratios and upper limits are from $[48,49] \ldots \ldots$. . . . . . . . . . . . 176 


\section{List of Figures}

1.1 Feynman diagrams for $\bar{B}^{0} \rightarrow K^{* 0} \bar{K}^{0}$. Diagram (a) shows the penguin diagram for this mode and (b) shows a tree level diagram which includes long distance final state re-scattering. . . . . . . . . . 4

$1.2 b$ to $u$ transition vertex. "p" is the four momentum of the $b$ quark, "q" is the four momentum of the $u$ quark, and $k$ is the four momentum of

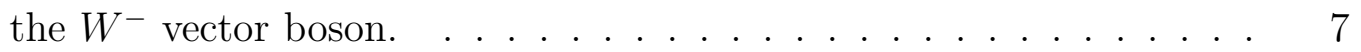

1.3 The Unitarity Triangle. . . . . . . . . . . . . . . . . . . . . . 10

1.4 The Re-scaled Unitarity Triangle in the $\rho \eta$ complex plane. . . . . . 11

1.5 Box diagram for $B^{0} \bar{B}^{0}$ mixing. . . . . . . . . . . . . . 17

1.6 (a) Tree level diagram, (b) penguin diagrams, and (c) virtual $D^{0}$ exchange diagram for the $\bar{B}^{0} \rightarrow J / \psi \bar{K}^{0}$ decay mode. . . . . . . . 18

1.7 (a) Dominant penguin diagrams, (b) suppressed penguin diagram, and (c) virtual kaon exchange diagram for the $\bar{B}^{0} \rightarrow \phi \bar{K}^{0}$ decay mode. . 19

1.8 Current status of $\sin 2 \beta$ from penguin-dominated modes. . . . . . . . 26

2.1 Overview of the LINAC, PEP-II, and the BABAR detector at SLAC. 29

2.2 Integrated luminosity from June 1999 through March 20, 2006 . . . . 31

2.3 Longitudinal section of the BABAR detector. The dimensions shown are in millimeters. . . . . . . . . . . . . . . . . . . . 34

2.4 End view of the BABAR detector. . . . . . . . . . . . . . . 34

2.5 Photo of the completed SVT. . . . . . . . . . . . . . . 38

2.6 Longitudinal schematic drawing of the SVT. . . . . . . . . . . . . 38

2.7 End view of the SVT showing the five layers. . . . . . . . . . 40

2.8 Integrated radiation dose at four locations around the SVT. . . . . . 40

2.9 Z resolution of the SVT in micrometers. . . . . . . . . . . . . 42

2.10 SVT $\phi$ resolution in micrometers. . . . . . . . . . . . . . 42

2.11 Longitudinal section of the BABAR drift chamber. The dimensions are in millimeters. . . . . . . . . . . . . . . . . . . . 48

2.12 Layout of the four innermost layers of the DCH. Lines have been added between the field wires as a visual aide. The "Stereo" numbers on the right side indicate the offset angle (mrad) of the sense wires in that layer for z position measurements. . . . . . . . . . . . . . . . . 49 
2.13 Track reconstruction efficiency for operating voltages of 1900 and 1960 Volts. Tracking efficiency is shown versus the transverse momentum in plot "a" and versus the polar angle in plot "b". . . . . . . . . . .

2.14 The histogram shows the difference between the measured and expected energy loss, $d E / d x$ from Bhabha scattering. The curve is a fitted Gaussian with a resolution of $7.5 \%$. . . . . . . . . . . . . 50

2.15 Schematic of the DIRC imaging system geometry. . . . . . . . . . . 53

2.16 Elevation view of the layout of the DIRC system. . . . . . . . . . . 53

2.17 Efficiency of kaon identification by the DIRC versus track momentum is shown on the top plot. The bottom plot shows the probability that a pion will be mis-identified as a kaon as a function of track momentum. 54

2.18 Schematic of a typical CsI(Tl) crystal (not to scale). . . . . . . . . 57

2.19 Longitudinal cross section of the EMC. All dimensions are in mm. . . 57

2.20 Energy resolution of the EMC for various precesses. . . . . . . . . . . 58

2.21 Angular resolution of the EMC for $\pi^{0} \rightarrow \gamma \gamma \ldots \ldots$. . . . . . . . . . 58

2.22 Overview of the IFR sub-detector. . . . . . . . . . . . . . . 61

2.23 Schematic cross section of an RPC. . . . . . . . . . . . . . 61

2.24 RPC efficiency in the forward end-cap from 1999 through 2004. The top (blue) curve shows the efficiency of only the RPCs that are considered operational. The middle (red) curve shows the average efficiency of all RPCs in the forward end-cap. The bottom (green) curve shows the fraction of inoperative RPCs in the forward end-cap. . . . . . . .

2.25 RPC efficiency in the barrel from 1999 through 2004. The top (blue) curve shows the efficiency of only the RPCs that are considered operational. The middle (red) curve shows the average efficiency of all RPCs in the barrel. The bottom (green) curve shows the fraction of inoperative RPCs in the barrel. . . . . . . . . . . . . . . .

2.26 RPC efficiency in the backward end-cap from 1999 through 2004. The top (blue) curve shows the efficiency of only the RPCs that are considered operational. The middle (red) curve shows the average efficiency of all RPCs in the backward end-cap. The bottom (green) curve shows the fraction of inoperative RPCs in the backward end-cap. . . . . . .

3.1 Sample purity versus $K^{* 0}$ momentum. Plot (a) shows the Kaon purity and plot (b) shows the Pion purity before and after different PID selectors have been applied. The LHTight (solid line) selectors were used in this analysis. . . . . . . . . . . . . . . . . . .

3.2 The kLHTight selector's kaon selection efficiency versus track momen-

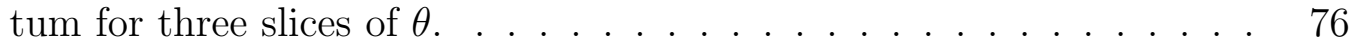

3.3 The piLHTight selector's pion selection efficiency versus track momentum for three slices of $\theta$. . . . . . . . . . . . . . . . . . 76 
3.4 The kLHTight selector's Pion fake rate versus track momentum for three slices of $\theta \ldots \ldots \ldots \ldots \ldots \ldots \ldots$

3.5 The piLHTight selector's kaon fake rate versus track momentum for three slices of $\theta . \ldots \ldots \ldots \ldots \ldots \ldots$

3.6 Plot (a) shows the distribution of $\Delta E$; plot (b) shows the distribution of the $m_{\mathrm{ES}}$; and plot (c) shows the distribution of the invariant mass of the $K^{* 0}$ for the five principal MC samples used in this analysis. Only the pre-selection and fit window selection criteria have been applied. .

3.7 Plot (a) shows the distribution of $B^{0}$ vertex probability and plot (b) shows the distribution of the $\left|\cos \theta_{\text {helicity }}\right|$ for the five principal MC samples used in this analysis. Only the pre-selection and fit window selection criteria have been applied.

3.8 Plot (a) shows the distribution of $K_{S}^{0}$ vertex fit probability and plot (b) shows the distribution of the $K_{S}^{0}$ mass for the five MC samples used in this analysis. Only the pre-selection and fit window selection criteria have been applied. . . . . . . . . . . . . . . .

3.9 Plot (a) shows the distribution of $K_{S}^{0}$ decay length significance and plot (b) shows the distribution of the absolute value of the $K_{S}^{0}$ decay length $\operatorname{Cos}(\theta)$ for the five $\mathrm{MC}$ samples used in this analysis. Only the pre-selection and fit window selection criteria have been applied. . .

3.10 Two dimensional plots of $L_{0}$ versus $L_{2}$. Plot (a) is signal MC events and plot (b) is continuum MC events. The diagonal line is $\mathcal{F}=$ $0.374 L 0-1.179 L 2$, where $\mathcal{F}=0.15$ No selection criteria were applied in these plots. . . . . . . . . . . . . . . .

3.11 Plot (a) shows the distribution of the Fisher discriminant with only the pre-selection and fit window selection criteria applied. Plot (b) shows the distribution of the Fisher discriminant with the cut on the absolute value of cos of the thrust angle. Plot (c) shows the distribution of the absolute value of cos of the thrust angle with only the pre-selection and fit window selection criteria applied. Plot (d) shows the distribution of the absolute value of cos of the thrust angle with the cut on the Fisher discriminant. . . . . . . . . . . . . . .

3.12 Cut optimization plots showing the BF limit vs. each of the variables used as selection criteria. . . . . . . . . . . . . . . .

3.13 Histogram of the number of B candidates per event after all cuts are applied. . . . . . . . . . . . . . . . .

3.14 Comparison of $\mathrm{MC}$ with off-resonance data with (right) and without (left) selection criteria applied. The data points are the off-resonance data and the shaded histograms are the combined $q \bar{q} \mathrm{MC}$ samples. Plots (a) and (b) show the $m_{\mathrm{ES}}$ comparison without and with selection criteria applied respectively; Plots (c) and (d) show the $m_{K s}$ compar-

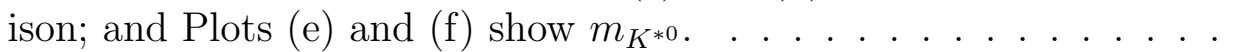


3.15 Virtual W exchange Feynman diagram. . . . . . . . . . . . . . . .

3.16 Efficiency vs. $\operatorname{Cos}\left(\theta_{\text {Helicity }}\right)$. Plots (a) through (d) show the distribution of the $\operatorname{Cos}\left(\theta_{\text {Helicity }}\right)$ for four different regions of $m_{K \pi}$. The black histograms represent the generated MC truth values and the red histograms represent the reconstructed $\mathrm{MC}$ after all cuts have been applied. Plots (e) through (h) show the efficiency distributions for these regions of $m_{K \pi}$.

3.17 Mass vetoes vs. $\operatorname{Cos}\left(\theta_{\text {Helicity }}\right)$. Plot (a) shows the distribution of the $\phi$ invariant mass used to veto $\phi K_{S}$ events versus $\operatorname{Cos}\left(\theta_{\text {Helicity }}\right)$ and the range of the veto (horizontal lines). Plot (b) shows the distribution of the $D K$ invariant mass used to veto $D K$ events versus $\operatorname{Cos}\left(\theta_{\text {Helicity }}\right)$ and the range of the veto (horizontal lines). . . . . . . . . . .

3.18 Signal MC scatter plots of $\Delta E$ vs. $m_{\mathrm{ES}}$ (a), $\Delta E$ vs. the invariant mass of the $K^{* 0}(\mathrm{~b})$, and the $K^{* 0}$ mass vs. $m_{\mathrm{ES}}(\mathrm{c})$. . . . . . . . . . . . . 111

3.19 Category $1 B^{0} \bar{B}^{0}$ background MC scatter plots of $\Delta E$ vs. $m_{\mathrm{ES}}$ (a), $\Delta E$ vs. the invariant mass of the $K^{* 0}(\mathrm{~b})$, and the $K^{* 0}$ mass vs. $m_{\mathrm{ES}}$ (c).

3.20 Category $2 B^{0} \bar{B}^{0}$ background MC scatter plots of $\Delta E$ vs. $m_{\mathrm{ES}}$ (a), $\Delta E$ vs. the invariant mass of the $K^{* 0}(\mathrm{~b})$, and the $K^{* 0}$ mass vs. $m_{\mathrm{ES}}$ (c).

3.21 Category $3 B^{0} \bar{B}^{0}$ background $\mathrm{MC}$ scatter plots of $\Delta E$ vs. $m_{\mathrm{ES}}$ (a), $\Delta E$ vs. the invariant mass of the $K^{* 0}(\mathrm{~b})$, and the $K^{* 0}$ mass vs. $m_{\mathrm{ES}}$ (c).

$3.22 B \bar{B}$ background Category $4 \mathrm{MC}$ scatter plots of $\Delta E$ vs. $m_{\mathrm{ES}}$ (a), $\Delta E$ vs. the invariant mass of the $K^{* 0}(\mathrm{~b})$, and the $K^{* 0}$ mass vs. $m_{\mathrm{ES}}(\mathrm{c})$.

3.23 Continuum background $\mathrm{MC}$ scatter plots of $\Delta E$ vs. $m_{\mathrm{ES}}$ (a), $\Delta E$ vs. the invariant mass of the $K^{* 0}(\mathrm{~b})$, and the $K^{* 0}$ mass vs. $m_{\mathrm{ES}}(\mathrm{c})$. . .

3.24 Signal PDFs. Plot (a) shows the $\Delta E$ for signal MC (data points) and the fitted two Gaussian PDF (solid line). Plot (b) shows the $m_{\mathrm{ES}}$ for signal MC (data points) and the fitted Crystal Ball PDF (solid line). Plot (c) shows the $K^{* 0}$ invariant mass of signal MC (data points) and its fitted Breit-Wigner PDF (solid line).

3.25 Continuum PDFs. Plot (a) shows the $\Delta E$ for continuum MC (data points) and the fitted first order polynomial PDF (solid line). Plot (b) shows the $m_{\mathrm{ES}}$ for continuum MC (data points) and the fitted ARGUS function PDF (solid line). Plot (c) shows the $K^{* 0}$ invariant mass for continuum MC (data points), the fitted first order polynomial plus a Breit-Wigner function PDF (solid line), and the individual components (dashed lines). 
3.26 Peaking $B^{0} \bar{B}^{0}$ Category 1 background PDFs. Plots (a), (b), and (c) show the $\Delta E, m_{\mathrm{ES}}$, and the $m_{K^{* 0}}$ distributions respectively. The data points are the $B^{0} \bar{B}^{0}$ Category 1 modes MC. The solid line is the fitted PDF. The dashed lines are the individual components of the PDF. . 124

$3.27 K_{0}^{* 0}(1430)$ and other non-resonant S-wave $m_{K^{* 0}} \mathrm{PDF} . \quad \ldots \ldots \ldots .126$

3.28 Peaking $B^{0} \bar{B}^{0}$ Category 2 background PDFs. Plots (a), (b), and (c) show the $\Delta E, m_{\mathrm{ES}}$, and the $m_{K^{* 0}}$ distributions respectively. The data points are the $B^{0} \bar{B}^{0}$ Category $2 \mathrm{MC}$. The solid line is the fitted PDF. The dashed lines are the individual components of the PDF. . . . . .

3.29 Peaking $B^{0} \bar{B}^{0}$ Category 3 background PDFs. Plots (a), (b), and (c) show the $\Delta E, m_{\mathrm{ES}}$, and the $m_{K^{* 0}}$ distributions respectively. The data points are the $B^{0} \bar{B}^{0}$ Category $3 \mathrm{MC}$. The solid line is the fitted PDF. The dashed lines are the individual components of the PDF. . . . . .

$3.30 B \bar{B}$ Category 4 PDFs. Plot (a) shows the $\Delta E$ distribution for the Category $4 \mathrm{~B}$ background MC (data points) and the fitted PDF (solid line). Plot (b) shows the $m_{\mathrm{ES}}$ distribution for the Category $4 \mathrm{~B}$ background MC (data points), the fitted PDF (solid line), and the ARGUS and Gaussian components (dashed lines). Plot (c) shows the $m_{K^{* 0}}$ distribution for the Category $4 \mathrm{~B}$ background $\mathrm{MC}$ (data points) and the fitted PDF (solid line).

3.31 Plot (a) shows the distributions of the fitted number of signal events (Nsig). The arrow in plot (a) indicates the value of the Poissonian mean number of signal events. Plot (b) shows the calculated error on the fitted number of signal events (Nsig Error), plot (c) shows the pull as defined in the text, and plot (d) shows the likelihood function for 1,000 pure toy MC samples. . . . . . . . . . . . . . .

3.32 Plot (a) shows the distribution of the fitted number of signal events (Nsig); Plot (b), the calculated error on the fitted number of signal events (Nsig Error); Plot (c) the pull distribution; and Figure 3.32 (d) the likelihood function for 1,000 pure toy MC samples. . . . . . . . 138

3.33 The distribution of the fitted number of signal events (Nsig) for several of the pure toy studies. In plot (a) the Poissonian mean number of signal and S-wave events were set to 3 and 0 respectively. In plot (b) the Poissonian mean number of signal and S-wave events were both set to 3. In plot (c) the Poissonian means were both set to 6 . In plot (d) the Poissonian means were both set to 9. In plot (e) the Poissonian means were both set to 12 . In plot (f) the Poissonian means were both set to $15 . \ldots \ldots \ldots \ldots \ldots \ldots \ldots \ldots$

3.34 Plots (a) and (b) show the pull distributions for S-wave background yield for 1,000 pure toy studies. The Poissonian mean number of signal and S-wave events both set to 3 in plot (a) and 15 in plot (b). . . . 141 
3.35 Plots (a) and (b) show the pull distributions for category $3 \mathrm{~B}$ related background yield with the Poissonian mean number of signal and Swave events both set to 3 and 15 respectively. Plots (c) and (d) show the pull distributions for continuum background yield with the same

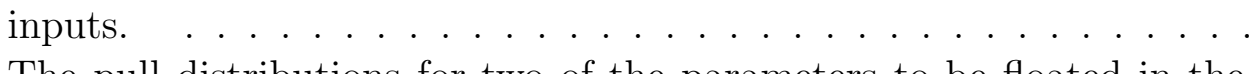

3.36 The pull distributions for two of the parameters to be floated in the continuum background PDFs based on 1,000 pure toy studies. Plots (a) and (b) show the pull distribution for the ARGUS parameter in the $m_{\mathrm{ES}} \mathrm{PDF}$ with the Poissonian mean number of signal and S-wave events both set to 3 and 15 respectively. Plots (c) and (d) show the pull distribution for the polynomial parameter in the $\Delta E$ PDF with the same inputs. . . . . . . . . . . . . . .

3.37 The pull distributions for two of the parameters to be floated in the continuum background PDFs based on 1,000 pure toy studies. Plots (a) and (b) show the pull for the polynomial parameter in the $K^{* 0}$ mass PDF with the Poissonian mean number of signal and S-wave events both set to 3 and 15 respectively. Plots (c) and (d) show the pull distribution for the fraction of the polynomial PDF in the total $K^{* 0}$ mass PDF with the same inputs.

3.38 The distribution of the fitted number of signal events (Nsig). In plot (a) the Poissonian mean number of embedded signal and S-wave events were set to 3 and 0 respectively. In plot (b) the Poissonian mean number of embedded signal and S-wave events were both set to 3 . In plot (c) the Poissonian means were both set to 6 . In plot (d) the Poissonian means were both set to 9. In plot (e) the Poissonian means were both set to 12 . In plot (f) the Poissonian means were both set to

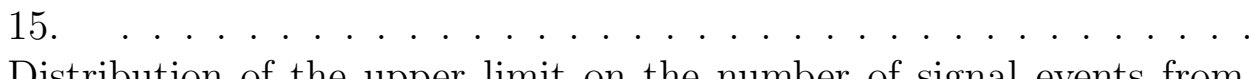

3.39 Distribution of the upper limit on the number of signal events from 200 embedded toy studies using the revised selection criteria. . . . . 152

3.40 Distribution of the upper limit on the number of signal events from 200 embedded toy studies using the original selection criteria. . . . . 152

3.41 Projection plots for the three fitted parameters $\Delta E, m_{\mathrm{ES}}$, and the $K^{* 0}$ mass. Plots (a) and (b) show the $\Delta E$ projection plot with 3 and 15 expected signal events. Plots (c) and (d) show the $m_{\mathrm{ES}}$ projection plot with 3 and 15 expected signal events. Plots (e) and (f) show the $m_{K^{* 0}}$ projection plot with 3 and 15 expected signal events. . . . . . . . .

3.42 Plots of the log likelihood functions for the component PDFs and the product PDF and MC sample. Plots (a) and (c) show the component PDFs with 3 and 15 expected signal events respectively. Plots (b) and (d) show the product PDF the MC sample with 3 and 15 expected signal events respectively. . . . . . . . . . . . . . . 156 
3.43 Plots (a) through (g) compare the distributions of the error for each of the fitted parameters obtained from toy studies (histograms) with the positive and absolute value of the negative error obtained from the blind fit (red arrows). Plot (h) compares the distribution of the minimum Negative Log Likelihood (NLL) value from the toy studies (histogram) with that obtained in the blind fit (red arrow). . . . . .

3.44 Plots (a) through (g) compare the distributions of the global correlations for each of the fitted parameters obtained from toy studies (histograms) with the global correlations obtained from the blind fit (red arrows). . . . . . . . . . . . . . . . . 158

4.1 Projection plots for the three fitted variables $\Delta E, m_{\mathrm{ES}}$, and $m_{K^{* 0}}$. A cut on the likelihood ratio of $>0.6$ was included in all three plots. . . 163

4.2 Log Likelihood Ratio (LLR) plots. Plot (a) shows the LLR for each component PDF and plot (b) shows the LLR for the total PDF and the data sample. . . . . . . . . . . . . . . . . . . . . . 164

$4.3-2 \log \left(\mathcal{L} / \mathcal{L}_{l}\right)$ versus the branching fraction. The dashed green curve is based on statistical uncertainties only, while the solid blue curve includes both statistical and systematic uncertainties. . . . . . . . . 165 


\section{Introduction}

This document describes a search for the decay of a $B^{0}$ meson to a $K^{* 0}$ and a $K^{0}$. Currently, there are no experimental results for this channel. In the Standard Model (SM), this decay is described by the $b \rightarrow d s \bar{s}$ penguin diagram shown in Fig. 1.1. The branching ratio is predicted to be about $0.5 \times 10^{-6}$ [1]-[5]. Throughout this document, the charge conjugate channels are implied unless otherwise stated.

This study also represents the first investigation of a $B^{0}$ decay to a strange vector and strange pseudo-scalar meson, and will provide a test of the branching ratio predictions in [1]-[5]. The analysis assumes that the $K^{* 0}$ decays to a charged kaon and a charged pion and that the $K^{0}$ becomes a $K_{S}^{0}$ and the $K_{S}^{0}$ decays to two charged pions.

The Theory and Motivation chapter describes the portions of the SM as they relate to this particular decay mode. Quark mixing in the $B^{0} \bar{B}^{0}$ system and the CKM matrix are discussed along with recent tests of the CKM model. The current discrepancies that exist between different tests may be due to new physics, higher order effects within the SM (referred to as SM Pollution), or simply statistical fluctuations. The

theoretical basis for how the measurement of the branching fractions for $B^{0} \rightarrow \bar{K}^{* 0} K^{0}$ 
and $B^{0} \rightarrow K^{* 0} \bar{K}^{0}$ can be used, along with several other branching ratios, to put an upper bound on SM Pollution is presented.

Chapter 2 describes the PEP-II accelerator system and the BABAR detector. The design, performance and underlying theoretical basis for each sub-detector is described.

Chapter 3 presents the analysis in detail including the data and MC samples used, event selection procedures, evaluation of background events, the fit procedure, and how the procedure was validated.

Chapter 4 presents the results of the analysis including the estimates of the systematic uncertainties. 


\section{Chapter 1}

\section{Theory and Motivation}

\section{$1.1 \quad$ Introduction}

This chapter describes how the decay of a $B^{0}$ meson to a $K^{* 0}$ meson and a $K^{0}$ meson fits into the overall theory of the weak interactions and why this particular mode is important. The CKM matrix is discussed along with the Unitarity Triangle that summarizes the information relating to the smallest elements of this matrix. The current state of measurements of a portion of the Unitarity Triangle parameterized by $\sin 2 \beta$ are reviewed. Lastly, a theoretical mechanism is described whereby the $B^{0} \rightarrow K^{* 0} K^{0}$ decay mode, along with 9 others, can be used to place an upper bound on the Standard Model (SM) contribution to a possible discrepancy between two different determinations $\sin 2 \beta$. 


\subsection{The $B^{0} \rightarrow K^{* 0} K^{0}$ Decay Mode}

In the Standard Model (SM), the $\bar{B}^{0} \rightarrow K^{* 0} \bar{K}^{0}$ decay is described by the $b \rightarrow d s \bar{s}$ penguin diagram shown in Figure 1.1 with a similar diagram for the conjugate mode $\bar{B}^{0} \rightarrow \bar{K}^{* 0} K^{0}$. Figure 1.1 (a) represents three separate processes wherein the $b$ quark decays into a $d$ quark through emission and absorption of a virtual $W^{ \pm}$and either a virtual $u, c$, or $t$ quark. As will be shown in Section 1.4, the CKM factors for all three of these processes are the same order of magnitude. Figure 1.1 (b) represents a tree level diagram which involves long distance re-scattering to achieve the same final state. The amplitude for this type of process is unknown and therefore may be of the same order as the penguin diagrams. This analysis will measure the sum of all of these diagrams and therefore is it not necessary to attempt to separate them. The SM predictions for the branching ratios for the two components of this decay channel are shown in Table 1.1.

This analysis will measure the sum of these two branching ratios; consequently

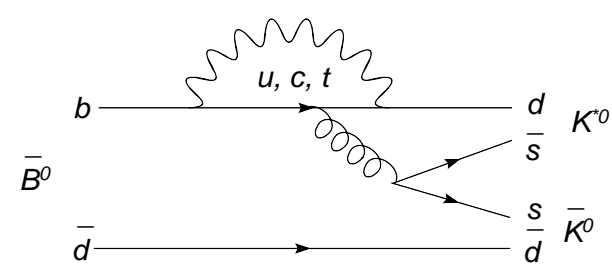

(a)

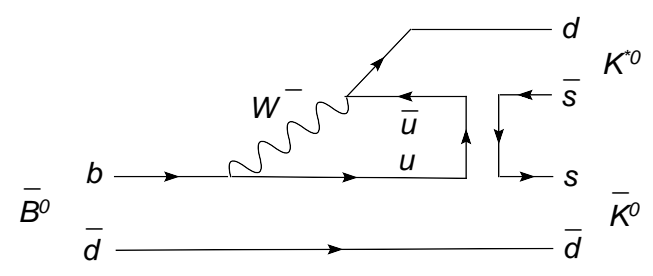

(b)

Figure 1.1: Feynman diagrams for $\bar{B}^{0} \rightarrow K^{* 0} \bar{K}^{0}$. Diagram (a) shows the penguin diagram for this mode and (b) shows a tree level diagram which includes long distance final state re-scattering. 
Table 1.1: Theoretical predictions for the $\mathcal{B}_{1}\left(B^{0} \rightarrow \bar{K}^{* 0} K^{0}\right)$ and $\mathcal{B}_{2}\left(B^{0} \rightarrow K^{* 0} \bar{K}^{0}\right)$ branching ratios $\left(\mathcal{B} \times 10^{-6}\right)$.

\begin{tabular}{|l|c|c|c|}
\hline Author and Year & $\mathcal{B}_{1}$ & $\mathcal{B}_{2}$ & Ref \\
\hline Ali, Kramer, and Lü (1998) & 0.38 & 0.0004 & {$[1]$} \\
Chen, Cheng, Tseng, and Yang (1999) & 0.26 & 0.02 & {$[3]$} \\
Deshpande, Dutta, and Oh (2000) & 0.0281 & 0.00166 & {$[2]$} \\
Beneke and Neubert (2003) & 0.26 & 0.29 & {$[4]$} \\
Chiang, et al. (2003) & 0.46 & 0.61 & {$[5]$} \\
Wang, Lu, Wang, and Yang (2006) & 0.16 & 0.10 & {$[6]$} \\
\hline
\end{tabular}

the theoretical prediction is approximately $1.0 \times 10^{-6}$. If new particles exist beyond the SM, some of these particles could participate in this decay mode as additional loops in the Feynman diagrams. These additional diagrams could result in an anomalously large branching ratio. For example, models incorporating Supersymmetry with R-parity violating interactions estimate that a branching ratio for this mode could be in the range from $5 \times 10^{-9}$ up to $8 \times 10^{-6}[6]$. One of the motivations for this analysis is to eliminate or put an upper limit on branching ratios in the upper end of this range.

\subsection{Quark Mixing and the CKM Matrix}

In the Standard Model the mixing of different quark flavors is, by convention, described in terms of a $3 \times 3$ unitary matrix [7] acting on the $d, s$, and $b$ quarks (the 
mass eigenstates) to form weak eigenstates denoted $d^{\prime}, s^{\prime}$, and $b^{\prime}$.

$$
\left(\begin{array}{c}
d^{\prime} \\
s^{\prime} \\
b^{\prime}
\end{array}\right)=\left(\begin{array}{ccc}
V_{u d} & V_{u s} & V_{u b} \\
V_{c d} & V_{c s} & V_{c b} \\
V_{t d} & V_{t s} & V_{t b}
\end{array}\right)\left(\begin{array}{c}
d \\
s \\
b
\end{array}\right)
$$

Experimentally only left handed quarks couple to the weak interactions. In the Glashow-Weinberg-Salam (GWS) Theory of the weak interactions this is accomplished by assigning the left handed quarks to doublets of $\mathrm{SU}(2)$ and the right handed quarks to singlets under this group. The mixing of quark flavors is incorporated into the theory by including the primed quarks in the bottom half of each doublet.

$$
\left(\begin{array}{l}
u \\
d^{\prime}
\end{array}\right)\left(\begin{array}{l}
c \\
s^{\prime}
\end{array}\right)\left(\begin{array}{l}
t \\
b^{\prime}
\end{array}\right)
$$

By this construction the amplitude for a $b \rightarrow u$ transition like that shown in Figure 1.2 is given by Eq. (1.3) [8]. Similarly, the amplitudes for transitions between other quark flavors are proportional the appropriate CKM matrix element.

$$
\mathrm{i} \mathcal{M}=\frac{i g}{\sqrt{2}} V_{u b} \bar{u}(q) \gamma^{\mu}\left(\frac{1-\gamma^{5}}{2}\right) u(p) \epsilon_{\mu}^{*}(k)
$$

The CKM matrix elements have been measured by several experiments including 


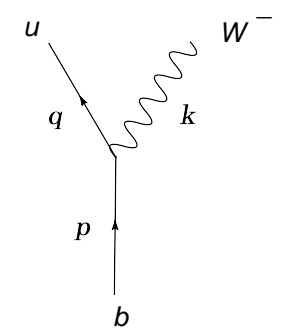

Figure 1.2: $b$ to $u$ transition vertex. "p" is the four momentum of the $b$ quark, "q" is the four momentum of the $u$ quark, and $k$ is the four momentum of the $W^{-}$vector boson.

BABAR and Belle. The current 90\% experimental confidence limits (CL) are [9]

$$
\left(\begin{array}{ccc}
0.9739-0.9751 & 0.221-0.227 & 0.0029-0.0045 \\
0.221-0.227 & 0.9730-0.9744 & 0.039-0.044 \\
0.0048-0.014 & 0.037-0.043 & 0.9990-0.9992
\end{array}\right)
$$

The CKM matrix can be parameterized in a variety of ways, the "standard" parameterization, advocated in Section 11 of [9] and credited to [10], utilizes the mixing angles between the three generations of quarks $\left(\theta_{12}, \theta_{23}, \theta_{13}\right)$ and a phase $(\delta)$. The phase $\delta$ can not be removed by redefining the quark phases. It is this phase that is responsible for $C P$ violation in weak interactions.

This parameterization is generated by combining three standard rotation matrices in the order shown in Eq. (1.5). The phase angle is assigned, by convention, to mixing 
between the first and third generations of quarks.

$$
\begin{aligned}
V= & \left(\begin{array}{ccc}
1 & 0 & 0 \\
0 & c_{23} & s_{23} \\
0 & -s_{23} & c_{23}
\end{array}\right)\left(\begin{array}{ccc}
c_{13} & 0 & s_{13} e^{-i \delta} \\
0 & 1 & 0 \\
-s_{13} e^{-i \delta} & 0 & c_{23}
\end{array}\right)\left(\begin{array}{ccc}
c_{12} & s_{12} & 0 \\
-s_{12} & c_{12} & 0 \\
0 & 0 & 1
\end{array}\right) \\
& =\left(\begin{array}{ccc}
-s_{12} c_{23}-c_{12} s_{23} s_{13} e^{i \delta} & c_{12} c_{23}-s_{12} s_{23} s_{13} e^{i \delta} & s_{23} c_{13} \\
s_{12} s_{23}-c_{12} c_{23} s_{13} e^{i \delta} & -c_{12} s_{23}-s_{12} c_{23} s_{13} e^{i \delta} & c_{23} c_{13}
\end{array}\right)
\end{aligned}
$$

Where $c_{i j}=\cos \theta_{i j}$ and $s_{i j}=\sin \theta_{i j}$ and $\theta_{i j}$ represents the mixing angles between the three generations of quarks.

Wolfenstein [11] proposed a particularly useful approximation of this matrix in terms of four variables: A, $\lambda, \rho$, and $\eta$. Taking $\theta_{13} \approx 0$ reduces the $V_{u s}$ term to the sine of the Cabibbo angle [12]. $V_{u s}$ is currently measured to be $0.2200 \pm 0.0026$ [9]. Wolfenstein proposed to expand the CKM parameters in powers of $V_{u s}=\lambda$ and to set $V_{c b}=A \lambda^{2}$ based on the fact that $\left|V_{c b}\right|$ is currently measured to be $0.0413 \pm 0.0015$ [9], making $A \approx 7 / 8$. When combined with Eq. (1.6) these approximations require $c_{13} \approx 1$ and consequently, to first order, $s_{13} \approx 0 . \quad$ Expanding $c_{12}=\sqrt{1-s_{12}^{2}}=\sqrt{1-\lambda^{2}}$ to order $\lambda^{2}$ yields, $c_{12}=1-\frac{\lambda^{2}}{2}$. With these approximations and keeping only terms of 
order $\lambda^{2}$, Eq. (1.6) becomes

$$
V=\left(\begin{array}{ccc}
1-\lambda^{2} / 2 & \lambda & 0 \\
-\lambda & 1-\lambda^{2} / 2 & A \lambda^{2} \\
0 & -A \lambda^{2} & 1
\end{array}\right)+\mathcal{O}\left(\lambda^{3}\right)
$$

To go to order $\lambda^{3}$, but keeping only leading order terms, the two zero terms in Eq. (1.7) are of the form $A \lambda^{3}$ times some unknown complex number. If the complex number for the $V_{u b}$ term is taken to be $\rho$-i $\eta$, unitarity $\left(V V^{\dagger}=1\right)$ requires the complex number for the $V_{t d}$ term to be $1-\rho-i \eta$ (keeping only terms of order $\lambda^{3}$ ). Putting these into Eq. (1.7) yields the Wolfenstein parameterization to order $\lambda^{3}$.

$$
V=\left(\begin{array}{ccc}
1-\lambda^{2} / 2 & \lambda & A \lambda^{3}(\rho-i \eta) \\
-\lambda & 1-\lambda^{2} / 2 & A \lambda^{2} \\
A \lambda^{3}(1-\rho-i \eta) & -A \lambda^{2} & 1
\end{array}\right)+\mathcal{O}\left(\lambda^{4}\right)
$$

\subsection{The Unitarity Triangle}

The unitarity of the CKM matrix can also be used to create a graphical representation of the complex portions of the matrix. Unitarity applied to the first and third columns yields

$$
V_{u d} V_{u b}^{*}+V_{c d} V_{c b}^{*}+V_{t d} V_{t b}^{*}=0
$$


which contains the relationships between the smallest CKM matrix elements. In the Wolfenstein parameterization $V_{u b}$ and $V_{t d}$ are complex and can be represented in polar form as

$$
\begin{aligned}
& V_{t d}=\left|V_{t d}\right| e^{-i \beta} \\
& V_{u b}=\left|V_{u b}\right| e^{-i \gamma}
\end{aligned}
$$

while the other terms are all real. Equation (1.9) therefore becomes

$$
\left|V_{u d} V_{u b}^{*}\right| e^{+i \gamma}+\left|V_{c d} V_{c b}^{*}\right|+\left|V_{t d} V_{t b}^{*}\right| e^{-i \beta}=0
$$

Note that the angle $\gamma$ enters Eq. (1.11) with a plus sign due to $V_{u b}^{*}$. Each of the three terms in Eq. (1.11) can be represented as a vector in the complex plane, and the requirement that they sum to zero forces them to form a triangle. This triangle (shown in Figure 1.3) is referred to as the "Unitarity Triangle" and is simply a geometrical representation of Eq. (1.9). In $B$ physics the Unitarity Triangle is generally

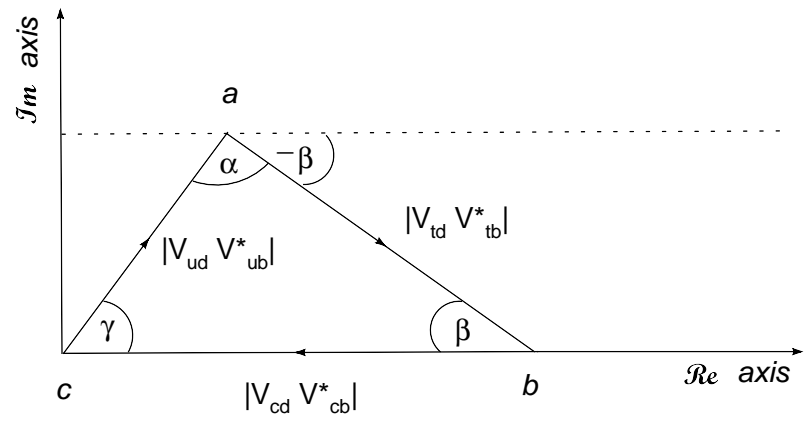

Figure 1.3: The Unitarity Triangle. 
presented using the Wolfenstein parametrization, re-scaled by the base length $V_{c d} V_{c b}^{*}$, and placed with the "c" vertex at the origin. Since $V_{u d} V_{u b}^{*}=\left(1-\frac{\lambda^{2}}{2}\right) A \lambda^{3}(\rho+i \eta)$ and $V_{c d} V_{c b}^{*}=-a \lambda^{3}$, the "b" vertex is located at coordinates $(1,0)$ and the "a" vertex at $(\bar{\rho}, \bar{\eta})$ where $\bar{\rho}=\rho\left(1-\lambda^{2} / 2\right)$ and $\bar{\eta}=\eta\left(1-\lambda^{2} / 2\right)$. Figure 1.4 shows the Unitarity Triangle using this parameterization.

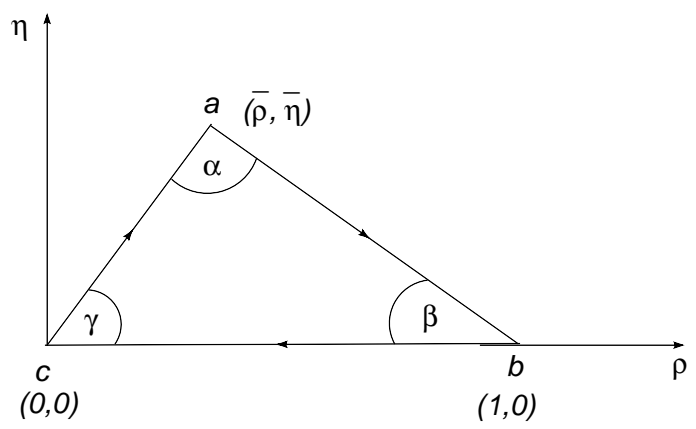

Figure 1.4: The Re-scaled Unitarity Triangle in the $\rho \eta$ complex plane.

Incorporating the parameters $\gamma$ and $\beta$ from the Unitarity Triangle into the Wolfenstein parameterization for the CKM matrix results in a representation particularly suitable for comparison to direct measurements. In this representation

$$
\begin{gathered}
V_{t d}=R_{t} A \lambda^{3} e^{-i \beta} \\
V_{u b}=R_{u} A \lambda^{3} e^{-i \gamma}
\end{gathered}
$$


and the CKM matrix becomes

$$
V=\left(\begin{array}{ccc}
1-\lambda^{2} / 2 & \lambda & A \lambda^{3} R_{u} e^{-i \gamma} \\
-\lambda & 1-\lambda^{2} / 2 & A \lambda^{2} \\
A \lambda^{3} R_{t} e^{-i \beta} & -A \lambda^{2} & 1
\end{array}\right)+\mathcal{O}\left(\lambda^{4}\right)
$$

where $R_{u}=\sqrt{\bar{\rho}^{2}+\bar{\eta}^{2}}$ and $R_{t}=\sqrt{(1-\bar{\rho})^{2}+\bar{\eta}^{2}}$ using the re-scaled Unitarity Triangle (the same formulas apply replacing $\bar{\rho}$ and $\bar{\eta}$ with $\rho$ and $\eta$ if the original Unitarity Triangle is used). The Wolfenstein parameters $\bar{\rho}$ and $\bar{\eta}$ have been measured [9] to be $0.20 \pm 0.09$ and $0.33 \pm 0.05$ respectively, which yields values for $R_{u}$ and $R_{t}$ of 0.39 and 0.87 respectively.

With the CKM matrix in this form the three quark transition amplitudes of interest in the decay mode $B^{0} \rightarrow K^{* 0} K^{0}$ are readily seen to be of the same order of magnitude in powers of $\lambda$.

$$
\begin{aligned}
V_{u d} V_{u b}^{*} & =\left(1-\lambda^{2} / 2\right)\left(A \lambda^{3} R_{u} e^{-i \gamma}\right) \\
V_{c d} V_{c b}^{*} & =-\lambda\left(A \lambda^{2}\right) \\
V_{t d} V_{t b}^{*} & =A \lambda^{3} R_{t} e^{-i \beta}(1)
\end{aligned}
$$

Consequently all three diagrams shown in fig. 1.1a are expected to make roughly equal contributions to the total cross section. 


\subsection{Measuring $\sin 2 \beta$ from $B^{0} \rightarrow J / \psi K_{S}^{0}$ and $B^{0} \rightarrow \phi K_{S}^{0}$ Modes}

One of the principal objectives of the BABAR and Belle experiments is to make precision measurements of the three angles of the Unitarity Triangle in order to test the validity of the CKM matrix model of quark mixing. This analysis relates to the measurement of $\sin 2 \beta$, or more specifically, to the difference between the measurements

of $\sin 2 \beta$ using the $B^{0} \rightarrow J / \psi K_{S}^{0}$ and $B^{0} \rightarrow \phi K_{S}^{0}$ modes. This section summarizes the theory regarding these measurements.

$\operatorname{Sin} 2 \beta$ can only be measured using modes that exhibit a $C P$ asymmetry, for example, where the probability for the decay of a particle and its antiparticle to the same final state are not equal. A standard measure of this asymmetry in the $B^{0} \bar{B}^{0}$ system is given by the following

$$
A_{c p}^{f}(t)=\frac{P\left(\bar{B}^{0} \rightarrow f ; t\right)-P\left(B^{0} \rightarrow f ; t\right)}{P\left(\bar{B}^{0} \rightarrow f ; t\right)+P\left(B^{0} \rightarrow f ; t\right)}
$$

where $P\left(B^{0} \rightarrow f ; t\right)$ represents the probability that a $B^{0}$ will decay to the state $f$ at time $t$. These probabilities are given by the square of the amplitude for the decay. The amplitudes are denoted $A_{f}$ for the particle decay and $\bar{A}_{f}$ for the conjugate decay.

The $B^{0}$ and the $\bar{B}^{0}$ states are known to mix. Consequently, the flavor eigenstates are not the mass eigenstates. The mass eigenstates are generally denoted $B_{H}$ and $B_{L}$ to distinguish the heavy state from the light state. The mass eigenstates are linear 
combinations of the flavor eigenstates with complex coefficients to allow for a phase difference between the two states:

$$
\begin{aligned}
& \left|B_{L}\right\rangle=p\left|B^{0}\right\rangle+q\left|\bar{B}^{0}\right\rangle \\
& \left|B_{H}\right\rangle=p\left|B^{0}\right\rangle-q\left|\bar{B}^{0}\right\rangle
\end{aligned}
$$

where $p$ and $q$ are complex and $|p|^{2}+|q|^{2}=1$. The Schrödinger equation is used to separate out the time dependence of $B_{H}$ and $B_{L}$ which gives

$$
\begin{gathered}
\left|B_{L}(t)\right\rangle=e^{-i m_{L} t} e^{-\Gamma_{L} t / 2}\left|B_{L}(0)\right\rangle \\
\left|B_{H}(t)\right\rangle=e^{-i m_{H} t} e^{-\Gamma_{H} t / 2}\left|B_{H}(0)\right\rangle
\end{gathered}
$$

The particles are produced as flavor eigenstates so it is necessary to solve Eq. (1.16) for $\left|B^{0}\right\rangle$ and $\left|\bar{B}^{0}\right\rangle$.

$$
\begin{aligned}
& \left|B^{0}\right\rangle=\frac{1}{2 p}\left(\left|B_{L}\right\rangle+\left|B_{H}\right\rangle\right) \\
& \left|\bar{B}^{0}\right\rangle=\frac{1}{2 q}\left(\left|B_{L}\right\rangle-\left|B_{H}\right\rangle\right)
\end{aligned}
$$

The probability for a given decay to occur is given by

$$
P\left(B^{0} \rightarrow f ; t\right)=\left|\left\langle f|H| B^{0}(t)\right\rangle\right|^{2}
$$

The equations 1.16 through 1.19 can be combined to yield equations for the probabilities in terms of $p, q$, and the the matrix elements $\left\langle f|H| B^{0}\right\rangle$ and $\left\langle f|H| \bar{B}^{0}\right\rangle$ which define 
the two amplitudes $A_{f}$ and $\bar{A}_{f}$ respectively, where $B^{0}$ and $\bar{B}^{0}$ decay to a common final state. The terms needed to calculate the probabilities are then

$$
\begin{aligned}
& \left\langle f|H| B^{0}(t)\right\rangle=\frac{1}{2}\left[e^{-i m_{L} t} e^{-\Gamma_{L} t / 2}\left(A_{f}+\frac{q}{p} \bar{A}_{f}\right)+e^{-i m_{H} t} e^{-\Gamma_{H} t / 2}\left(A_{f}-\frac{q}{p} \bar{A}_{f}\right)\right] \\
& \left\langle f|H| \bar{B}^{0}(t)\right\rangle=\frac{1}{2}\left[e^{-i m_{L} t} e^{-\Gamma_{L} t / 2}\left(\frac{q}{p} A_{f}+\bar{A}_{f}\right)-e^{-i m_{H} t} e^{-\Gamma_{H} t / 2}\left(\frac{q}{p} A_{f}-\bar{A}_{f}\right)\right]
\end{aligned}
$$

To simplify these equations $\Gamma_{H}$ and $\Gamma_{L}$ can be replaced by $\Gamma$ because the decay rates for $\left|B_{H}\right\rangle$ and $\left|B_{L}\right\rangle$ are nearly identical and $\lambda_{f}$ and $\Delta m$ are generally defined by the following:

$$
\lambda_{f} \equiv \frac{q}{p} \frac{\bar{A}_{\bar{f}}}{A_{f}} \quad \text { and } \quad \Delta m \equiv m_{H}-m_{L}
$$

With these definitions the probability $\left(B^{0} \rightarrow f ; t\right)$ is given by

$$
\begin{aligned}
\left|\left\langle f|H| B^{0}(t)\right\rangle\right|^{2}= & \frac{1}{4} e^{-\Gamma t}\left|A_{f}\right|^{2}\left\{1+\left|\lambda_{f}\right|^{2}+\frac{p}{q} \lambda_{f}\left(\frac{q^{*}}{p^{*}}+\frac{q}{p}\right)+1+\left|\lambda_{f}\right|^{2}-\frac{p}{q} \lambda_{f}\left(\frac{q^{*}}{p^{*}}+\frac{q}{p}\right)\right. \\
& \left.+e^{i \Delta m t}\left[1-\left|\lambda_{f}\right|^{2}+\left(\lambda_{f}-\lambda_{f}^{*}\right)\right]+e^{-i \Delta m t}\left[1-\left|\lambda_{f}\right|^{2}-\left(\lambda_{f}-\lambda_{f}^{*}\right)\right]\right\}
\end{aligned}
$$

with a similar equation for $\bar{B}^{0}$. The two $\frac{p}{q} \lambda_{f}\left(\frac{q^{*}}{p^{*}}+\frac{q}{p}\right)$ terms in the first line of Eq. $(1.22)$ cancel and $\lambda_{f}-\lambda_{f}^{*}=2 i \mathcal{I} m \lambda_{f}$. With these simplifications and expressing the exponentials in Eq. (1.22) in terms of sines and cosines yields the following for the two probabilities

$$
\begin{aligned}
& \left|\left\langle f|H| B^{0}(t)\right\rangle\right|^{2}=\frac{1}{2} e^{-\Gamma t}\left|A_{f}\right|^{2}\left\{1+\left|\lambda_{f}\right|^{2}+\left(1-\left|\lambda_{f}\right|^{2}\right) \cos (\Delta m t)-2 \mathcal{I} m \lambda_{f} \sin (\Delta m t)\right\} \\
& \left|\left\langle f|H| \bar{B}^{0}(t)\right\rangle\right|^{2}=\frac{1}{2} e^{-\Gamma t}\left|\bar{A}_{f}\right|^{2}\left\{1+\frac{1}{\left|\lambda_{f}\right|^{2}}+\left(1-\frac{1}{\left|\lambda_{f}\right|^{2}}\right) \cos (\Delta m t)-\frac{2 \mathcal{I} m \lambda_{f}}{\left|\lambda_{f}\right|^{2}} \sin (\Delta m t)\right\} .
\end{aligned}
$$


Putting these into Eq. (1.15) for $A_{c p}^{f}(t)$, dividing the numerator and denominator by $\left|A_{f}\right|^{2}$, and noting from Eq. (1.21) that

$$
\frac{\left|\bar{A}_{f}\right|^{2}}{\left|A_{f}\right|^{2}}=\left|\lambda_{f}\right|^{2} \frac{|p|^{2}}{|q|^{2}}
$$

the asymmetry becomes

$$
A_{c p}^{f}(t)=\frac{-\left(1+\left|\lambda_{f}\right|^{2}\right)\left(1-\frac{|p|^{2}}{|q|^{2}}\right)+\left(1+\frac{|p|^{2}}{||^{2}}\right)\left\{-\left(1-\left|\lambda_{f}\right|^{2}\right) \cos (\Delta m t)+2 \mathcal{I} m \lambda_{f} \sin (\Delta m t)\right\}}{\left(1+\left|\lambda_{f}\right|^{2}\right)\left(1+\frac{|p|^{2}}{|q|^{2}}\right)+\left(1-\frac{|p|^{2}}{|q|^{2}}\right)\left\{\left(1-\left|\lambda_{f}\right|^{2}\right) \cos (\Delta m t)-2 \mathcal{I} m \lambda_{f} \sin (\Delta m t)\right\}} .
$$

Making the approximation that $\frac{|p|^{2}}{|q|^{2}} \approx 1$ (an approximation that is good to within less than a percent [9], sec.12.6) eliminates the first term in the numerator and the second term in the denominator and greatly simplifies this equation. With this approximation $A_{c p}^{f}$ takes the relatively simple form of

$$
A_{c p}^{f}=S_{f} \sin (\Delta m t)-C_{f} \cos (\Delta m t)
$$

where

$$
S_{f} \equiv \frac{2 \mathcal{I} m\left(\lambda_{f}\right)}{1+\left|\lambda_{f}\right|^{2}}, \quad \text { and } \quad C_{f} \equiv \frac{1-\left|\lambda_{f}\right|^{2}}{1+\left|\lambda_{f}\right|^{2}}
$$

Even though the ratio $\frac{|q|}{|p|}$ is very close to unity the phase difference in the form of $e^{-i \phi_{M(B)}}$ must still be included in $\lambda_{f}$ (the notation " $\mathrm{M}(\mathrm{B})$ " is in reference to the mass matrix of the Hamiltonian for $B$ mixing). The relative phase comes from the box diagram for $B^{0} \bar{B}^{0}$ mixing shown in Fig. 1.5. The relative phase factor for $B^{0} \bar{B}^{0}$ 


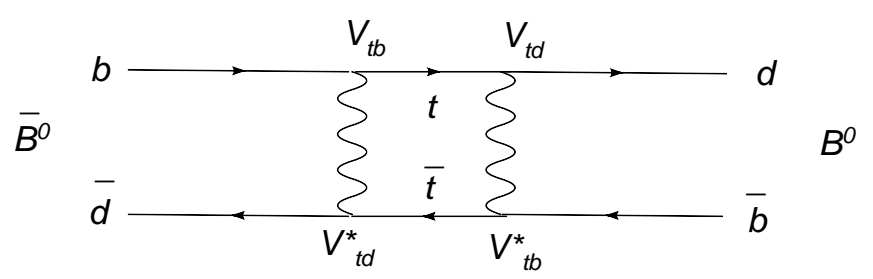

Figure 1.5: Box diagram for $B^{0} \bar{B}^{0}$ mixing.

mixing is therefore given by

$$
e^{-i \phi_{M(B)}}=\frac{V_{t b}^{*} V_{t d}}{V_{t b} V_{t d}^{*}}
$$

In the notation of the Unitarity Triangle and using the Wolfenstein parameterization of the CKM matrix the relative phase angle, $\phi_{M(B)}=2 \beta$.

Of particular interest are the modes $f=J / \psi K^{0}$ and $f=\phi K^{0} . B^{0} \rightarrow J / \psi K^{0}$ is called the "Golden Mode" and is considered the cleanest mode to measure $\sin 2 \beta$ and was the first mode used to test the validity of the mechanism for $C P$ violations in $B$ decays in the CKM matrix model. $B^{0} \rightarrow \phi K^{0}$ and other charmless modes have also been used to measure $\sin 2 \beta$. However, the values are not fully in agreement with the charmonium modes (See Sect. 1.7). One of the motivations for this analysis is to provide data to better understand the discrepancy.

The $J / \psi K^{0}$ mode is a $\bar{b} \rightarrow \bar{c} c \bar{s}$ transition and proceeds via a tree level Feynman diagram, penguin diagrams, or virtual $D^{0}$ exchange involving long distance re-scattering. These diagrams are shown in Figures 1.6 (a), (b), and (c) respectively. The amplitudes for the tree level diagram and two of the penguins are of order $\lambda^{2}$ (this is the Wolfenstein parameter and must not be confused with $\lambda_{f}$ defined in 1.21). The 


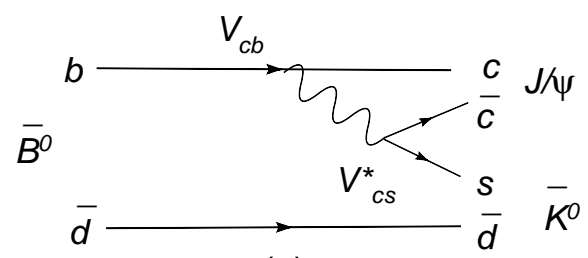

(a)

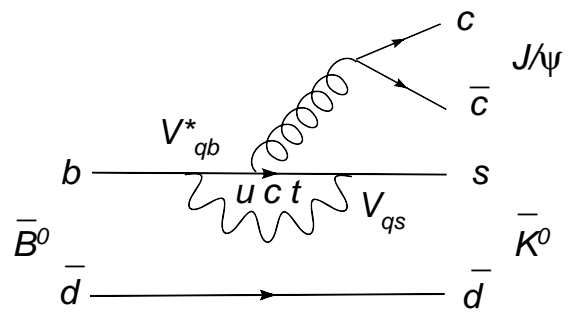

(b)

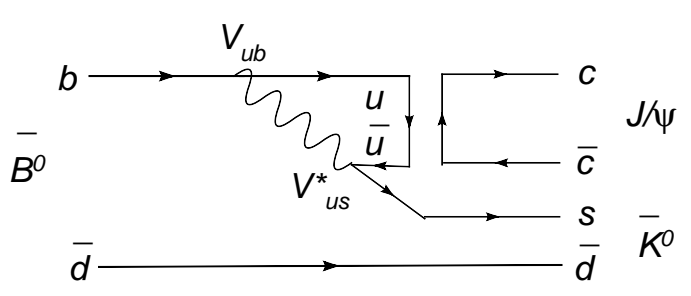

(c)

Figure 1.6: (a) Tree level diagram, (b) penguin diagrams, and (c) virtual $D^{0}$ exchange diagram for the $\bar{B}^{0} \rightarrow J / \psi \bar{K}^{0}$ decay mode.

third penguin with the $u$ quark and the virtual $D^{0}$ exchange diagram are suppressed by an additional factor of $\lambda^{2}$.

The $\phi K^{0}$ mode is a $\bar{b} \rightarrow \bar{s} s \bar{s}$ transition and proceeds via penguin diagrams or through a three-level diagram with re-scattering. The Feynman diagrams for the $\phi K^{0}$ mode are shown in Figure 1.7. The amplitudes for two of the penguins are of order $\lambda^{2}$ while the third penguin (the $u$ quark) and the virtual kaon exchange diagrams are suppressed by an additional factor of $\lambda^{2}$. In addition the third penguin contains the CKM phase factor $\gamma$.

The amplitudes for the two decay modes are simply the sum of the individual diagrams including a possible relative weak phase difference between each diagram. The contributions from the virtual $D$ and $K$ exchange diagrams have been absorbed 


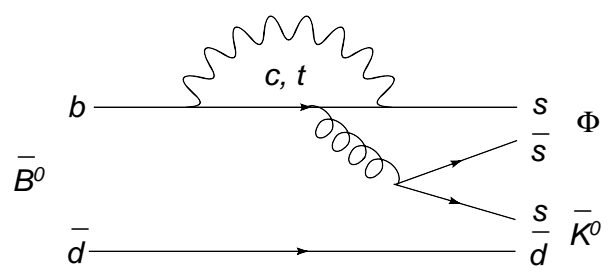

(a)

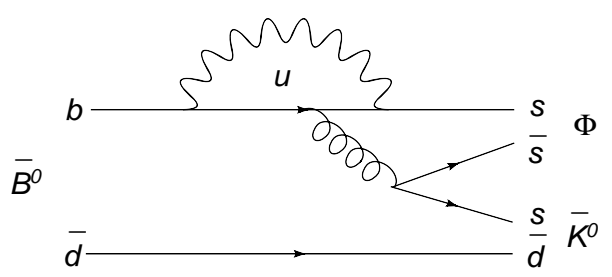

(b)

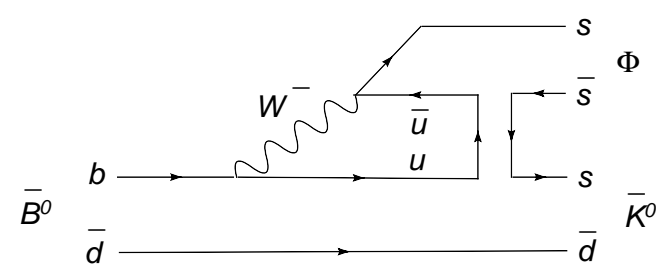

(c)

Figure 1.7: (a) Dominant penguin diagrams, (b) suppressed penguin diagram, and (c) virtual kaon exchange diagram for the $\bar{B}^{0} \rightarrow \phi \bar{K}^{0}$ decay mode.

into the $u$ quark penguin diagrams as they contain the same $V_{u b}^{*} V_{u s}$ term.

$$
\begin{aligned}
& A_{\psi K}=\left(V_{c b}^{*} V_{c s}\right) t_{\psi K}+\left(V_{u b}^{*} V_{u s}\right) p_{\psi K}^{u}+\left(V_{c b}^{*} V_{c s}\right) p_{\psi K}^{c}+\left(V_{t b}^{*} V_{t s}\right) p_{\psi K}^{t} \\
& A_{\phi K}=\left(V_{u b}^{*} V_{u s}\right) p_{\phi K}^{u}+\left(V_{c b}^{*} V_{c s}\right) p_{\phi K}^{c}+\left(V_{t b}^{*} V_{t s}\right) p_{\phi K}^{t}
\end{aligned}
$$

Where $\arg \left(t_{\psi K}\right)$ and the $\arg \left(p_{f}^{i}\right)$ are the relative weak phases of the tree and penguin diagrams respectively from the CKM matrix terms.

The factors in Eq. (1.27) are not independent due to the unitarity of the CKM matrix. Consequently, one of the terms can be eliminated but the relative phase must be retained. Applying unitarity to the second and third columns of the CKM matrix (Eq. (1.1) yields

$$
V_{u b}^{*} V_{u s}+V_{c b}^{*} V_{c s}+V_{t b}^{*} V_{t s}=0 .
$$


Using Eq. (1.28) to eliminate $V_{t b}^{*} V_{t s}$ from Eq. (1.27) and collecting terms yields

$$
\begin{aligned}
& A_{\psi K}=\left(V_{c b}^{*} V_{c s}\right)\left(t_{\psi K}+p_{\psi K}^{c}-p_{\psi K}^{t}\right)+\left(V_{u b}^{*} V_{u s}\right)\left(p_{\psi K}^{u}-p_{\psi K}^{t}\right) \\
& A_{\phi K}=\left(V_{c b}^{*} V_{c s}\right)\left(p_{\phi K}^{c}-p_{\phi K}^{t}\right)+\left(V_{u b}^{*} V_{u s}\right)\left(p_{\phi K}^{u}-p_{\phi K}^{t}\right) .
\end{aligned}
$$

Putting this into Eq. (1.21) for $\lambda_{f}$ and using Eq. (1.26) for $q / p$ yields

$$
\begin{aligned}
& \lambda_{\psi K}=e^{-i 2 \beta} \times \frac{\left(V_{c b} V_{c s}^{*}\right) a_{\psi K}^{t r}+\left(V_{u b} V_{u s}^{*}\right) a_{\psi K}^{u}}{\left(V_{c b}^{*} V_{c s}\right) a_{\psi K}^{t}+\left(V_{u b}^{*} V_{u s}\right) a_{\psi K}^{u}} \\
& \lambda_{\phi K}=e^{-i 2 \beta} \times \frac{\left(V_{c c} V_{c s}^{*}\right) a_{\phi K}^{c}+\left(V_{u b} V_{u s}^{*}\right) a_{\phi K}^{u}}{\left(V_{c b}^{*} V_{c s}\right) a_{\phi K}^{c}+\left(V_{u b}^{*} V_{u s}\right) a_{\phi K}^{u}}
\end{aligned}
$$

where $a_{\psi K}^{t r}=t_{\psi K}+p_{\psi K}^{c}-p_{\psi K}^{t}, \quad a_{\phi K}^{c}=p_{\phi K}^{c}-p_{\phi K}^{t}$, and $a_{f}^{u}=p_{f}^{u}-p_{f}^{t}$.

Dividing the numerators and denomiators by $V_{c b} V_{c s}^{*}$ and using the Wolfenstein parameterization for the CKM matrix elements, Eq. 1.30 becomes

$$
\begin{aligned}
& \lambda_{\psi K}=e^{-i 2 \beta} \times \frac{a_{\psi K}^{t r}+\left(\lambda^{2} R_{u} e^{-i \gamma}\right) a_{\psi K}^{u}}{a_{\psi K}^{t r}+\left(\lambda^{2} R_{u} e^{+i \gamma}\right) a_{\psi K}^{u}} \\
& \lambda_{\phi K}=e^{-i 2 \beta} \times \frac{a_{\phi K}^{c}+\left(\lambda^{2} R_{u} e^{-i \gamma}\right) a_{K K}^{u}}{a_{\phi K}^{c}+\left(\lambda^{2} R_{u} e^{+i \gamma}\right) a_{\phi K}^{\psi}}
\end{aligned}
$$

For the two modes being considered here it is also necessary to consider mixing in the final state. The $K^{0}$ and $\bar{K}^{0}$ mix and this fact requires an additional multiplicative phase similar to Eq. (1.26) for the $B^{0} \bar{B}^{0}$ mixing. In the SM the phase factor for $K^{0} \bar{K}^{0}$ mixing is

$$
e^{-i \phi_{M(K)}}=\frac{V_{c d}^{*} V_{c s}}{V_{c d} V_{c s}^{*}}
$$

To order $\lambda^{3}$ in the Wolfenstein parameterization $V_{c d}$ and $V_{c s}$ are both real and there- 
fore this phase factor is equal to one. For orders of $\lambda^{4}$ and higher $K^{0} \bar{K}^{0}$ mixing introduces an additional phase factor that must be included in the equation for $\lambda_{f}$.

In the $B^{0} \rightarrow J / \psi K^{0}$ decay channel there is a tree level diagram that has the same phase as the penguin diagrams with $c$ and $t$ quarks in the loop (up to corrections of order $\left.\arg \left(\frac{V_{c s} V_{c b}^{*}}{V_{t s} V_{t b}^{*}}\right)\right)$ [16]. $\quad a_{\psi K}^{t r} \gg a_{\psi K}^{u}$ and therefore $a_{\psi K}^{u}$ can be neglected in Eq. (1.30) to an approximation that is better than one percent (see [9], sec. 12.6 and $[17])$. With this approximation, the numerator and denominator in Eq. (1.30) will cancel to $\mathcal{O}\left(\lambda^{3}\right)$ because $V_{c b}$ and $V_{c s}$ are both real. The $J / \psi K_{S}^{0}$ mode, therefore, cleanly measures $\sin 2 \beta$.

Similarly, to the extent that the $a_{\phi K}^{u}$ term in Eq. (1.30) can be neglected, the $\phi K_{S}^{0}$ mode will also directly measure $\sin 2 \beta$. However, since the leading terms of $B^{0} \rightarrow \phi K_{S}^{0}$ begin at one-loop order it is expected that $a_{\phi K}^{c} \sim a_{\phi K}^{u}$ [17]. Setting $a_{\phi K}^{c}=a_{\phi K}^{u}$ in Eq. (1.30) results in an additional factor of $\mathcal{O}\left(\lambda^{2}\right)$ (roughly 5\%) in the determination of $\lambda_{\phi K}$.

The long distance re-scattering effects (see, for example, Fig. 1.7) could also affect the determination of $\lambda_{\phi K}$, but they are believed to be small [17]. Including the $a_{\phi K}^{u}$ term in Eq. (1.30) adds what is termed "Standard Model Pollution" in the $\sin 2 \beta$ measurement using the $\phi K_{S}^{0}$ channel.

The evaluation of SM pollution generally utilizes the parameter $\xi_{\phi K}$ defined by

$$
\xi_{\phi K} \equiv \frac{V_{u b}^{*} V_{u s} a_{\phi K}^{u}}{V_{c b}^{*} V_{c s} a_{\phi K}^{c}}
$$


The amplitude can then be rewritten in terms of $\xi_{\phi K}$ as

$$
A_{\phi K}=V_{c b}^{*} V_{c s} a_{\phi K}^{c}\left(1+\xi_{\phi K}\right)
$$

To first order in the variable $\left|\xi_{\phi K}\right|$ the difference between $\sin 2 \beta$ as measured by the $J / \psi K_{S}^{0}$ mode and that measured by the $\phi K_{S}^{0}$ mode $\left(\Delta S_{\phi K^{0}}\right)$ is given by $[14,15,16]$

$$
\Delta S_{\phi K^{0}}=2\left|\xi_{\phi K}\right| \cos 2 \beta \sin \gamma \cos \delta_{\phi K}
$$

where $\delta_{\phi K}=\arg \left(a_{\phi K}^{u} / a_{\phi K}^{c}\right)$. Unfortunately, $\xi_{\phi K}$ and $\delta_{\phi K}$ can not be calculated in a model independent manner.

\subsection{SU(3) Flavor Symmetry Relations and SM Pol- lution}

Grossman et al. [15] introduced a method to obtain a SM bound on $\Delta S_{\phi K^{0}}$, using $\mathrm{SU}(3)$ flavor symmetry to relate the SM pollution terms in Fig. 1.7 (b) and (c) to strangeness conserving, penguin-dominated processes such as those shown in Fig. 1.1 for $B^{0} \rightarrow K^{* 0} K^{0}$. The method relies on the following relation [15] between the $B^{0} \rightarrow \phi K^{0}$ decay amplitude, $a\left(\phi K^{0}\right)$, and strangeness conserving decay amplitudes, 
$b(\phi \eta), b(\omega \eta), b\left(\rho^{0} \eta\right), b\left(\rho^{0} \pi^{0}\right), b\left(\omega \pi^{0}\right)$, and $b\left(\phi \pi^{0}\right)$.

$$
\begin{aligned}
a\left(\phi K^{0}\right)= & \frac{1}{2}\left[b\left(\bar{K}^{* 0} K^{0}\right)-b\left(K^{* 0} \bar{K}^{0}\right)\right]+\frac{1}{2} \sqrt{\frac{3}{2}}\left[c b(\phi \eta)-s b\left(\phi \eta^{\prime}\right)\right] \\
& +\frac{\sqrt{3}}{4}\left[c b(\omega \eta)-s b\left(\omega \eta^{\prime}\right)\right]-\frac{\sqrt{3}}{4}\left[c b\left(\rho^{0} \eta\right)-s b\left(\rho^{0} \eta^{\prime}\right)\right] \\
& +\frac{1}{4} b\left(\rho^{0} \pi^{0}\right)-\frac{1}{4} b\left(\omega \pi^{0}\right)-\frac{1}{2 \sqrt{2}} b\left(\phi \pi^{0}\right)
\end{aligned}
$$

Where the quantities $c$ and $s$ are given by $c=\cos \theta_{\eta \eta^{\prime}}$ and $s=\sin \theta_{\eta \eta^{\prime}}$, and $\theta_{\eta \eta^{\prime}}=20^{\circ}$ is the $\eta-\eta^{\prime}$ mixing angle [9].

Equation (1.35) for $\Delta S_{\phi K^{0}}$ can not be immediately applied as the $\mathrm{SU}(3)$ relations do not provide an upper bound on $\left|\xi_{\phi K}\right|$, but rather on $\hat{\xi}_{\phi K}$ defined by

$$
\hat{\xi}_{\phi K} \equiv \frac{V_{u s}}{V_{u d}} \times \frac{V_{c b}^{*} V_{c d} a_{\phi K}^{c}+V_{u b}^{*} V_{u d} a_{\phi K}^{u}}{V_{c b}^{*} V_{c s} a_{\phi K}^{c}+V_{u b}^{*} V_{u s} a_{\phi K}^{u}}
$$

The SU(3) relations shown in Eq. (1.36) yield the following constraint on $\left|\hat{\xi}_{\phi K}\right|$

$$
\begin{aligned}
\left|\hat{\xi}_{\phi K}\right| & <\frac{1}{\sqrt{\mathcal{B}\left(\phi K_{S}^{0}\right)}} \frac{\left|V_{u s}\right|}{\left|V_{u d}\right|}\left\{\frac{1}{2}\left[\sqrt{\mathcal{B}\left(\overline{\left.K^{* 0} K^{0}\right)}\right.}+\sqrt{\mathcal{B}\left(K^{* 0} \bar{K}^{0}\right)}\right]\right. \\
& +\frac{1}{2} \sqrt{\frac{3}{2}}\left[c \sqrt{\mathcal{B}(\phi \eta)}+s \sqrt{\mathcal{B}\left(\phi \eta^{\prime}\right)}\right]+\frac{\sqrt{3}}{4}\left[c \sqrt{\mathcal{B}(\omega \eta)}+s \sqrt{\mathcal{B}\left(\omega \eta^{\prime}\right)}\right] \\
& +\frac{\sqrt{3}}{4}\left[c \sqrt{\mathcal{B}\left(\rho^{0} \eta\right)}+s \sqrt{\mathcal{B}\left(\rho^{0} \eta^{\prime}\right)}\right]+\frac{1}{4} \sqrt{\mathcal{B}\left(\rho \pi^{0}\right)} \\
& \left.+\frac{1}{4} \sqrt{\mathcal{B}\left(\omega \pi^{0}\right)}+\frac{1}{2 \sqrt{2}} \sqrt{\mathcal{B}\left(\phi \pi^{0}\right)}\right\}
\end{aligned}
$$

where the branching ratios in the numerator of Eq. (1.38) can be either measurements 
or limits. The minus signs between the branching ratios in Eq. (1.36) have been replaced with plus signs due to the fact that this is an upper bound on $\left|\hat{\xi}_{\phi K}\right|$ and the relative phases between the amplitudes are all assumed to be maximal (ie. $\pi$ ).

$\hat{\xi}_{\phi K}$ is related to $\left|\xi_{\phi K}\right|$ in Eq. (1.35) for $\Delta S_{\phi K^{0}}$ by the following [18]

$$
\left|\hat{\xi}_{\phi K}\right|^{2}=\frac{\left|\frac{V_{u s} V_{c d}}{V_{c s} V_{u d}}\right|^{2}+\left|\xi_{\phi K}\right|^{2}+2 \cos \gamma \mathcal{R} e\left(\frac{V_{u s} V_{c d}}{V_{c s} V_{u d}} \xi_{\phi K}\right)}{1+\left|\xi_{\phi K}\right|^{2}+2 \cos \gamma \mathcal{R} e\left(\xi_{\phi K}\right)} .
$$

This equation can be solved numerically for $\left|\xi_{\phi K}\right|$ in order to calculate $\Delta S_{\phi K^{0}}$ using Eq. (1.35).

Eleven branching ratios are required to calculate $\left|\xi_{\phi K}\right|$ and none of these modes have been observed, although there is evidence for $B^{0} \rightarrow \rho^{0} \pi^{0}$. Upper limits have been placed on all the necessary branching ratios except the $B^{0} \rightarrow \bar{K}^{* 0} K^{0}$ and $K^{* 0}$ $\bar{K}^{0}$ channels. This analysis, therefore, will allow an upper limit on $\Delta S_{\phi K^{0}}$ to be calculated for the first time using the SU(3) symmetry technique. The current combined contribution to $\Delta S_{\phi K^{0}}$ from the limits on the other channels is $\left|\Delta S_{\phi K^{0}}\right|<0.32$. $\mathrm{SU}(3)$ flavor symmetry is expected to be broken at roughly the $30 \%$ level, however this calculation assumes no $\mathrm{SU}(3)$ flavor symmetry breaking.

\subsection{The $\sin 2 \beta$ Discrepancy $\left(\Delta S_{\phi K^{0}}\right)$}

A principal motivation for this study is to help clarify issues concerning a possible discrepancy between $\sin 2 \beta$ results obtained using tree- and penguin-dominated $B^{0}$ 
decays. In particular,this analysis is relevant for the interpretation of the $\sin 2 \beta$ result obtained from the $B^{0} \rightarrow \phi K^{0}$ channel as described in the preceding sections.

The $\sin 2 \beta$ result from the $\phi K^{0}$ channel is expected to be accurate to within a few per cent [9]. However, BABAR and BELLE independently observe the $\sin 2 \beta$ result from $B^{0} \rightarrow \phi K^{0}$ to be about $30 \%$ smaller than the corresponding result from the treedominated $b \rightarrow c$ decays [13]. While this difference is not statistically significant, a similar trend is observed between the tree-level results and those of other penguindominated processes [13]. Figure 1.8 shows the current status of measurements of $\sin 2 \beta$ using penguin-dominated modes.

The observed differences may be due to statistical fluctuations, SM pollution, or new physics beyond the SM. Using the $\mathrm{SU}(3)$ relations described in the preceding section, the results from this analysis, in conjunction with the other branching ratio limits, will allow an upper bound to be calculated on $\Delta S_{\phi K^{0}}$ due to SM pollution for the first time using the $\mathrm{SU}(3)$ symmetry technique.

It is worth noting that when we began this analysis in 2004, the observed differences in Fig. 1.8 were generally larger. At that time the difference between the measurement of $\sin 2 \beta$ using the charmonium modes and the naïve average of the $b \rightarrow s$ penguin modes was $3.7 \sigma$. New results within the past year have reduced this difference to approximately $2.7 \sigma$. 


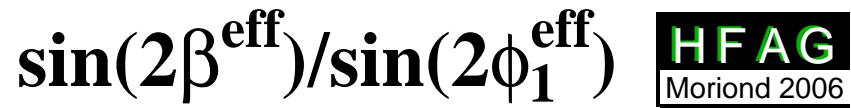 \\ PRELIMINARY}

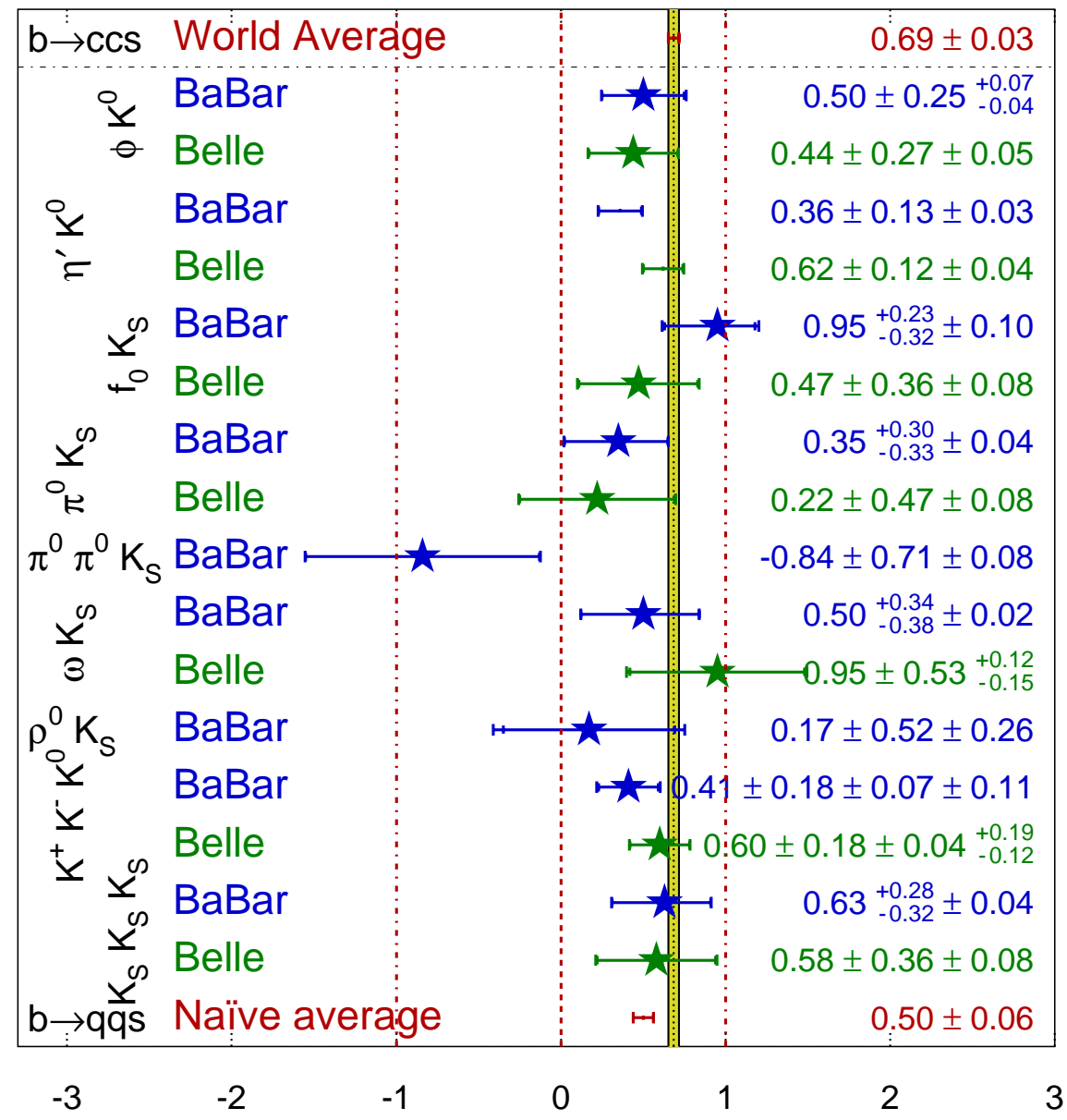

Figure 1.8: Current status of $\sin 2 \beta$ from penguin-dominated modes. 


\section{Chapter 2}

\section{PEP-II and the BABAR Detector}

\subsection{The LINAC Accelerator and PEP-II Storage Ring at SLAC}

PEP-II is an asymmetric $e^{+} e^{-}$storage ring where the electrons are accelerated to an energy of $8.985 \mathrm{GeV}$ and the positrons to an energy of $3.112 \mathrm{GeV}$. The two beams produce a center-of-mass energy of $10.58 \mathrm{GeV}$ to match the Upsilon $4 \mathrm{~S}$ resonance. Electrons and positrons are first generated and accelerated using the linear accelerator (LINAC) at SLAC. Electrons are generated by electrically heating a filament and then accelerated into copper waveguides by an electric field. After being accelerated to an energy of approximately $10 \mathrm{MeV}$, the beam is directed into a damping ring to reduce the transverse momentum of the electrons in the beam. The beam is then directed back into the LINAC where Klystrons generate high power microwaves in 
radio frequency (rf) cavities that accelerate the electrons to an energy of $9.0 \mathrm{GeV}$.

To generate positrons a portion of the electron beam is separated and collided with a titanium target to generate electron positron pairs. The positrons are then returned to the LINAC to be accelerated and damped as described above for the electron beam. After damping the positrons are accelerated to an energy of $3.1 \mathrm{GeV}$.

Once accelerated to the desired energy, electrons and positrons are injected into the PEP-II storage rings on a semi-continuous basis to maintain the desired beam currents. The electrons are injected into the High Energy Ring (HER) and travel clock-wise around the ring while the positrons are injected into the Low Energy Ring (LER) and travel counter clock-wise around the ring. Magnets and rf cavities around the storage ring maintain the focus and replace energy lost due to synchrotron radiation and bring the two beams into collision at one interaction point (IP). The BABAR detector is constructed around the IP to detect and measure the decay products of the resulting $e^{+} e^{-}$collisions. Figure 2.1 shows an overview of the LINAC, PEP-II, and the BABAR detector.

\subsubsection{Design and Performance}

The accelerator and storage ring were designed to operate at an instantaneous luminosity of $3.00 \times 10^{33} \mathrm{~cm}^{-2} \mathrm{~s}^{-1}$ and provide an integrated luminosity of $3.3 \mathrm{fb}^{-1}$ per month on a continuous or "factory" basis. The accelerator system has significantly exceeded these original objectives. On October 9, 2005 the system achieved a record 


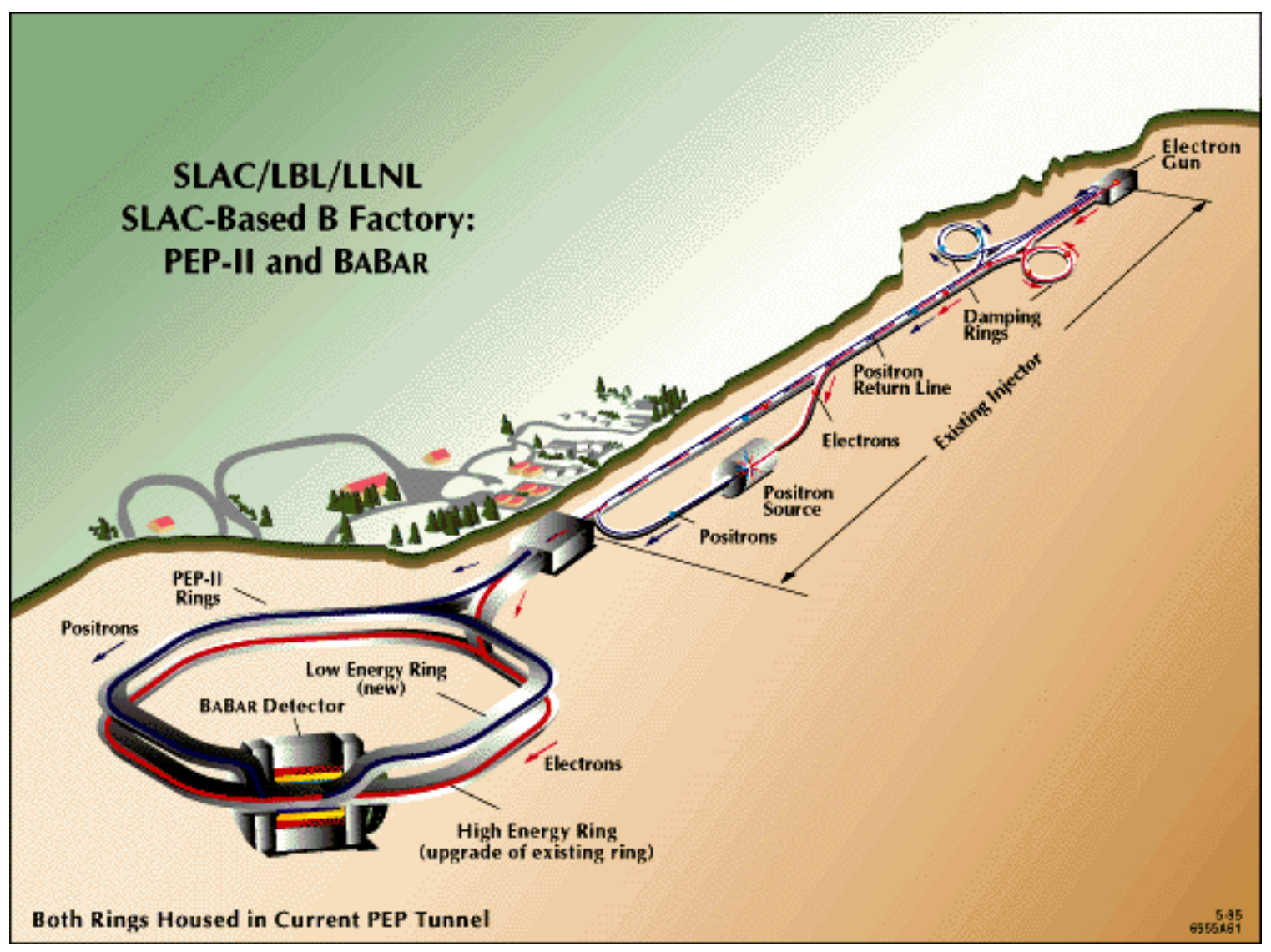

Figure 2.1: Overview of the LINAC, PEP-II, and the BABAR detector at SLAC. 
instantaneous luminosity of $10.025 \times 10^{33} \mathrm{~cm}^{-2} \mathrm{~s}^{-1}$ or more than 3 times the design luminosity, and has delivered a total of $341 \mathrm{fb}^{-1}$ to the BABAR detector during the past 81.6 months. Figure 2.2 shows the integrated luminosity delivered since operations first began in June 1999. Table 2.1 shows several parameters used to measure the accelerator systems performance and includes the design value and the value as of May 2005 for each parameter [19].

The luminosity the accelerator system is able to deliver is based on several factors that are included in the luminosity scaling equation

$$
L=2.17 \times 10^{34}(1+r) \xi_{y}\left(\frac{E I}{\beta_{y}^{*}}\right) \mathrm{cm}^{-2} \sec ^{-1}
$$

where $r$ is the $\mathrm{y}$ to $\mathrm{x}$ aspect ratio $(\sim 0.04), E$ is the beam energy, $I$ is the beam current, $\xi_{y}$ is the vertical tune shift limit (related to the mutual focusing of the two beams), and $\beta_{y}^{*}$ the vertical beta at the collision point (related to the size of the beam).

Table 2.1: Accelerator system performance parameters.

\begin{tabular}{|l|l|l|}
\hline Parameter & Design Value & Present Value \\
\hline HER Current $(\mathrm{mA})$ & 750 & 1745 \\
LER Current $(\mathrm{mA})$ & 2140 & 2995 \\
HER RF volts $(\mathrm{MV})$ & 14.0 & 15.5 \\
LER RF volts (MV) & 3.4 & 4.04 \\
$\beta_{y}^{*}(\mathrm{~mm})$ & $15-25$ & 11 \\
HER Vertical $\xi_{y}$ & 0.03 & 0.046 \\
LER Vertical $\xi_{y}$ & 0.03 & 0.064 \\
Peak Int. Lumi/month $\left(\mathrm{fb}^{-1}\right)$ & 3.3 & 17.04 \\
Total Int. Lumi $\left(\mathrm{fb}^{-1}\right)$ & $30 /$ year & 341.3 total \\
\hline
\end{tabular}


03/20/2006 04:22

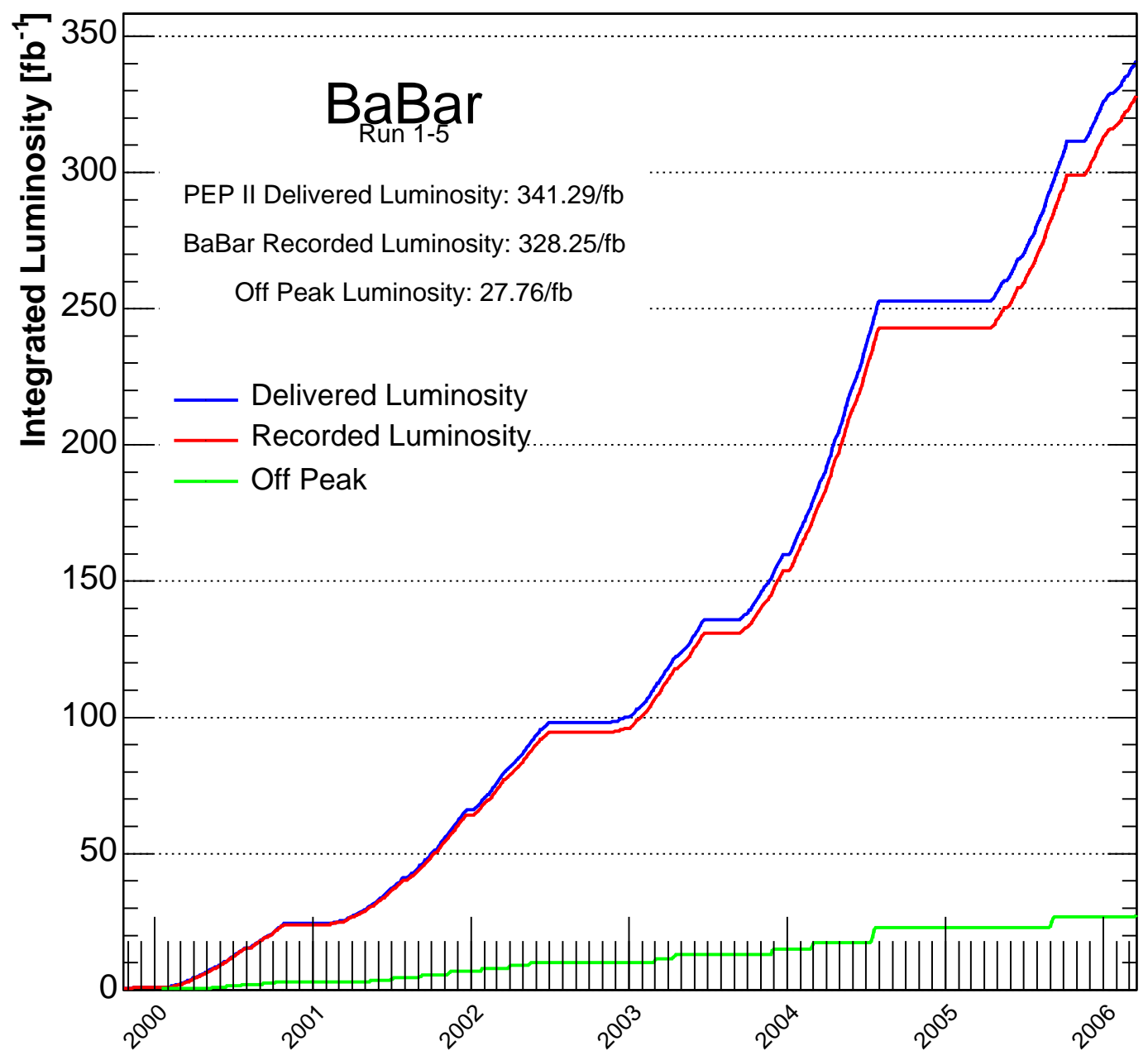

Figure 2.2: Integrated luminosity from June 1999 through March 20, 2006. 


\subsubsection{Background Radiation and the Machine-Detector In- terface (MDI)}

As beam currents and luminosity have increased the amount of background radiation affecting the detector has increased. High doses of radiation can damage the detector and degrade subsystem performance. In addition, excessive background events can saturate the data acquisition system and lead to excessive dead time where the detector is unable to accept new data because the system is full. To protect the detector the SVTrad system (see Sect. 2.3) monitors the radiation levels at several points around the SVT and will abort the beams whenever it exceeds certain instantaneous and integrated limits.

Background radiation can occur due to beam instabilities, scattering from particles in the beam pipe (so called "dust" events or out-gassing from components within the beam pipe), and scattering from collimators and other components within the beam pipe. Extensive simulation studies have lead to modifications of the component geometry within various sections of the beam pipe to reduce sources of background radiation. Additional shielding has also been installed to protect the forward end-cap of the IFR (Sect. 2.7).

Electrons and positrons are injected into the storage ring in small amounts on a regular basis with the detector in operation (called trickle injection). During trickle injection the beams are not suitable for data taking for the first few revolutions around the ring due to high background levels. To reduce the impact on the data 
acquisition system, an inhibit window was implemented which blocks event triggers for approximately $10 \mathrm{~ms}$ after each injection pulse.

\subsection{Overview of the BABAR Detector}

The BABAR detector consists of five major subsystems: a silicon vertex tracker (SVT), a drift chamber (DCH), a ring-imaging Cherenkov detector (DIRC), a CsI calorimeter, and a muon detector (IFR). A superconducting solenoid provides a 1.5T magnetic field to allow momentum measurements in the DCH. This field would adversely affect the operation of the photo-multiplier tubes (PMTs) within the the DIRC; consequently a "bucking coil" is used to counteract the magnetic field near the PMT portion the DIRC. Figures 2.3 and 2.4 show schematic drawings of the side and front views of the detector. The polar angle coverage of the detector is $137^{\circ}$ which extends from $20.1^{\circ}$ (350 $\mathrm{mrad}$ ) from the beam line in the forward (HER) direction to $22.9^{\circ}$ (400 mrad) from the beam line in the backward (LER) direction. The IP is offset from the center of the detector by $370 \mathrm{~mm}$ in the backward direction to increase coverage in the boosted direction.

The SVT and the DCH make up the primary charged particle tracking system. The momentum of charged particles is determined in the DCH by measuring the curvature of tracks in the magnetic field created by the solenoid. The DCH also provides a primary input for the trigger and provides a measurement of ionization energy loss $(d E / d x)$ for particle identification. The DIRC provides the primary means 


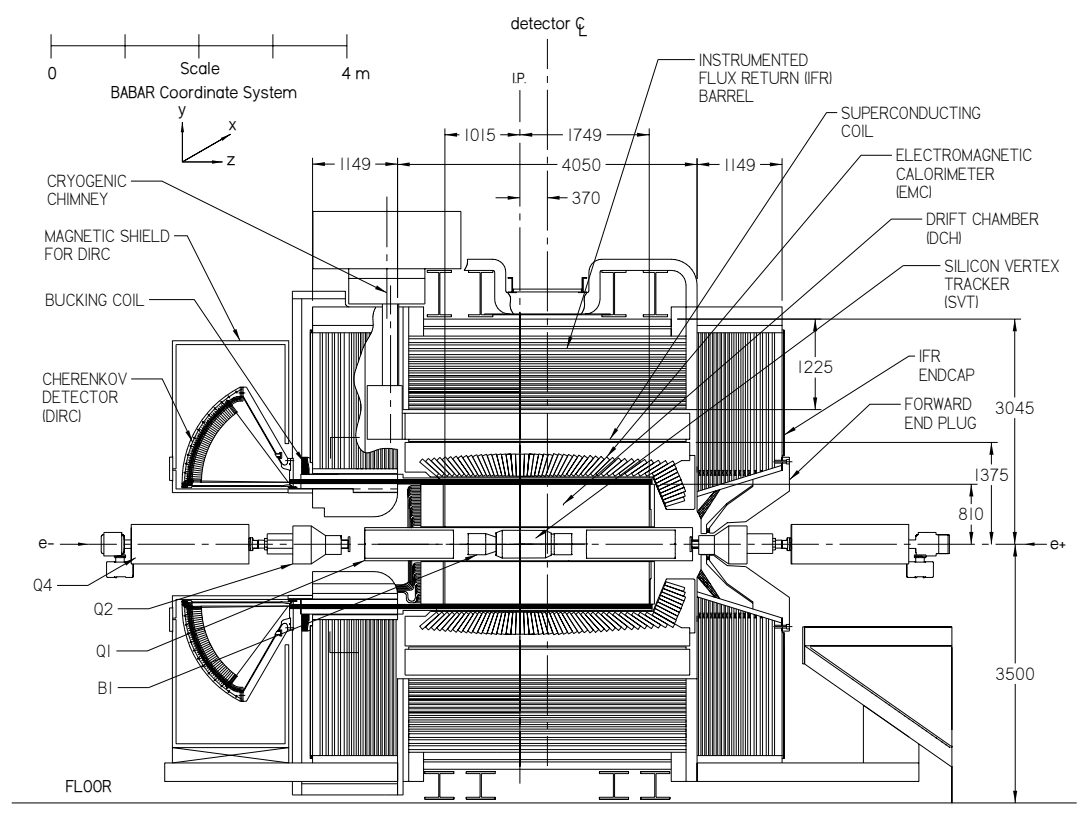

Figure 2.3: Longitudinal section of the BABAR detector. The dimensions shown are in millimeters.

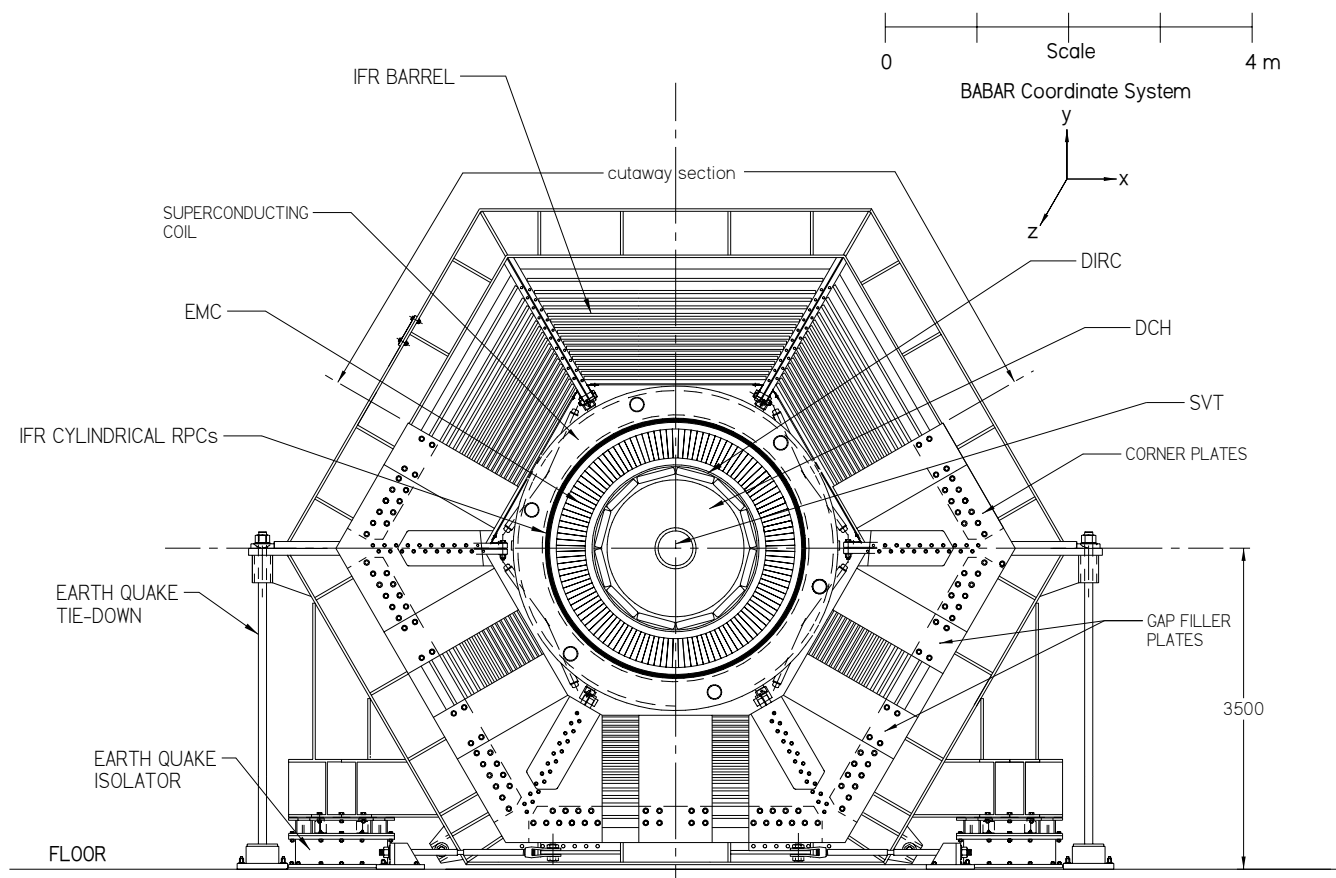

Figure 2.4: End view of the BABAR detector. 
for separating pions from kaons. The EMC detects electromagnetic showers over the energy range from $20 \mathrm{MeV}$ to $4 \mathrm{GeV}$ and is used to detect and measure the energy of neutral particles and photons. The IFR is designed to detect muons and neutral hadrons. The trigger system uses information from the DCH, EMC, and IFR to determine if the data from a particular event is to be saved for later analysis. For all events selected by the trigger, the DAQ collects and stores all the information from each component, reconstructs physics events, and stores the data for later off-line analysis. Each of the major subsystems is described in more detail in the following sections. For a complete description of the BABAR detector see [21].

\subsection{The Silicon Vertex Tracker (SVT)}

\subsubsection{SVT Theory of Operation}

The SVT operates as a reverse-biased p-n junction diode. The "p" type semiconductor is doped with acceptor atoms (atoms with one less valence electron) and "n" type semi-conductors are doped with donor atoms (atoms with one additional

electron in the outer shell). When the two materials are brought into contact and reverse-biased (positive on the $\mathrm{n}$-side and negative on the p-side) the electric field pulls the electrons and holes apart and creates a depletion region between them. In a detector this depletion region is widened by placing undoped silicon between the $\mathrm{p}$ and $\mathrm{n}$ doped material. 
In the absence of charged particles there is a small current due to thermal generation of charge carriers. When a high energy charged particle traverses the depletion region it creates a uniform density of electron-hole pairs along its path. The bias voltage attracts the electrons and holes in opposite directions creating a current. The signal is then read out as a change in the bias current.

Silicon is used due to it's relatively low ionization energy (3.6 eV), long mean free path for electrons $(\sim 100 \mathrm{~nm})$, and large energy loss $(d E / d x)$ for minimum ionizing particles $(3.8 \mathrm{MeV} / \mathrm{cm})$. This combination provides many charge carriers per event, a high charge collection efficiency, and large signals. For additional details regarding the silicon detector theory see, for example [24].

\subsubsection{SVT Design}

The SVT (Figure 2.5) is composed of five layers of double-sided silicon strips. The strips on the opposite sides of each layer are oriented at right angles with the $\phi$ strips parallel to the beam axis and the $z$ strips transverse. The inner three layers are flat while the outer two layers are arched to reduce the amount required to cover the full circumference. Stiffness for the assembly is provided by carbon fiber/Kevlar ribs (see fig. 2.5).

The $z$ strips vary in length from $40 \mathrm{~mm}$ in layer 1 to $104 \mathrm{~mm}$ in layer 5 . For the inner layers 1, 2 and 3 each $z$ strip is individually connected to the electronics. For layers 4 and 5 two strips are connected together and readout as one. The $\phi$ strips range from 
$82 \mathrm{~mm}$ in length in layer 1 to $265 \mathrm{~mm}$ in layer 5 and are all individually connected to the electronics. The signal is read out by approximately 150,000 separate channels. To minimize the amount of material in the acceptance region the readout electronics are mounted outside the detector area. Figures 2.6 and 2.7 show schematic views of the SVT.

Alignment of the SVT is divided into two separate activities: the local or internal alignment of each layer relative to the other layers and the global or external alignment of the SVT as a whole relative to the rest of the BABAR detector. Both alignments are critical to maintain precise vertexing and tracking information. The internal alignment of the SVT is relatively stable and is generally only performed after the magnet has been shut down for some reason or work has been performed on the detector. The external alignment can vary on an hourly basis and shows a diurnal pattern typical of daily temperature fluctuations. The diurnal variation in position is typically $\pm 50 \mu \mathrm{m}$. The external alignment is therefore updated for each run or about once each hour. The internal alignment procedure uses $e^{+} e^{-} \rightarrow \mu^{+} \mu^{-}$ events and cosmic rays. The external alignment procedure treats the SVT as a rigid body and compares track positions as measured by the SVT with those measured by the DCH. This data is collected during each run and used to update the alignment for the following run. 


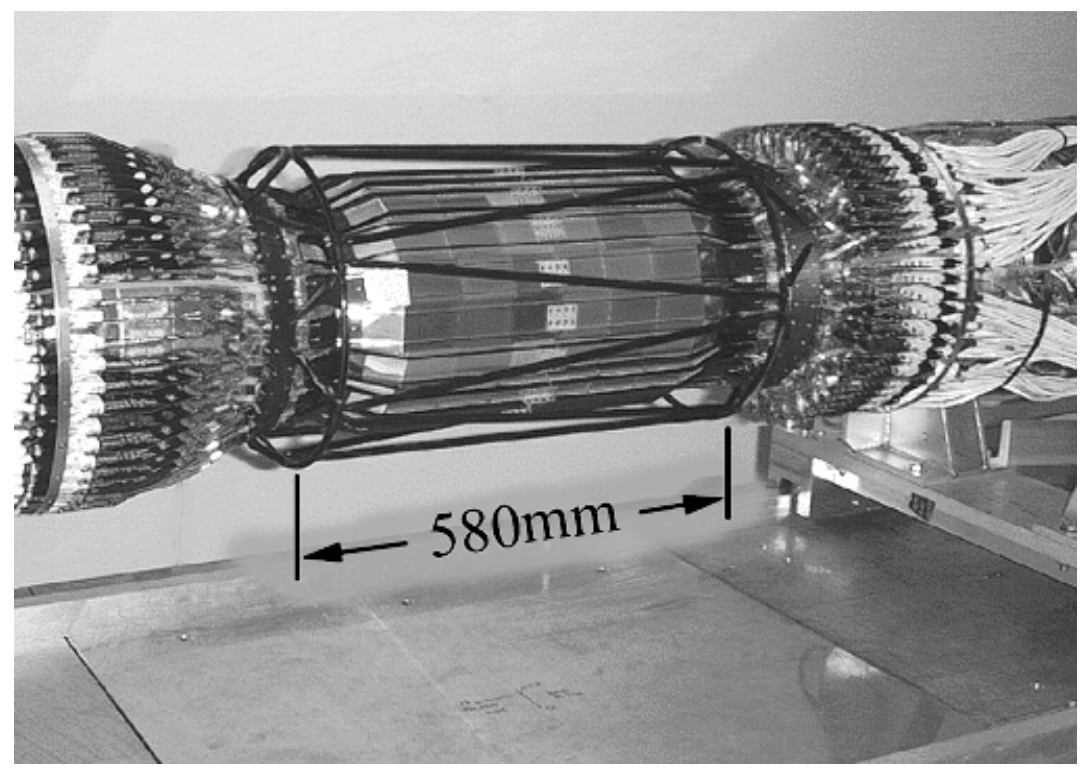

Figure 2.5: Photo of the completed SVT.

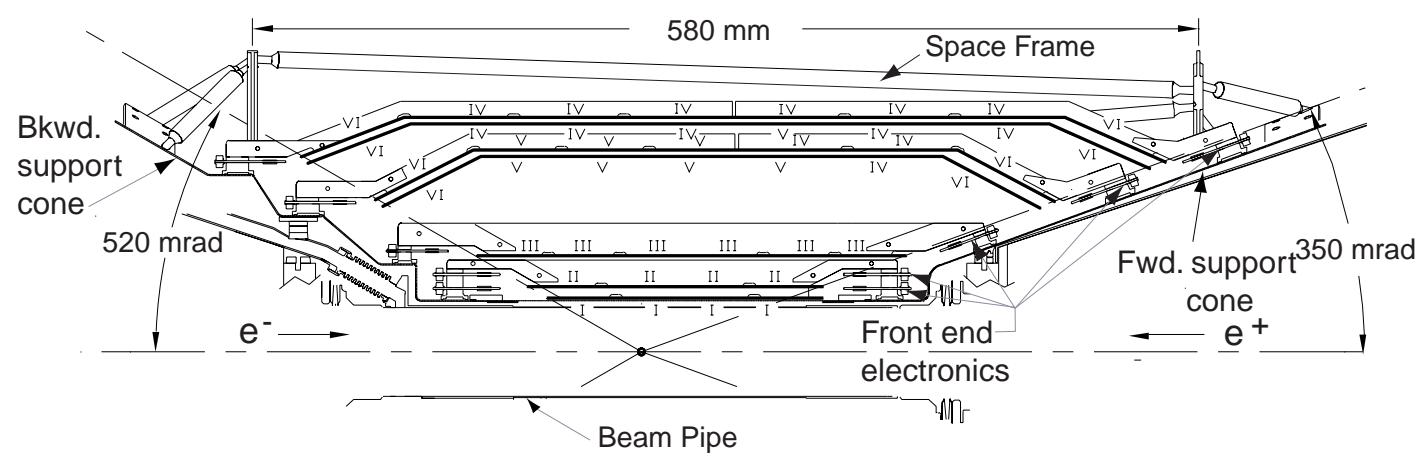

Figure 2.6: Longitudinal schematic drawing of the SVT. 


\subsubsection{SVT Radiation Monitoring and Protection System (SV- TRAD)}

The SVTRAD system is designed to monitor the radiation dose being absorbed by the BABAR detector and to abort the beams if necessary to protect the detector from potentially damaging radiation doses. The system consists of $12 \mathrm{p}$-i-n junction diodes (PIN diodes) and 2 polycrystalline chemical vapor deposition (CVD) diamonds placed at both ends of the SVT. The PIN diodes are $10 \mathrm{~cm}$ from the IP and the diamonds are $15 \mathrm{~cm}$. The performance of the PIN diodes has degraded since initial installation and they being replaced by the diamonds. The abort system checks for both short spikes in radiation as well as the integrated dose and is hardwired to abort the beams when certain thresholds are exceeded. Elevated radiation doses that have not yet reached abort levels start an abort timer and notifies both the PEP-II operator and the BABAR Shift Leader that the beams will be aborted unless radiation levels are reduced within five minutes. The number of beam aborts varies widely depending on beam conditions and ranges from less than one per day to more than 10 per day. Figure 2.8 shows the integrated radiation dose at four locations around the SVT.

\subsubsection{SVT Performance}

SVT performance is measured by the probability that a hit in the SVT matches a track in the DCH. It is therefore a combination of hardware hit efficiency and software reconstruction efficiency. The most recent tests of the efficiency of the SVT indicate 


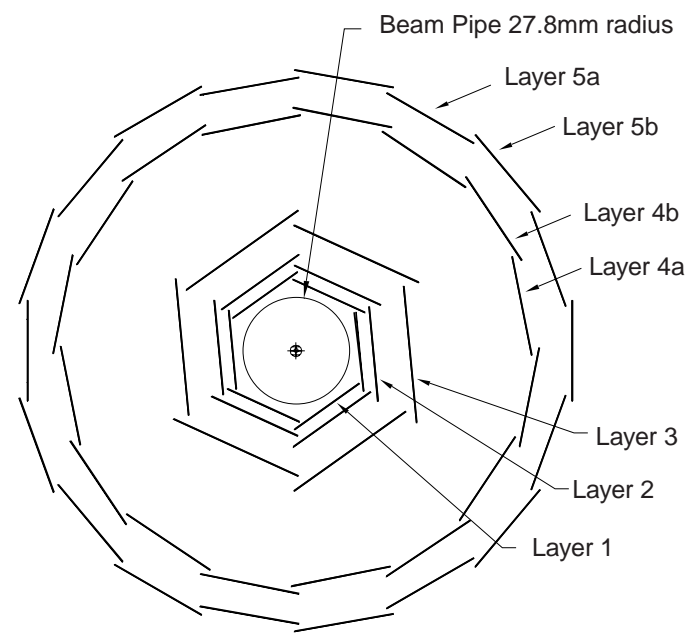

Figure 2.7: End view of the SVT showing the five layers.

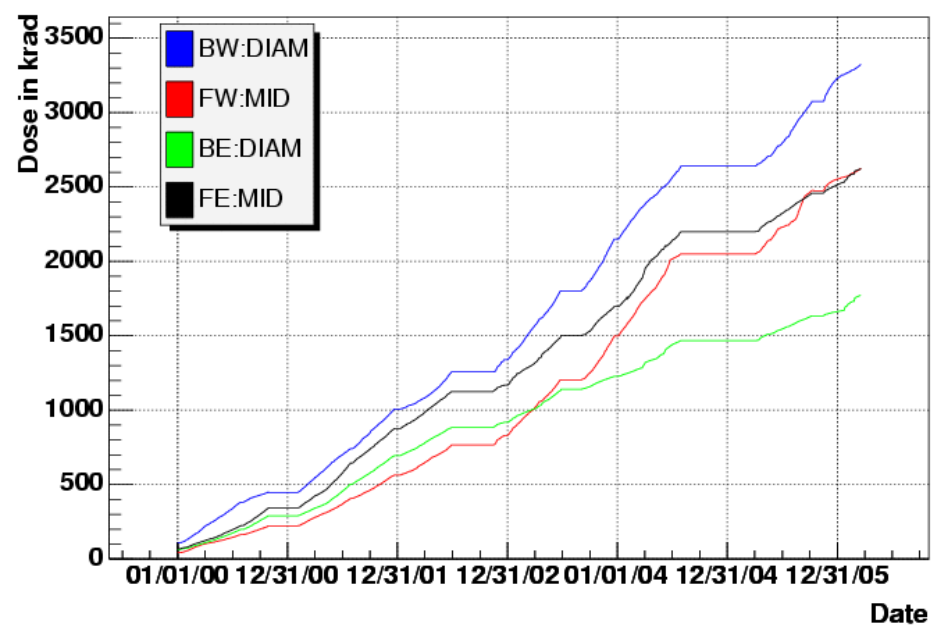

Figure 2.8: Integrated radiation dose at four locations around the SVT. 
that all layers are roughly $98 \%$ efficient. Another measure of performance is the hit resolution measured in micrometers. Figures 2.9 and 2.10 show the $z$ and $\phi$ resolution for each layer of the SVT. 


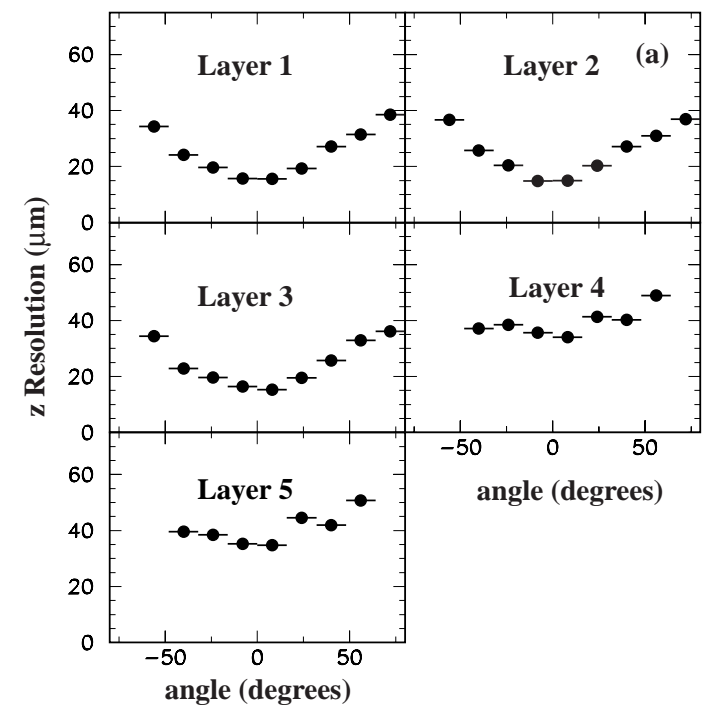

Figure 2.9: Z resolution of the SVT in micrometers.

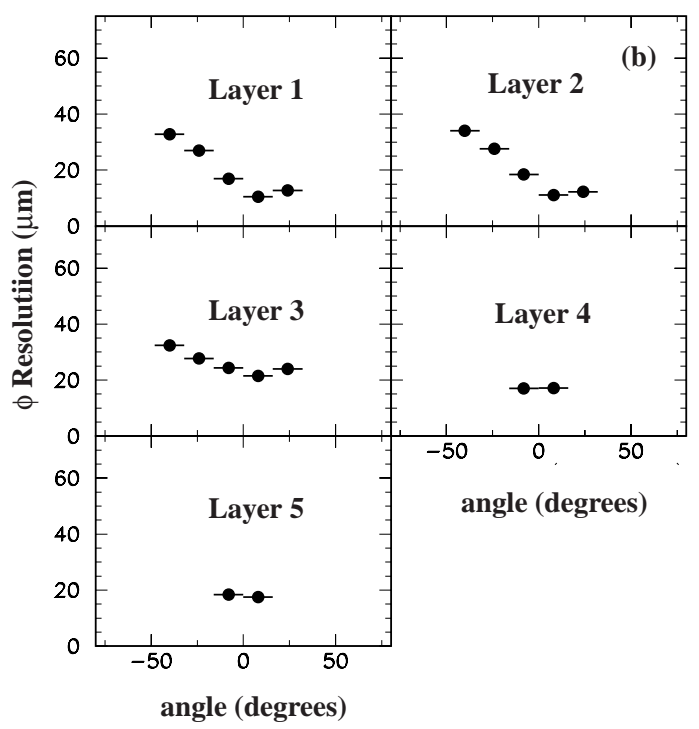

Figure 2.10: SVT $\phi$ resolution in micrometers. 


\subsection{The Drift Chamber (DCH)}

\subsubsection{DCH Theory of Operation}

The Drift Chamber consists of a gas filled chamber traversed by a series of wires. A charged particle with sufficient energy passing through the chamber will ionize the gas along its path of travel. If an electric potential is maintained between the wires, the ionized electrons are accelerated toward the positively charged (sense) wires and away from the negatively charged (field) wires. The accelerating electrons cause an avalanche of secondary ionization which amplifies the original signal and produces a pulse in the current on the sense wire and defines a "hit". The path is determined by the arrival times of the pulses and the pattern of hits.

The critical components are the field and sense wires along with the gas mixture. The wires must be kept as small as possible to maximize the electric field at the surface of the sense wires for a given value of potential. A high field region near the sense wires is critical to charge multiplication. The gas mixture is a trade-off between ionization energy and radiation length which is defined as the distance a particle travels while its energy falls to $1 / e$ of its initial value. A low ionization energy is desirable to generate large numbers of electrons with a minimum reduction in the energy of the particle passing through the gas. A long radiation length is desirable to minimize the reduction in the particles energy in passing through the chamber. Nobel gases such as Argon have relatively low ionization energies (15.8 
$\mathrm{eV}$ for Argon) and moderate radiation lengths (14cm for Argon). Whereas Helium has a somewhat higher ionization energy $(24.6 \mathrm{eV})$ but much longer radiation length $(756 \mathrm{~cm})$.

A critical problem is the potential breakdown of the chamber due to the photoelectric effect. Excited atoms within the chamber will emit photons with sufficient energy to eject electrons from the field wires. These electrons will then start a new avalanche and disrupt the operation of the chamber. To avoid this occurrence a quenching gas is used which is capable of absorbing and thermalizing a wide range of photon energies.

An additional concern for drift chambers is the Malter effect [26]. As a drift chamber ages, it can develop current spikes that damage the wires. The cause is believed to be the Malter effect where a dielectric polymer builds up on the field wires. This leads to an accumulation of positive charges near the surface of the field wire and causes electron emission and a resulting avalanche. Water vapor added to the gas mixture appears to prevent the Malter effect [25].

The trajectory of a charged particle in a uniform magnetic field will be a helix. The radius of curvature, $R$ in meters, and the pitch angle, $\alpha$ of this helix are related to the component of the momentum transverse to the magnetic field, $p_{t}$, and the strength of the magnetic field, $B$ in Telsa by [9], sec. 28.12 .

$$
p_{t} \cos \alpha=0.3 z B R
$$


where ze is the charge of the particle. Drift chambers are therefore generally placed in uniform magnetic fields parallel to the beam direction to allow measurement of the transverse momentum.

In addition to providing momentum measurements, the DCH is also used for particle identification by measuring $d E / d x$, the energy lost to ionization per unit path length or specific ionization. The total charge collected by a sense wire for a given track is proportional to the $d E / d x$ for that track. $d E / d x$ in turn is related to the particle velocity $(\beta \gamma)$ using the Bethe-Bloch formula

$$
-\frac{d E}{d x}=K z^{2} \frac{Z}{A} \frac{1}{\beta^{2}}\left[\frac{1}{2} \ln \frac{2 m^{e} c^{2} \beta^{2} \gamma^{2} T_{\max }}{I^{2}}-\beta^{2}-\frac{\delta}{2}\right]
$$

where $\mathrm{K}=0.307 \mathrm{MeV} g^{-1} \mathrm{~cm}^{2}, z$ is the charge of the incident particle, $Z$ and $A$ are the atomic number and atomic mass respectively of the absorber, $m_{e}$ is the electron mass, $T_{\max }$ is the maximum kinetic energy which can be imparted to a free electron in a single collision, $I$ is the mean excitation energy in $\mathrm{eV}, \delta$ is a density effect correction to the ionization energy loss, and $c, \beta$, and $\gamma$ have their usual definitions in relativistic kinematics. Once the momentum and velocity are known the mass can be easily calculated.

In the BABAR detector the DIRC (Sect. 2.5) is the primary means of particle identification. However, for low momentum particles and particles in the extreme forward and backward directions, $d E / d x$ using the DCH and SVT is the only means for PID. 


\subsubsection{DCH Design}

The BABAR drift chamber is composed of 28,768 wires in 7,104 hexagonally shaped drift cells, and arranged in 40 layers. Sequential layers are staggered by half a cell. The wires in 24 of the 40 layers are at small angles with respect to the $z$ axis to provide longitudinal position information. The inner support cylinder is made from beryllium and aluminum and the outer cylinder is carbon fiber composite. The center of the drift chamber is offset $370 \mathrm{~mm}$ from the IP in the backward direction. Figure 2.11 shows a schematic of the BABAR drift chamber.

The sense wires are $20 \mu \mathrm{m}$ in diameter and made of gold plated tungsten-rhenium. The field wires are gold plated aluminum $120 \mu \mathrm{m}$ in diameter. The sense wires and field wires are currently maintained at a potential difference of 1930 volts. The drift cells are made up of one sense wire surrounded by six field wires in a hexagonal shape. Figure 2.12 shows the layout of the four innermost layers.

The drift chamber uses a gas mixture of $80 \%$ helium and $20 \%$ isobutane as the quenching gas. This mixture has a radiation length of $807 \mathrm{~m}$ and is designed to minimize multiple scattering inside the DCH. 3500 ppm of water vapor is added to the gas to prevent the Malter effect.

\subsubsection{DCH Performance}

Data from the DCH is used for tracking, PID, and momentum determination. Track reconstruction efficiency has been measured over a range of transverse momenta and 
polar angles. Figure 2.13 shows the measured tracking efficiencies for two different operating voltages (the $\mathrm{DCH}$ is currently operating between these two voltages at 1930). The efficiency is measured based on multi-hadron events as the fraction of tracks detected in the SVT that are also detected in the DCH. The track reconstruction efficiency in the DCH is greater than $95 \% . d E / d x$ measurement resolution in the DCH is shown in Figure 2.14 and has a resolution of $7.5 \%$ based on a DCH operating voltage of 1900 volts. 


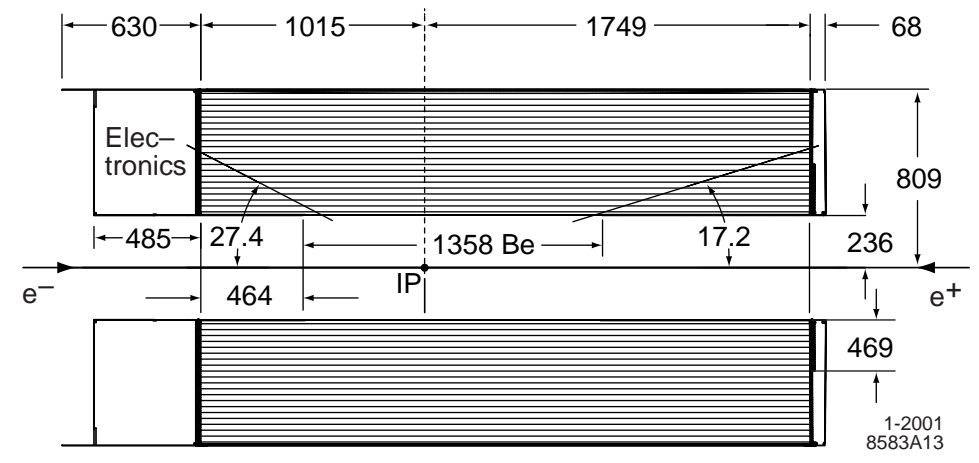

Figure 2.11: Longitudinal section of the BABAR drift chamber. The dimensions are in millimeters. 


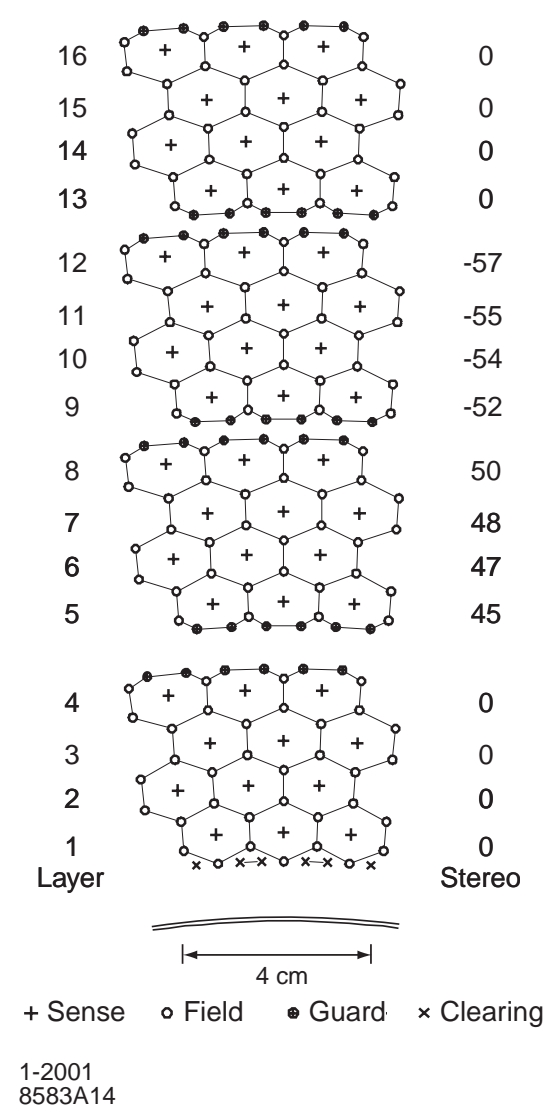

Figure 2.12: Layout of the four innermost layers of the DCH. Lines have been added between the field wires as a visual aide. The "Stereo" numbers on the right side indicate the offset angle (mrad) of the sense wires in that layer for $\mathrm{z}$ position measurements. 

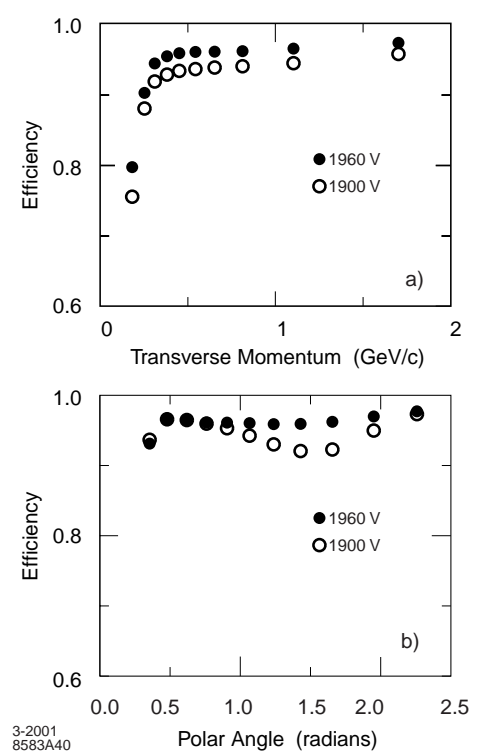

Figure 2.13: Track reconstruction efficiency for operating voltages of 1900 and 1960 Volts. Tracking efficiency is shown versus the transverse momentum in plot "a" and versus the polar angle in plot "b".

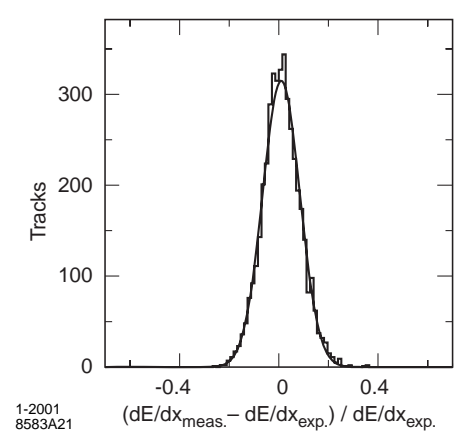

Figure 2.14: The histogram shows the difference between the measured and expected energy loss, $d E / d x$ from Bhabha scattering. The curve is a fitted Gaussian with a resolution of $7.5 \%$. 


\subsection{The Detector of Internally Reflected Cherenkov light (DIRC)}

\subsubsection{DIRC Theory of Operation}

The DIRC is fundamentally a ring-imaging Cherenkov detector which uses total internal reflection to transfer the Cherenkov radiation out of the detector to externally mounted photo-multiplier tubes (PMTs). Particles traversing a medium at a velocity greater than the speed of light in that medium emit Cherenkov radiation at an angle $\theta_{c}$ relative to the particles direction. This angle is related to the particle's velocity

( $\beta)$ by $\cos \theta_{c}=1 / n \beta$, where $n$ is the index of refraction of the medium. In the DIRC the material is fused silica with $n=1.473$. The angle $\phi_{c}$ denotes the azimuthal angle of the Cherenkov photon around the particle's direction.

Since all particles of interest have $\beta \approx 1$, some photons will always lie within the total internal reflection limit of the silica bars and will be reflected to one end of the bar or the other. The process of total internal reflection preserves the initial angles of the photons. Cherenkov photons generated in the bar are focused onto the phototubes. Since the track trajectory is known from the SVT and DCH, the Cherenkov angles $\theta_{c}$ and $\phi_{c}$ can be determined from the geometry of the PMT distribution. Figures 2.16 shows a typical optical path of a photon in the DIRC. 


\subsubsection{DIRC Design}

The DIRC consists of 144 fused silica bars $17 \mathrm{~mm}$ thick, $35 \mathrm{~mm}$ wide, and $4.9 \mathrm{~m}$ long held in an aluminum tube. 10,752 PMTs are housed in a Standoff Box at the backward end of the detector containing 6,000 liters of highly purified water. A "bucking coil" surrounds the standoff box to reduce the magnetic field in the PMT region to less than 1 Gauss. Filtered nitrogen gas is circulated through each bar box to prevent moisture from condensing on the bars. Figure 2.15 shows the geometry and overall layout of the DIRC.

\subsubsection{DIRC Performance}

A control sample of $D^{0} \rightarrow K^{-} \pi^{+}$was used to measure the efficiency of the DIRC for correctly identifying a charged kaon and the probability of wrongly identifying a pion as a kaon. Plots of these two variables are shown versus track momentum in Figure 2.17. The kaon efficiency remains at or above $90 \%$ for the full momentum range from 0.6 to $3.4 \mathrm{GeV} / c$. Pion mis-identification is roughly constant around $2 \%$ for momenta less that $2.4 \mathrm{GeV} / c$ and increases to just over $10 \%$ for tracks with momenta of around $3.2 \mathrm{GeV} / \mathrm{c}$. 


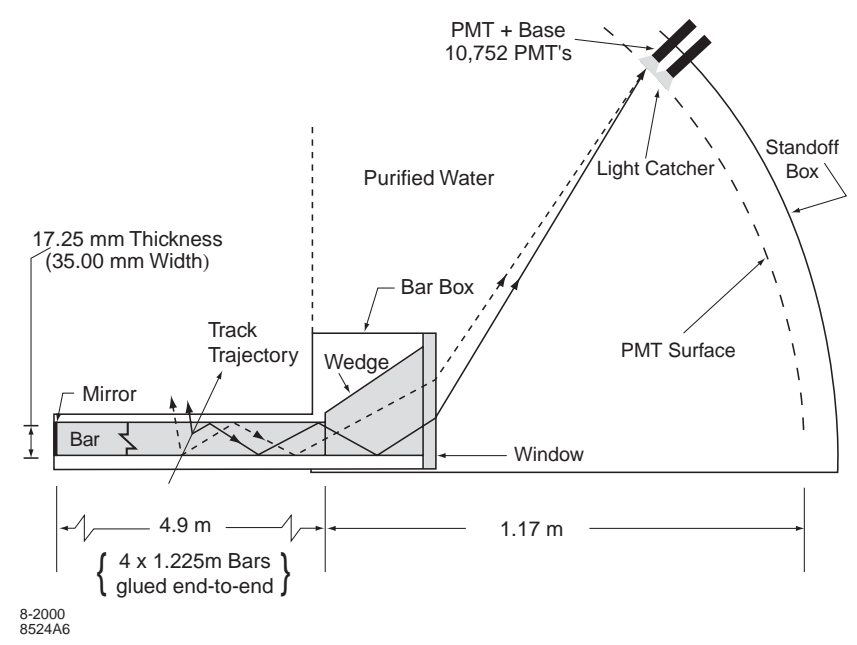

Figure 2.15: Schematic of the DIRC imaging system geometry.

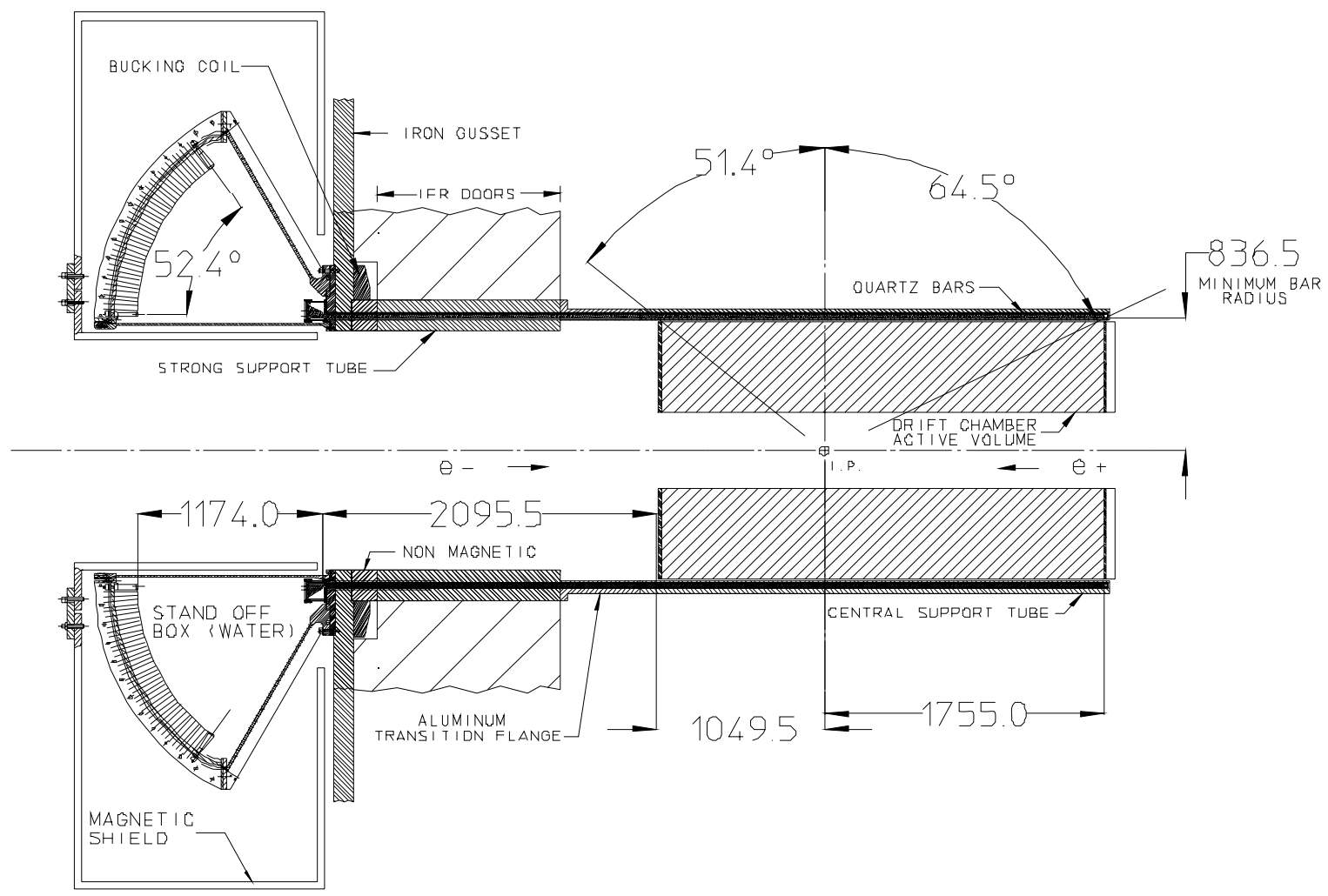

Figure 2.16: Elevation view of the layout of the DIRC system. 


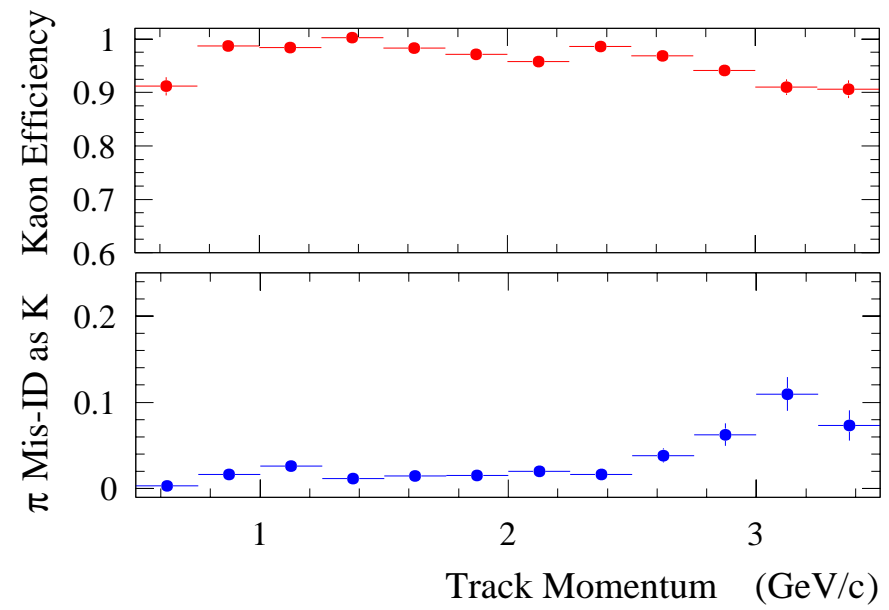

Figure 2.17: Efficiency of kaon identification by the DIRC versus track momentum is shown on the top plot. The bottom plot shows the probability that a pion will be mis-identified as a kaon as a function of track momentum. 


\subsection{The Electromagnetic Calorimeter (EMC)}

\subsubsection{EMC Theory of Operation}

The EMC is designed to absorb the energy from charged particles and high energy photons through a cascading series of electromagnetic processes generally referred to as an electromagnetic shower. These electromagnetic processes include Bremsstrahlung, ionization, pair production, Compton scattering, and the photoelectric effect. The goal is to convert as much of the energy as possible from the incoming particle or photon into longer wavelength photons that can be detected with silicon photo-diodes. Thallium-doped cesium iodide $(\mathrm{CsI}(\mathrm{Tl}))$ crystals act as a scintillator producing detectable photons through excitation and de-excitation of the thallium ions in the crystal lattice.

\subsubsection{EMC Design}

The barrel portion of the BABAR EMC consists of 5,760 thallium-doped CsI crystals arranged in 48 distinct rings (120 crystals per ring). The end-cap has 820 crystals in eight rings for a total of 6,580 crystals. The crystals have a tapered trapezoidal cross section and lengths from $29.6 \mathrm{~cm}$ to $32.4 \mathrm{~cm}$. The radiation length of the $\mathrm{CsI}(\mathrm{Tl})$ crystals is $1.85 \mathrm{~cm}$. Figure 2.18 shows a schematic diagram of a typical crystal including the readout electronics and Figure 2.19 shows the overall layout of the EMC. The EMC is surrounded by two $1 \mathrm{~mm}$-thick aluminum sheets so that 
the diodes and preamplifiers are shielded from external noise and to provide a dry, temperature controlled nitrogen atmosphere for the crystals.

\subsubsection{EMC Performance}

The energy efficiency of the EMC is measured by the rms error in the energy measurement divided by the energy $\left(\sigma_{E} / E\right)$. For the BABAR EMC this quantity is empirically described by the following

$$
\frac{\sigma_{E}}{E}=\frac{(2.32 \pm 0.30) \%}{\sqrt[4]{E(\mathrm{GeV})}} \oplus(1.85 \pm 0.12) \%
$$

where the $\oplus$ represents a sum in quadrature. Figure 2.20 shows a plot of the energy resolution of the EMC for various processes.

The angular resolution of the EMC is determined using $\pi^{0}$ and $\eta$ decays to two photons of roughly equal energy. The angular resolution is approximately $12 \mathrm{mrad}$ at low energies and $3 \mathrm{mrad}$ at high energies. Figure 2.21 shows the angular resolution $\left(\sigma_{\theta}\right)$ of the EMC for photon energies between 0 and $3 \mathrm{GeV}$.

\subsection{The Instrumented Flux Return (IFR)}

The IFR is intended to detect muons and neutral hadrons that are not stopped in the EMC. The IFR is composed of layers of Resistive Plate Chambers (RPCs) or Limited Streamer Tubes (LSTs) placed between layers of steel of increasing thickness. 


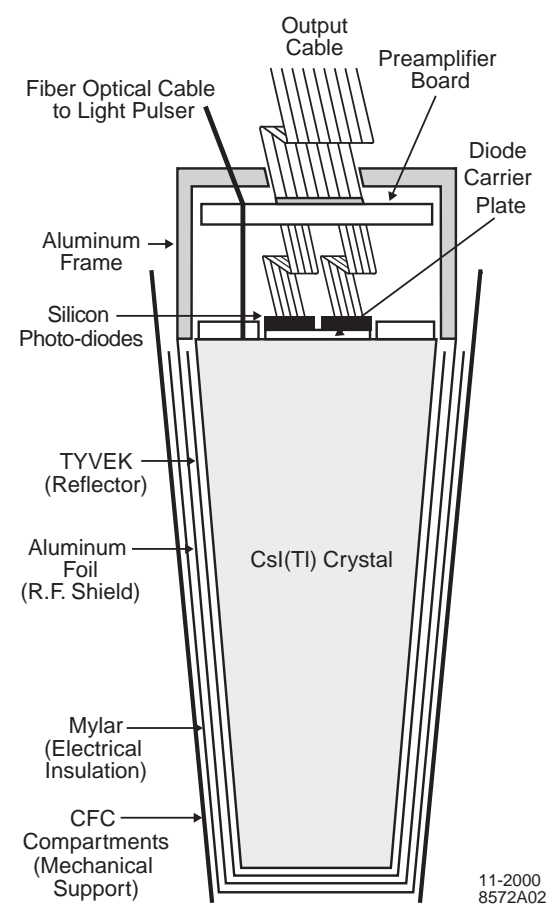

Figure 2.18: Schematic of a typical CsI(Tl) crystal (not to scale).

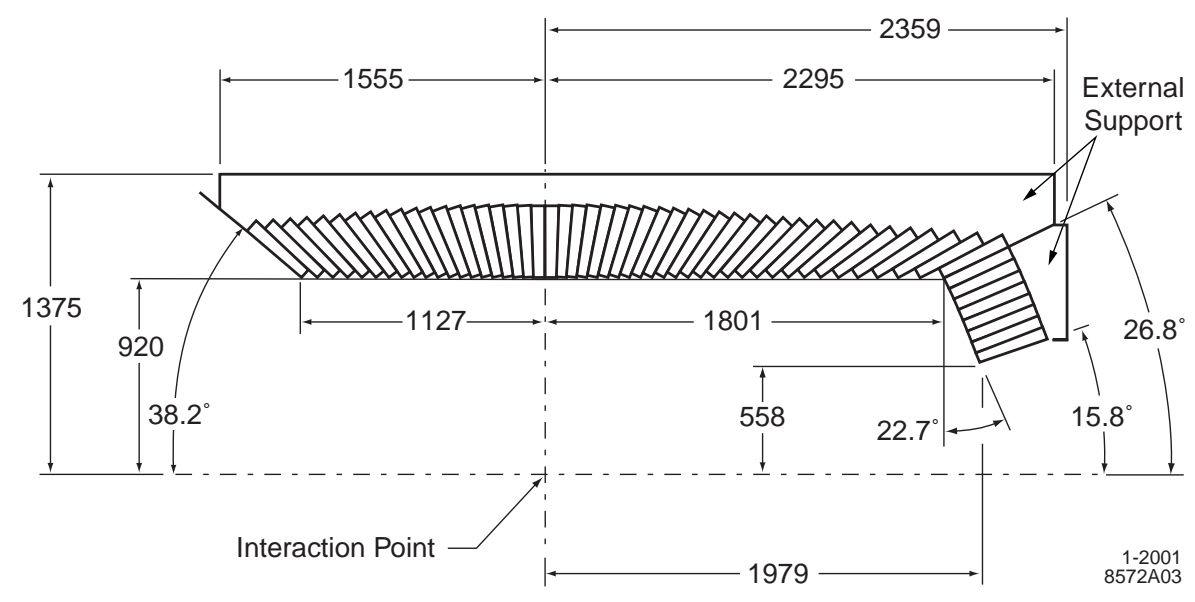

Figure 2.19: Longitudinal cross section of the EMC. All dimensions are in mm. 


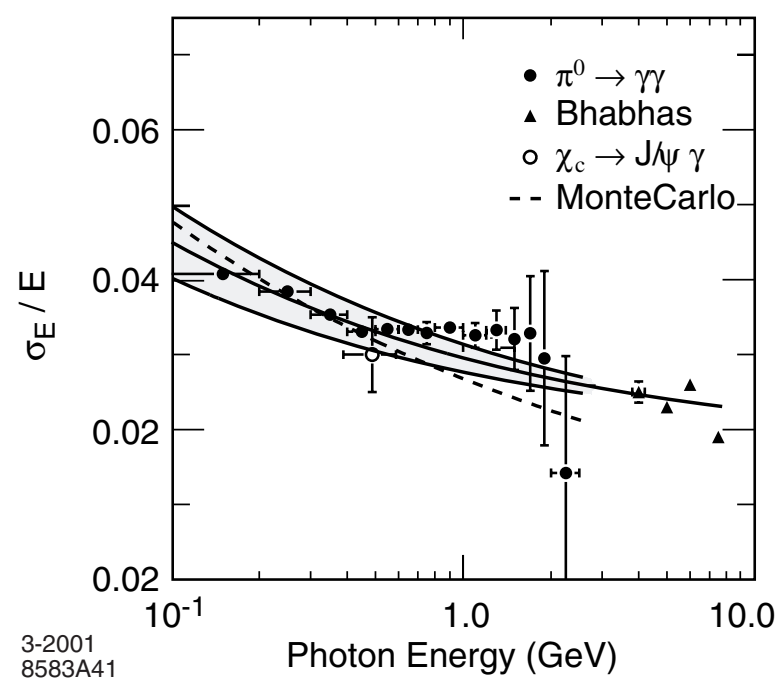

Figure 2.20: Energy resolution of the EMC for various precesses.

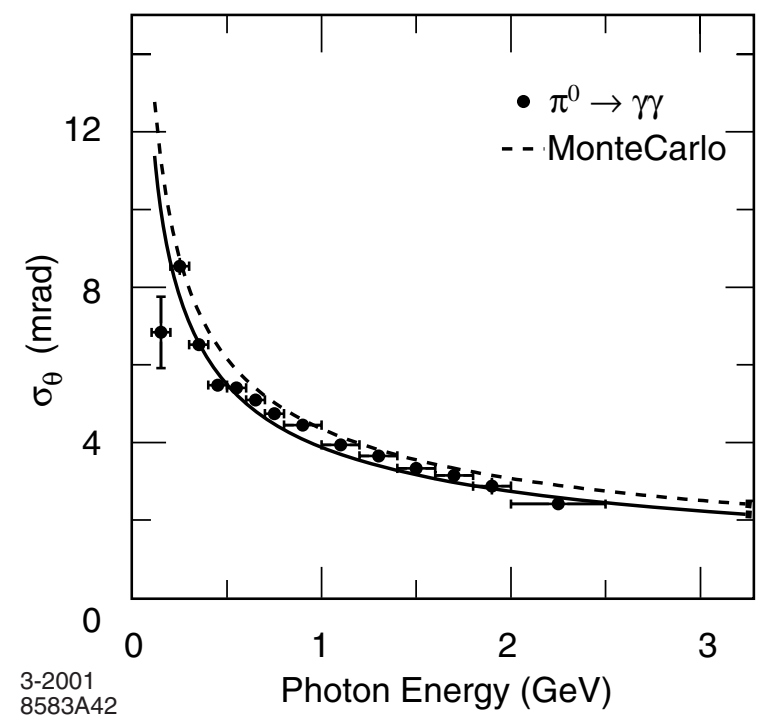

Figure 2.21: Angular resolution of the EMC for $\pi^{0} \rightarrow \gamma \gamma$. 
The BABAR detector was originally designed with only RPCs. They were sandwiched between the steel plates in the barrel and both the forward and backward end-caps (see Figure 2.22). The performance of the RPCs deteriorated rapidly once the detector began operation. The cause was believed to be overheating from the electronics. A cooling system was installed but the RPCs did not recover. The IFR portion of the forward end-cap of the detector was significantly modified in 2002 [22]. The RPCs were replaced and the electronics were moved to racks attached to the forward doors. 5 layers of $2.5 \mathrm{~cm}$ thick brass bars were installed, a $10 \mathrm{~cm}$ thick steel plate was added to the outside, and the gas system was upgraded to individually monitor each layer [23] in the entire IFR. These modifications significantly improved the operation of this portion of the IFR. The RPCs in the barrel, however, have continued to deteriorate and are being replaced with LSTs. The RPCs on the top and bottom sextants of the barrel were replaced with LSTs in 2004 and the remaining four sextants are scheduled for replacement in the summer of 2006. As part of this replacement process, 6 layers of RPCs are being replaced with brass bars.

\subsubsection{IFR Theory of Operation}

Both RPCs and LSTs operate on the same basic principle. Charged particles passing through a gas ionize the gas along their path of travel. An applied electric field causes the ionized electrons to accelerate toward the anode creating additional ionized electrons and photons through a variety of electromagnetic processes. The avalanche 
or streamer propagates to the anode.

For RPCs the cathode and anode are large sheets of bakelite coated on the back with graphite. The charge from the avalanche or streamer collects on the bakelite which has a high bulk resistivity $\left(10^{11}\right.$ to $\left.10^{12} \Omega \mathrm{cm}\right)$ to prevent the charge from dissipating too rapidly. The charge on the bakelite modifies the electric field and induces a signal on aluminum strips adjacent to the bakelite (See Figure 2.23). On one side of the RPC the readout strips are in the $\phi$ direction and on the other side they are in the $z$ direction.

As discussed in Section 2.4 for the drift chamber, an avalanche or streamer of ionized electrons produces high energy photons which can photo-ionize other electrons and create secondary avalanches leading to the breakdown of the RPC. This problem is solved in the same way by using a quenching gas to absorb photons and dissipate their energy by thermalization. The RPCs use two quenching gases: isobutane and freon. The isobutane absorbs high energy photons as in the drift chamber. Freon is electronegative and can also absorb excess electrons to prevent secondary avalanches or streamers.

The RPCs are mostly operated in streamer mode where the electric field strength is sufficiently high to produce enough charge on the bakelite that the signal can be read directly from the $\phi$ and $z$ strips without amplification. Tests are currently underway operating some of the RPCs in avalanche mode which uses a smaller electric field, a different gas mixture and requires amplifiers to read the signal. The smaller 


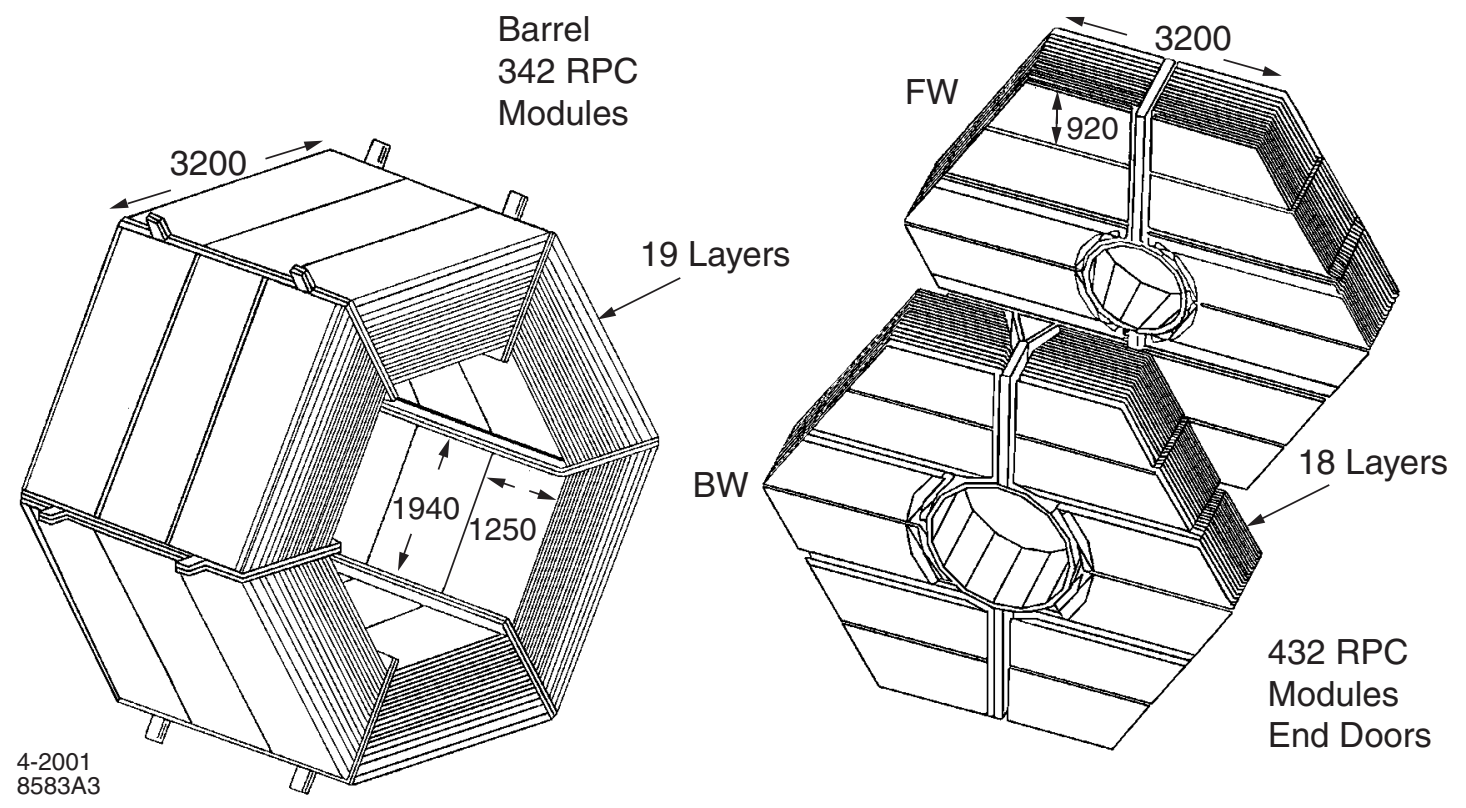

Figure 2.22: Overview of the IFR sub-detector.

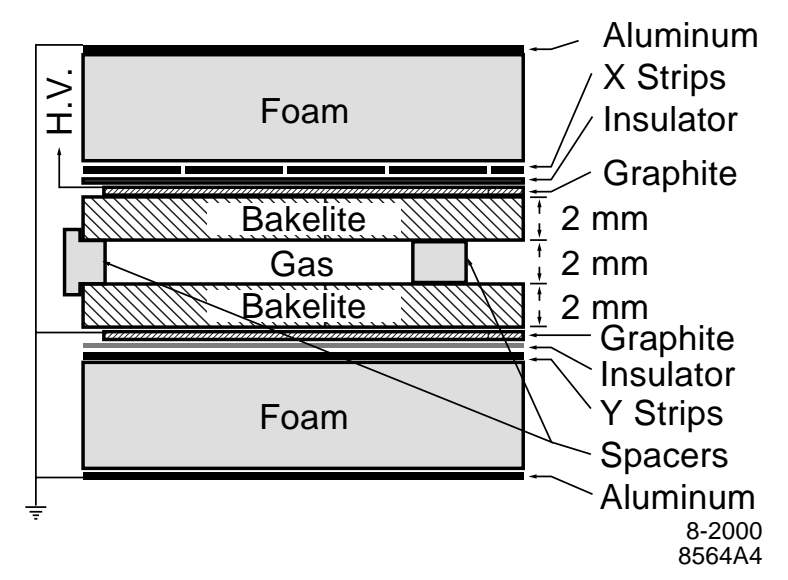

Figure 2.23: Schematic cross section of an RPC. 
field strength and reduced charge appears to produce good results with less damage to the RPCs, but the tests are still on going.

For LSTs the cathode is the outside of the tube and the anode is a wire running down the center of the tube. The method of operation is essentially the same as the RPCs except that a signal can also be read directly from the anode wire in addition to an induced signal outside the tubes.

\subsubsection{IFR Design}

The overall layout of the IFR is shown in Figure 2.22. Each sextant in the barrel has 19 layers of RPCs. During the LST upgrade 18 of the RPC layers will be removed (layer 19 is not accessible) and replaced with 12 layers of LSTs and 6 layers of brass. The forward end-cap has 16 layers of RPCs and 5 layers of brass. The backward end-cap has 18 layers of RPCs. In streamer mode the RPCs operate at a potential difference of between 6,700 and 7,800 Volts.

The gas used is a mixture of $61 \%$ argon, $35 \%$ freon, and $4 \%$ isobutane and is delivered to the detector through distribution boxes that allow the flow to each layer to be individually adjusted. The return flow from each layer is measured using a digital bubbler which counts bubbles to determine the flow rate. The added complexity of individually adjusting and measuring the flow to and from each layer was necessary due to large differences in the gas volumes and leakage rates of the individual layers. 


\subsubsection{IFR Performance}

As discussed at the beginning of this section, the RPCs initially installed have shown significantly degraded performance especially in the barrel. Figures 2.24, 2.25 and 2.26 show the RPC efficiency for the forward end-cap, the barrel and backward endcap for the period 1999 through 2004. The new RPCs in the forward end-cap have maintained their efficiency while the deterioration in the barrel RPCs has slowed significantly. Attempts at recovering the efficiency in the barrel by adjusting the HV and the gas flow rates have had limited success.

LSTs were installed in the top and bottom sextants (sextants 1 and 4) in the fall of 2004. The LSTs have maintained a relatively constant $90 \%$ efficiency for the roughly 18 months that they have been in operation.

\subsection{The Trigger and Data Acquisition (DAQ) Sys- tem}

The trigger for the BABAR detector is designed to select events of interest with a high, stable, and well understood efficiency and reject background events to prevent overloading the downstream event processing system. The trigger system has a twotier hierarchy: a Level 1 hardware trigger (L1) followed by a Level 3 software trigger (L3).

The beam crossing is essentially continuous with a 4.2 ns spacing between bunches. 


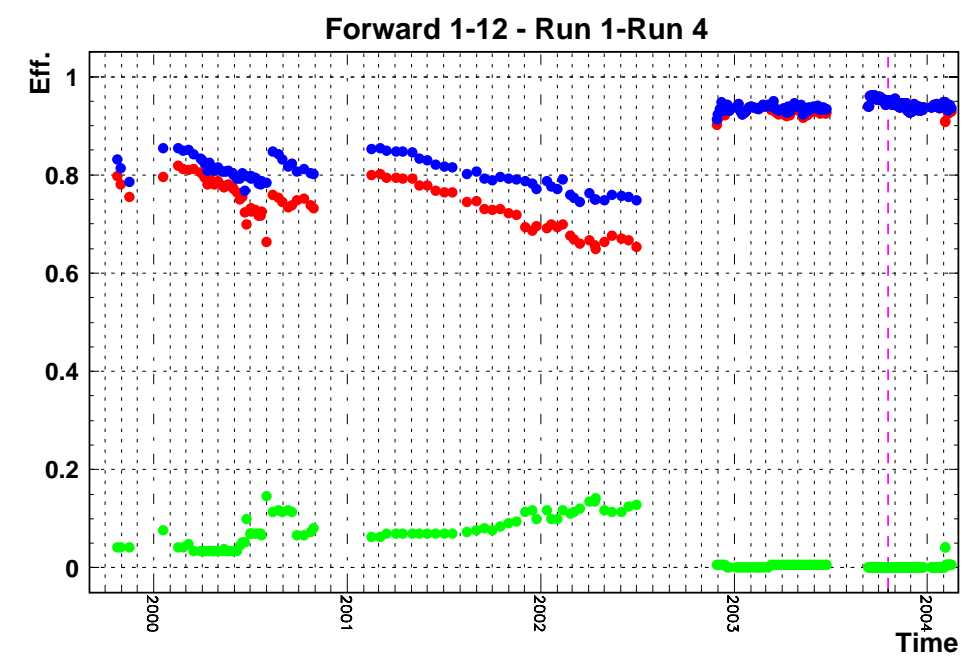

Figure 2.24: RPC efficiency in the forward end-cap from 1999 through 2004. The top (blue) curve shows the efficiency of only the RPCs that are considered operational. The middle (red) curve shows the average efficiency of all RPCs in the forward endcap. The bottom (green) curve shows the fraction of inoperative RPCs in the forward end-cap. 
The Front End Electronics (FEE) of the detector subsystems (except SVT) continuously digitize the data and buffers them for $12.9 \mu s$. Using data from the DCH, EMC, and IFR, the L1 trigger must accept or reject the event within this time window. If the event is accepted (L1 accept), all sub-detector data are read out by the data flow system and input to the L3 trigger. The FEE systems use a four event data buffer scheme to allow another L1 accept while another buffer is being readout. There is a minimum spacing of $2.7 \mu \mathrm{s}$ between successive L1 accepts. The original design for the $\mathrm{L} 1$ trigger was for a rate of $1 \mathrm{kHz}$ and the system saturates around $3.5 \mathrm{kHz}$ for typical DCH occupancies. A major upgrade to the L1 trigger was implemented in 2004 [27]. The goal of the upgrade was to reduce the background trigger rate by implementing a 3D tracking capability in the L1 trigger.

The L3 trigger software performs event reconstruction, classification, filtering, and monitoring in a farm of computers that have also been upgraded to meet the increased luminosity. It operates by refining and augmenting the selection methods used in the $\mathrm{L} 1$ trigger. The L3 trigger is expected to select events at a rate of $100 \mathrm{~Hz}$ for logging and permanent storage. 


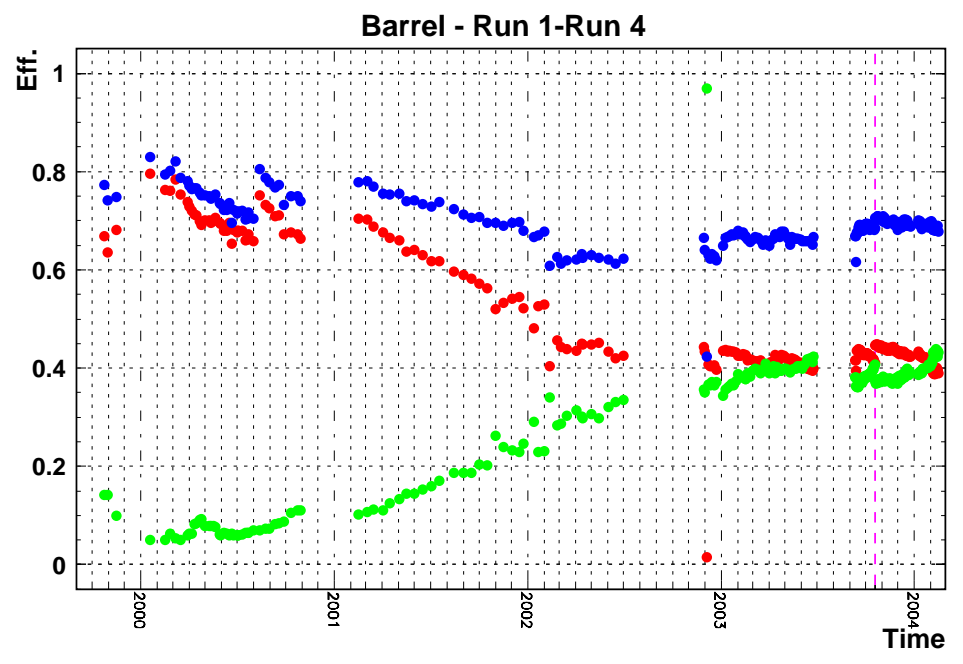

Figure 2.25: RPC efficiency in the barrel from 1999 through 2004. The top (blue) curve shows the efficiency of only the RPCs that are considered operational. The middle (red) curve shows the average efficiency of all RPCs in the barrel. The bottom (green) curve shows the fraction of inoperative RPCs in the barrel. 


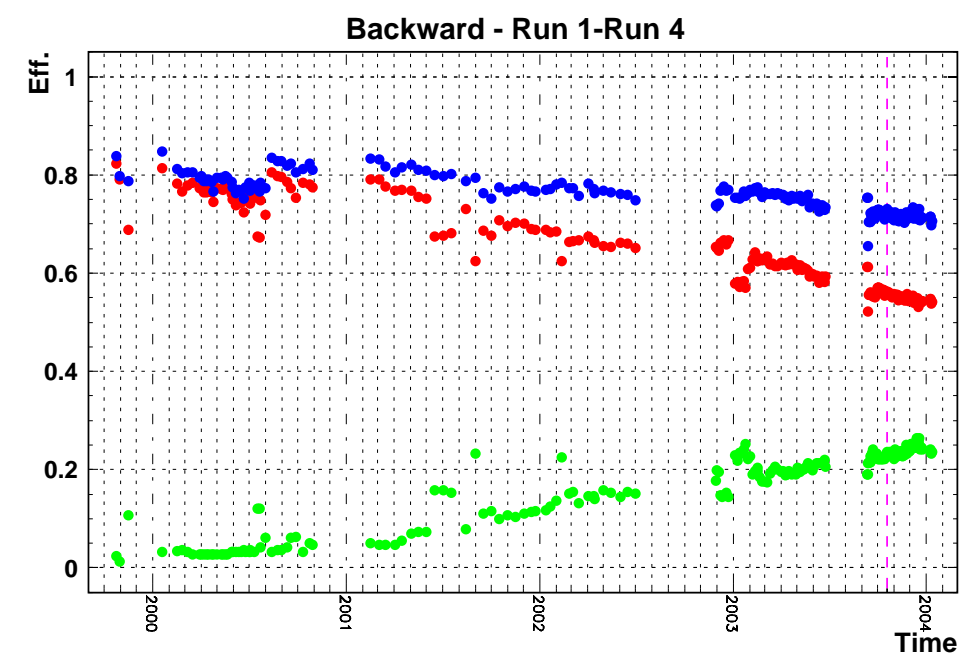

Figure 2.26: RPC efficiency in the backward end-cap from 1999 through 2004. The top (blue) curve shows the efficiency of only the RPCs that are considered operational. The middle (red) curve shows the average efficiency of all RPCs in the backward end-cap. The bottom (green) curve shows the fraction of inoperative RPCs in the backward end-cap. 


\section{Chapter 3}

\section{Analysis Techniques}

\subsection{Data and Monte Carlo Samples}

The results presented here are based on BABAR Runs 1-4 covering the period of operation from November 1999 through July 2004. The data was processed using Release 14 BABAR software package. The data sample consists of an integrated luminosity of $210 \mathrm{fb}^{-1}$ recorded at the $\Upsilon(4 S)$ resonance with a center-of-mass (c.m.) energy of $\sqrt{s}=10.58 \mathrm{GeV}$, corresponding to about 230 million $B \bar{B}$ events. An offresonance data sample of $21.6 \mathrm{fb}^{-1}$, with a center-of-mass energy $40 \mathrm{MeV}$ below the $\Upsilon(4 S)$ resonance, was used to study background contributions from light quark $e^{+} e^{-}$ $\rightarrow q \bar{q}(q=u, d, s$ or $c)$ continuum events.

Monte Carlo (MC) events were used to characterize signal and background, optimize selection criteria, and evaluate efficiencies. An MC sample of 115,000 signal 
events was generated by BABAR for this analysis. Assuming the sum of the two branching ratios for the signal channel are equal to the theoretical prediction of $1 \times 10^{-6}[5]$, and using the $B^{0}$ cross section of $1.1 \mathrm{nb}$ and the measured [9] branching ratios for $\mathcal{B}\left(K^{* 0} \rightarrow K \pi\right)=0.67$ and $\mathcal{B}\left(K^{0} \rightarrow K_{S}^{0} \rightarrow \pi \pi\right)=0.5 \times 0.68=0.34$, the MC sample represents an effective integrated luminosity of about $460,000 \mathrm{fb}^{-1}$.

For the $B^{0} \bar{B}^{0}$ and $B^{+} B^{-}$MC background event samples, a combination of the generic $B A B A R \mathrm{MC}$ and $\mathrm{MC}$ samples which simulated specific $B^{0}$ decay modes. was used. All MC samples were generated using software Release 14 processing (SP5/6), The samples of specific decay modes are presented in Table 3.1. The different categories of events referred to in Table 3.1 are discussed below in Sect. 3.6. For continuum MC events, two different generic samples from SP5/6, Release 14, were used: one for $u \bar{u}, d \bar{d}$ and $s \bar{s}$ events, and one for $c \bar{c}$ events. The number of events in the generic MC background samples and the corresponding effective integrated luminosities are summarized in the top two rows of Table 3.2 .

\subsection{Event Selection}

The selection criteria are grouped according to purpose. For example, the selection criteria related to selecting $K_{S}^{0}$ are grouped together in Section 3.2.6. For each step in the selection process the corresponding signal efficiency is given in Table 3.2. 
Table 3.1: MC samples used for Signal and $B^{0} \bar{B}^{0}$ background characterization.

\begin{tabular}{|l|c|c|}
\hline Mode & MC Sample & Number of Events \\
\hline Signal & SP-6080 & 115,000 \\
Category 1 - Same Final State: & & \\
$B^{0} \rightarrow D^{ \pm} K^{\mp}\left(D^{ \pm} \rightarrow \pi^{ \pm} K_{S}^{0}\right)$ & SP-3299 & 190,000 \\
$B^{0} \rightarrow D^{ \pm} \pi^{\mp}\left(D^{ \pm} \rightarrow K^{\mp} \pi^{ \pm} \pi^{ \pm}\right)$ & SP-2437 & 466,000 \\
$B^{0} \rightarrow K^{ \pm} \pi^{\mp} K_{S}^{0}$ & SP-1590 & $1,193,00$ \\
$B^{0} \rightarrow K_{0}^{* 0}(1430) K_{S}^{0}$ & - & 10,000 \\
Category $2-K_{S}^{0}$ plus 2 tracks & & \\
with kaon PID error: & & \\
$B^{0} \rightarrow \phi K_{S}^{0}\left(\phi \rightarrow K^{+} K^{-}\right)$ & SP-997 & 90,000 \\
$B^{0} \rightarrow f^{0} K_{S}^{0}\left(f^{0} \rightarrow K^{+} K^{-}\right)$ & SP-5180 & 115,000 \\
Category $3-K_{S}^{0}$ plus 2 tracks & & \\
with pion PID error: & & \\
$B^{0} \rightarrow D^{ \pm} \pi^{\mp}\left(D^{ \pm} \rightarrow \pi^{ \pm} K_{S}^{0}\right)$ & SP-1591 & $2,307,000$ \\
$B^{0} \rightarrow \rho^{0} K_{S}^{0}\left(\rho^{0} \rightarrow \pi^{ \pm} \pi^{\mp}\right)$ & SP-1950 & 242,000 \\
$B^{0} \rightarrow f^{0} K_{S}^{0}\left(f^{0} \rightarrow \pi^{+} \pi^{-}\right)$ & SP-3380 & 232,000 \\
$B^{0} \rightarrow \pi^{\mp} K_{2}^{* \pm}\left(K_{2}^{* \pm} \rightarrow \pi^{ \pm} K_{S}^{0}\right)$ & SP-4730 & 175,000 \\
\hline
\end{tabular}

\subsubsection{Pre-selection}

As the first step in the event selection, a pre-selection skim was used to identify a sample with the correct general signal characteristics. The skim was intended to reject obvious background while remaining essentially 100\% efficient for well reconstructed signal events contained within the detector acceptance. The BABAR collaboration has created numerous skims in order to reduce the computation time required by individual analyses.

The pre-selection for this analysis was performed using the BCCKs3Body Skim. 


\begin{tabular}{|c|c|c|c|}
\hline 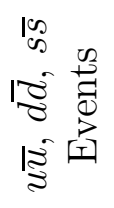 & 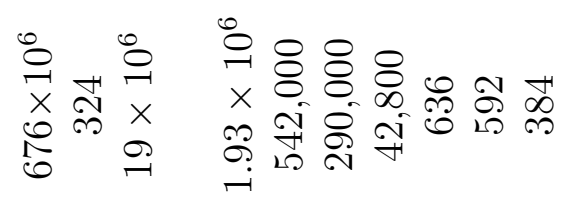 & $\infty$ & $\begin{array}{l}\stackrel{+}{0} \\
0 \\
+1 \\
0 \\
0\end{array}$ \\
\hline 论急 & 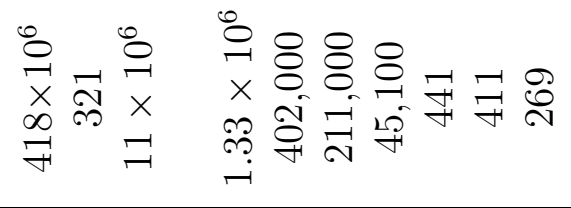 & 0 & $\begin{array}{l}8 \\
\stackrel{8}{0} \\
-1 \\
+1 \\
0 \\
0 \\
\infty\end{array}$ \\
\hline 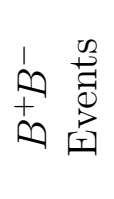 & 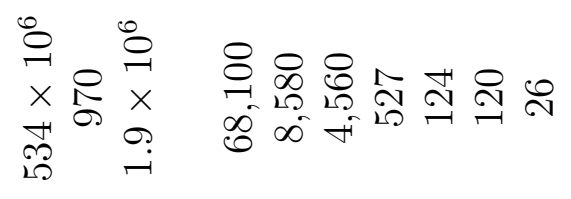 & 0 & $\begin{array}{l}\Re \\
\stackrel{0}{0} \\
+1 \\
0\end{array}$ \\
\hline 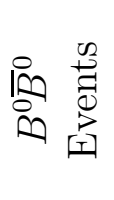 & 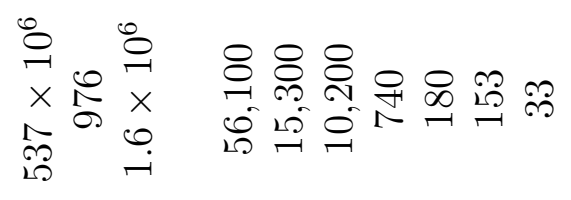 & $\infty$ & $\begin{array}{l}+1 \\
\dot{0} \\
+1 \\
20 \\
0 \\
0\end{array}$ \\
\hline 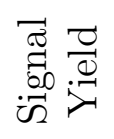 & 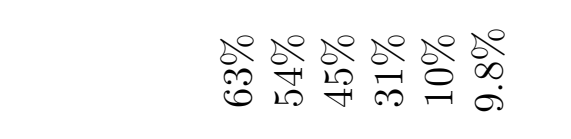 & $\stackrel{\substack{0\\
}}{1}$ & \\
\hline 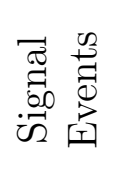 & 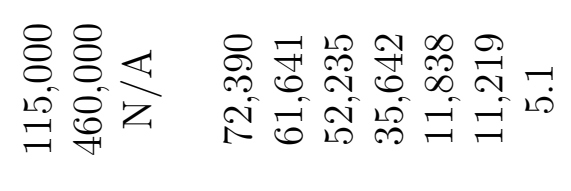 & $\begin{array}{c}\text { 유. } \\
\text { in } \\
\infty\end{array}$ & $\begin{array}{l}8 \\
\dot{0} \\
+1 \\
+1 \\
0 \\
\dot{0}\end{array}$ \\
\hline 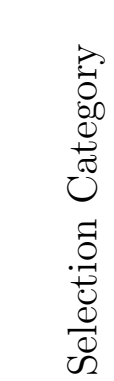 & 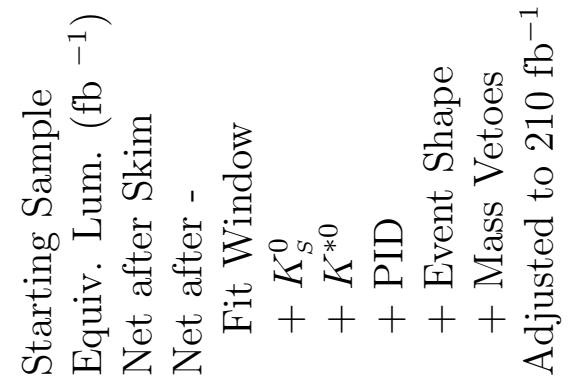 & 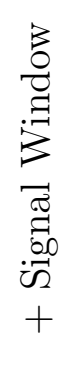 & 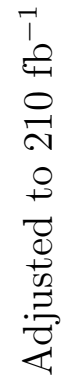 \\
\hline
\end{tabular}


This skim selected events in which a candidate $B^{0}$ decays to four charged tracks, two of which are consistent with a $K_{S}^{0}$ decay. Events were required to contain at least five charged tracks and less than $20 \mathrm{GeV}$ of total energy. The $K_{S}^{0}$ candidates were formed by combining all oppositely charged pairs of tracks, by fitting the two tracks to a common vertex, and by requiring the pair to have a fitted invariant mass, $m_{K_{S}^{0}}$, within $0.025 \mathrm{GeV} / c^{2}$ of the nominal [9] $K_{S}^{0}$ mass, assuming the two particles to be pions (this is the BABAR KsDefault list). The $K_{S}^{0}$ candidate was then combined with two other oppositely charged tracks, associated with the $K^{* 0}$ decay, to form a $B^{0}$ candidate. These latter two tracks were each required to have a distance of closest approach to the $e^{+} e^{-}$collision point of $1.5 \mathrm{~cm}$ or less in the plane perpendicular to the beam axis and $10 \mathrm{~cm}$ along the beam axis (this is the BABAR GoodTracksVeryLoose list). The so-called energy substituted mass, $m_{\mathrm{ES}}[21]$, defined by

$$
m_{\mathrm{ES}}=\sqrt{E_{\text {beam }}^{* 2}-P_{B}^{* 2}}
$$

was formed, with $P_{B}^{*}$ the c.m. momentum of the $B^{0}$ candidate and $E_{\text {beam }}^{*}$ half the c.m. energy. $m_{\mathrm{ES}}$ is used in place of the invariant mass of the $B^{0}$ because all of the beam energy goes into the $B^{0} \bar{B}^{0}$ pair and the beam energy is known with greater accuracy than the $B^{0}$ energy. $m_{\mathrm{ES}}$ was required to lie within $0.1 \mathrm{GeV} / c^{2}$ of the mass of the $B$ meson $\left(5.279 \mathrm{GeV} / c^{2}\right)$. The quantity $\Delta E$, defined by

$$
\Delta E=E_{B}^{*}-E_{\text {beam }}^{*}
$$


where $E_{B}^{*}$ is the c.m. energy of the B candidate, was required to satisfy $|\Delta E|<$ $0.35 \mathrm{GeV}$. For the calculation of $E_{B}^{*}$ and $\Delta E$, all combinations of a pion or kaon mass assignment were made for the two charged tracks not associated with the $K_{S}^{0}$ decay. (Note that the BCCKs3Body skim was not specifically developed to identify $K^{* 0} K_{S}^{0}$ events, which explains why there is no requirement for $K^{* 0}$ identification at this stage.)

\subsubsection{Particle Identification}

After the pre-selection, the two tracks assigned to the $B^{0}$ candidate but not to the $K_{S}^{0}$ were examined to test their consistency with arising from a $K^{* 0}$ meson. Of these two tracks, one was required to be identified as a kaon and the other as a pion, using the KLHTight and piLHTight criteria, respectively. The KLHTight and PiLHTight selectors are cuts to identify kaons and pions based on a likelihood method. The likelihood for each particle hypothesis is the product of likelihoods calculated for the DIRC, Drift Chamber, and the SVT:

$$
\mathcal{L}_{i}=\mathcal{L}_{i}^{D I R C} \mathcal{L}_{i}^{D C H} \mathcal{L}_{i}^{S V T}
$$

where $i$ can be either a kaon or a pion. The likelihood values for the DCH and SVT on the right side of Eq. (3.3 are determined by comparing the measured $d E / d x$ against the expected $d E / d x$ from the appropriate Bethe-Bloch formula (see citebib-LHselect for details). 
The KLHTight kaon selector requires the following to be satisfied:

- The ratio of the likelihood that the particle is a kaon to the sum of the likelihoods that it is either a kaon or a pion is greater than 0.9,

- The ratio of the likelihood that the particle is a kaon to the sum of the likelihoods that it is either a kaon or a proton is greater than 0.2 ,

- The momentum of the particle is less than $0.40 \mathrm{GeV} / c$, or else the particle is not identified as an electron. A particle is identified as an electron if the ratio of the likelihood that it is an electron to the sum of the likelihoods for all stable, charged particle hypotheses $(e, \mu, \pi, K, p)$ is at least 0.95 [31] (This corresponds to the eLHtight criterion).

The piLHTight pion selector requirements are as follows:

- The ratio of the likelihood that the particle is a kaon to the sum of the likelihoods that it is either a kaon or a pion is less than 0.5,

- The ratio of the likelihood that the particle is a proton to the sum of the likelihoods that it is either a proton or a pion is less than 0.98 ,

- The particle is not identified as an electron.

The effectiveness of PID selectors is measured by the purity of the resulting sample, the efficiency of the selector, and the fraction of other particles that meet the selector's criteria (called the "fake rate"). Sample purity is defined as the ratio of the number of truth matched tracks to the total number of kaon or pion tracks selected. The KLHTight and piLHTight selectors yield samples with purities of 90\% to $95 \%$ for kaons and $80 \%$ for pions over the full range of $m_{K^{* 0}}$. Figure 3.1 shows the sample purity versus $m_{K^{* 0}}$ for several different PID selectors using generic $B^{0} \mathrm{MC}$.

The efficiency of these selectors is defined to be the number of correctly selected kaons or pions divided by the total number of kaons or pions in the original sample. This efficiency can be measured using the $D^{*+} \rightarrow D^{0} \pi^{+}\left(D^{0} \rightarrow K^{-} \pi^{+}\right)$channel. In 

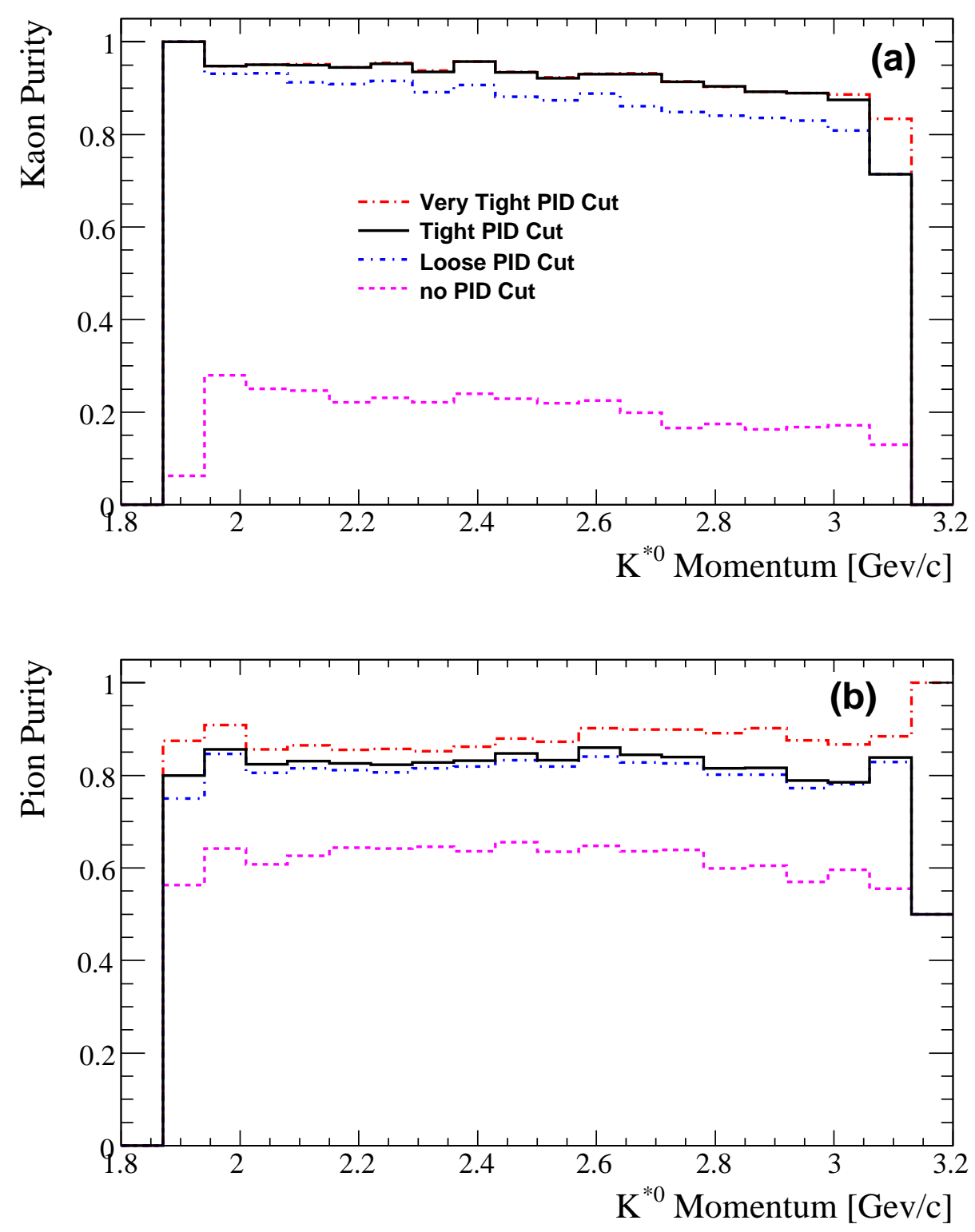

Figure 3.1: Sample purity versus $K^{* 0}$ momentum. Plot (a) shows the Kaon purity and plot (b) shows the Pion purity before and after different PID selectors have been applied. The LHTight (solid line) selectors were used in this analysis. 

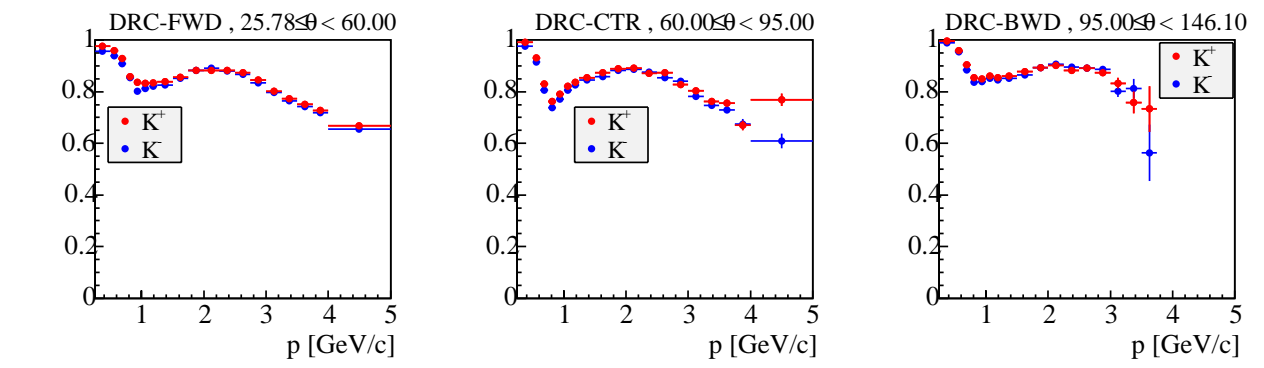

Selector : TightLHKaonMicroSelection

Dataset : run4-r16a

Figure 3.2: The kLHTight selector's kaon selection efficiency versus track momentum for three slices of $\theta$.
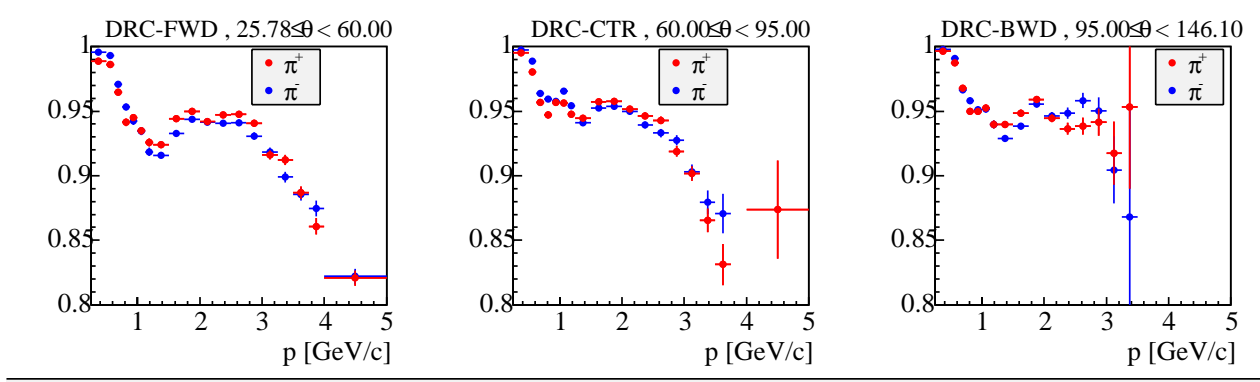

Figure 3.3: The piLHTight selector's pion selection efficiency versus track momentum for three slices of $\theta$.

this channel the identity of the kaon and pion can be determined from their charge without considering the other methods of PID. For the BABAR detector, Kaon selection efficiencies are generally greater than $80 \%$ and pion efficiencies are greater than 90\% except for high momentum tracks. Figures 3.2 and 3.3 show the kaon and pion selection efficiencies versus track momentum for three angular acceptance regions of the BABAR detector.

The fake rate is defined to be the fraction of other particles that also meet the selector's criteria. The fraction of pions that meet the kLHTight selector is generally 

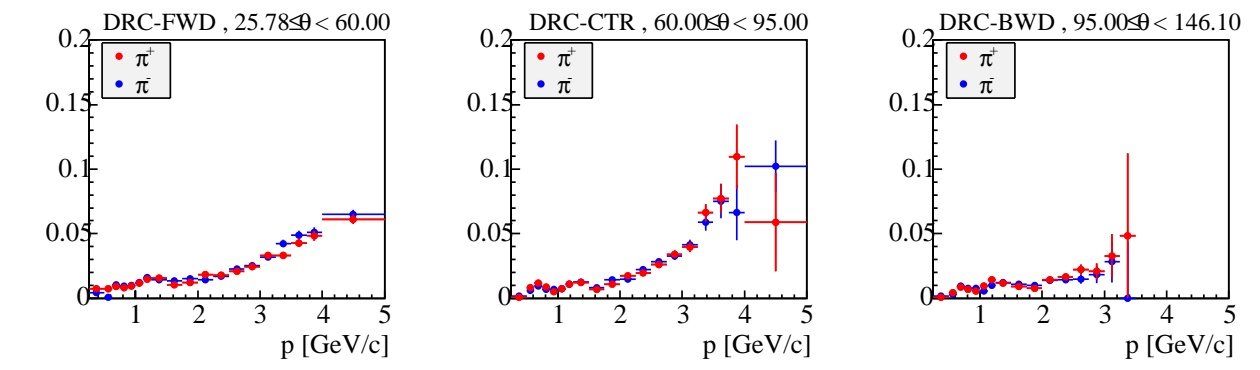

Selector : TightLHKaonMicroSelection

Dataset : run4-r16a

Tables created on 1/2/2005 (Data), 1/2/2005 (MC)

Figure 3.4: The kLHTight selector's Pion fake rate versus track momentum for three slices of $\theta$.
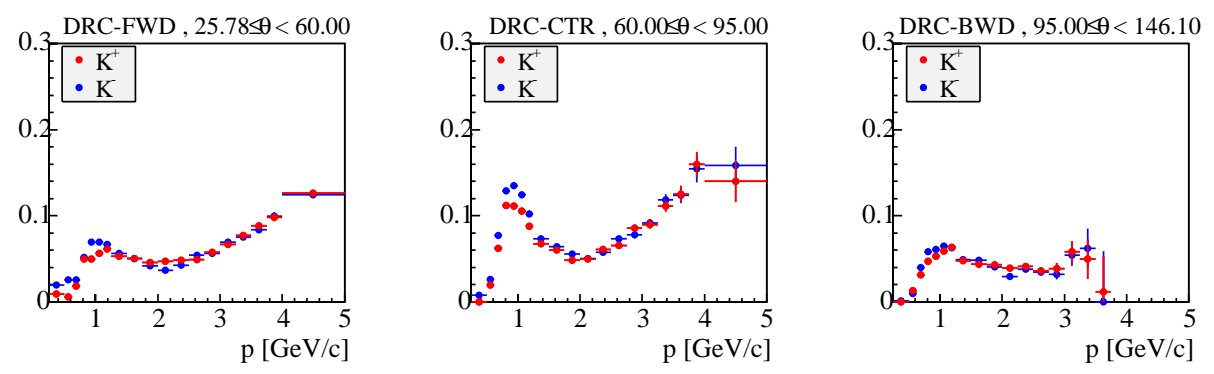

Selector : TightLHPionMicroSelection

Figure 3.5: The piLHTight selector's kaon fake rate versus track momentum for three slices of $\theta$.

less than 5\%. Figure 3.4 shows the kLHTight selector's fake rates for pions versus track momentum for three slices of angular acceptance.

The fraction of kaons that meet the piLHTight selector is generally less than $10 \%$ and is shown on Figure 3.5.

\subsubsection{Fit Window}

This study uses the Maximum Likelihood (ML) technique to determine the number of signal and background events (Sect. 3.7). The fitted experimental variables are 
$m_{\mathrm{ES}},|\Delta E|$, and the mass of the $K^{* 0}, m_{K^{* 0}} \cdot m_{K^{* 0}}$ is determined in a fit of the tracks from the $K^{* 0}$ candidate to a common vertex. We therefore established the following kinematic region inside which the ML fit is applied. We refer to this as the "fit window":

- $5.2<m_{\mathrm{ES}}<5.3 \mathrm{GeV} / c^{2}$,

- $|\Delta E|<0.15 \mathrm{GeV}$,

- $0.72<m_{K * 0}<1.20 \mathrm{GeV} / c^{2}$.

Imposing the fit window restrictions eliminated $37 \%$ of the signal MC events, $96 \%$ of the $B \bar{B}$ background MC events, and $89 \%$ of the continuum background MC events. The signal events removed correspond to poorly reconstructed events, for example, tracks which are not within the sensitive region of the detector. Figure 3.6 shows the distributions of the three fit window variables for the signal and generic $\mathrm{MC}$ samples. It is seen that the fit window restrictions are very loose. For example, virtually all well-reconstructed signal events are contained in the $m_{\mathrm{ES}}$ region between 5.27 and $5.29 \mathrm{GeV} / \mathrm{c}^{2}$ (Fig.3.6a), well within the fit window requirements for this variable. Unless specifically noted on the figure, all figures shown in Section 3.2 were generated with only the the pre-selection criteria, and the fit window requirements applied and without imposing any of the final criteria discussed below in this section.

\subsection{4 $B^{0}$ Selection Criteria}

The tracks associated with the $B^{0}$ candidate were fitted to a common vertex. The mass of the $B^{0}$, denoted $m_{B^{0}}$, was then calculated. The following criteria were 

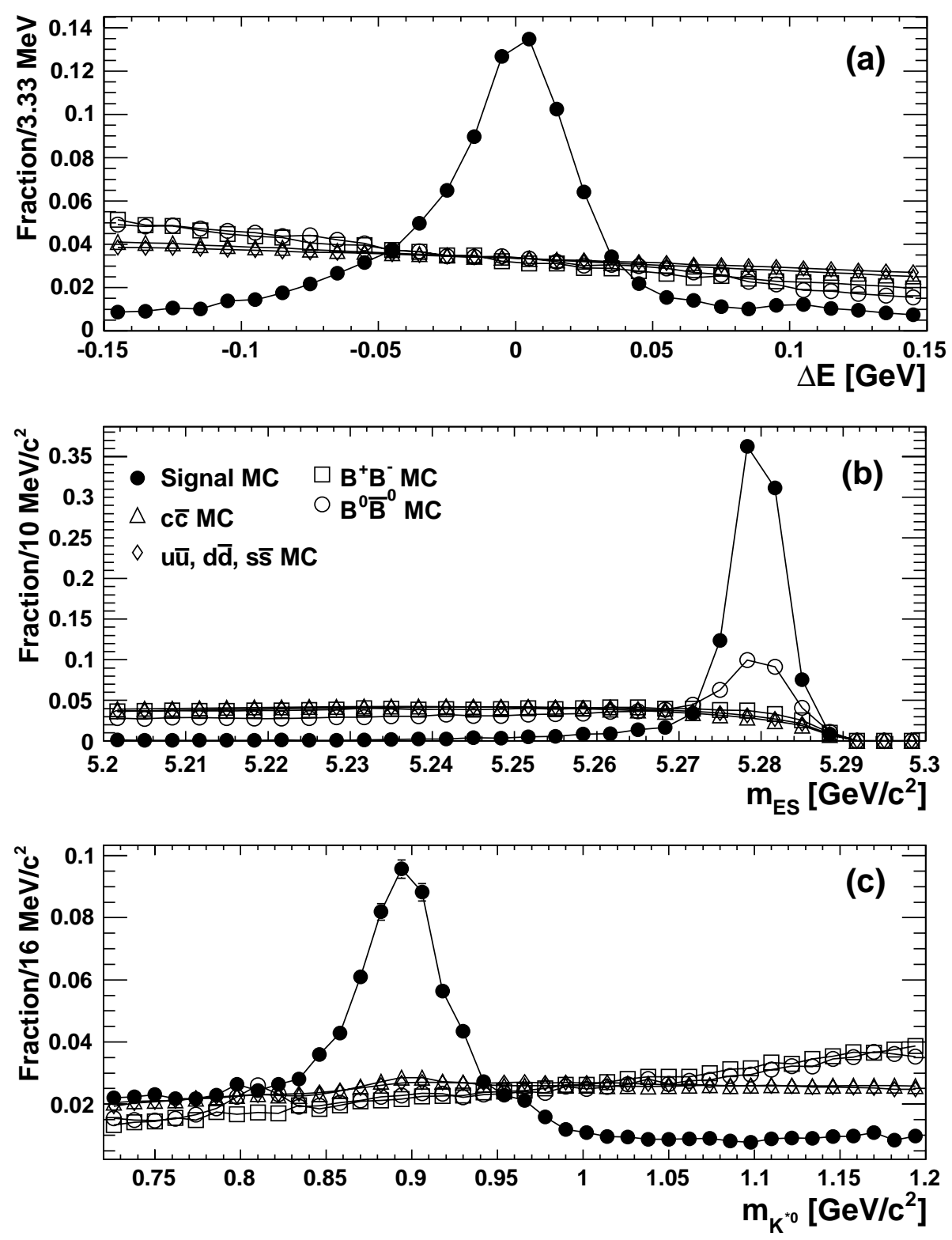

Figure 3.6: Plot (a) shows the distribution of $\Delta E$; plot (b) shows the distribution of the $m_{\mathrm{ES}}$; and plot (c) shows the distribution of the invariant mass of the $K^{* 0}$ for the five principal MC samples used in this analysis. Only the pre-selection and fit window selection criteria have been applied. 
applied:

- The $B^{0}$ vertex fit probability is greater than 0.003 ,

- $4.5<m_{B^{0}}<5.5 \mathrm{GeV} / c^{2}$.

The probability is calculated from the $\xi^{2}$ which quantifies how consistent the tracks of the vertex are with the hypothesis that they cross at that point. The value of the cut on the $B^{0}$ fit probability was optimized using the method described in Sect. 3.2.8.

The distribution of the $B^{0}$ fit probability is shown in Figure 3.7a. Only the pre-selection and fit window selection criteria have been applied.

\subsection{5 $K^{* 0}$ Selection Criteria}

In addition to the particle identification and fit window restrictions discussed in Sects. 3.2.2 and 3.2.3, $K^{* 0}$ candidates were required to satisfy the following criterion:

- $\left|\cos \theta_{\text {helicity }}\right|>0.50$, where $\theta_{\text {helicity }}$ is the angle between the $K^{* 0}$ flight direction in the $B^{0}$ rest frame and the $K^{ \pm}$momentum in the $K^{* 0}$ rest frame.

The purpose of this requirement is to take advantage of the polarization of the $K^{* 0}$ due to angular momentum conservation. The value of this cut was optimized using the method described in Sect. 3.2.8. Figure 3.7b shows the distribution of $\left|\cos \theta_{\text {helicity }}\right|$.

\subsection{6 $K_{S}^{0}$ Selection Criteria}

In addition to the $K_{S}^{0}$ pre-selection requirements mentioned in Sect. 3.2.1, $K_{S}^{0}$ candidates were subjected to the following requirements: 

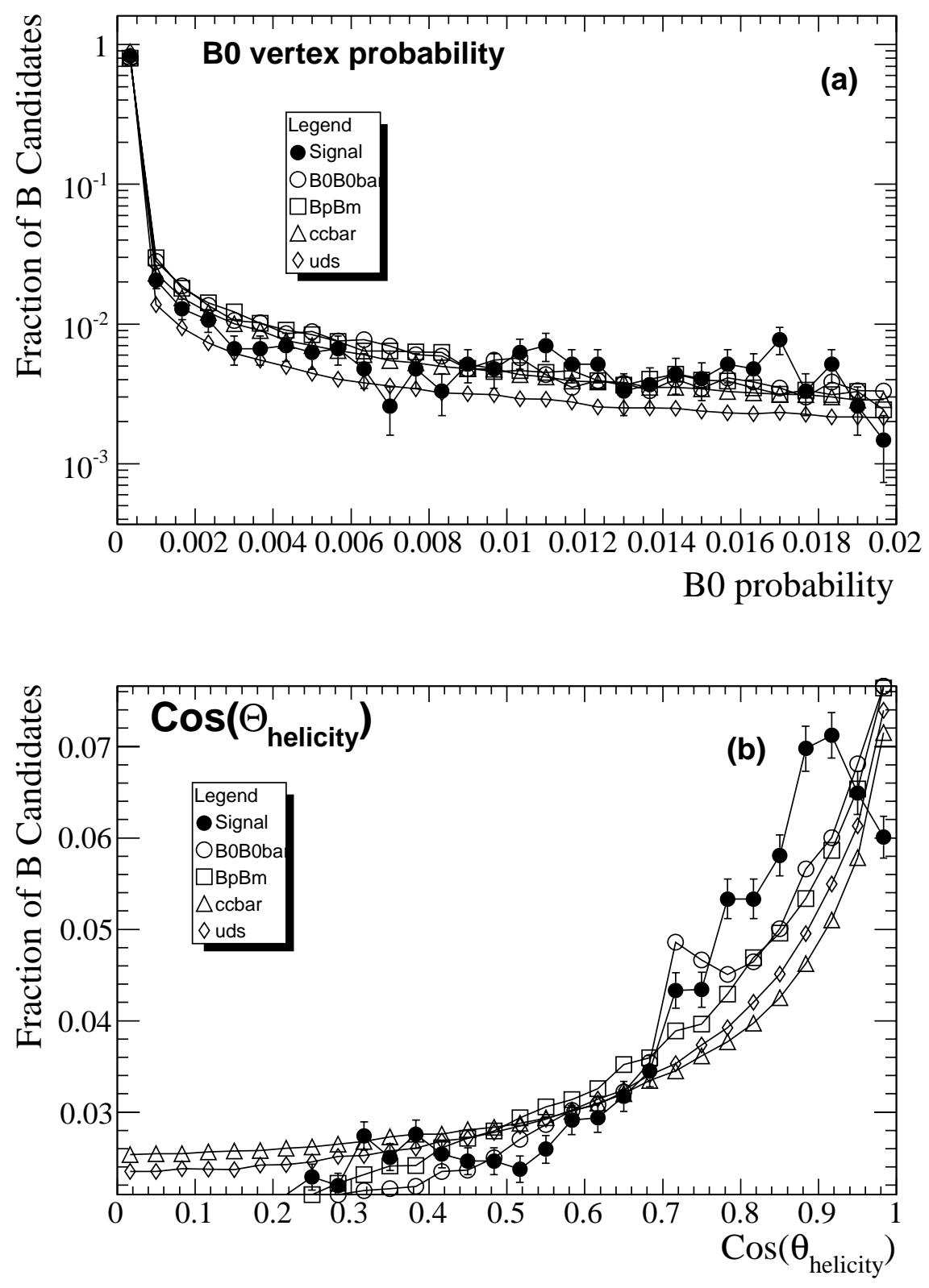

Figure 3.7: Plot (a) shows the distribution of $B^{0}$ vertex probability and plot (b) shows the distribution of the $\left|\cos \theta_{\text {helicity }}\right|$ for the five principal MC samples used in this analysis. Only the pre-selection and fit window selection criteria have been applied. 
- The $K_{S}^{0}$ vertex fit probability is greater than 0.06 ,

- $m_{K_{S}^{0}}$ is within $0.0105 \mathrm{GeV} / c^{2}$ of the peak of the reconstructed $K_{S}^{0}$ mass distribution (at $0.4979 \mathrm{GeV} / c^{2}$ ),

- The $K_{S}^{0}$ decay length significance, defined by the distance between the $K^{* 0}$ and $K_{S}^{0}$ decay vertices divided by the uncertainty on that quantity, is greater than 3. Defining the decay length vector, $d$, to be the position vector between the $K^{* 0}$ and $K_{S}^{0}$ decay vertices, and the covariance matrices of the the $K^{* 0}$ and the $K_{S}^{0}$ vertices to be $c_{1}$ and $c_{2}$, the decay length significance $S$ is given by $S \equiv \sqrt{d^{t} \cdot\left(c_{1}+c_{2}\right)^{-1} \cdot d}$,

- $\left|\cos \theta_{K_{S}^{0}}\right|>0.997$, where $\theta_{K_{S}^{0}}$ is the angle between the decay length vector and the $K_{S}^{0}$ momentum direction, evaluated in the laboratory frame.

These selection criteria were designed to take advantage of the fact that the $K^{* 0}$ vertex is almost coincident with the $\mathrm{B}$ vertex due to the short lifetime of the $K^{* 0}$, whereas the $K_{S}^{0}$ has a relatively long decay length.

The values of these cuts were optimized using the method described in Sect. 3.2.8. The distributions of these four variables are shown in Figures 3.8 and 3.9.

\subsubsection{Event Shape Selection Criteria}

To separate signal events from the continuum background, we apply cuts on event shapes, i.e. on global momentum properties of the events. $B^{0}$ mesons in $\Upsilon(4 S)$ decays are produced almost at rest. Therefore, the $B^{0}$ decay products are essentially isotropic in the event c.m. In contrast, continuum $e^{+} e^{-} \rightarrow q \bar{q}$ events at the $\Upsilon(4 S)$ energy are characterized by back-to-back two-jet-like event structures, because of the relatively small masses of $u, d, s$ and $c$ quarks. 

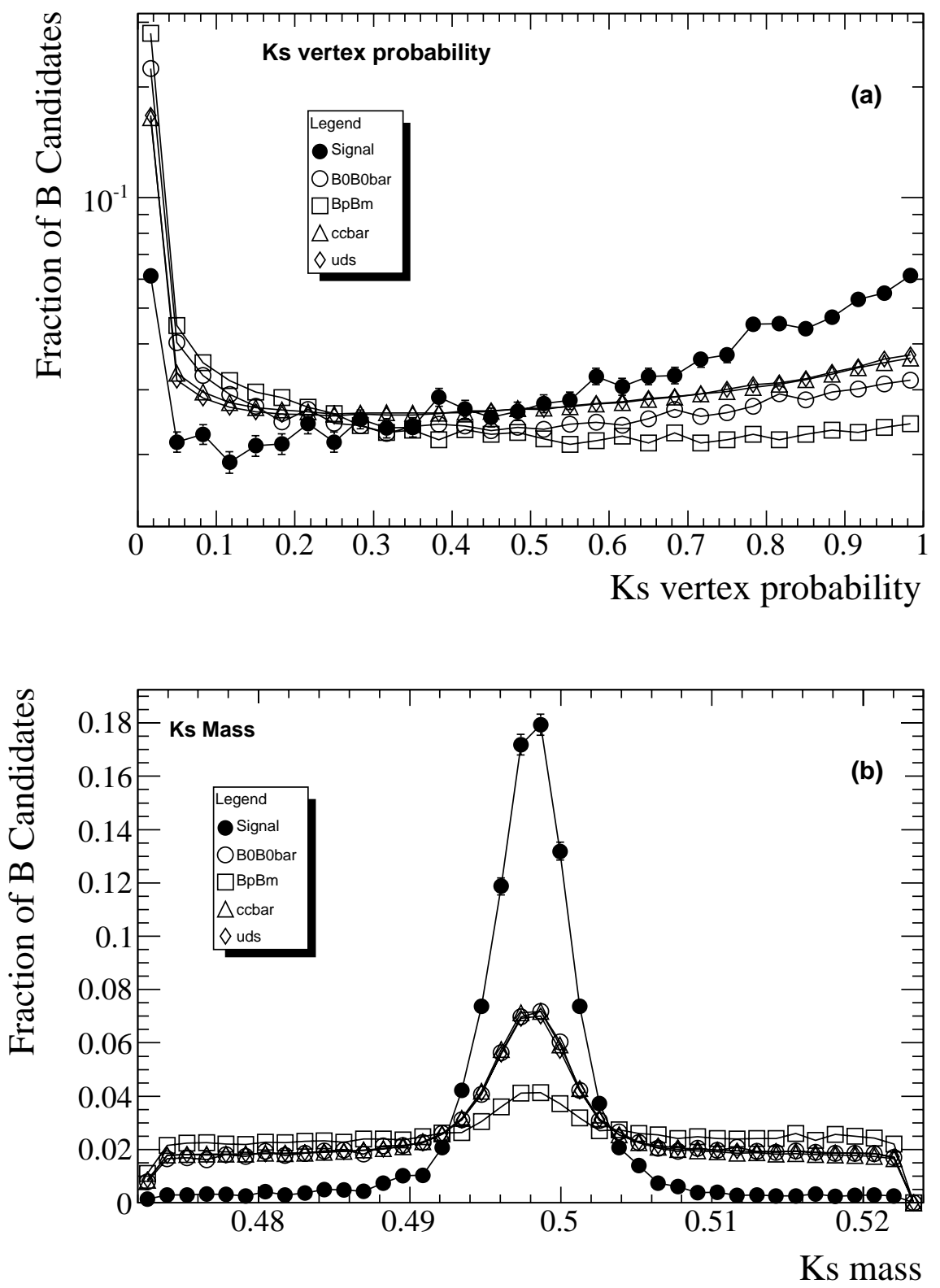

Figure 3.8: Plot (a) shows the distribution of $K_{S}^{0}$ vertex fit probability and plot (b) shows the distribution of the $K_{S}^{0}$ mass for the five MC samples used in this analysis. Only the pre-selection and fit window selection criteria have been applied. 

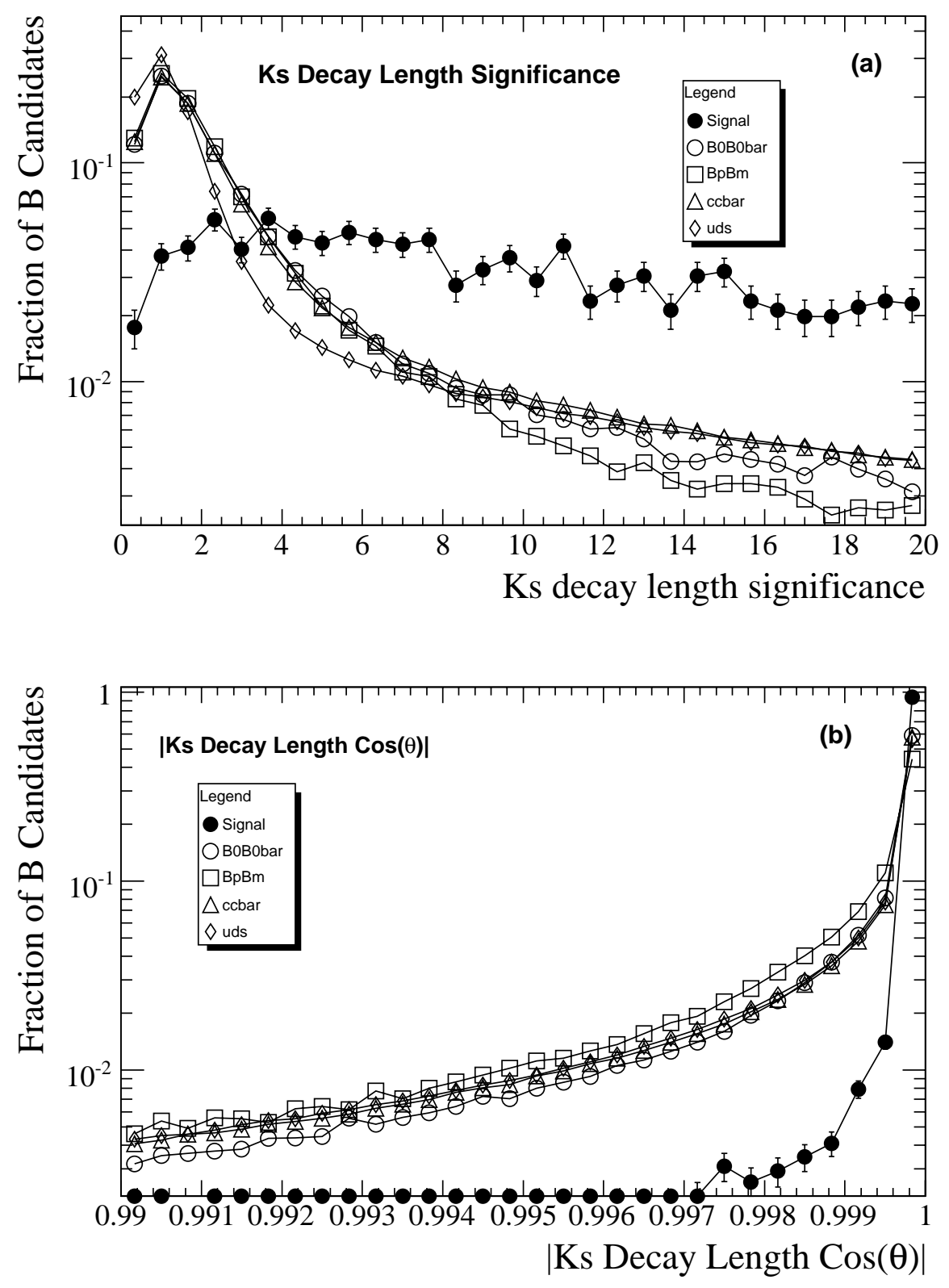

Figure 3.9: Plot (a) shows the distribution of $K_{S}^{0}$ decay length significance and plot (b) shows the distribution of the absolute value of the $K_{S}^{0}$ decay length $\operatorname{Cos}(\theta)$ for the five MC samples used in this analysis. Only the pre-selection and fit window selection criteria have been applied. 
As a means to separate signal from background events using event shapes, we rely, in part, on the Legendre polynomial-like terms $L_{0}$ and $L_{2}$ defined by

$$
L_{0}=\sum_{\text {r.o.e. }} p_{i} \quad ; \quad L_{2}=\sum_{\text {r.o.e. }} \frac{p_{i}}{2}\left(3 \cos ^{2} \theta_{i}-1\right)
$$

where $p_{i}$ is the magnitude of the 3 -momentum of a particle and $\theta_{i}$ is its polar angle with respect to the thrust [32] axis, with the latter determined using the candidate $B^{0}$ decay products only. The sums in Eq. (3.4) are performed over all particles in the event not associated with the $B^{0}$ decay ("rest-of-event" or r.o.e.). $L_{0}$ and $L_{2}$ are evaluated in the c.m. frame.

Fig. 3.10 shows scatter plots of $L_{0}$ versus $L_{2}$ for signal and continuum MC events. To separate the two event classes, we use a line defined by $\mathcal{F}=0.374 L_{0}-1.179 L_{2}$ (see the solid line in Fig. 3.10, where $\mathcal{F}=0.15$ ). The coefficients of $L_{0}$ and $L_{2}$ were determined in a separate analysis [35] by using the Fisher Discriminant method.

The following event shape criteria were applied:

- Fisher Discriminant $\mathcal{F}>0.15$,

- $\left|\cos \theta_{\text {thrust }}\right|<0.55$, where $\theta_{\text {thrust }}$ is the angle between the momentum of the $B^{0}$ candidate and the thrust axis, evaluated in the c.m. frame, with the thrust axis in this case determined using all particles in the event except those associated with the $B^{0}$ candidate (i.e. the r.o.e. particles). Note that this differs from the definition of the thrust axis used to determine $L_{0}$ and $L_{2}$.

The values of these cuts were optimized using the method described in Sect. 3.2.8.

The two plots on the left side of Figure 3.11 (plots a and c) show the distributions of these two variables with only the pre-selection and fit window cuts applied. Because 

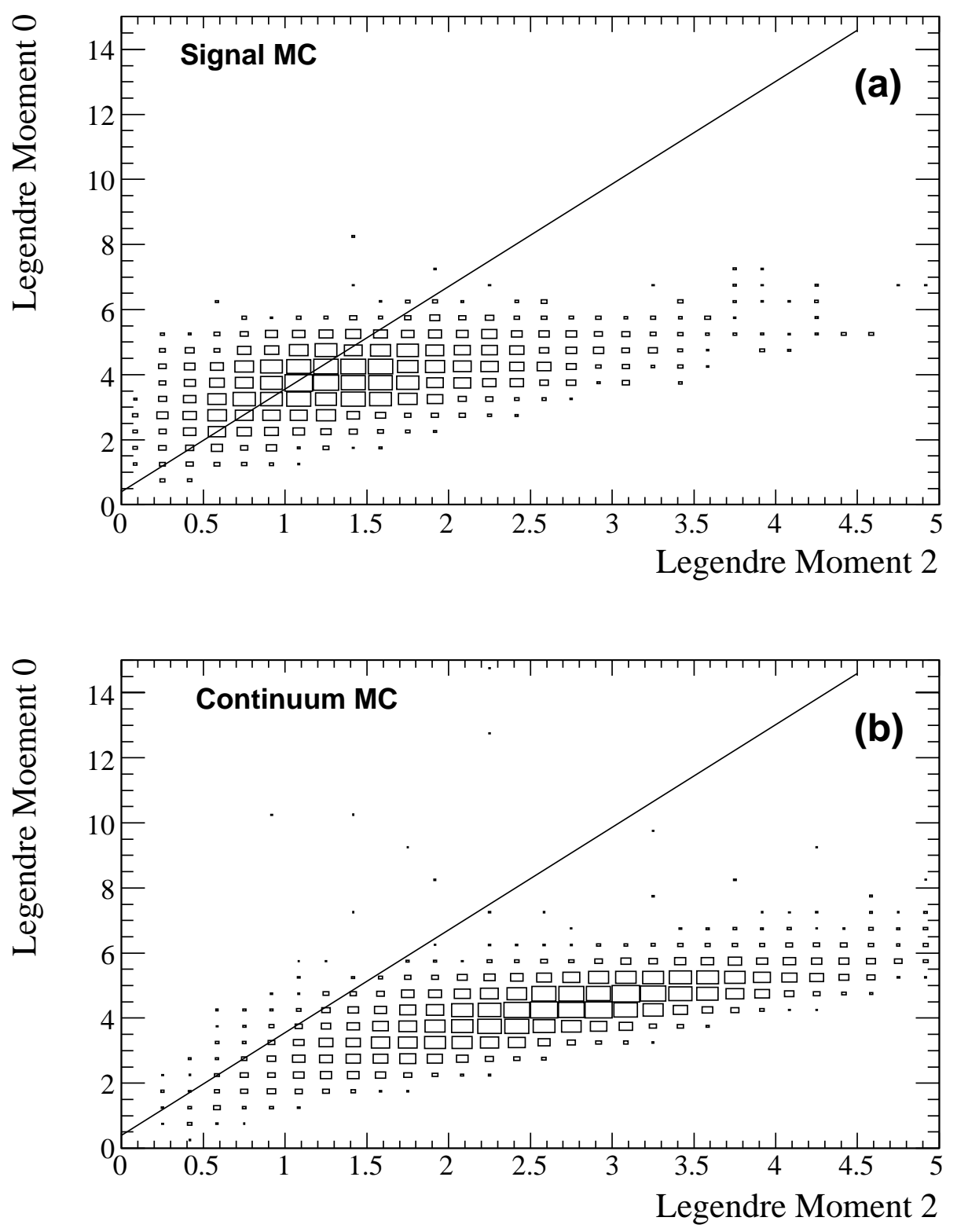

Figure 3.10: Two dimensional plots of $L_{0}$ versus $L_{2}$. Plot (a) is signal MC events and plot (b) is continuum MC events. The diagonal line is $\mathcal{F}=0.374 L 0-1.179 L 2$, where $\mathcal{F}=0.15$ No selection criteria were applied in these plots. 
these two variables are correlated we also show the Fisher distribution with a cut on the thrust and the thrust distribution with a cut on the Fisher (right side of fig. 3.11, plots $b$ and $d)$. 

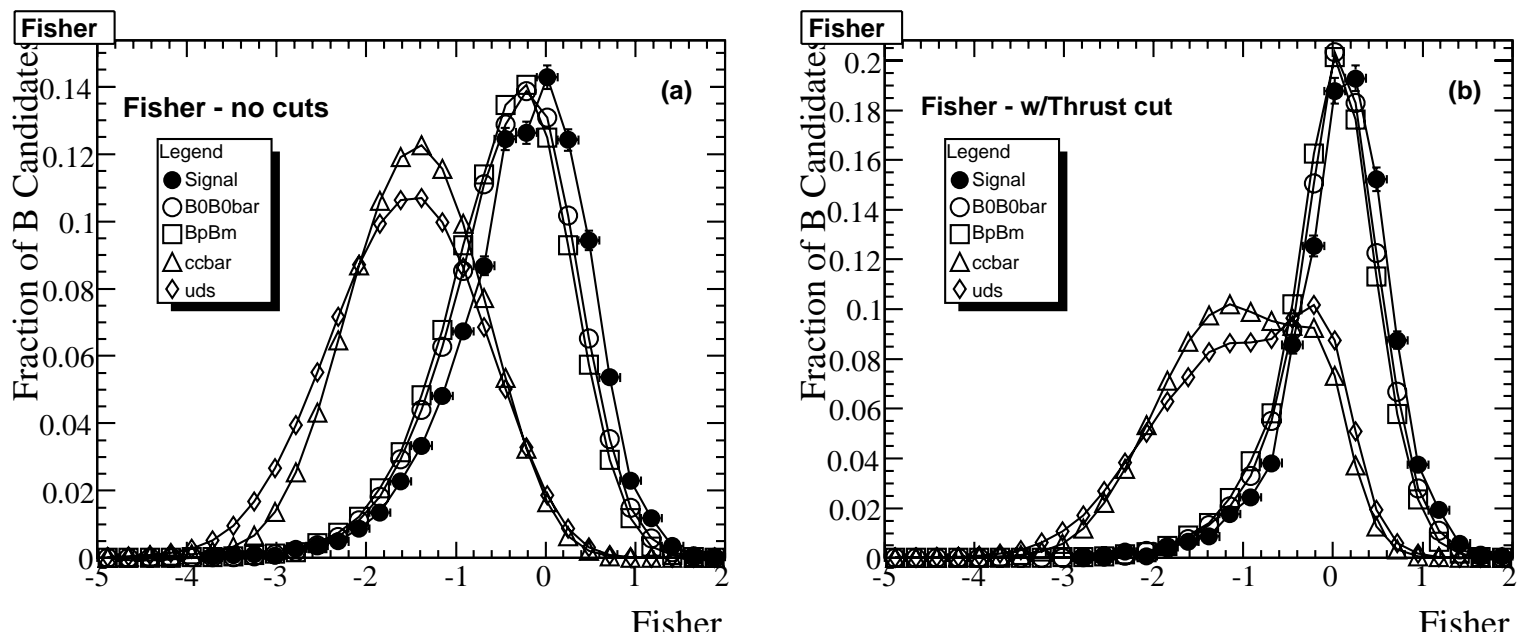

Fisher

Fisher
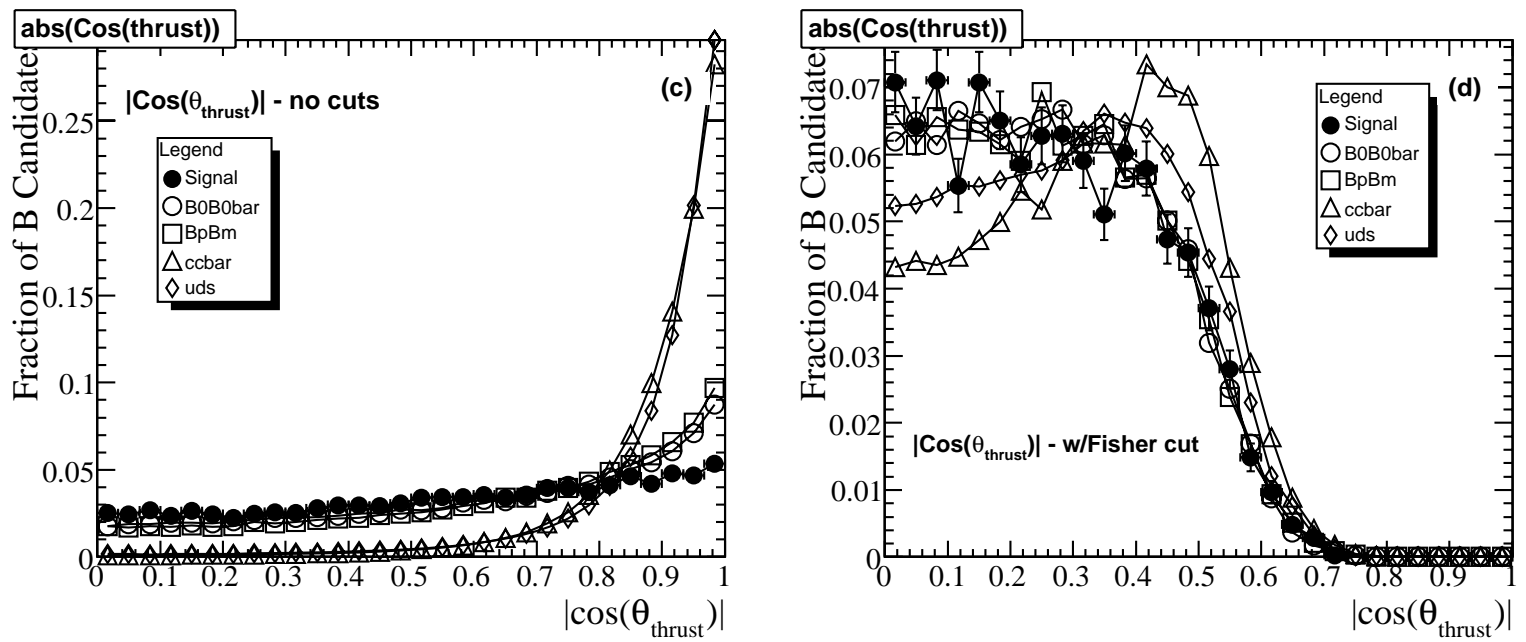

Figure 3.11: Plot (a) shows the distribution of the Fisher discriminant with only the pre-selection and fit window selection criteria applied. Plot (b) shows the distribution of the Fisher discriminant with the cut on the absolute value of cos of the thrust angle. Plot (c) shows the distribution of the absolute value of cos of the thrust angle with only the pre-selection and fit window selection criteria applied. Plot (d) shows the distribution of the absolute value of cos of the thrust angle with the cut on the Fisher discriminant. 


\subsubsection{Optimization of Selection Criteria}

The values of the cuts described in Sects. 3.2.4 - 3.2.7 were optimized using the following procedure. The values of the cuts were initially set so that essentially no well reconstructed signal MC event was rejected, based on the distributions shown in Figs. 3.7-3.9 and 3.11. The number of selected signal and background events normalized to the integrated luminosity of the data, $N_{s i g}$ and $N_{b k g}$, were then used to minimize the estimated upper limit on the $B^{0} \rightarrow \bar{K} * * 0 K^{0}$ branching ratio $\mathcal{B}$ using the following expression:

$$
\mathcal{B}<\left(\frac{N_{\text {sig }}^{\text {lim }}}{N_{\text {sig }}}\right) \mathcal{B}_{\text {theory }}
$$

where $N_{\text {sig }}^{\text {lim }} \equiv N_{\text {sig }}+1.64 \sigma$, with $\sigma=\sqrt{N_{\text {sig }}+N_{\text {bkg }}}$, and where $\mathcal{B}_{\text {theory }}=0.5 \times 10^{-6}$ is the predicted branching ratio [5]. The value of each selection variable was then varied by a fixed amount designed to cover the expected range of reasonable values using 10 increments, with the other variables held constant at their initial values. For each value of the variable, the upper limit defined by Eq. (3.5) was re-calculated.

Each variable was then set equal to the value which yielded the minimum upper limit estimate. If multiple values of the variable yielded the same estimate, the least restrictive cut value was selected.

The process described in the previous paragraph was then repeated, this time by varying the value of each variable in five equal increments above and below its new initial value. The process was repeated until a stable minimum was found for each of the eight variables. Four iterations of the procedure were required. Figure 3.12 
shows the final set of optimization curves. The arrows indicate the final, optimized values for each selection variable. These values were already reported in Sects. 3.2.4 -3.2 .7 .

\subsubsection{Multiple $B^{0}$ Candidates}

If more than one $B^{0}$ candidate survives the selection procedure it is necessary to select which candidate to use in the fit. As shown in Figure 3.13 over 96\% (656 out of 682 ) of the events that pass all of the selection criteria have only one $B^{0}$ candidate. Of the events with multiple $B^{0}$ candidates $92 \%$ (24 out of 26 ) have two $B^{0}$ candidates and the remaining 2 events have three $B^{0}$ candidates. No events that survived the selection procedure have more than three $B^{0}$ candidates. For the $3.8 \%$ of events where more than one $B^{0}$ candidate survived the selection procedure, only the candidate with the largest $B^{0}$ vertex fit probability was retained and used in the fit.

\subsection{Event Selection Summary}

In addition to the criteria described in this section, two additional cuts based on "mass vetoes" are also applied. The mass vetoes are targeted to reduce the contributions or specific B background categories and have a minimal impact on the overall selection efficiencies. These cuts are described below in Sect. 3.6.

The selection criteria eliminate $99.78 \%$ and $99.97 \%$ of the $B \bar{B}$ and continuum 

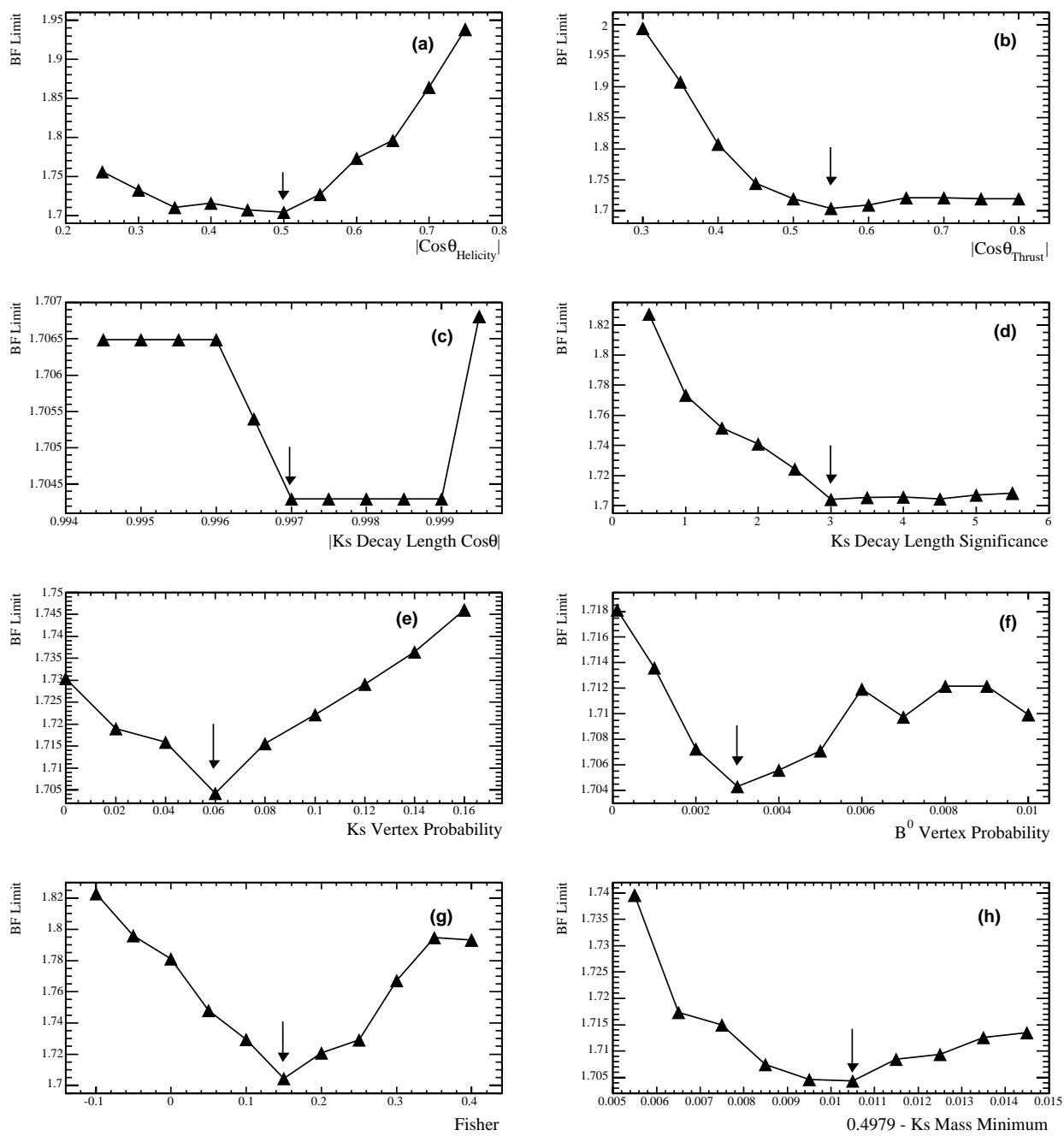

Figure 3.12: Cut optimization plots showing the BF limit vs. each of the variables used as selection criteria. 


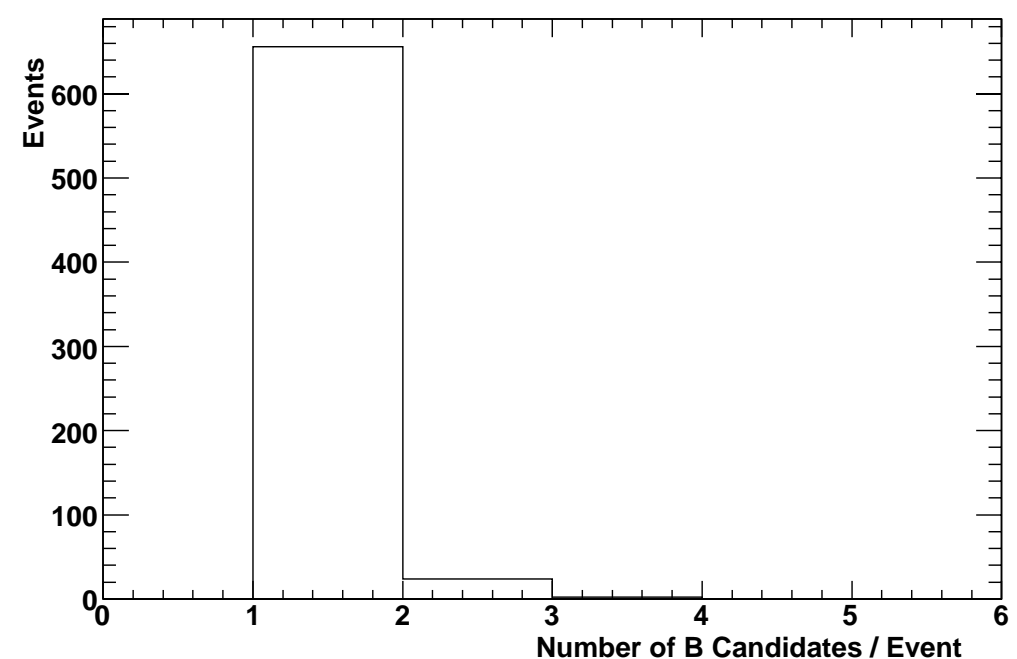

Figure 3.13: Histogram of the number of $\mathrm{B}$ candidates per event after all cuts are applied.

background events, respectively and have an overall signal efficiency of 9.8\%. The most important cuts are the PID and Event Shape requirements. The selection efficiencies for each group of cuts are given in Table 3.2.

\subsection{Expected Sensitivity}

In order to obtain an estimate of the number of expected signal events, we evaluated the expected sensitivity of this analysis. For the purposes of establishing this sensitivity, we defined the following "signal window":

- $5.271<m_{\mathrm{ES}}<5.286 \mathrm{GeV} / c^{2}$,

- $|\Delta E|<0.039 \mathrm{GeV}$, 
- $0.82<m_{K^{* 0}}<0.97 \mathrm{GeV} / c^{2}$.

The signal window criteria for $m_{\mathrm{ES}}$ and $\Delta E$ were determined using the optimization procedure described in Sect. 3.2.8, with all the selection cuts of Sect. 3.2 at their final values. For $m_{K^{* 0}}$, the signal window criteria were set at the $K^{* 0}$ mass $\pm 1.5 \times$ the width of the reconstructed $K^{* 0}$ mass peak. Note that the main results of this study are based on a Maximum Likelihood fit applied within the fit window defined in Sect. 3.2.3 and do not utilize this signal window.

The signal window expectations for signal and background MC events are presented in the bottom two rows of Table 3.2. Based on these results and using Eq. (3.5), we estimate the $90 \%$ C.L. upper limit sensitivity of this analysis to be $\mathcal{B}\left(B^{0} \rightarrow \overline{K^{* 0}} K^{0}\right)+\mathcal{B}\left(B^{0} \rightarrow K^{* 0} \overline{K^{0}}\right)<1.6 \times 10^{-6}$.

\subsection{Continuum Monte Carlo Evaluation}

To confirm that the generic continuum MC accurately characterizes the data, the off-resonance data sample was plotted against the $q \bar{q} \mathrm{MC}$ samples for several parameters of interest. All MC samples were normalized to the off-resonance luminosity of $21.6 \mathrm{fb}^{-1}$. Figure 3.14 shows plots of $m_{\mathrm{ES}}, m_{K^{* 0}}$, and $m_{K s}$ comparing the off-peak data sample (histogram) with the two generic $q \bar{q}$ samples (stacked histograms). The plots on the left side of Figure 3.14 (plots a, c, and e) have no selection criteria applied. As can be seen from the figure, the differences between the MC and the data samples are minor. A second set of plots showing the same parameters after the 
selection criteria described in Section 3.2 have been applied are shown on the right side of Figure 3.14. Again, the differences are relatively minor.

\subsection{Background Evaluation}

The residual background after the cuts of Sect. 3.2 have been applied can be broken into two general classes: combinatoric background, without peaking in the $m_{\mathrm{ES}}$ signal region, and non-combinatoric background, characterized by such peaking.

The combinatoric background arises from both continuum and $B \bar{B}$ events, and includes events in which pions or kaons are mis-identified, or in which there is a cross-over of tracks between the $B$ and $\bar{B}$ decays. The selection criteria described above in Sect. 3.2, and the Maximum Likelihood fitting procedure described below in Sect. 3.7, are designed to efficiently identify and reduce this kind of background.

Backgrounds which peak in the $m_{\mathrm{ES}}$ signal region include $B^{0}$ decays with the same $\pi \pi \pi K$ final state as our signal, $B^{0}$ decays in which there is a mis-identification of pions or kaons (since $m_{\mathrm{ES}}$ is independent of the particle type), and some $B^{ \pm}$decays in which there is a cross-over of tracks between the $B^{+}$and $B^{-}$. These peaking backgrounds are described in the following paragraphs. Additional selection criteria are also introduced based on mass vetoes to reduce the contributions of some of these channels.

Note that in the following, peaking background is defined to be events which appear in the $m_{\mathrm{ES}}$ signal region introduced in Sect. 3.4, irrespective of whether the 

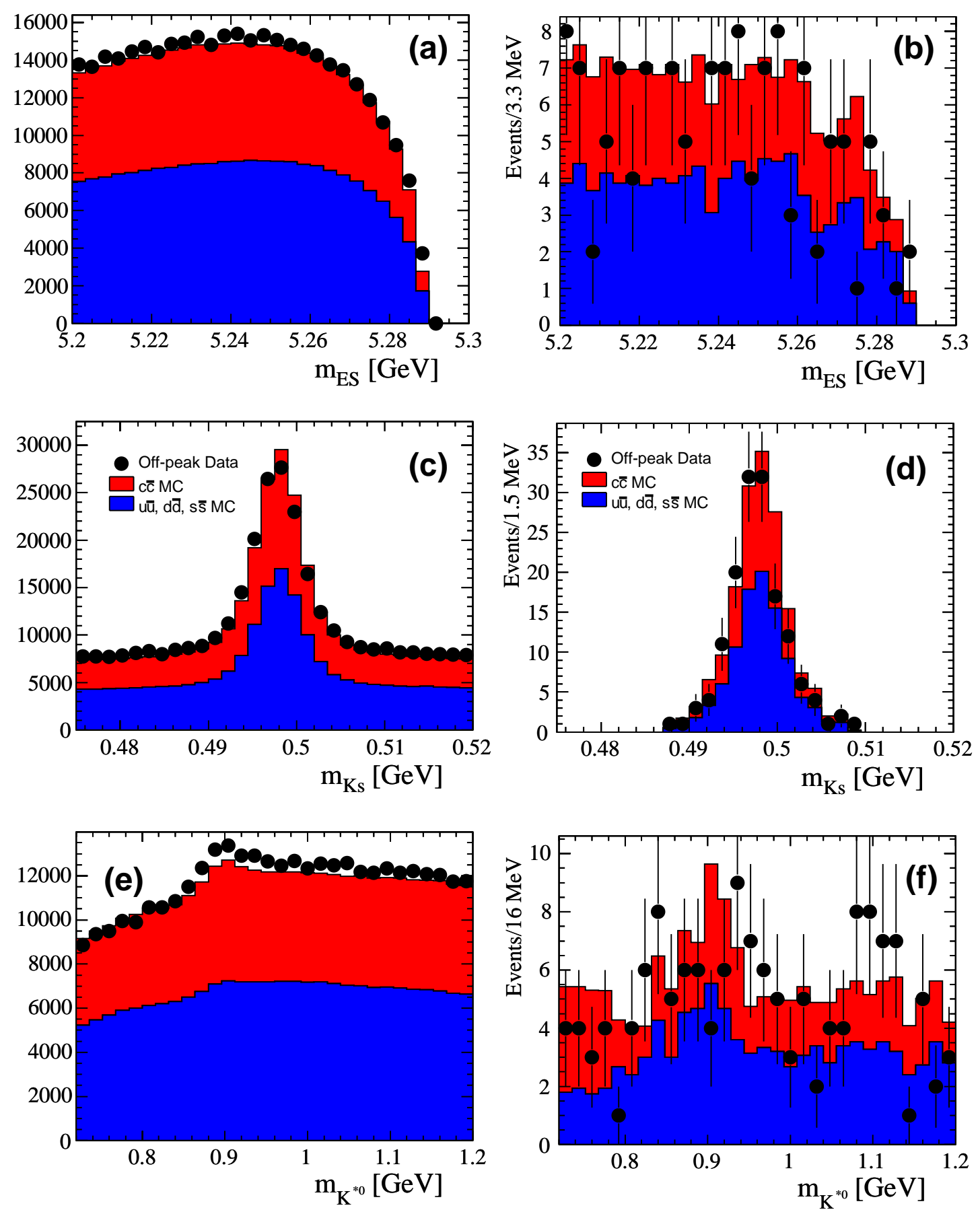

Figure 3.14: Comparison of MC with off-resonance data with (right) and without (left) selection criteria applied. The data points are the off-resonance data and the shaded histograms are the combined $q \bar{q} \mathrm{MC}$ samples. Plots (a) and (b) show the $m_{\mathrm{ES}}$ comparison without and with selection criteria applied respectively; Plots (c) and (d) show the $m_{K s}$ comparison; and Plots (e) and (f) show $m_{K * 0}$. 
channel actually exhibits a mass peak in that region. In contrast, combinatoric background is defined by events which pass the selection cuts of Sect. 3.2 but which do not appear within the $m_{\mathrm{ES}}$ signal region.

To identify sources of $B^{0} \bar{B}^{0}$ background with similar $m_{\mathrm{ES}}$ values to the signal, the $\mathrm{MC}$ truth information was examined for generic $B^{0} \bar{B}^{0} \mathrm{MC}$ events which satisfied the selection criteria of Sect. 3.2 and which, in addition, fell within the $m_{\mathrm{ES}}$ signal region defined in Sect. 3.4 (note that the signal region restrictions on $|\Delta E|$ and $m_{K^{* 0}}$ were not applied). Table 3.3 lists the decay modes identified in this manner, separated into four categories. In addition to the channels identified using this $\mathrm{MC}$ method, the $B^{0} \rightarrow K_{0}^{* 0}(1430) K_{S}^{0}$ channel was also studied, which is not in the MC but which is believed to be a potential source of background (see Sect. 3.6.2).

\subsection{1 $B^{0} \bar{B}^{0}$ with the same final state (Category 1)}

Category 1 background consists of $B^{0}$ decays with the same $\pi \pi \pi K$ final state as our signal. These modes are expected to peak in the signal regions of $m_{\mathrm{ES}}$ and $\Delta E$ but not in the signal region of $m_{K^{* 0}}$.

The first Category 1 channel, $B^{0} \rightarrow D^{\mp} K^{ \pm}$, arises from the false reconstruction of a $K^{* 0}$ from a combination of the $K^{ \pm}$with a $\pi^{\mp}$ from the decay $D^{\mp} \rightarrow \pi^{\mp} K_{S}^{0} \rightarrow$ $\pi^{\mp} \pi^{+} \pi^{-}$. The branching fraction of this channel is $(1.95 \pm 0.31) \times 10^{-6}[9]$. A veto on the $D^{\mp}$ mass was created using the invariant mass of the $K_{S}^{0}$ and the pion used to

reconstruct the $K^{* 0}$. This invariant mass is referred to below as $m_{K \pi}$. A Gaussian 
Table 3.3: $m_{\mathrm{ES}}$ peaking background modes in $B^{0}$ decays.

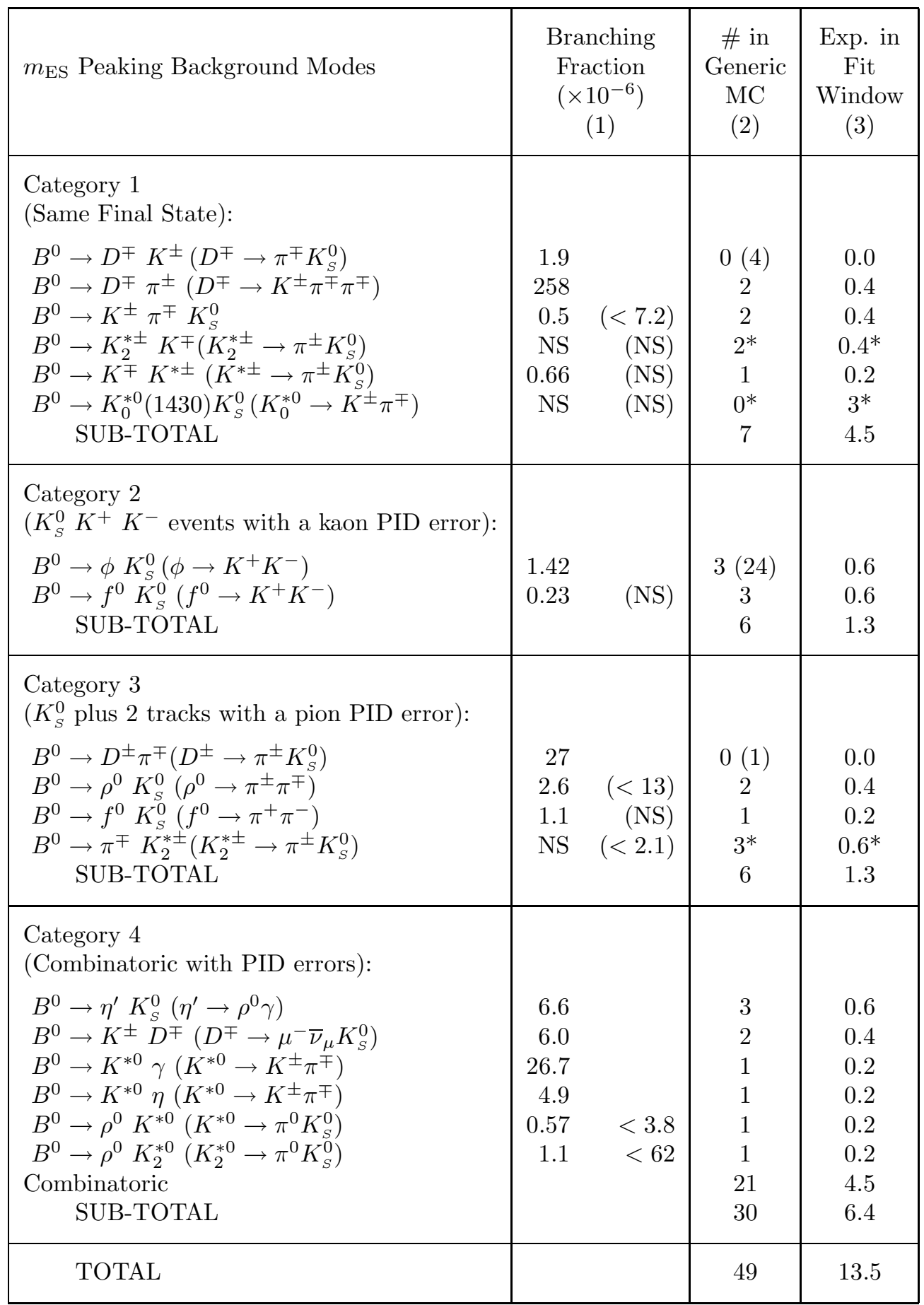

(1) Branching Fraction used in MC to the final state.

PDG value in parenthesis. NS $=$ Not Set

(2) The numbers in parenthesis are before the mass vetoes

* indicates BF was not set in MC.

(3) Column 2 times luminosity factor (208/976). 
fit to the $m_{K \pi}$ distribution of $5,000 D^{\mp} K^{ \pm}\left(D^{\mp} \rightarrow \pi^{\mp} K_{S}^{0}\right)$ MC events exhibited a peak at $m_{K \pi}=1.87 \mathrm{GeV} / c^{2}$ with a standard deviation of $\sigma_{K \pi}=8.0 \times 10^{-3} \mathrm{GeV} / c^{2}$. Due to large non-gaussian tails in the distribution, we required $m_{K \pi}$ to appear within $\pm 7 \sigma_{K \pi}$ of the nominal $D^{\mp}$ mass [9], corresponding to $1.813<m_{K \pi}<1.925 \mathrm{GeV} / c^{2}$. This cut removed $64 \%$ (883 of 1370 ) and $4.4 \%$ (49 of 1123), respectively, of the $K^{ \pm}$ $D^{\mp}$ background and signal $\mathrm{MC}$ events.

The second Category 1 channel, $B^{0} \rightarrow D^{\mp} \pi^{ \pm}$with $D^{\mp} \rightarrow K^{ \pm} \pi^{\mp} \pi^{\mp}$, arises from the mis-reconstruction of two pions into a $K_{S}^{0}$, and the kaon and a pion into a $K^{* 0}$. The branching fraction of this channel is $(2.64 \pm 0.09) \times 10^{-4}[9]$. A sample of 466,000 $D^{\mp} \pi^{ \pm}\left(D^{\mp} \rightarrow K^{ \pm} \pi^{\mp} \pi^{\mp}\right)$ MC events was used to study this source of background. An attempt was made to construct a veto on the $D^{\mp}$ mass based on the invariant mass of the $K^{* 0}$ candidate and the pion in the event with the same sign as the $D^{\mp}$. The resulting mass distribution was almost uniform, however. From an analysis of the MC truth information, it was determined that most of the $K_{S}^{0}$ candidates in these events were constructed using at least one track from a cross-over between the $B^{0}$ and $\bar{B}^{0}$. An effective veto for this mode was therefore not possible.

The third Category 1 channel, $B^{0} \rightarrow K^{ \pm} \pi^{\mp} K_{S}^{0}$, is a non-resonant three-body decay mode. The current upper limit on the branching ratio is $7.2 \times 10^{-6}[9]$. A sample of $1,193,000 K^{ \pm} \pi^{\mp} K_{S}^{0}$ MC events was used to determine the characteristics of this mode. Because of the similarity of these events to the signal, and because there is no intermediate resonance to serve as a potential veto, no distinguishing features 


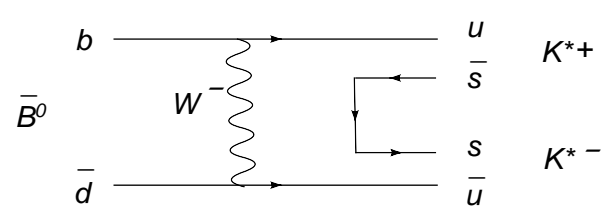

Figure 3.15: Virtual W exchange Feynman diagram.

were observed to allow the introduction of additional cuts.

Presently, there are no experimental results for the fourth and fifth channels listed

for Category 1: $B^{0} \rightarrow K_{2}^{* \pm} K^{\mp}\left(K_{2}^{* \pm} \rightarrow \pi^{ \pm} K_{S}^{0}\right)$ and $B^{0} \rightarrow K^{\mp} K^{* \pm}\left(K^{* \pm} \rightarrow \pi^{ \pm} K_{S}^{0}\right)$ (see Fig. 3.15. The experimental branching fractions of these channels are expected to be very small, however, because they are suppressed, first by the smallness of the CKM factor $V_{u b}$, second by the need to exchange a virtual $W$, and third by the need to create an $s \bar{s}$ pair. It is assumed that the contributions of these channels is overestimated in the MC and their event rates in the data is negligible.

\subsection{2 $B^{0} \rightarrow K_{0}^{* 0}(1430) K_{S}^{0}$ channel and other $S$-wave contribu- tions}

The last channel in Category 1 is $B^{0} \rightarrow K_{0}^{* 0}(1430) K_{S}^{0}$, with $K_{0}^{* 0}(1430) \rightarrow K^{+} \pi^{-}$. Currently, there are no experimental results for this channel and it is not present in the generic MC.

To study this mode, $10,000 B^{0} \rightarrow K_{0}^{* 0}(1430) K_{S}^{0}\left(K_{0}^{* 0}(1430) \rightarrow K^{+} \pi^{-}\right)$MC events were generated using EvtGen. After the selection criteria of Sect. 3.2 were applied, 
$1.4 \%$ (143 out of 10,000$)$ of these events remained.

The $K_{0}^{* 0}(1430)$ resonance cannot be considered separately from the total $S$-wave portion of the $K^{ \pm} \pi^{\mp}$ spectrum. It is possible that a $K^{ \pm} \pi^{\mp} S$-wave component of the $B$ decay amplitude contributes to $B^{0} \rightarrow K^{+} \pi^{-} K_{S}^{0}$ in the region of $m_{K \pi}$ included in our Maximum Likelihood fit. This $K^{ \pm} \pi^{\mp} S$-wave amplitude could correspond to the lower tail of the $K^{* 0}(1430)$, a non-resonant three-body channel, or both. A previous BABAR Dalitz analysis of $B^{ \pm} \rightarrow K^{ \pm} \pi^{\mp} \pi^{ \pm}[36]$ included a $K^{ \pm} \pi^{\mp} S$-wave amplitude of the form used by the LASS Collaboration [37, 38], which is a combination of the $K^{* 0}(1430)$ and a non-resonant component. This analysis found that a substantial fraction of the $K^{+} \pi^{-} \pi^{+}$final state goes through the $K^{* 0}(1430) \pi^{+}$channel, with a branching fraction roughly four times larger than that of $K^{* 0}(890) \pi^{+}$. This was also seen by the Belle Collaboration, in a Dalitz analysis of $B^{ \pm} \rightarrow K^{ \pm} \pi^{\mp} \pi^{ \pm}[39]$.

The $K^{* 0}(890)$ and an $S$-wave $K \pi$ amplitude (e.g. the $\left.K^{* 0}(1430)\right)$ will quantum mechanically interfere with an unknown relative phase. If the the two amplitudes are $A_{1}$ and $A_{2}$ and the relative phase difference is $\delta$, the combined probability, $|A|^{2}$, is give by eq 3.6

$$
\begin{aligned}
|A|^{2} & =\left|A_{1}+A_{2} e^{i \delta}\right|^{2} \\
& =\left|A_{1}\right|^{2}+\left|A_{2}\right|^{2}+2 \mathcal{R} e\left(A_{1} A_{2}^{*}\right) \cos \delta+2 \mathcal{I} m\left(A_{1} A_{2}^{*}\right) \sin \delta
\end{aligned}
$$

where the fact that $A_{1} A_{2}^{*}+A_{1}^{*} A_{2}=2 \mathcal{R} e\left(A_{1} A_{2}^{*}\right)$ and $A_{1} A_{2}^{*}-A_{1}^{*} A_{2}=2 i\left[\mathcal{I} m\left(A_{1} A_{2}^{*}\right)\right]$ has been used. If the spin states are explicitly taken into account and not summed 
or averaged, the resonance amplitudes are proportional to Legendre Polynomials of the cosine of the helicity angle, $\cos \theta_{H}[29,30]$

$$
A_{x} \propto P_{l_{x}}^{m_{x}}\left(\cos \theta_{H}\right)
$$

where $m_{x}$ and $l_{x}$ are the spin and orbital angular momentum quantum numbers for the particle. The orthogonality of the Legendre polynomials ensures that for resonances with different spins the effect of the interference will integrate to zero provided our selection and reconstruction efficiency is symmetric in the cosine of the $K \pi$ helicity angle, $\cos \theta_{H}$.

To confirm this symmetry, the selection efficiency was evaluated over four regions of $m_{K \pi}$ near the resonance peak:

$$
\begin{aligned}
& 0.82 \leq m_{K \pi}<0.86 \\
& 0.86 \leq m_{K \pi}<0.90 \\
& 0.90 \leq m_{K \pi}<0.94 \\
& 0.94 \leq m_{K \pi}<0.98 .
\end{aligned}
$$

Figures 3.16 (a) through $(\mathrm{d})$ show the $\operatorname{Cos}\left(\theta_{\text {Helicity }}\right)$ distribution for each region of $m_{K \pi}$. The black histograms represent the generated MC truth values and the red (less populated) histograms represent the reconstructed MC after all cuts have been applied. Figs. 3.16 (e) through (h) show the efficiency distributions for these regions 
of $m_{K \pi}$, defined by the ratios of the red to the black histograms in fig 3.16(a)(d). The mean efficiency for positive and negative $\operatorname{Cos}\left(\theta_{\text {Helicity }}\right)$ are also shown on Figures 3.16(e) - 3.16(h).

The differences between positive and negative $\operatorname{Cos}\left(\theta_{\text {Helicity }}\right)$ are between $4 \%$ and $17 \%$. To better understand these differences, the effects of the mass vetoes (See Sections 3.6.1 and 3.6.3) were considered (Sect. 3.6) on the $\operatorname{Cos}\left(\theta_{\text {Helicity }}\right)$ distributions. Fig. 3.17(a) shows the distribution of the $\phi$ invariant mass used to veto $\phi K_{S}$ events versus $\operatorname{Cos}\left(\theta_{\text {Helicity }}\right)$. The limits of the mass veto are shown on the figure by the two horizontal lines. Fig. 3.17(b) shows the distribution of the $D K$ invariant mass used to veto $D K$ events versus $\operatorname{Cos}\left(\theta_{\text {Helicity }}\right)$. The horizontal lines on the figure indicate the limits of the mass veto applied.

The effect of the $\phi$ mass veto is to slightly reduce the number of events with negative $\operatorname{Cos}\left(\theta_{\text {Helicity }}\right)$. The effect of the $D K$ veto is to create a narrow deficit in the $\operatorname{Cos}\left(\theta_{\text {Helicity }}\right)$ distribution near $\operatorname{Cos}\left(\theta_{\text {Helicity }}\right)=0.75$. This mass veto accounts for the drop in efficiency around $\operatorname{Cos}\left(\theta_{\text {Helicity }}\right)=0.75$ in figs. 3.17(e)-(h).

The asymmetry in the selection efficiency is not believed to be significant and therefore the $S-P$ interference effects will average out in the $m_{K \pi}$ projection, which is used in the Maximum Likelihood fit. This means that the $K^{* 0}(890)$ and the $S$ wave contributions can be treated as separate, independent fit components (PDFs) in the analysis and that the $K^{* 0}(890) K_{S}^{0}$ branching fraction calculated from the fitted $K^{* 0}(890)$ yield needs no correction for $S-P$ interference effects. 

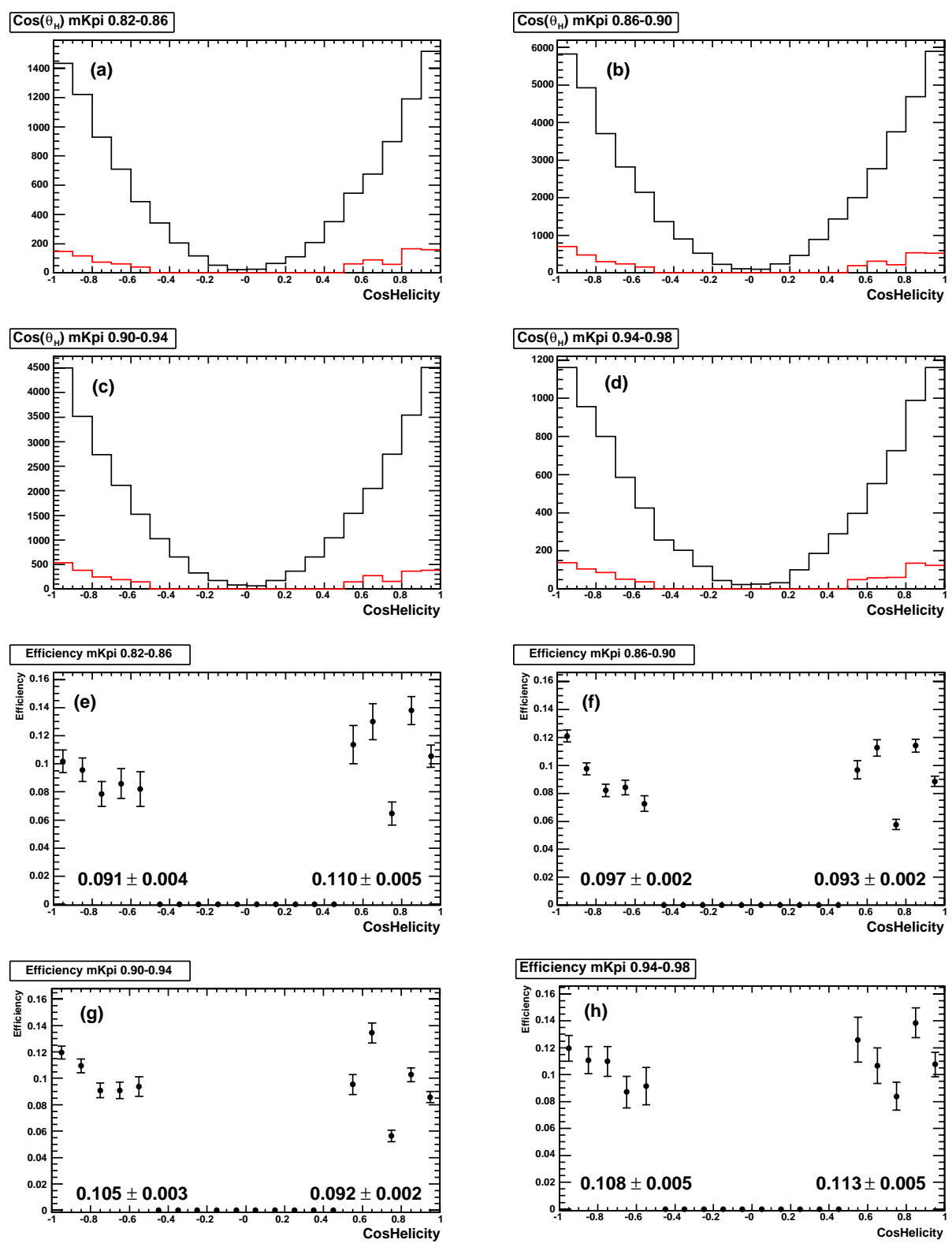

Figure 3.16: Efficiency vs. $\operatorname{Cos}\left(\theta_{\text {Helicity }}\right)$. Plots (a) through (d) show the distribution of the $\operatorname{Cos}\left(\theta_{\text {Helicity }}\right)$ for four different regions of $m_{K \pi}$. The black histograms represent the generated MC truth values and the red histograms represent the reconstructed MC after all cuts have been applied. Plots (e) through (h) show the efficiency distributions for these regions of $m_{K \pi}$. 


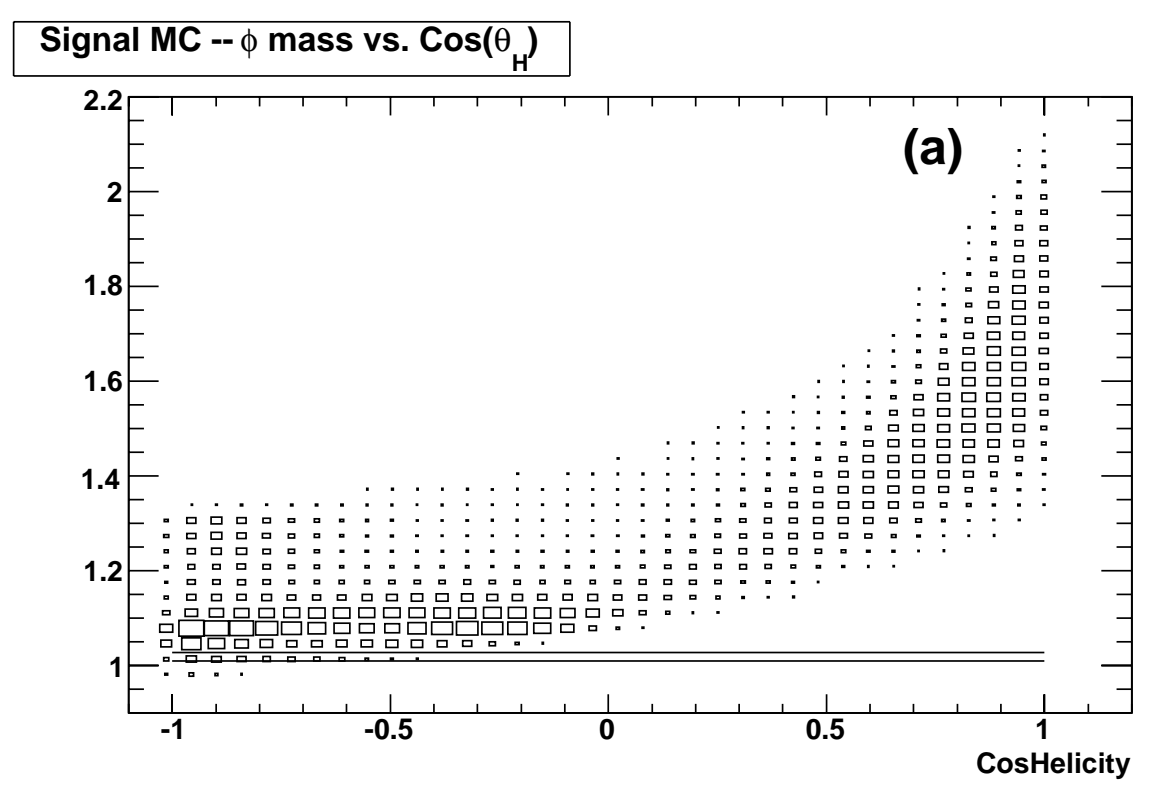

Signal MC -- DK mass vs. $\operatorname{Cos}\left(\theta_{H}\right)$

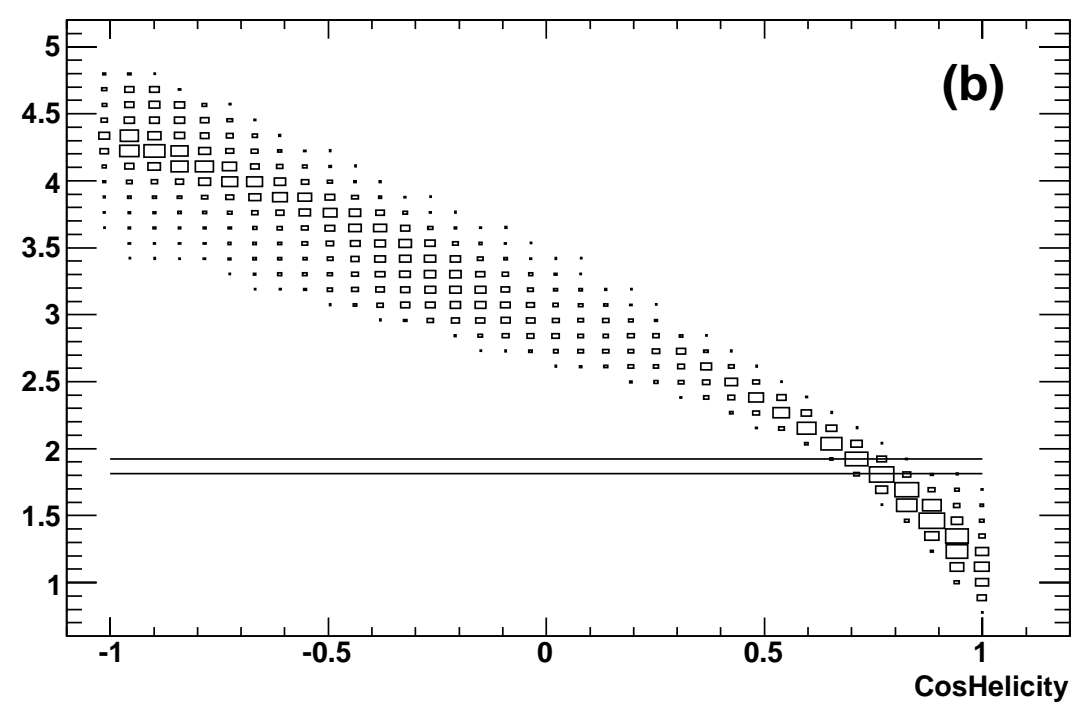

Figure 3.17: Mass vetoes vs. $\operatorname{Cos}\left(\theta_{\text {Helicity }}\right)$. Plot (a) shows the distribution of the $\phi$ invariant mass used to veto $\phi K_{S}$ events versus $\operatorname{Cos}\left(\theta_{\text {Helicity }}\right)$ and the range of the veto (horizontal lines). Plot (b) shows the distribution of the $D K$ invariant mass used to veto $D K$ events versus $\operatorname{Cos}\left(\theta_{\text {Helicity }}\right)$ and the range of the veto (horizontal lines). 
A very low number of $B^{0} \rightarrow K^{+} \pi^{-} K_{S}^{0}$ events is anticipated, regardless of the sub-channel $\left(K^{* 0}(890) K_{S}^{0}\right.$ or otherwise). The only discriminating variable that can be used to distinguish $K^{* 0}(890) K_{S}^{0}$ from $K^{* 0}(1430) K_{S}^{0}$ or non-resonant $K^{+} \pi^{-} K_{S}^{0}$ events is $m_{K \pi}$. It was possible to simultaneously fit for the $K^{* 0}(890) K_{S}^{0}$ yield and a $(K \pi)_{S} K_{S}^{0}$ yield, and therefore this yield has been included in the ML fit.

\subsection{3 $B^{0} \bar{B}^{0}$ with a $K_{S}^{0}$ and a Kaon PID Error (Category 2)}

Category 2 background (Table 3.3 ) consists of $B^{0}$ decays to a $K_{S}^{0} K^{+} K^{-}$final state, where a PID error is made for one of the charged Kaon's such that it is misidentified as a pion. This category of background is expected to peak in the $m_{\mathrm{ES}}$ signal region, but to exhibit a peak in $\Delta E$ that is negatively displaced from the signal region value centered on zero.

The first mode in this category (see Table 3.3) is $B^{0} \rightarrow \phi K^{0}$. After imposing the cuts described in Sect. 3.2, this channel was found to contribute a significant number of events to the $m_{\mathrm{ES}}$ signal region (Table 3.3). To reduce this background, a veto on the $\phi$ meson mass was developed, assuming the pion candidate used to reconstruct the $K^{* 0}$ to be a kaon. The invariant mass of the two charged kaons is referred to below as $m_{K K}$. A Gaussian fit of $5,000 B^{0} \rightarrow \phi K_{S}^{0}\left(K_{S}^{0} \rightarrow \pi^{+} \pi^{-}\right)$ MC events exhibited a peak at $m_{K K}=1.021 \mathrm{GeV} / c^{2}$, with a standard deviation of $\sigma_{K K}=3.7 \times 10^{-3} \mathrm{GeV} / c^{2}$. The $\phi$ veto required that $m_{K K}$ appear within $\pm 2.5 \sigma_{K K}$ of the nominal $\phi$ mass [9], i.e. it was required that $1.0098<m_{K K}<1.028 \mathrm{GeV} / c^{2}$. 
This veto eliminated $87 \%$ (1274 of 1467) of the $\phi K^{0}$ background MC events but only $1.2 \%$ (13 of 1123) of the signal MC events.

No attempt was made to develop a veto for the other category 2 channel, $B^{0} \rightarrow$ $f^{0} K_{S}^{0}\left(f^{0} \rightarrow K^{+} K^{-}\right)$, because of the small number of events in this channel and the relatively broad width of the $f^{0}$. Instead, this channel is accounted for by implementing a separate probability density function for Category 2 events in the Maximum Likelihood fit (see Sect. 3.7).

\subsection{4 $B^{0} \bar{B}^{0}$ with a $K_{S}^{0}$ and a Pion PID Error (Category 3)}

Category 3 events consist of $B^{0}$ decays to $K_{S}^{0} \pi^{+} \pi^{-}$, where one of the pions is misidentified as a charged kaon. This category of background peaks in the $m_{\mathrm{ES}}$ signal region but exhibits a peak in $\Delta E$ that is positively displaced from the signal region centered on zero.

The four Category 3 decay modes are listed in Table 3.3. None of them make a large contribution to the overall background. They are accounted for by implementing a separate probability density function for Category 3 events in the Maximum Likelihood fit.

\subsection{5 $B^{0} \bar{B}^{0}$ Other (Category 4)}

Category 4 contains all peaking $B^{0} \bar{B}^{0}$ background events not included in Categories 1, 2 and 3. The decay channels so identified are listed in Table 3.3. Category 4 
also includes $B^{+} B^{-}$decays which pass the selection cuts and peak in $m_{\mathrm{ES}}$ (see the following section) as well as the combinatoric components of the $B^{0} \bar{B}^{0}$ and $B^{+} B^{-}$ samples.

Category 4 decay modes are not expected to peak in $\Delta E$. Category 4 channels are accounted for by implementing a separate probability density function for Category 4 events in the Maximum Likelihood fit.

\subsection{6 $\quad B^{+} B^{-}$-Related Background}

Similar to $B^{0} \bar{B}^{0} \mathrm{MC}$ events, $B^{+} B^{-} \mathrm{MC}$ events, which fell into the $m_{\mathrm{ES}}$ signal region, were examined after the selection criteria of Sect. 3.2 had been applied. Of the 33 events so identified, six arose from the decay $B^{0} \rightarrow D^{0} K^{ \pm}$followed by $D^{0} \rightarrow K^{0} \pi^{0}$, in which there was a high multiplicity $B^{\mp}$ decay on the other side of the event. The background arose because a $\pi^{ \pm}$from the other side of the event was substituted for the $\pi^{0}$. Of the 33 events, five other events were similar in that a $\pi^{ \pm}$from the other side was substituted for a $\pi^{0}$. The remaining events appear to be random combinations of tracks.

The $B^{+} B^{-}$background distributions of $m_{\mathrm{ES}}, \Delta E$, and $m_{K^{* 0}}$ are essentially identical to those of the Category 4 portion of the $B^{0} \bar{B}^{0}$ background. Therefore, for simplicity, $B^{+} B^{-}$background events are included in Category 4 as mentioned in Sect. 3.6.5. 


\subsection{The Extended Maximum Likelihood Fit}

An unbinned extended Maximum Likelihood fit was used to determine the number of events in the signal $\left(N^{s i g}\right)$, continuum background $\left(N^{\text {cont }}\right)$, and five categories of $B \bar{B}$ background $\left(N^{\text {Bcat } 1}, N^{S-\text { wave }}, N^{\text {Bcat2 }}, N^{\text {Bcat3 }}\right.$, and $\left.N^{\text {Bcat } 4}\right)$, with $m_{\mathrm{ES}}, \Delta E$, and $m_{K^{* 0}}$ the fitted experimental distributions. The likelihood, $\mathcal{L}$, for obtaining the selected sample is given by the product of the probabilities for the individual events:

$$
\mathcal{L}=\frac{e^{-\nu}}{n !} \prod_{i=1}^{n}\left(\sum_{Q} N^{Q} \mathcal{P}_{i}^{Q}\right)
$$

where $\nu=\sum N^{Q}, n$ is the total number of events, $N^{Q}$ is the yield, $\mathcal{P}_{i}^{Q}$ is the probability evaluated for event $i$, and $Q$ represents signal, continuum background, or one of the five $B \bar{B}$ background categories. The RooFit package [40] was used to minimize the quantity $-2 \log \mathcal{L}$, which changes the above product into a sum and is equivalent to maximizing $\mathcal{L}$. The quantity $-2 \log \mathcal{L}$ is referred to as the Negative Log Likelihood (NLL).

The probabilities $\mathcal{P}_{i}^{Q}$ are themselves products of three Probability Density Functions (PDFs), evaluated for event $i$ :

$$
\mathcal{P}_{i}^{Q}=\left(\mathcal{P}_{m e s}^{Q} \mathcal{P}_{\Delta E}^{Q} \mathcal{P}_{m K^{*}}^{Q}\right)_{i}
$$

The analytical parameterizations used for the PDFs are presented below in Sect. 3.7.2. 


\subsubsection{Correlations Between Fitted Variables}

The Maximum Likelihood methodology depends on the independence of the variables in the fit. The degree of independence between variables was evaluated using the correlation coefficient, $\rho$, defined by

$$
\rho \equiv \frac{\text { Covariance }(x, y)}{\sigma_{x} \sigma_{y}}
$$

where $x$ and $y$ represent $m_{\mathrm{ES}}, \Delta E$, or $m_{K^{* 0}}$. The covariance (cov) of two variables is defined as

$$
\operatorname{cov}(x, y) \equiv\langle x y\rangle-\langle x\rangle\langle y\rangle
$$

and $\sigma_{x} \equiv \sqrt{\left\langle x^{2}\right\rangle-\langle x\rangle^{2}}$. The correlations were examined independently using both signal and background MC events. The background events were divided into the different samples described in sec. 3.6 and Table 3.3.

The fraction of each decay channel in a particular sample was determined by the product of the branching fraction and cut efficiency for that channel. Scatter plots of the three combinations of variables are shown in Figs. 3.18 - 3.23. The corresponding correlation coefficients are presented in Table 3.4. For signal events, the correlation coefficients are all less than 15\%. Several of the correlations in background events are as large as about $30 \%$, however. To test the sensitivity of the correlations to outlying events (defined by events with $m_{\mathrm{ES}}<5.270 \mathrm{GeV} / c^{2}$ ), we removed these events and re-calculated the coefficients. The results are indicated in Table 3.4 by the rows 
Table 3.4: Correlation coefficients between the three variables used in our fit. "tight" indicates a cut on $m_{\mathrm{ES}}>5.270$ was imposed.

\begin{tabular}{|l|r|r|r|r|}
\hline MC Sample & Sample Size & $\Delta E-m_{\mathrm{ES}}$ & $m_{K^{* 0}}-\Delta E$ & $m_{K^{* 0}-}-m_{\mathrm{ES}}$ \\
\hline Signal MC & 1,060 & -0.148 & 0.041 & -0.103 \\
Signal MC-tight & 1,024 & -0.137 & 0.049 & 0.031 \\
B bkg Category 1 MC & 620 & 0.057 & -0.062 & -0.002 \\
B bkg Category 2 MC & 284 & -0.330 & 0.229 & -0.019 \\
B bkg Category 2-tight & 277 & -0.064 & 0.221 & 0.030 \\
B bkg Category 3 MC & 531 & 0.193 & -0.050 & -0.156 \\
B bkg Category 3-tight & 456 & -0.074 & -0.032 & 0.024 \\
B bkg Category 4 MC & 217 & 0.042 & 0.046 & -0.052 \\
Continuum MC & 1,003 & 0.032 & -0.005 & 0.010 \\
\hline
\end{tabular}

labeled "tight." After eliminating outlying events, all correlation coefficients are seen to be less than about $10 \%$, with the exception of the $\Delta E-m_{\mathrm{ES}}$ correlation for signal MC $(13.7 \%)$ and the $m_{K^{* 0}-\Delta E}$ correlation for B background category $2 \mathrm{MC}(22.1 \%)$. The possible effects of these residual correlations are addressed in Section 3.8 and are incorporated into the systematic uncertainties.

\subsubsection{Probability Density Functions}

In this section, the Probability Density Functions (PDFs) $\mathcal{P}_{m e s}^{Q}, \mathcal{P}_{\Delta E}^{Q}$ and $\mathcal{P}_{m K^{*}}^{Q}$ are described (see Eq. (3.9)).

Various trial PDFs were suggested by previous studies of $B \bar{B}$ events and their backgrounds: a Gaussian distribution, a bifurcated Gaussian distribution (i.e. an 

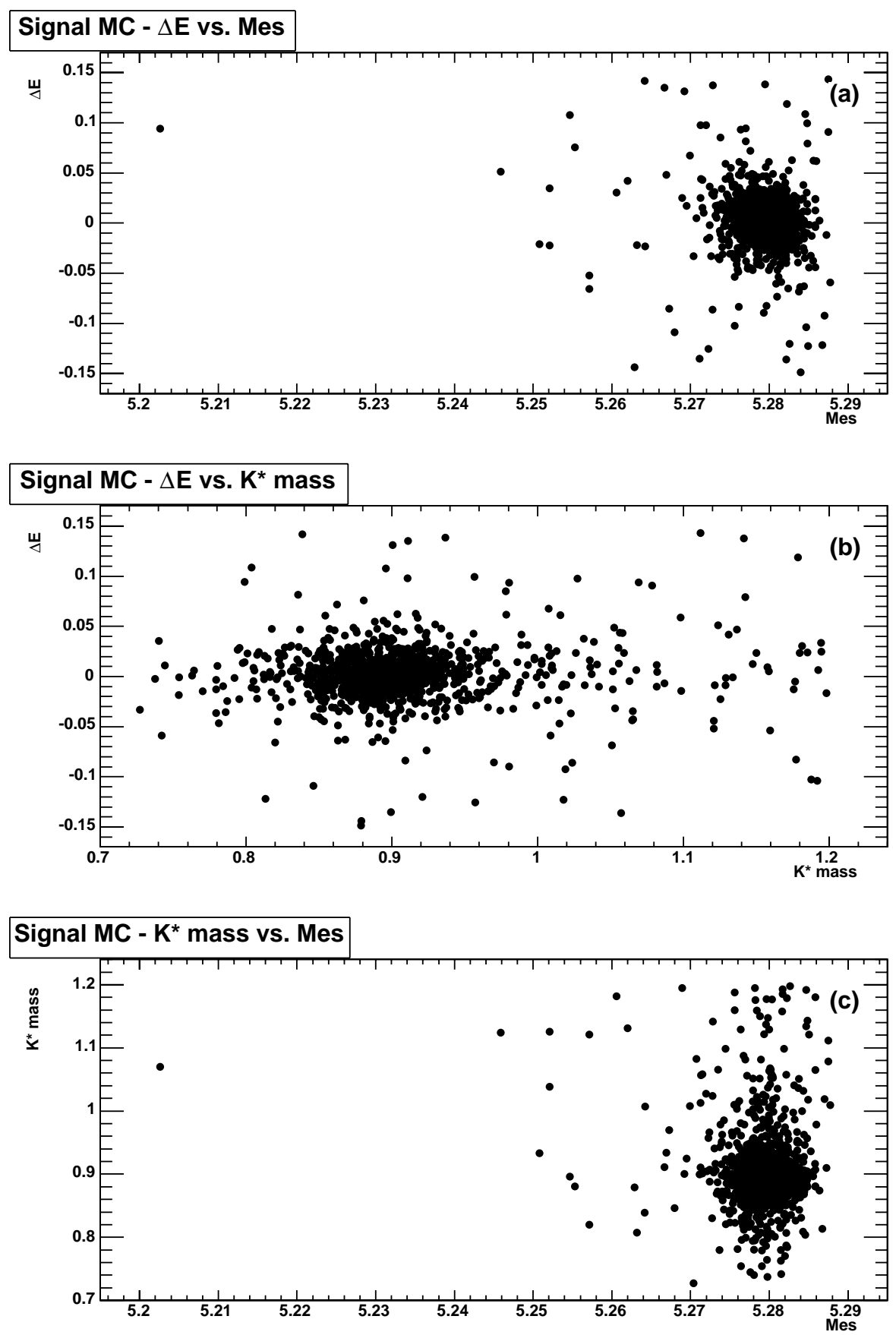

Figure 3.18: Signal MC scatter plots of $\Delta E$ vs. $m_{\mathrm{ES}}$ (a), $\Delta E$ vs. the invariant mass of the $K^{* 0}(\mathrm{~b})$, and the $K^{* 0}$ mass vs. $m_{\mathrm{ES}}(\mathrm{c})$. 
B Bkg Category 1 - $\Delta$ E vs. Mes

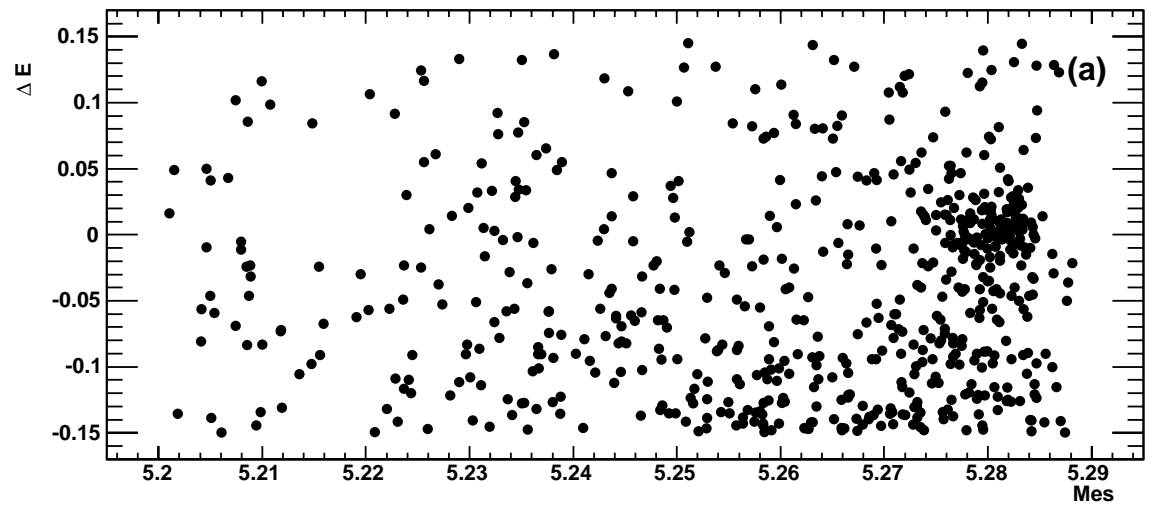

B Bkg Category $1-\Delta$ E vs. $K^{\star}$ mass

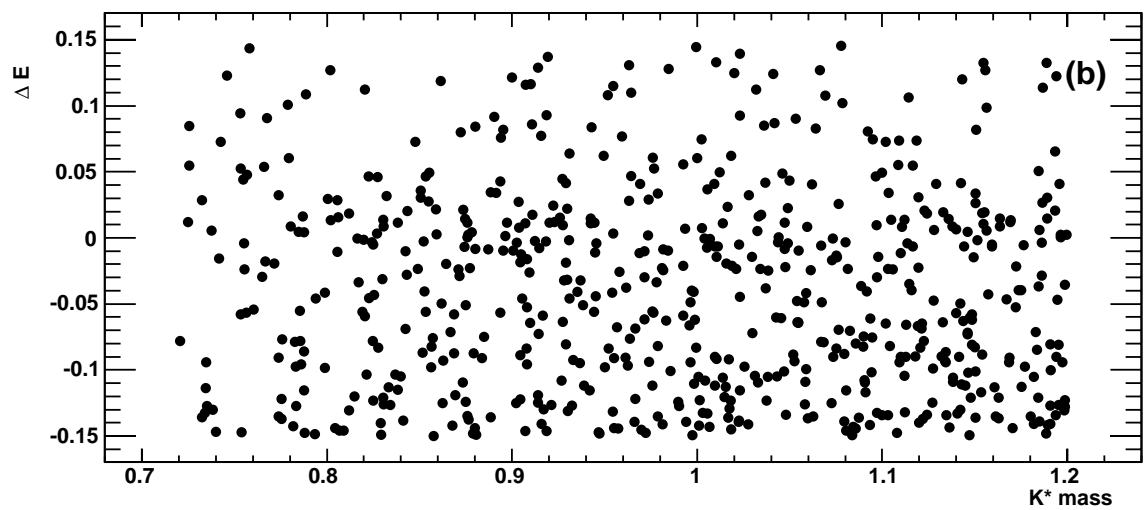

B Bkg Category 1 - $\mathrm{K}^{\star}$ mass vs. Mes

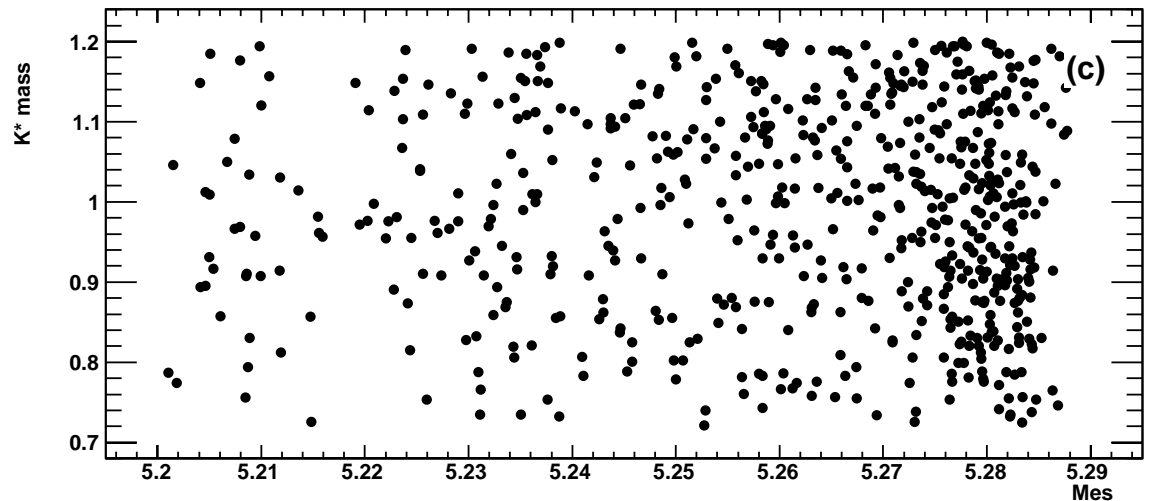

Figure 3.19: Category $1 B^{0} \bar{B}^{0}$ background MC scatter plots of $\Delta E$ vs. $m_{\mathrm{ES}}$ (a), $\Delta E$ vs. the invariant mass of the $K^{* 0}(\mathrm{~b})$, and the $K^{* 0}$ mass vs. $m_{\mathrm{ES}}(\mathrm{c})$. 

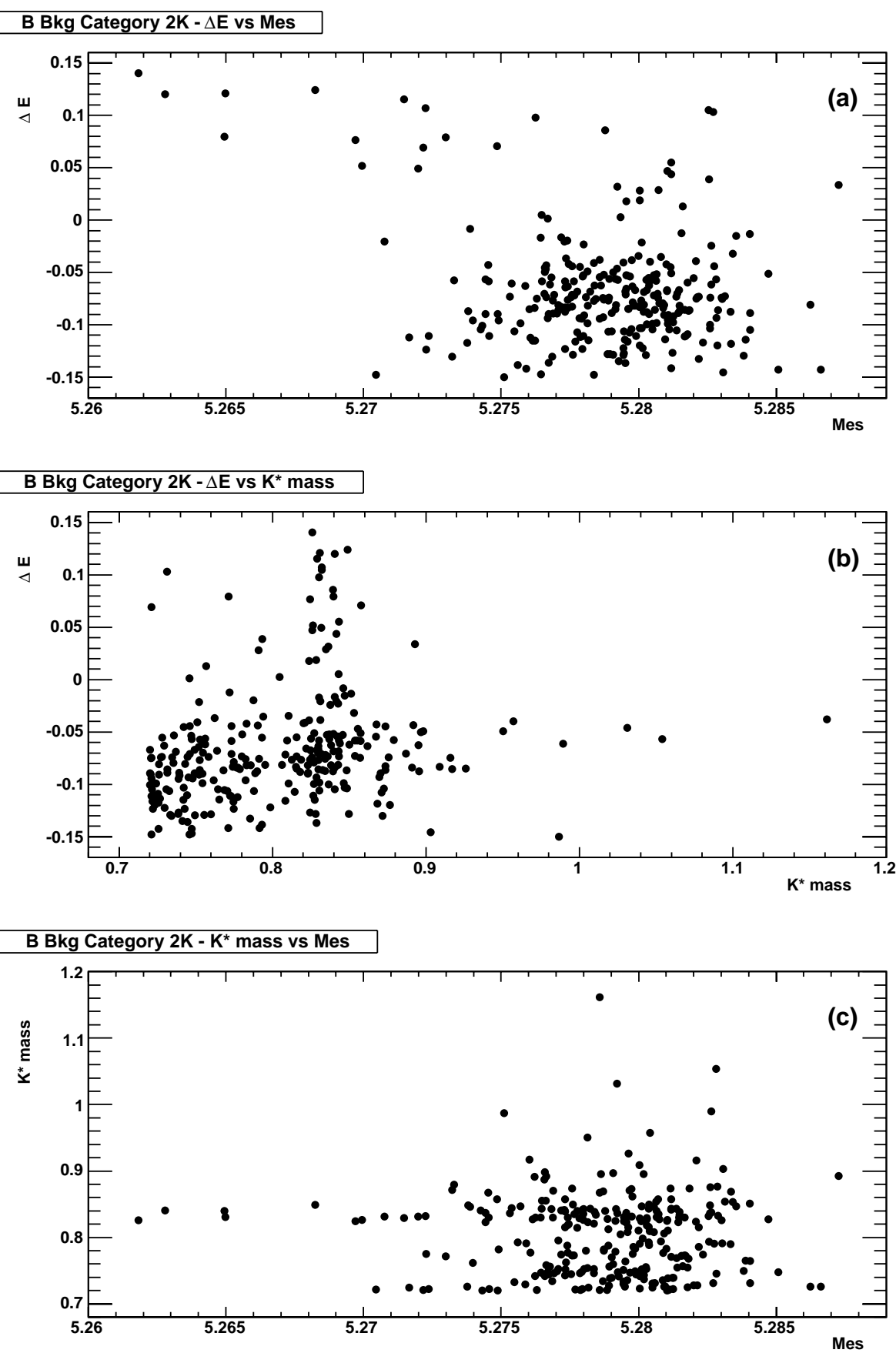

Figure 3.20: Category $2 B^{0} \bar{B}^{0}$ background MC scatter plots of $\Delta E$ vs. $m_{\mathrm{ES}}$ (a), $\Delta E$ vs. the invariant mass of the $K^{* 0}(\mathrm{~b})$, and the $K^{* 0}$ mass vs. $m_{\mathrm{ES}}(\mathrm{c})$. 
B Bkg Category 2Pi - $\Delta \mathrm{E}$ vs Mes

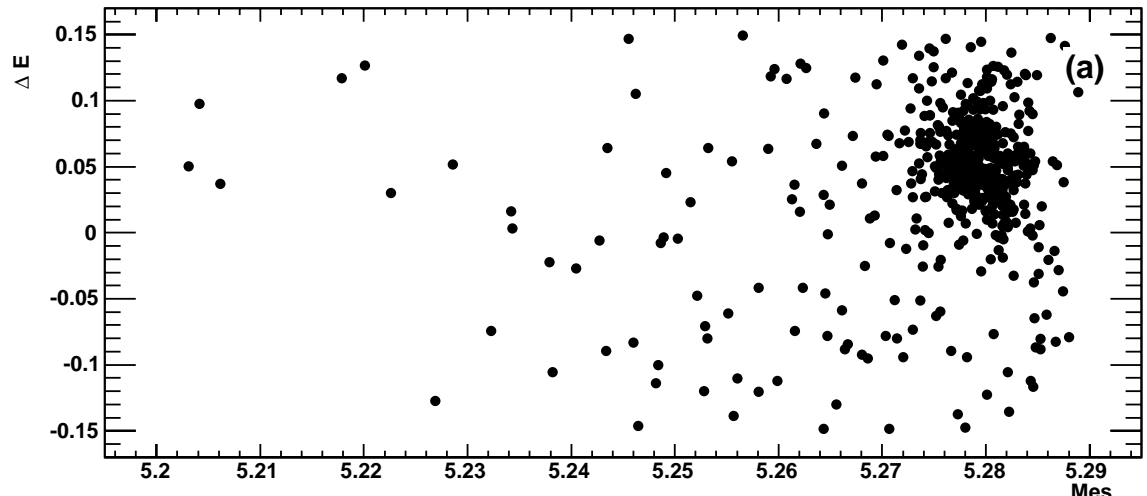

B Bkg Category $2 \mathrm{Pi}-\Delta \mathrm{E}$ vs $\mathrm{K}^{\star}$ mass

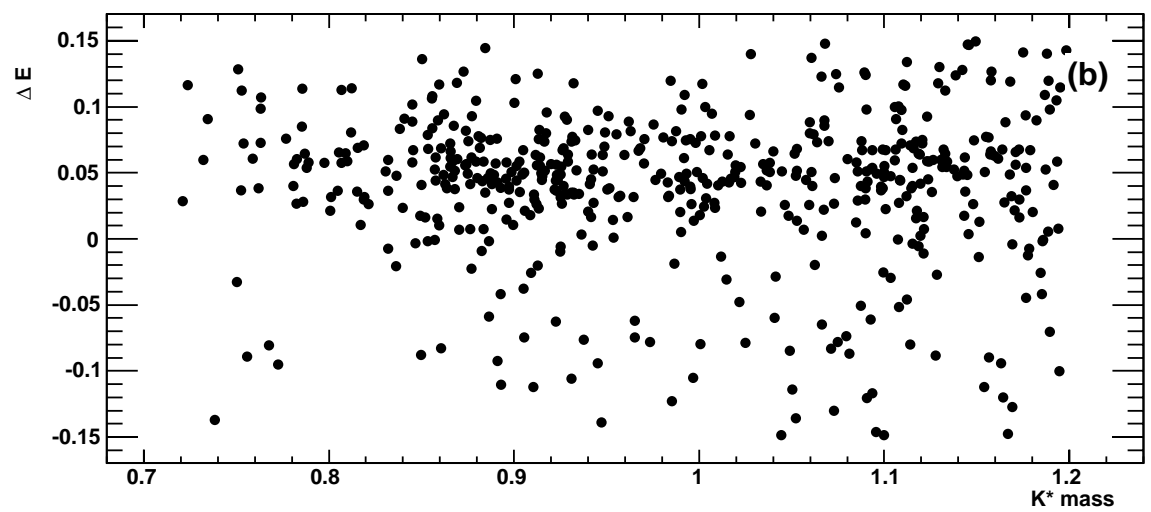

B Bkg Category $2 \mathrm{Pi}-\mathrm{K}^{\star}$ mass vs Mes

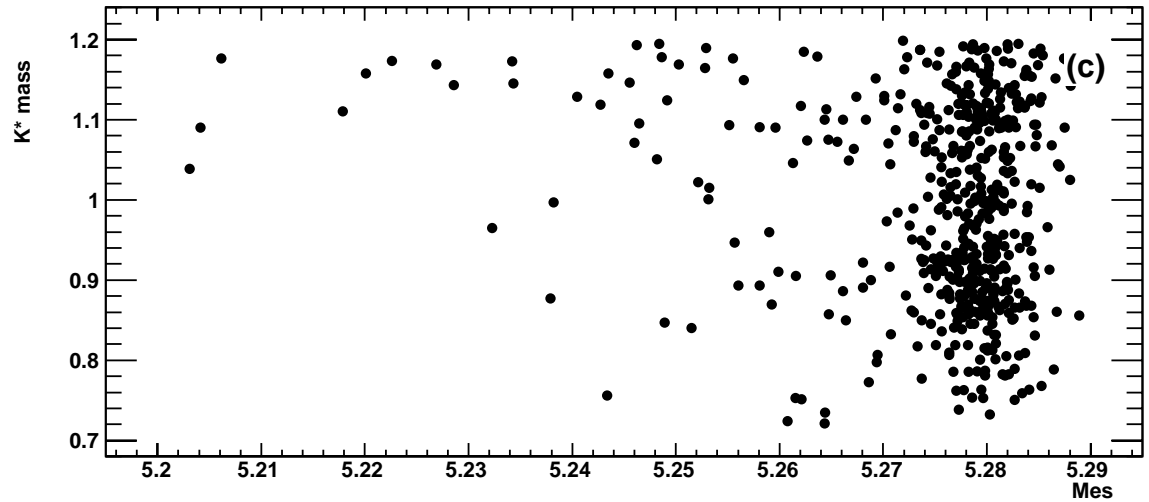

Figure 3.21: Category $3 B^{0} \bar{B}^{0}$ background MC scatter plots of $\Delta E$ vs. $m_{\mathrm{ES}}$ (a), $\Delta E$ vs. the invariant mass of the $K^{* 0}(\mathrm{~b})$, and the $K^{* 0}$ mass vs. $m_{\mathrm{ES}}(\mathrm{c})$. 

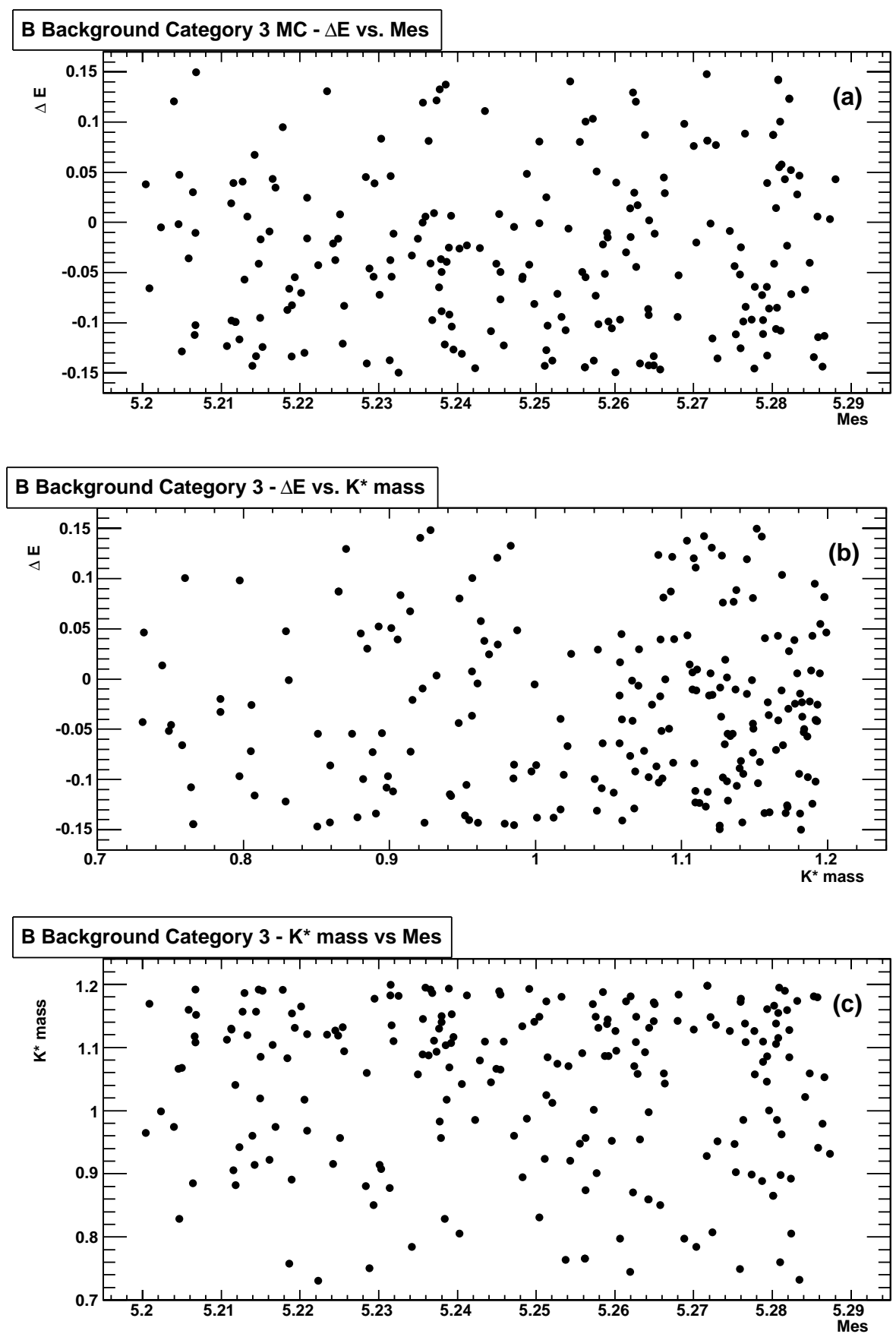

Figure 3.22: $B \bar{B}$ background Category $4 \mathrm{MC}$ scatter plots of $\Delta E$ vs. $m_{\mathrm{ES}}$ (a), $\Delta E$ vs. the invariant mass of the $K^{* 0}(\mathrm{~b})$, and the $K^{* 0}$ mass vs. $m_{\mathrm{ES}}(\mathrm{c})$. 

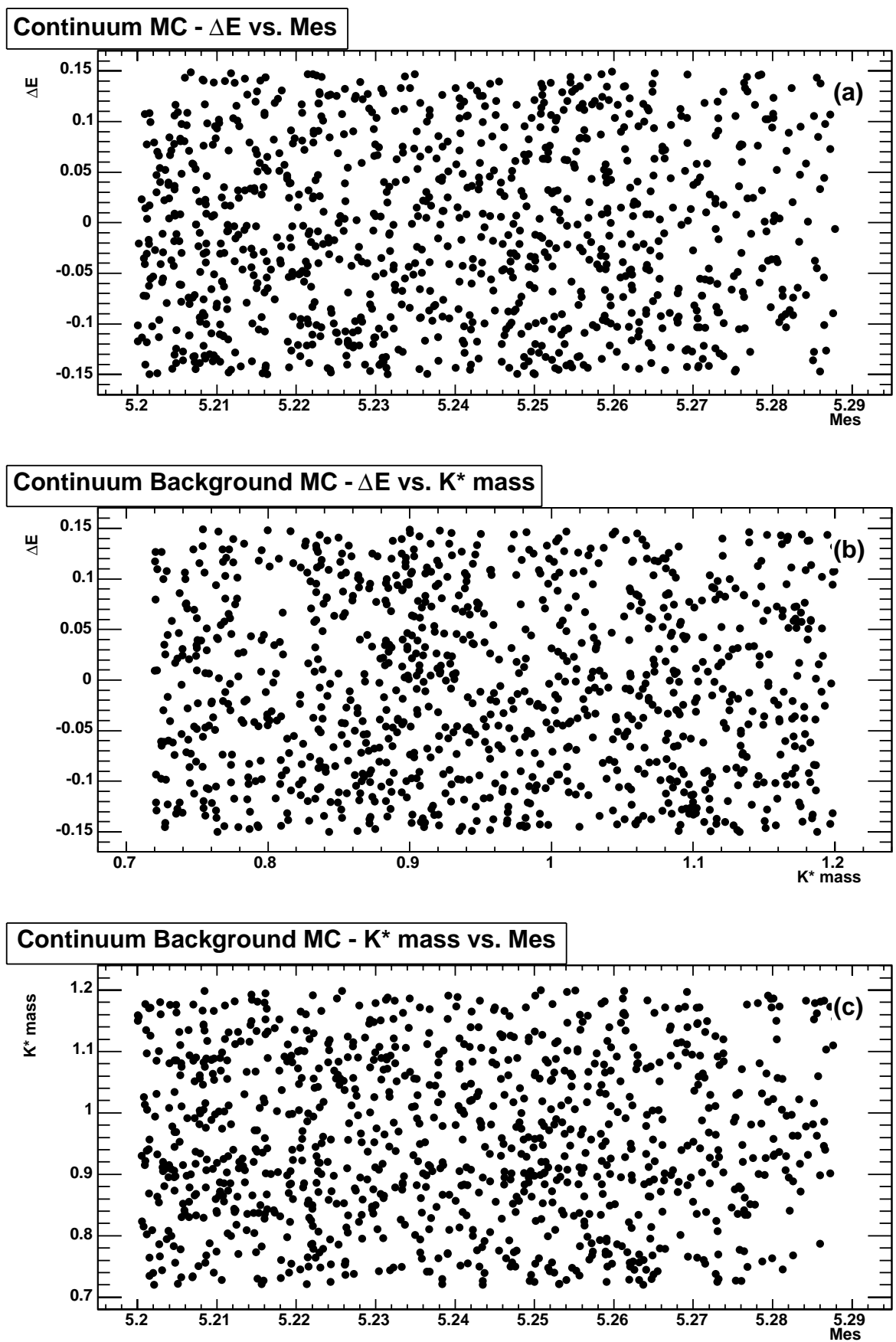

Figure 3.23: Continuum background MC scatter plots of $\Delta E$ vs. $m_{\mathrm{ES}}$ (a), $\Delta E$ vs. the invariant mass of the $K^{* 0}(\mathrm{~b})$, and the $K^{* 0}$ mass vs. $m_{\mathrm{ES}}(\mathrm{c})$. 
asymmetric distribution with the left side described by a Gaussian with standard deviation $\sigma_{1}$, and the right side by a Gaussian with standard deviation $\sigma_{2} \neq \sigma_{1}$ ), a polynomial distribution, the so-called Crystal Ball function [33],

$$
\begin{aligned}
\frac{\mathrm{d} N}{\mathrm{~d} x} & =\frac{A(n / \alpha)^{n} \exp \left(-\alpha^{2} / 2\right)}{\left[(\mu-x) /\left(\sigma+\frac{n}{\alpha}-\alpha\right)\right]^{n}} \quad ; \quad x \leq \mu-\alpha \sigma \\
& =A \exp \left[-\frac{(x-\mu)^{2}}{2 \sigma^{2}}\right] \quad ; \quad x>\mu-\alpha \sigma
\end{aligned}
$$

the so-called ARGUS function [34],

$$
\frac{\mathrm{d} N}{\mathrm{~d} x}=A x \sqrt{1-\left(x / x_{\text {max. }}\right)^{2}} \exp \left[-\xi\left(1-\left(x / x_{\text {max. }}\right)^{2}\right)\right]
$$

and the Breit-Wigner function

$$
\frac{\mathrm{d} N}{\mathrm{~d} x}=\frac{A}{(x-\mu)^{2}+(\Gamma / 2)^{2}} .
$$

The Crystal Ball function is a Gaussian with an exponential tail, with $\alpha, n, \sigma$ and $\mu$ as fitted parameters. The ARGUS function has two parameters, the so-called ARGUS parameter $\xi$ and the cutoff $x_{\max }$. The two parameters of the Breit-Wigner function are the mean $\mu$ and the width $\Gamma$. In all cases, $A$ is a normalization factor. In previous studies of $\Upsilon(4 \mathrm{~S})$ decays, the Crystal Ball and ARGUS functions were found to provide a good description of the $m_{\mathrm{ES}}$ mass spectra of signal and continuum background events, respectively. The Breit-Wigner function is well known to describe 
the mass spectra of hadronic resonances such as the $K^{* 0}$.

The rooFit package was used to fit trial PDFs to the $m_{\mathrm{ES}}, \Delta E$, and $m_{K^{* 0}}$ distributions of the signal and background MC samples. The PDF resulting in the smallest $\chi^{2}$ with respect to the distribution was selected. The $B \bar{B}$ background was divided into four categories as explained in Sect. 3.6. Separate PDFs were determined for each category.

\section{Signal PDFs}

The signal MC events remaining after the selection criteria had been applied were used to determine the three signal PDFs.

For the $\Delta E$ distribution, a sum of two Gaussians, one narrow and the other wide, provided the best fit. The mean and standard deviation of the narrow Gaussian are 0.00218 and $0.0182 \mathrm{GeV}$, respectively. Those of the wide Gaussian are 0.002 and $0.091 \mathrm{GeV}$. The fraction of the narrow Gaussian, i.e. the relative weight given to the narrow Gaussian compared to the wide Gaussian by rooFit, is 0.892 . The $\chi^{2} /$ per

degree-of-freedom (d.o.f.) of the fit was 1.06. Fig. 3.24a shows the $\Delta E$ distribution of the signal MC with its fitted PDF.

For the $m_{\mathrm{ES}}$ distribution, a Crystal Ball function with parameters $\alpha=1.87, \mu=$ $5.28 \mathrm{GeV} / c^{2}, n=2.12$ and $\sigma=0.00266 \mathrm{GeV} / c^{2}$ provided the best fit, with a $\chi^{2} /$ d.o.f. of 0.24 . Fig. 3.24b shows the $m_{\mathrm{ES}}$ distribution of the signal $\mathrm{MC}$ with its fitted PDF. For the $m_{K^{* 0}}$ distribution, the best fit was provided by a Breit-Wigner function 
with mean and width 0.896 and $0.0537 \mathrm{GeV} / \mathrm{c}^{2}$. The $\chi^{2} /$ d.o.f. was 1.3 . Fig. $3.24 \mathrm{c}$ shows the $m_{K^{* 0}}$ distribution of the signal MC and its fitted PDF.

\section{Continuum Background PDFs}

After applying the selection criteria, 1,003 events remained in the generic continuum MC samples ( $u \bar{u}, d \bar{d}, s \bar{s}$ and $c \bar{c}$ events combined, see Table 3.2).

For $\Delta E$, first and second order polynomials were chosen as trial PDFs. A first order polynomial with coefficient $-1.02 \mathrm{GeV}^{-1}$ provided the best fit, with a $\chi^{2} /$ d.o.f. of 0.63. Figure 3.25a shows $\Delta E$ for the continuum MC sample, along with its fitted polynomial function.

For $m_{\mathrm{ES}}$, an ARGUS function with ARGUS parameter $\xi=-15.60$ provided the best fit, with a $\chi^{2} /$ d.o.f. of 0.99 . The cut-off $x_{\max }$. was fixed to half the c.m. energy, $5.29 \mathrm{GeV} / c^{2}$, in this fit. Figure $3.25 \mathrm{~b}$ shows the $m_{\mathrm{ES}}$ distribution of the continuum MC sample, with its fitted PDF.

The sum of a first order polynomial plus a Breit-Wigner function was fitted to the $m_{K^{* 0}}$ distribution. The mean and width of the Breit-Wigner were fixed to the values found for the $K^{* 0}$ in the fit of the signal MC events (Sect. 3.7.2). The polynomial parameter and the fraction of the polynomial relative to the Breit-Wigner were floated in the fit. The fitted polynomial coefficient was $0.11\left(\mathrm{GeV} / \mathrm{c}^{2}\right)^{-1}$. The fitted fraction of the polynomial was 0.897 . The overall fit had a $\chi^{2} /$ d.o.f. of 1.05 . The sum of a second order polynomial plus a Breit-Wigner was also tested but resulted in a 

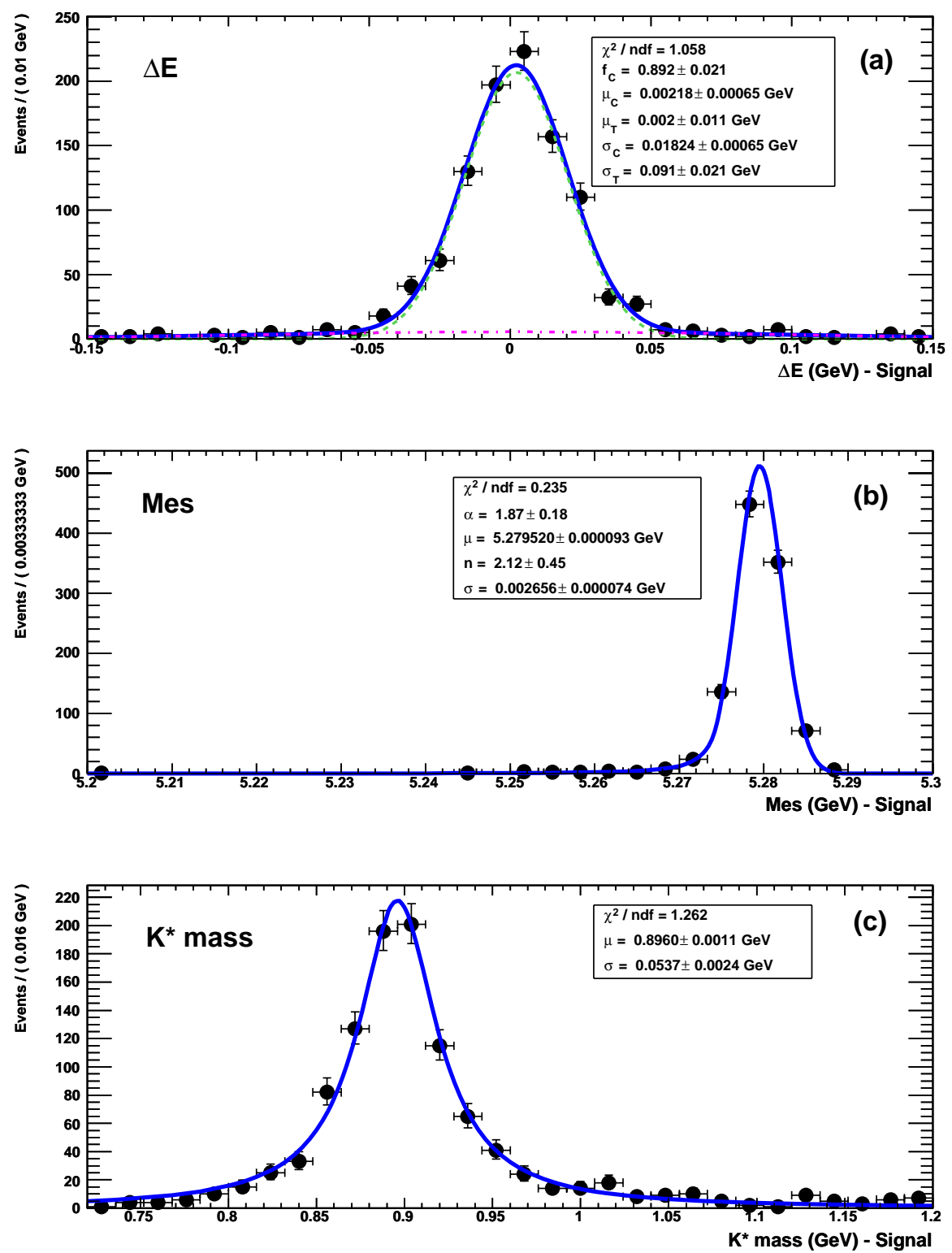

Figure 3.24: Signal PDFs. Plot (a) shows the $\Delta E$ for signal MC (data points) and the fitted two Gaussian PDF (solid line). Plot (b) shows the $m_{\mathrm{ES}}$ for signal MC (data points) and the fitted Crystal Ball PDF (solid line). Plot (c) shows the $K^{* 0}$ invariant mass of signal MC (data points) and its fitted Breit-Wigner PDF (solid line). 
larger $\chi^{2}$. Figure $3.25 \mathrm{c}$ shows the continuum $m_{K^{* 0}}$ distribution along with its fitted PDF.

\section{$B^{0} \bar{B}^{0}$ Background - Category 1 PDFs}

To determine PDFs for the Category $1 B^{0} \bar{B}^{0}$ background, it was not feasible to use the generic $\mathrm{MC}$ because of the small number of events remaining after the cuts were applied (Table 3.3). Instead, a special event sample was constructed using exclusive MC samples of the first three Category 1 channels listed in Table 3.3. The fourth and fifth channels, $B^{0} \rightarrow K_{2}^{* \pm} K^{\mp}$ and $B^{0} \rightarrow K^{\mp} K^{* \pm}$, were not included because their experimental event rates are expected to be negligible as discussed in Sect. 3.6.1. The $B^{0} \rightarrow K_{0}^{* 0}(1430) K_{S}^{0}$ channel is considered separately (Sect. 3.7.2).

Due to limited MC statistics (see Table 3.1) and because of the very low selection efficiency, only $4 B^{0} \rightarrow D^{ \pm} \pi^{\mp}$ events met all of the selection criteria. In order to increase the number of $\mathrm{MC}$ events for this channel, the $K_{S}^{0}$ selection criteria were removed for this channel (Sect. 3.2.6). $182 B^{0} \rightarrow D^{ \pm} \pi^{\mp}$ MC events met these relaxed selection criteria. The $D \pm \pi^{\mp}$ background channel does not contain a $K_{S}^{0}$. Consequently, removing the $K_{S}^{0}$ selection criteria significantly increased the number of events available but should not adversely affect the PDF shapes.

The relative amounts of the three channels was determined by the relative branching fractions times the total selection efficiency. Table 3.5 summarizes the MC samples used to determine the Category 1 PDFs. 

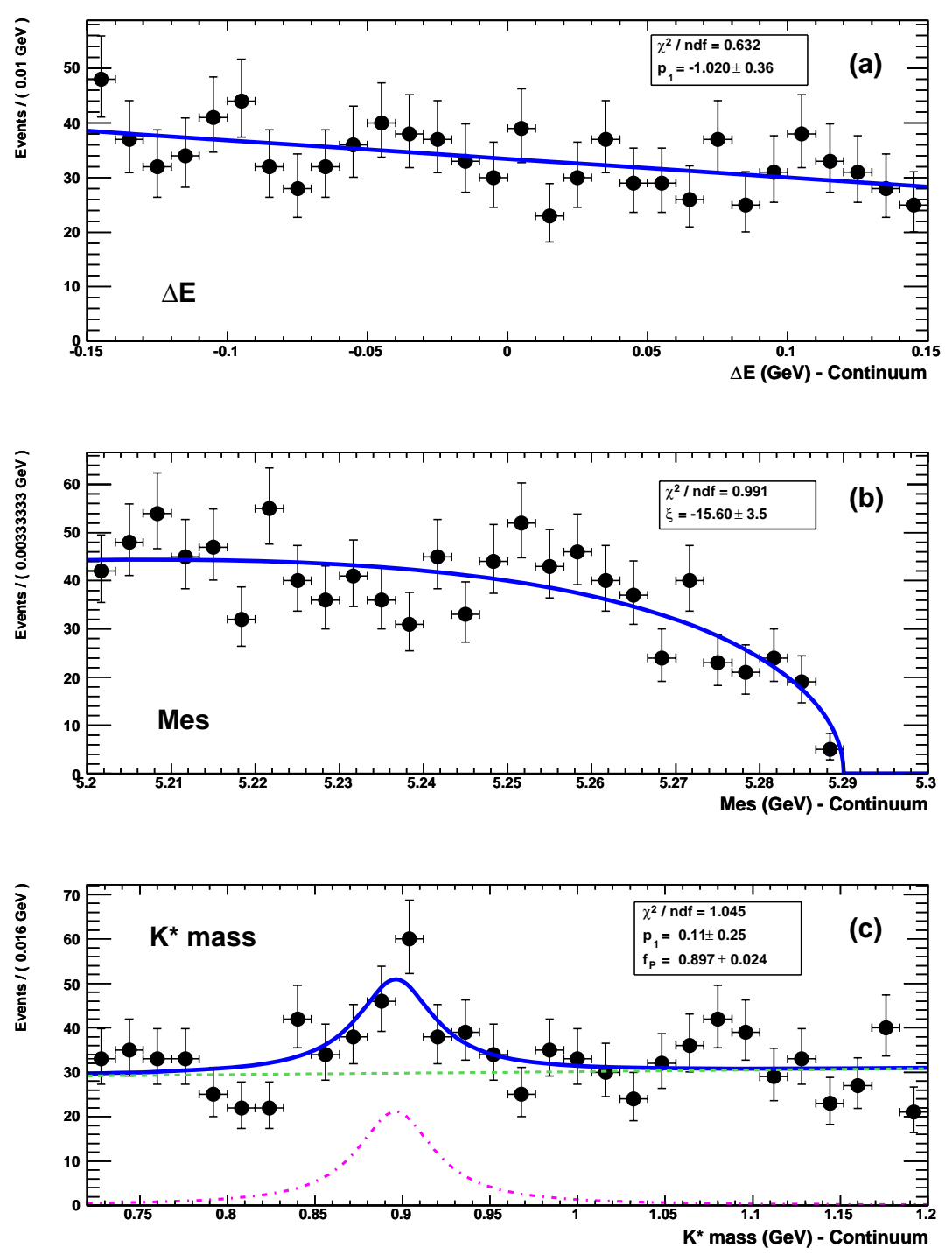

Figure 3.25: Continuum PDFs. Plot (a) shows the $\Delta E$ for continuum MC (data points) and the fitted first order polynomial PDF (solid line). Plot (b) shows the $m_{\mathrm{ES}}$ for continuum MC (data points) and the fitted ARGUS function PDF (solid line). Plot (c) shows the $K^{* 0}$ invariant mass for continuum MC (data points), the fitted first order polynomial plus a Breit-Wigner function PDF (solid line), and the individual components (dashed lines). 
Table 3.5: Composition of the MC sample used for Category $1 B \bar{B}$ related background PDF construction.

\begin{tabular}{|l|c|c|c|c|}
\hline Category 1 Modes & $\begin{array}{c}\text { Branching } \\
\text { Fraction } \\
\left(\times 10^{-6}\right)\end{array}$ & $\begin{array}{c}\text { Selection } \\
\text { Efficiency } \\
\left(\times 10^{-3}\right)\end{array}$ & $\begin{array}{c}\text { Number } \\
\text { in Final } \\
\text { Sample }\end{array}$ & $\begin{array}{c}\text { Fraction } \\
\text { in Final } \\
\text { Sample }\end{array}$ \\
\hline$B^{0} \rightarrow D^{ \pm} K^{\mp}\left(D^{ \pm} \rightarrow \pi^{ \pm} K_{S}^{0}\right)$ & 1.9 & 2.56 & 400 & 0.582 \\
$B^{0} \rightarrow D^{ \pm} \pi^{\mp}\left(D^{ \pm} \rightarrow K^{\mp} \pi^{ \pm} \pi^{ \pm}\right)$ & 258 & 0.00858 & 182 & 0.265 \\
$B^{0} \rightarrow K^{ \pm} \pi^{\mp} K_{S}^{0}$ & 0.5 & 2.56 & 105 & 0.153 \\
\hline
\end{tabular}

As a check on the effect of removing the $K_{S}^{0}$ selection criteria for the $B^{0} \rightarrow D^{ \pm} \pi^{\mp}$ channel, the Category 1 PDFs were also fit using a sample that contained an 80/20 mixture of the $B^{0} \rightarrow D^{ \pm} K^{\mp}$ and $B^{0} \rightarrow K^{ \pm} \pi^{\mp} K_{S}^{0}$ channels plus the $4 B^{0} \rightarrow D^{ \pm} \pi^{\mp}$ events that met all of our selection criteria. The parameter values for the two sets of PDFs generally differed by only a few percent.

For the $\Delta E$ distribution, the sum of a first order polynomial plus a Gaussian was found to provide the best fit. The fitted Gaussian has a mean and width of -0.002 and $0.0138 \mathrm{GeV}$, respectively. The fitted polynomial parameter and fraction of polynomial are $-5.362 \mathrm{GeV}^{-1}$ and 0.856 . The fit yielded a $\chi^{2} /$ d.o.f. of 1.19 . Fig. 3.26a shows the Category $1 \Delta E$ distribution with its fitted PDF.

An ARGUS function plus a Gaussian provided the best fit of the Category $1 m_{\mathrm{ES}}$ distribution. The ARGUS cut-off was fixed at 5.29 $\mathrm{GeV} / \mathrm{c}^{2}$. The mean of the Gaussian was fixed at the $B^{0}$ mass. The fitted ARGUS parameter was -89.72. The fitted Gaussian width was $0.00278 \mathrm{GeV} / \mathrm{c}^{2}$. The fitted fraction of the Gaussian relative to the ARGUS function was 0.283 . The $\chi^{2} /$ d.o.f. for the fit was 0.97 . Fig. 3.26b shows the Category $1 m_{\mathrm{ES}}$ distribution and its fitted PDF. 

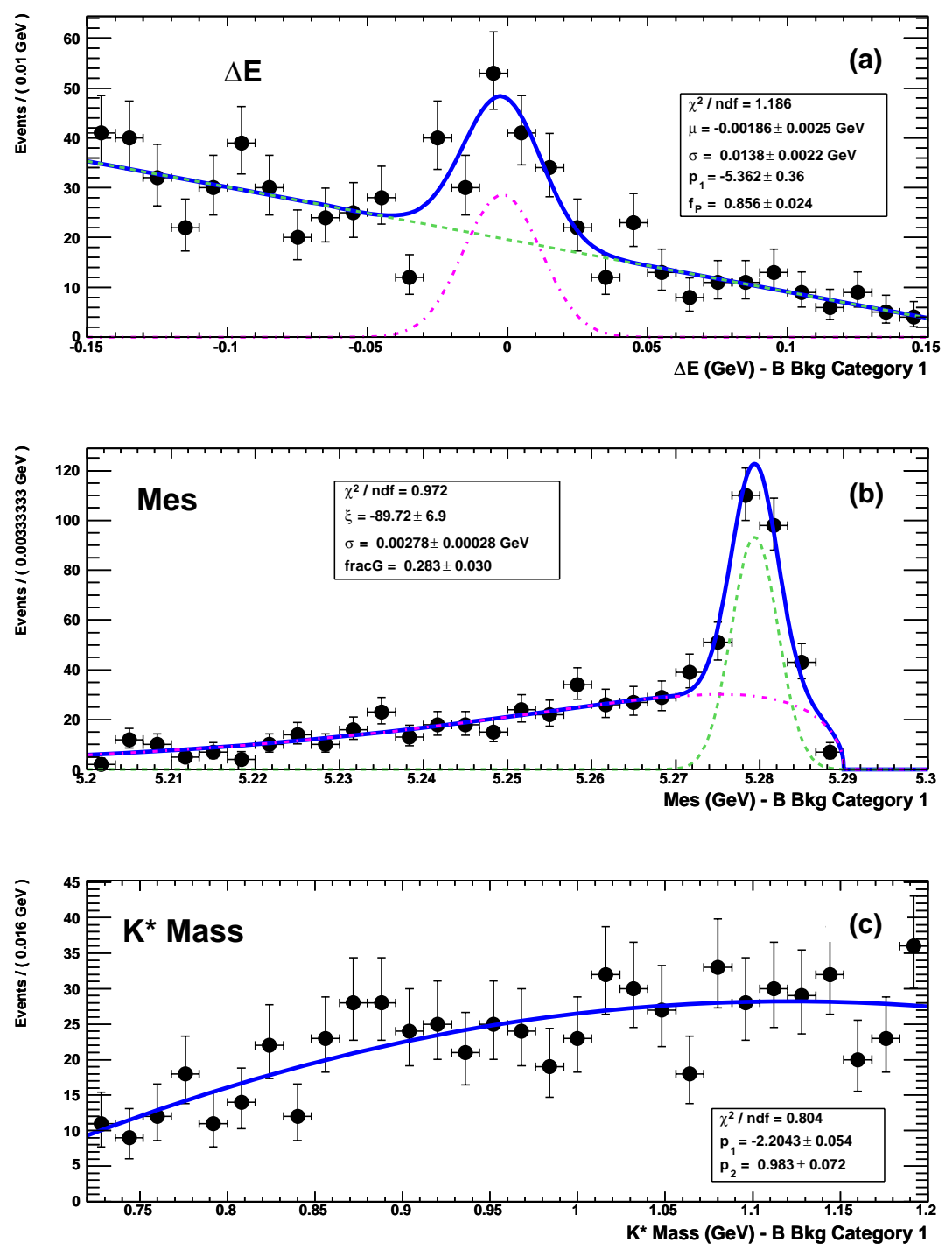

Figure 3.26: Peaking $B^{0} \bar{B}^{0}$ Category 1 background PDFs. Plots (a), (b), and (c) show the $\Delta E, m_{\mathrm{ES}}$, and the $m_{K^{* 0}}$ distributions respectively. The data points are the $B^{0} \bar{B}^{0}$ Category 1 modes MC. The solid line is the fitted PDF. The dashed lines are the individual components of the PDF. 
For the $m_{K^{* 0}}$ distribution, the best fit was provided by a second order polynomial with coefficients $-2.20\left(\mathrm{GeV} / c^{2}\right)^{-1}$ and $0.983\left(\mathrm{GeV} / c^{2}\right)^{-2}$. The $\chi^{2} /$ d.o.f. of the fit was 0.80. Fig. 3.26c shows the $m_{K^{* 0}}$ distribution of the MC sample and its fitted PDF.

\section{$B \bar{B}$ Background $-K_{0}^{* 0}(1430)$ and other S-wave PDFs}

This background category includes the $K_{0}^{* 0}(1430)$ resonance plus other non-resonant S-wave components. Due to lack of experimental information, an appropriate MC sample was not available to reliably evaluate the PDF's for this background category. However, this background mode is expected to behave similarly to signal with respect to $m_{\mathrm{ES}}$ and $\Delta E$. Therefore the signal PDF's for these two parameters were used for this background mode. The S-wave $m_{K^{* 0}}$ distribution has been studied but a consistent line shape has not been determined [38]. The LASS lineshape [37] is considered to be the best available estimate for this lineshape [38]. A MC sample of 10,000 events was generated based on the LASS line shape, and the portion of this sample within our $m_{K^{* 0}}$ Fit Window was used to define the PDF for this parameter.

The best fit to this MC sample was found using a second order polynomial with first and second order coefficients of -3.319 and 4.7 , respectively, yielding a $\chi^{2} /$ d.o.f. of 1.74. Figure 3.27 shows MC distribution (data points) and the fitted PDF for $m_{K^{* 0}}$. 


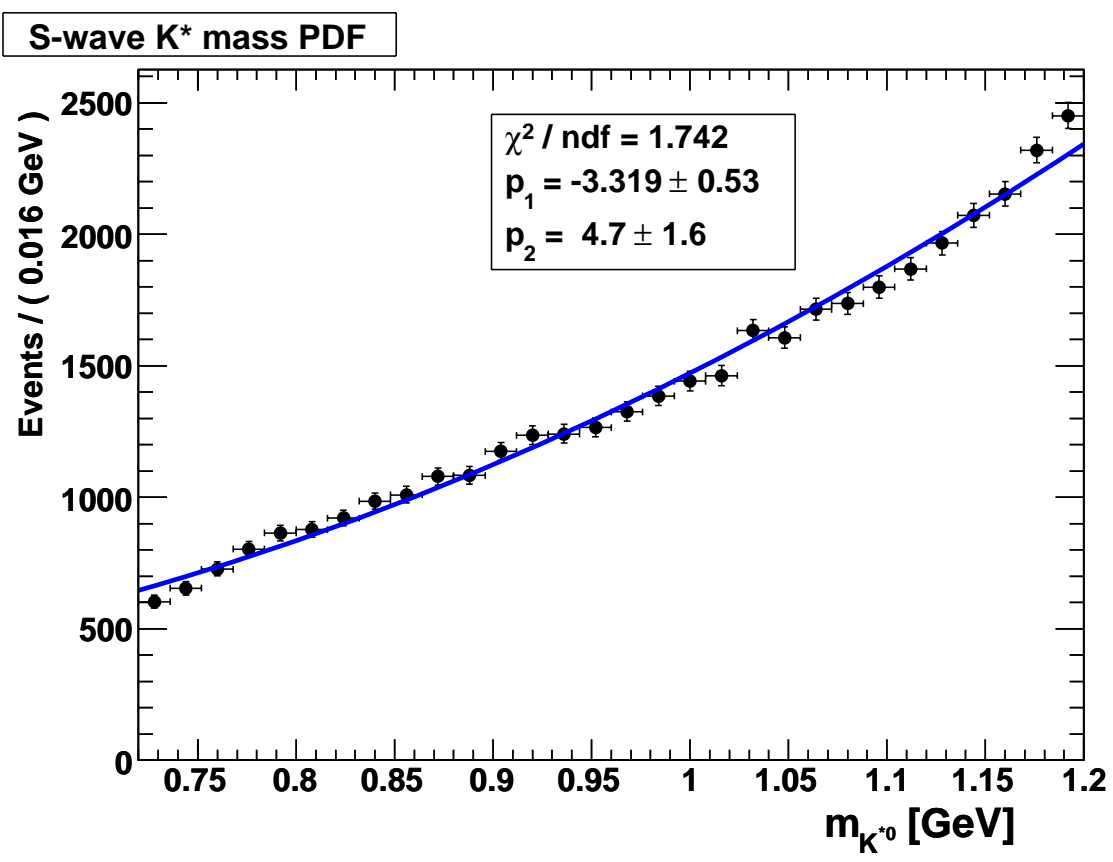

Figure 3.27: $K_{0}^{* 0}(1430)$ and other non-resonant S-wave $m_{K^{* 0}}$ PDF.

\section{$B^{0} \bar{B}^{0}$ Background - Category 2 PDFs}

Exclusive MC samples (Table 3.1) of the two decay channels, $B^{0} \rightarrow \phi K_{S}^{0}$ and $B^{0} \rightarrow$ $f^{0} K_{S}^{0}$, were used to develop the PDFs for this background category. After applying the selection criteria, the relative fraction of each channel was determined by the product of the branching fraction times the selection efficiency. Table 3.5 summarizes the MC samples used to determine the Category 2 PDFs.

For $\Delta E$, the sum of a Gaussian with mean and width -0.0812 and $0.0267 \mathrm{GeV}$, plus a first order polynomial with coefficient $-5.14 \mathrm{GeV}^{-1}$, provided the best fit. The $\chi^{2} /$ d.o.f. was 0.53 . The distribution is shown in Fig. 3.28a along with its fitted PDF.

The sum of an ARGUS function plus a Gaussian provided the best fit for the 

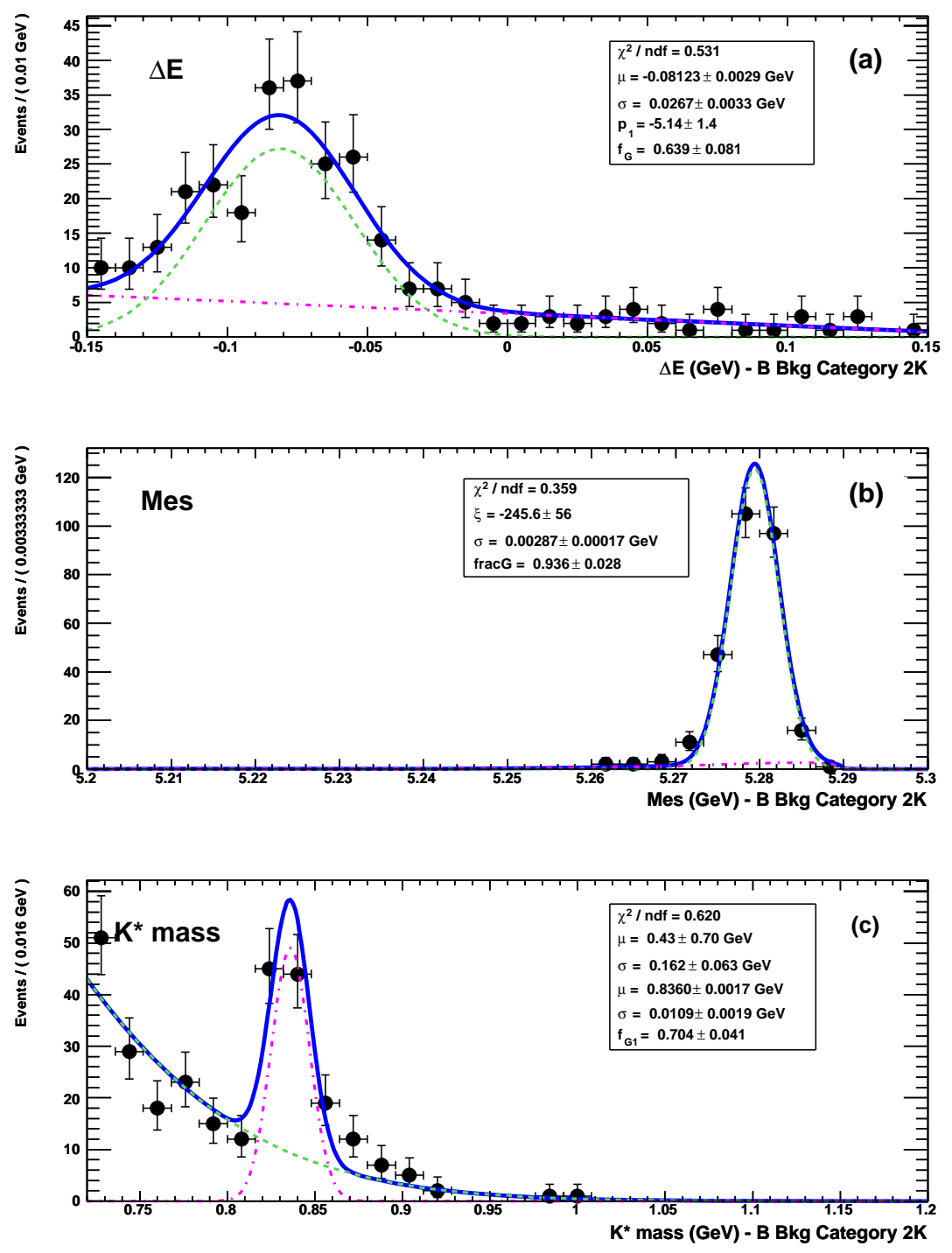

Figure 3.28: Peaking $B^{0} \bar{B}^{0}$ Category 2 background PDFs. Plots (a), (b), and (c) show the $\Delta E, m_{\mathrm{ES}}$, and the $m_{K^{* 0}}$ distributions respectively. The data points are the $B^{0} \bar{B}^{0}$ Category $2 \mathrm{MC}$. The solid line is the fitted PDF. The dashed lines are the individual components of the PDF. 
Table 3.6: Composition of the MC sample used for Category $2 B \bar{B}$ related background PDF construction.

\begin{tabular}{|l|c|c|c|c|}
\hline Category 2 Modes & $\begin{array}{c}\text { Branching } \\
\text { Fraction } \\
\left(\times 10^{-6}\right)\end{array}$ & $\begin{array}{c}\text { Selection } \\
\text { Efficiency } \\
\left(\times 10^{-3}\right)\end{array}$ & $\begin{array}{c}\text { Number } \\
\text { in Final } \\
\text { Sample }\end{array}$ & $\begin{array}{c}\text { Fraction } \\
\text { in Final } \\
\text { Sample }\end{array}$ \\
\hline$B^{0} \rightarrow \phi K_{S}^{0}\left(\phi \rightarrow K^{+} K^{-}\right)$ & 1.42 & 2.14 & 193 & 0.679 \\
$B^{0} \rightarrow f^{0} K_{S}^{0}\left(f^{0} \rightarrow K^{+} K^{-}\right)$ & 0.23 & 6.24 & 91 & 0.321 \\
\hline
\end{tabular}

Category $2 m_{\mathrm{ES}}$ distribution. The ARGUS cut-off was fixed at $5.29 \mathrm{GeV} / \mathrm{c}^{2}$. The mean of the Gaussian was fixed at the $B^{0}$ mass. The fitted ARGUS parameter was -242.5, the Gaussian width $0.00287 \mathrm{GeV} / \mathrm{c}^{2}$, and the fitted fraction of the Gaussian relative to the ARGUS function 0.936. The $\chi^{2} /$ d.o.f. of the fit was 0.36 . Fig. $3.28 \mathrm{~b}$ shows the Category $2 m_{\mathrm{ES}}$ distribution and its fitted PDF.

The best fit of the Category $2 m_{K^{* 0}}$ distribution was provided by the sum of two Gaussians with means 0.43 and $0.836 \mathrm{GeV} / c^{2}$ and widths 0.162 and $0.0109 \mathrm{GeV} / c^{2}$. The fraction of the wider Gaussian relative to the narrower Gaussian is 0.704 . The $\chi^{2} /$ d.o.f. of the fit was 0.62 . Fig. $3.28 \mathrm{c}$ shows the Category $2 m_{K^{* 0}}$ distribution and its fitted PDF. The peak at $0.836 \mathrm{GeV} / c^{2}$ is due to the $\phi K_{S}^{0}$ channel.

\section{$B^{0} \bar{B}^{0}$ Background - Category 3 PDFs}

Exclusive MC samples of the decay channels for the Category $3 B \bar{B}$ background (Table 3.1) were used to construct the PDFs. After applying the selection criteria, the MC samples for each Category 3 channel were combined in proportion to their branching fractions times selection efficiencies. Table 3.7 summarizes the combined event sample used to determine the Category 3 PDFs. 
Table 3.7: Composition of the MC sample used for Category $2-\pi B \bar{B}$ related background PDF construction.

\begin{tabular}{|l|c|c|c|c|}
\hline Category 3 Modes & $\begin{array}{c}\text { Branching } \\
\text { Fraction } \\
\left(\times 10^{-6}\right)\end{array}$ & $\begin{array}{c}\text { Selection } \\
\text { Efficiency } \\
\left(\times 10^{-3}\right)\end{array}$ & $\begin{array}{c}\text { Number } \\
\text { in Final } \\
\text { Sample }\end{array}$ & $\begin{array}{c}\text { Fraction } \\
\text { in Final } \\
\text { Sample }\end{array}$ \\
\hline$B^{0} \rightarrow D^{ \pm} \pi^{\mp}\left(D^{ \pm} \rightarrow \pi^{ \pm} K_{S}^{0}\right)$ & 27 & 0.097 & 217 & 0.296 \\
$B^{0} \rightarrow \rho^{0} K_{S}^{0}\left(\rho^{0} \rightarrow \pi^{ \pm} \pi^{\mp}\right)$ & 2.6 & 1.44 & 310 & 0.423 \\
$B^{0} \rightarrow f^{0} K_{S}^{0}\left(f^{0} \rightarrow \pi^{+} \pi^{-}\right)$ & 1.1 & 1.05 & 96 & 0.131 \\
$B^{0} \rightarrow \pi^{\mp} K_{2}^{* \pm}\left(K_{2}^{* \pm} \rightarrow \pi^{ \pm} K_{S}^{0}\right)$ & $<2.1$ & 0.63 & 110 & 0.150 \\
\hline
\end{tabular}

For $\Delta E$, the sum of a Gaussian with mean and width 0.0536 and $0.0254 \mathrm{GeV}$, plus a first order polynomial with coefficient $0.62 \mathrm{GeV}^{-1}$, provided the best fit. The fraction of polynomial relative to Gaussian is 0.444 . The $\chi^{2} /$ d.o.f. was 0.68 . Fig. $3.29 \mathrm{a}$ shows the Category $3 \Delta E$ distribution and its fitted PDF.

The sum of an ARGUS function plus a Gaussian provided the best fit of the Category $3 m_{\mathrm{ES}}$ distribution. The ARGUS cut-off was fixed at $5.29 \mathrm{GeV} / c^{2}$. The mean of the Gaussian was fixed at the $B^{0}$ mass. The fit yielded an ARGUS parameter of -73.52 , a Gaussian width of $0.00294 \mathrm{GeV} / c^{2}$, and the fraction of the Gaussian relative to the ARGUS function of 0.548 . The $\chi^{2} /$ d.o.f. was 0.40 . Fig. 3.29b shows the Category $3 m_{\mathrm{ES}}$ distribution and its fitted PDF.

The best fit of the Category $3 m_{K^{* 0}}$ distribution was provided by the sum of a first order polynomial with coefficient $-1.622\left(\mathrm{GeV} / c^{2}\right)^{-1}$, plus two Gaussians with means 1.145 and $0.891 \mathrm{GeV} / c^{2}$ and widths 0.043 and $0.0396 \mathrm{GeV} / c^{2}$. The fraction of the first Gaussian is 0.154 while that of the second Gaussian is 0.148 . The $\chi^{2} /$ d.o.f. was 0.80 . The Category $3 m_{K^{* 0}}$ and its fitted PDF are shown in Fig. 3.29c. The peak 

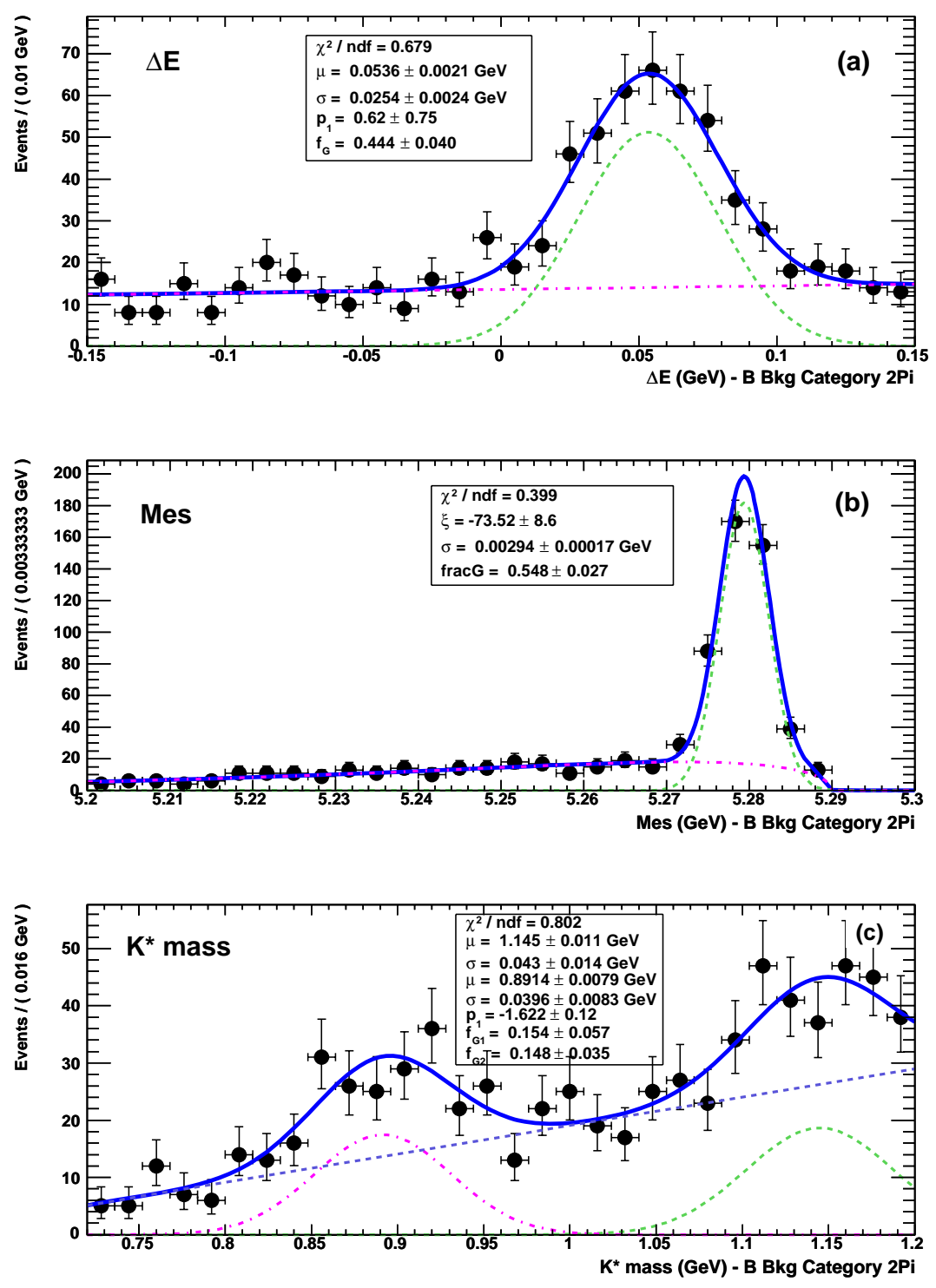

Figure 3.29: Peaking $B^{0} \bar{B}^{0}$ Category 3 background PDFs. Plots (a), (b), and (c) show the $\Delta E, m_{\mathrm{ES}}$, and the $m_{K^{* 0}}$ distributions respectively. The data points are the $B^{0} \bar{B}^{0}$ Category $3 \mathrm{MC}$. The solid line is the fitted PDF. The dashed lines are the individual components of the PDF. 
at $1.145 \mathrm{GeV} / c^{2}$ is from the $B^{0} \rightarrow f^{0} K_{S}^{0}$ channel, while the peak at $0.891 \mathrm{GeV} / c^{2}$ is from the $B^{0} \rightarrow \rho^{0} K_{S}^{0}$ channel.

\section{$B \bar{B}$ Background - Category 4 PDFs}

Category 4 includes $B^{0} \bar{B}^{0}$ and $B^{+} B^{-}$background as explained in Sect. 3.6.5. The combined generic $B^{0} \bar{B}^{0}$ and $B^{+} B^{-}$Category $4 \mathrm{MC}$ samples were used to determine the Category 4 PDFs.

For $\Delta E$, a first order polynomial with coefficient $-3.86 \mathrm{GeV}^{-1}$ provided the best fit. The $\chi^{2} /$ d.o.f. was 0.70. Fig. 3.30a shows the $\Delta E$ distribution and its fitted PDF.

For $m_{\mathrm{ES}}$, a combination of an ARGUS function and a Gaussian provided the best fit. The mean of the Gaussian was fixed at the $B^{0}$ mass. The width of the Gaussian was fixed at the average of the Gaussian widths found for the $m_{\mathrm{ES}}$ distributions in Categories 1, 2, and 3. The ARGUS cutoff parameter was fixed at $5.29 \mathrm{GeV} / \mathrm{c}^{2}$. The fitted ARGUS parameter and fraction of the Gaussian function are -42.75 and 0.097. The $\chi^{2} /$ d.o.f. was 0.74 . Fig. $3.30 \mathrm{~b}$ shows the Category $4 m_{\mathrm{ES}}$ distribution and its fitted PDF.

For $m_{K^{* 0}}$, a second order polynomial with coefficients $-2.476\left(\mathrm{GeV} / c^{2}\right)^{-1}$ and $1.63\left(\mathrm{GeV} / \mathrm{c}^{2}\right)^{-2}$ provided the best fit, yielding a $\chi^{2} /$ d.o.f. of 0.85 . Fig. 3.30c shows the Category 3 distribution of $m_{K^{* 0}}$ with its fitted PDF. 

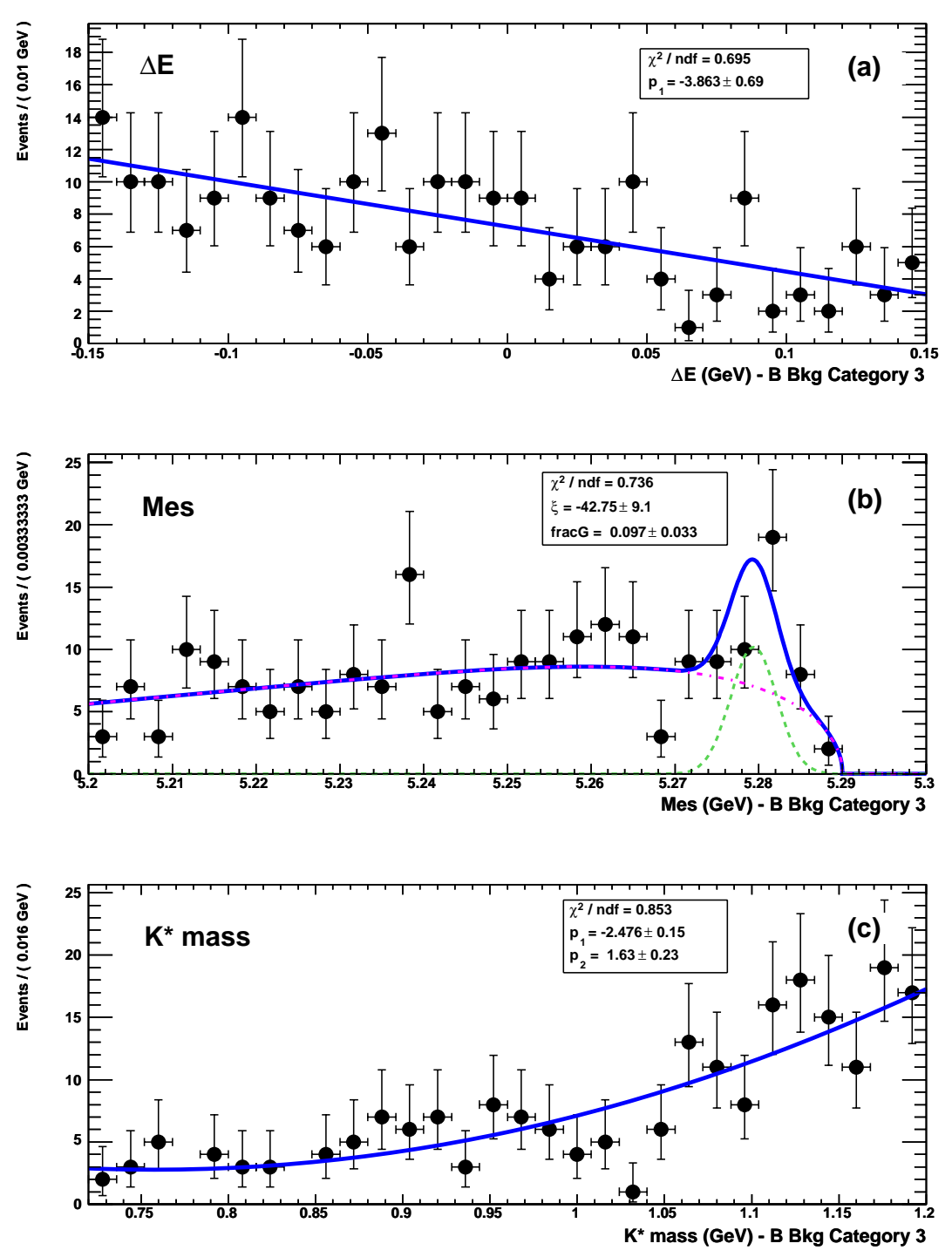

Figure 3.30: $B \bar{B}$ Category 4 PDFs. Plot (a) shows the $\Delta E$ distribution for the Category 4 B background MC (data points) and the fitted PDF (solid line). Plot (b) shows the $m_{\mathrm{ES}}$ distribution for the Category $4 \mathrm{~B}$ background MC (data points), the fitted PDF (solid line), and the ARGUS and Gaussian components (dashed lines). Plot (c) shows the $m_{K^{* 0}}$ distribution for the Category 4 B background MC (data points) and the fitted PDF (solid line). 


\subsubsection{Fitted parameters}

The following parameters were floated in the Maximum Likelihood fit:

- Signal yield,

- Continuum background yield,

- $K_{0}^{* 0}(1430)$ and S-wave background yield

- Category $4 B \bar{B}$ background yield,

- Polynomial parameter in the continuum $\Delta E$,

- ARGUS parameter in the continuum $m_{\mathrm{ES}}$,

- Polynomial parameter in the continuum $K^{* 0}$ mass,

- Polynomial fraction in the continuum $K^{* 0}$ mass.

The yields of background Categories 1,2 and 3 are not fitted in the Maximum

Likelihood procedure because the fits did not converge when $N^{\text {Bcat1 }}, N^{\text {Bcat2 }}$ or $N^{\text {Bcat3 }}$ (see Eq. (3.8)) were allowed to vary. Instead, these three quantities were fixed to their values from the cut-and-count analysis (see Table 3.2), after adjustment to correspond to the integrated luminosity of the data. The values of $N^{\text {Bcat1 }}, N^{\text {Bcat2 }}$ and $N^{\text {Bcat3 }}$ are varied to determine a systematic uncertainty (Sec. 4.2.

Of the various sources of background, only the continuum background contains enough events to allow us to determine the PDF parameters from data, rather than MC. 


\subsection{Fit Validation}

Maximum Likelihood fits can exhibit an intrinsic bias on the order of $1 / \mathrm{N}$, where $\mathrm{N}$ is the number of events. It is therefore important to determine whether the fit procedure yields the correct result on average, i.e. whether it is unbiased. In addition, it is important to determine whether the fit provides a reliable estimate of the statistical uncertainties and to verify that the fit procedure remains stable over a range of signal and background yields.

To perform these checks, a series of studies were performed using both pure and embedded toy MC as well as simulated data samples. Each pure toy study consisted of 1,000 individual toy MC samples. The embedded toy studies used $250 \mathrm{MC}$ samples due to the limited number of MC events available. For the pure toy studies the samples were generated using the PDF distributions described in Sect. 3.7.2. The number of signal events and each type of background was determined using a Poissonian distribution with a given mean. For the embedded toy studies MC events were randomly selected and embedded into the toy samples. The simulated data samples were created by randomly selecting events from the signal and background MC samples.

\subsubsection{Pure Toy Studies}

To determine the sensitivity and stability of the fit procedure to various combinations

of signal and S-wave yields, several pure toy studies were performed where the Pois- 
sonian means for these yields were varied while the other background yields were held constant. The Poissonian means for the background yields were set to the number of generic MC events which survived the selection criteria described in Sects. 3.2 and 3.6 adjusted to a luminosity of $210 \mathrm{fb}^{-1}$ (see Table 3.2). The continuum background yield was taken to be the sum of the $c \bar{c}, u \bar{u}, d \bar{d}$, and $s \bar{s}$ yields (646 events). The $B^{0} \bar{B}^{0}$ and $B^{+} B^{-}$yields from the generic MC totaled 59 events. Based on an analysis of $B$ related backgrounds (Section 3.6, and Table 3.3), one event each was assigned to Categories 1, 2, and 3 and the remaining 56 events were assigned to Category 4. The $K_{0}^{* 0}(1430)$ resonance was not included in the generic MC and was considered separately in the toy studies.

A total of 10 pure toy studies were performed with the Poissonian mean signal and S-wave yields varied between 0 and 15. The RooRarFit package [40] was used to produce and analyze all of the pure toy MC samples. Figures 3.31 through 3.37 and Table 3.8 summarize the results from this group of toy studies.

Figure 3.31a shows the distribution of the fitted number of signal events for the 1,000 pure toy samples. The Poissonian mean number of signal and S-wave events were both set to 3 . The sample mean is 2.88 and the standard deviation 4.31 . The distribution exhibits a slight bias due to an extended tail at low values. The "pull" distribution is defined by

$$
\operatorname{pull}(N)=\frac{N^{f i t}-N^{0}}{\sigma_{N}^{f i t}},
$$

where $N^{0}$ is the Poissonian mean number of generated events in a sample, $N^{f i t}$ is the 
number of events determined by the fit, and $\sigma_{N}^{f i t}$ is the uncertainty of $N^{\text {fit }}$ returned by the fitting program (see Fig. 3.31b). If the mean of a Gaussian function fitted to the pull distribution is zero, the fit is unbiased. If the corresponding standard deviation is unity, the calculated uncertainties are accurate.

Figure 3.31c shows the pull distribution for the number of signal events in the pure toy MC samples. A Gaussian function fitted to this distribution (solid curve) has a mean of -0.123 and a standard deviation of 1.023 , indicating that the fit has a small bias and that the estimated uncertainties are reliable.

Figure 3.32a shows the distribution of the fitted number of signal events for 1,000 pure toy studies. The Poissonian mean number of signal and S-wave events were both set to 15 . The sample mean is 15.09 , in close agreement with the expected number of signal events. The standard deviation is 6.32 . Figure $3.32 \mathrm{~b}$ shows the distribution of the uncertainty on the number of signal events. Figure 3.32c shows the distribution of the corresponding pull. A Gaussian function fitted to this latter distribution (solid curve) has a mean of -0.042 and a width of 1.026 , indicating that the fit is essentially unbiased and that it accurately estimates the uncertainties.

To check for possible biases in the other parameters floated in the fit (Sect. 3.7.3), the corresponding pull distributions were calculated and are shown in Figs. 3.34-3.37. Gaussian functions fitted to all of these pull distributions show means very near zero and standard deviations approximately equal to unity, as reported in the insets of these figures. 

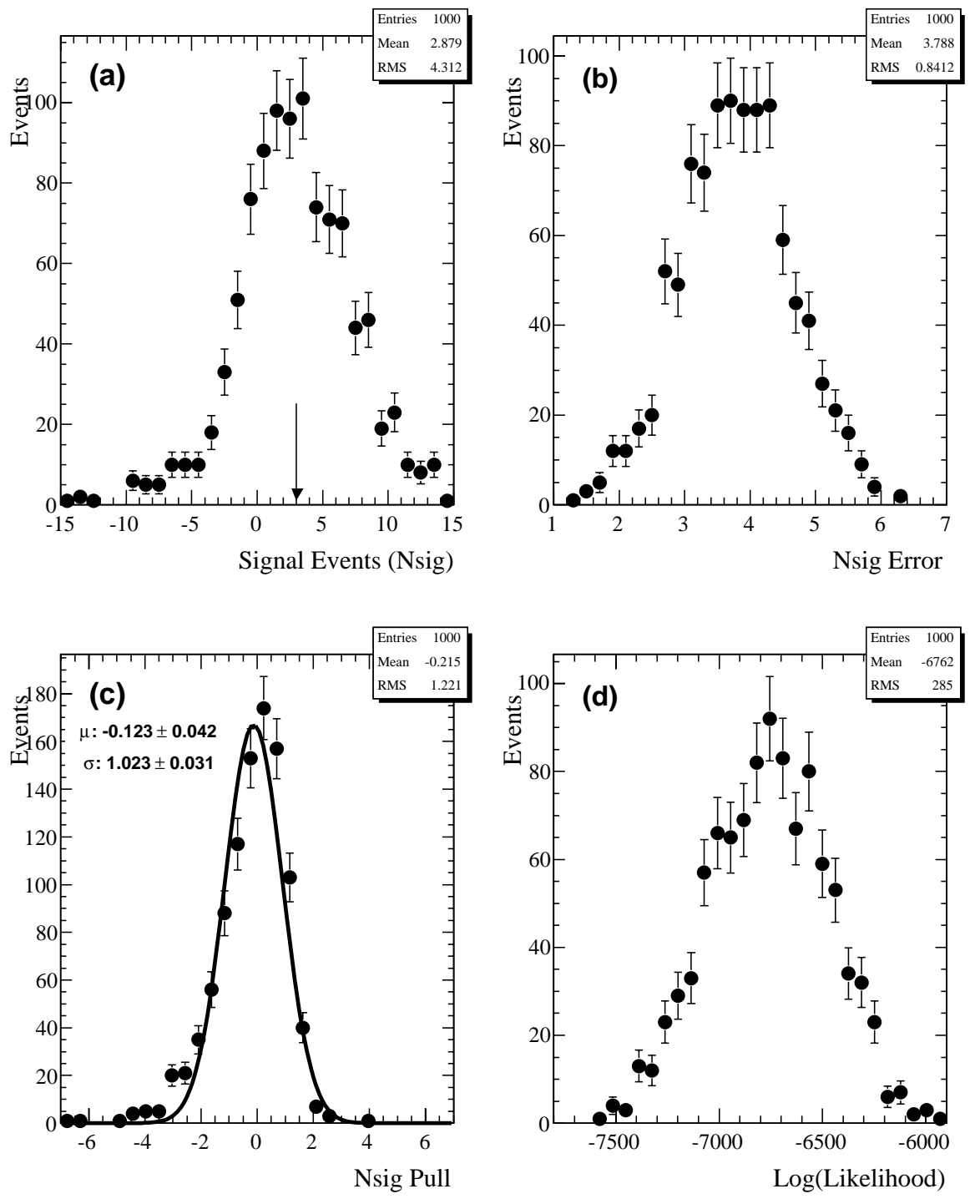

Figure 3.31: Plot (a) shows the distributions of the fitted number of signal events (Nsig). The arrow in plot (a) indicates the value of the Poissonian mean number of signal events. Plot (b) shows the calculated error on the fitted number of signal events (Nsig Error), plot (c) shows the pull as defined in the text, and plot (d) shows the likelihood function for 1,000 pure toy MC samples. 

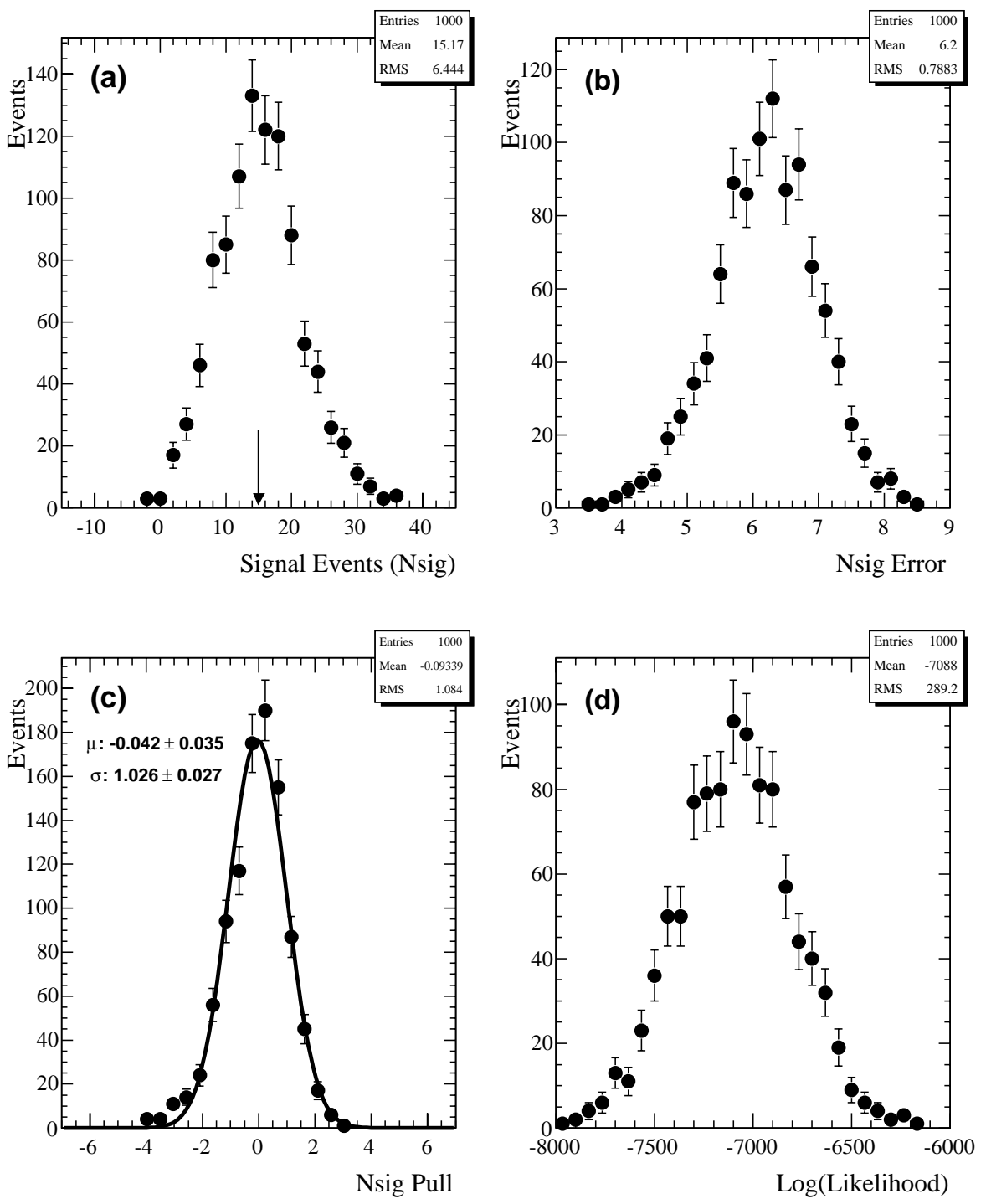

Figure 3.32: Plot (a) shows the distribution of the fitted number of signal events (Nsig); Plot (b), the calculated error on the fitted number of signal events (Nsig Error); Plot (c) the pull distribution; and Figure 3.32 (d) the likelihood function for 1,000 pure toy MC samples. 

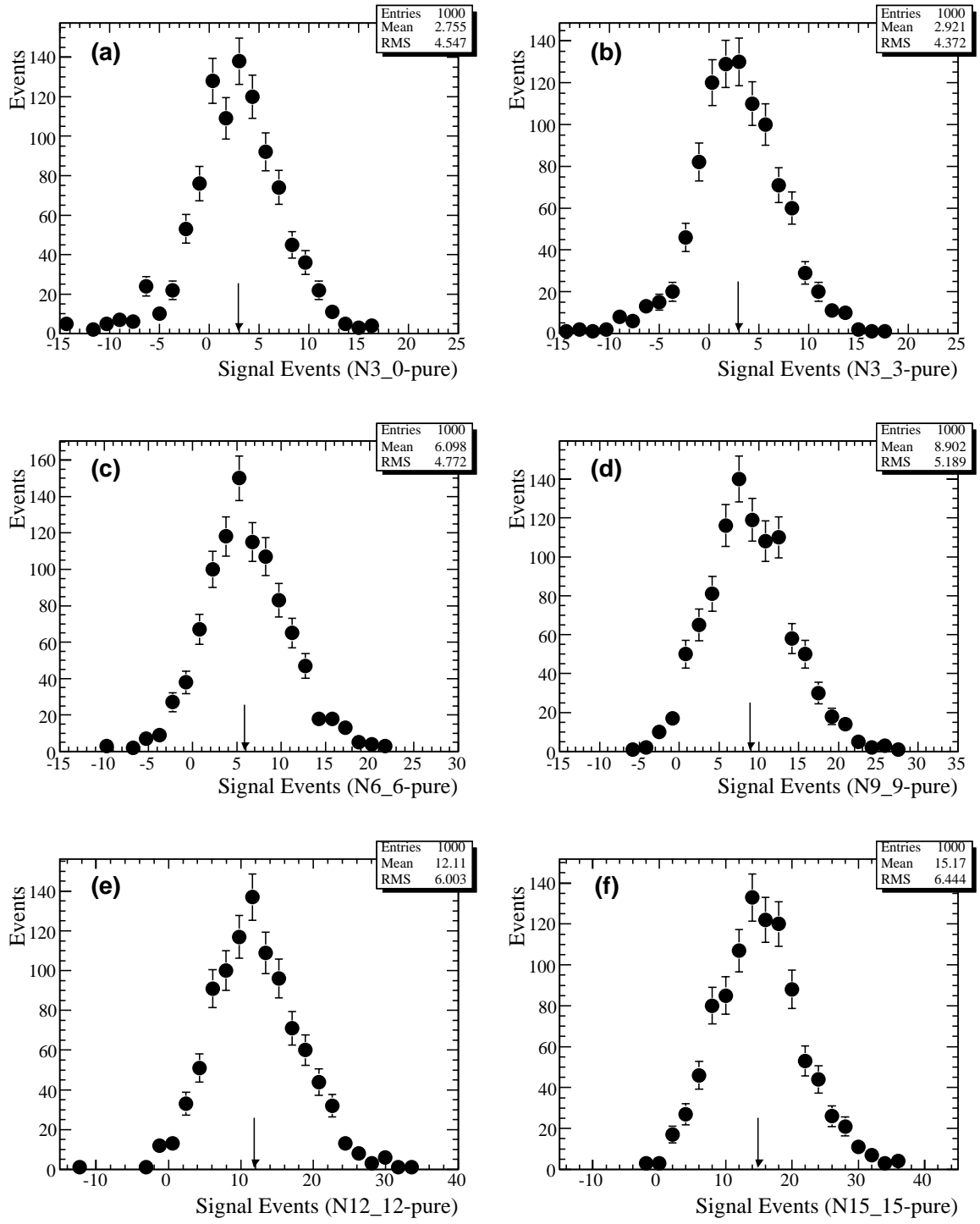

Figure 3.33: The distribution of the fitted number of signal events (Nsig) for several of the pure toy studies. In plot (a) the Poissonian mean number of signal and S-wave events were set to 3 and 0 respectively. In plot (b) the Poissonian mean number of signal and S-wave events were both set to 3 . In plot (c) the Poissonian means were both set to 6 . In plot (d) the Poissonian means were both set to 9 . In plot (e) the Poissonian means were both set to 12 . In plot (f) the Poissonian means were both set to 15 . 
Table 3.8: Results from the pure toy studies with the Poissonian mean signal and S-wave events (input values) varied between 0 and 15

\begin{tabular}{|l|c|c|c|c|}
\hline $\begin{array}{l}\text { Parameter } \\
\text { Input Value) }\end{array}$ & $\begin{array}{c}\text { Toy Output } \\
\text { Mean Yield }\end{array}$ & $\begin{array}{c}\text { Toy Output } \\
\text { Yield Error }\end{array}$ & $\begin{array}{c}\text { Pull Dist. } \\
\text { Mean }\end{array}$ & $\begin{array}{c}\text { Pull Dist. } \\
\text { Width }\end{array}$ \\
\hline Signal (0) & $-0.490 \pm 4.35$ & $3.2 \pm 0.90$ & $-0.089 \pm 0.045$ & $0.994 \pm 0.029$ \\
S-wave (0) & $0.182 \pm 5.40$ & $4.84 \pm 1.05$ & $-0.046 \pm 0.044$ & $1.067 \pm 0.033$ \\
\hline Signal (0) & $-0.443 \pm 4.36$ & $3.31 \pm 0.84$ & $-0.061 \pm 0.047$ & $1.049 \pm 0.040$ \\
S-wave (3) & $3.35 \pm 5.84$ & $5.38 \pm 1.05$ & $-0.023 \pm 0.040$ & $0.965 \pm 0.026$ \\
\hline Signal (0) & $-0.533 \pm 4.22$ & $3.64 \pm 0.85$ & $-0.147 \pm 0.049$ & $0.990 \pm 0.037$ \\
S-wave (12) & $12.26 \pm 7.06$ & $6.63 \pm 0.98$ & $-0.008 \pm 0.036$ & $1.060 \pm 0.032$ \\
\hline Signal (3) & $2.76 \pm 4.55$ & $3.70 \pm 0.83$ & $-0.160 \pm 0.043$ & $1.020 \pm 0.027$ \\
S-wave (0) & $0.107 \pm 5.44$ & $5.00 \pm 0.98$ & $-0.043 \pm 0.043$ & $0.974 \pm 0.027$ \\
\hline Signal (3) & $2.92 \pm 4.37$ & $3.79 \pm 0.84$ & $-0.123 \pm 0.042$ & $1.023 \pm 0.031$ \\
S-wave (3) & $3.15 \pm 5.74$ & $5.33 \pm 1.14$ & $-0.082 \pm 0.037$ & $1.018 \pm 0.026$ \\
\hline Signal (3) & $3.09 \pm 4.55$ & $4.30 \pm 0.83$ & $-0.084 \pm 0.037$ & $1.003 \pm 0.028$ \\
S-wave (12) & $12.02 \pm 6.75$ & $6.79 \pm 0.87$ & $-0.089 \pm 0.036$ & $0.967 \pm 0.023$ \\
\hline Signal (6) & $6.10 \pm 4.77$ & $4.55 \pm 0.81$ & $-0.029 \pm 0.036$ & $0.998 \pm 0.030$ \\
S-wave (6) & $6.04 \pm 6.11$ & $6.07 \pm 0.91$ & $-0.060 \pm 0.034$ & $1.009 \pm 0.027$ \\
\hline Signal (9) & $8.90 \pm 5.19$ & $5.15 \pm 0.77$ & $-0.093 \pm 0.035$ & $0.978 \pm 0.027$ \\
S-wave (9) & $9.27 \pm 6.77$ & $6.57 \pm 0.92$ & $-0.038 \pm 0.039$ & $1.005 \pm 0.027$ \\
\hline Signal (12) & $12.11 \pm 6.00$ & $5.70 \pm 0.79$ & $-0.078 \pm 0.034$ & $1.015 \pm 0.024$ \\
S-wave (12) & $11.88 \pm 6.90$ & $6.98 \pm 0.915$ & $-0.112 \pm 0.033$ & $0.985 \pm 0.025$ \\
\hline Signal (15) & $15.17 \pm 6.44$ & $6.20 \pm 0.79$ & $-0.042 \pm 0.035$ & $1.026 \pm 0.027$ \\
S-wave (15) & $14.76 \pm 7.68$ & $7.45 \pm 0.91$ & $-0.115 \pm 0.035$ & $1.022 \pm 0.023$ \\
\hline
\end{tabular}



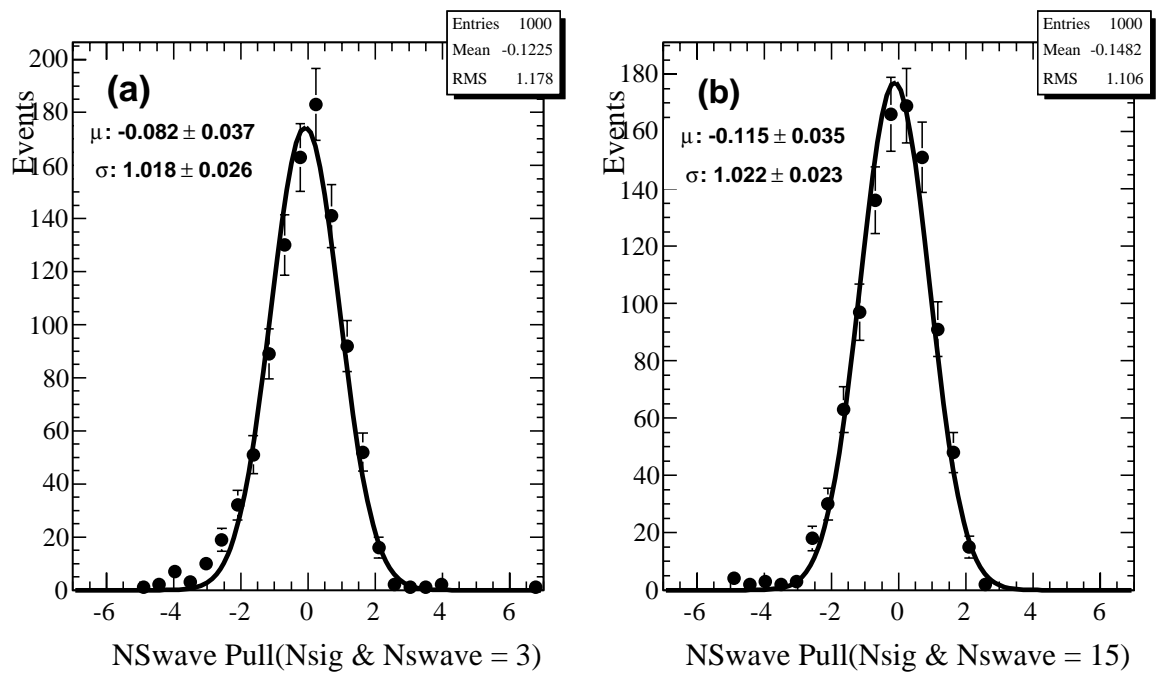

Figure 3.34: Plots (a) and (b) show the pull distributions for S-wave background yield for 1,000 pure toy studies. The Poissonian mean number of signal and S-wave events both set to 3 in plot (a) and 15 in plot (b). 

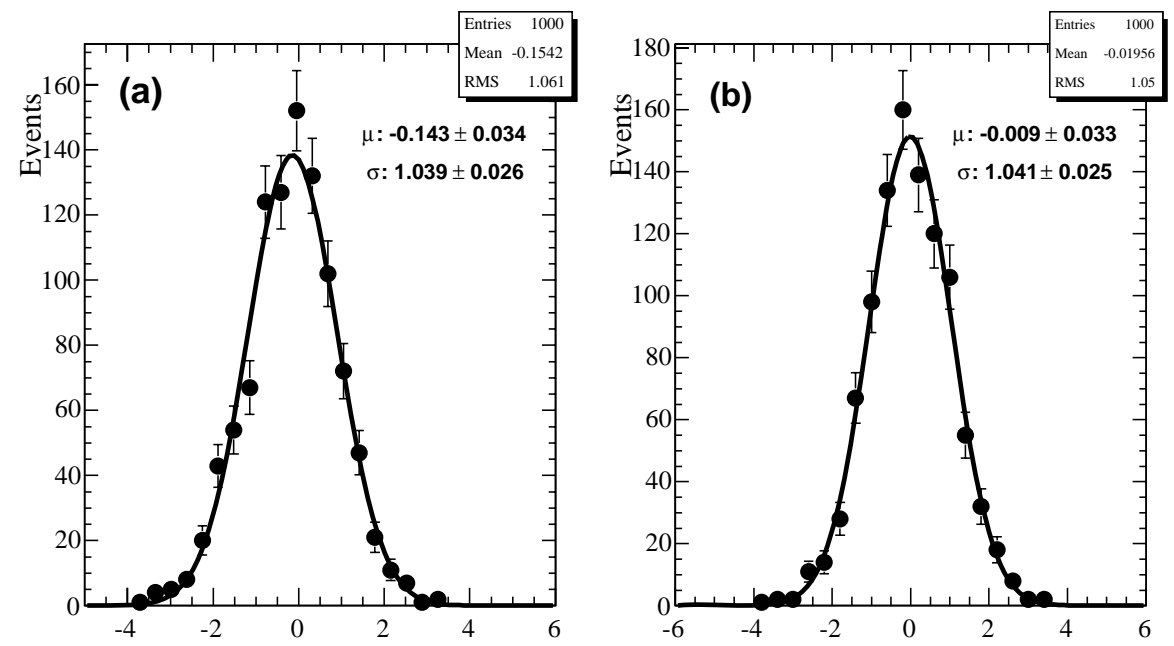

B Bkg Cat 3 (Nsig \& Nswave=3) Pull
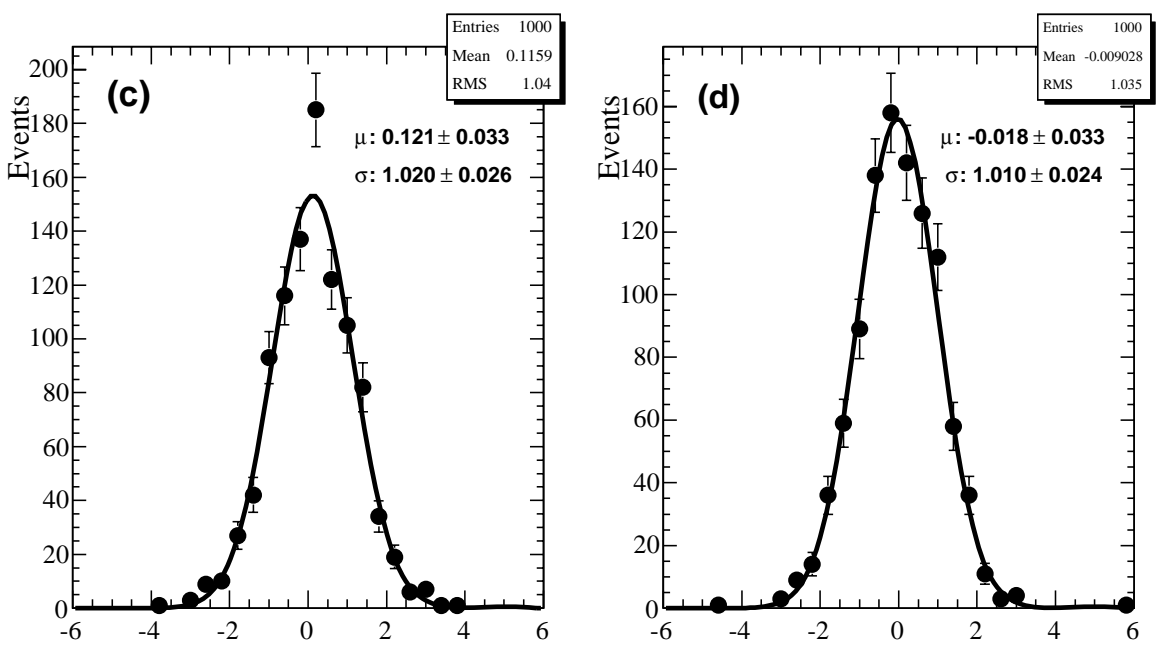

Continuum Bkg (Nsig \& Nswave=3) Pull

Continuum Bkg (Nsig \& Nswave=15) Pull

Figure 3.35: Plots (a) and (b) show the pull distributions for category 3 B related background yield with the Poissonian mean number of signal and S-wave events both set to 3 and 15 respectively. Plots (c) and (d) show the pull distributions for continuum background yield with the same inputs. 

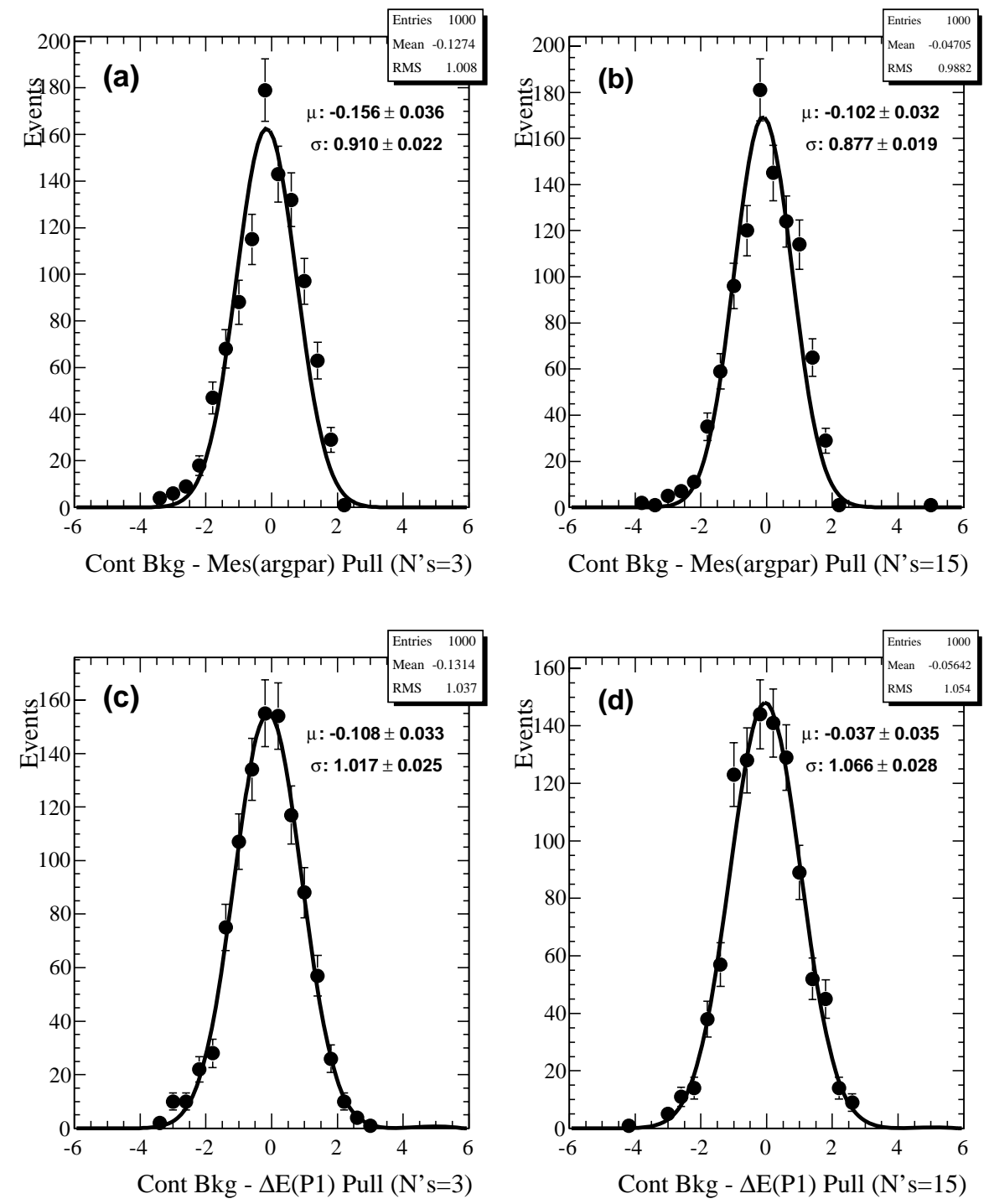

Figure 3.36: The pull distributions for two of the parameters to be floated in the continuum background PDFs based on 1,000 pure toy studies. Plots (a) and (b) show the pull distribution for the ARGUS parameter in the $m_{\mathrm{ES}} \mathrm{PDF}$ with the Poissonian mean number of signal and S-wave events both set to 3 and 15 respectively. Plots (c) and (d) show the pull distribution for the polynomial parameter in the $\triangle E$ PDF with the same inputs. 

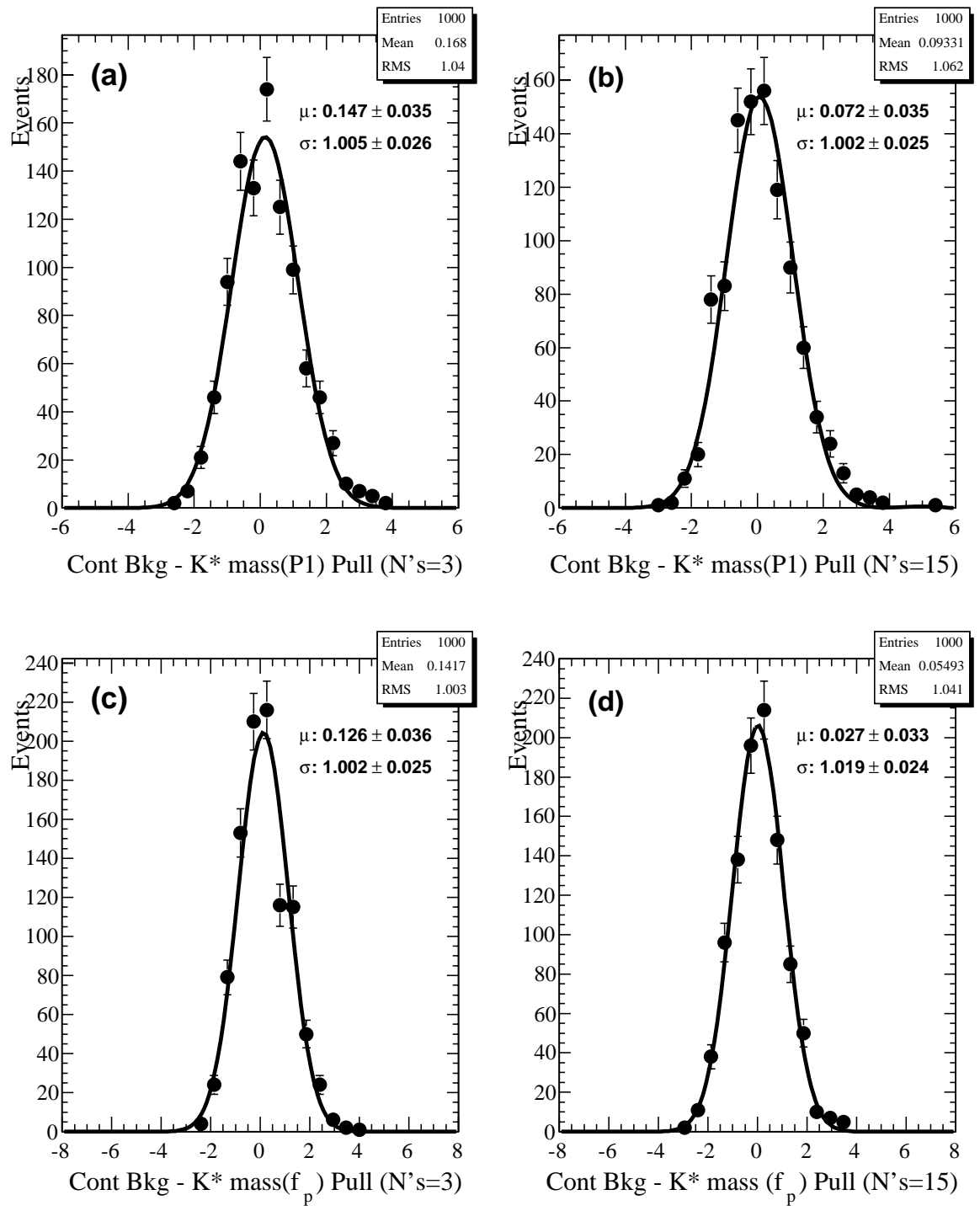

Figure 3.37: The pull distributions for two of the parameters to be floated in the continuum background PDFs based on 1,000 pure toy studies. Plots (a) and (b) show the pull for the polynomial parameter in the $K^{* 0}$ mass PDF with the Poissonian mean number of signal and S-wave events both set to 3 and 15 respectively. Plots (c) and (d) show the pull distribution for the fraction of the polynomial PDF in the total $K^{* 0}$ mass PDF with the same inputs. 
Table 3.9: Poissonian mean inputs used to study the effect of varying background yields.

\begin{tabular}{|l|c|c|c|c|c|c|c|}
\hline $\begin{array}{l}\text { Study } \\
\text { Name }\end{array}$ & $\begin{array}{c}\text { Signal } \\
\text { Mean }\end{array}$ & $\begin{array}{c}\text { S-wave } \\
\text { Mean }\end{array}$ & $\begin{array}{c}\text { Continuum } \\
\text { Mean }\end{array}$ & $\begin{array}{c}\text { Cat 1 } \\
\text { Mean }\end{array}$ & $\begin{array}{c}\text { Cat 2 } \\
\text { Mean }\end{array}$ & $\begin{array}{c}\text { Cat 3 } \\
\text { Mean }\end{array}$ & $\begin{array}{c}\text { Cat 4 } \\
\text { Mean }\end{array}$ \\
\hline Cont-25-prop & 3 & 4.2 & 621 & 1.4 & 1.4 & 1.4 & 78.6 \\
Cont-50-prop & 3 & 5.4 & 596 & 1.8 & 1.8 & 1.8 & 101.2 \\
Cont-25-even & 3 & 8.0 & 621 & 6.0 & 6.0 & 6.0 & 61.0 \\
\hline
\end{tabular}

To determine the stability of the fit procedure to changes in the level of background, a series of pure toy studies was performed where the Poissonian mean signal yield was kept constant and the Poissonian mean for each background yield was varied. The continuum background was reduced by approximately one and two sigma (25 and 50 events) with an equal number of events being distributed among the other background categories. For two of the studies, these events were distributed in proportion to the number of background events in each category. For the third study, 25 events were removed from the continuum and 5 events were added to each of the other background categories. Table 3.9 shows the Poissonian mean yields used for each of these pure toy studies.

The toy study mean yield, yield errors, pull mean and pull width for each of the floated parameters are shown in Table 3.10. The fit procedure remained stable for a two sigma variation in the continuum background yield and the fitted signal yield remained relatively constant. 
Table 3.10: Results from the pure toy studies with varying Background yields.

\begin{tabular}{|c|c|c|c|c|}
\hline $\begin{array}{l}\text { Parameter } \\
\text { (Input Value) }\end{array}$ & $\begin{array}{l}\text { Toy Output } \\
\text { Mean Yield }\end{array}$ & $\begin{array}{l}\text { Toy Output } \\
\text { Yield Error }\end{array}$ & $\begin{array}{c}\text { Pull Dist. } \\
\text { Mean }\end{array}$ & $\begin{array}{c}\text { Pull Dist. } \\
\text { Width }\end{array}$ \\
\hline \multicolumn{5}{|l|}{ Cont-25-prop } \\
\hline Signal (3) & $3.17 \pm 4.31$ & $3.91 \pm 0.83$ & $-0.077 \pm 0.040$ & $1.019 \pm 0.028$ \\
\hline S-wave $(4.2)$ & $4.30 \pm 5.80$ & $5.68 \pm 0.92$ & $-0.033 \pm 0.038$ & $0.946 \pm 0.029$ \\
\hline Continuum (621) & $627.2 \pm 67.9$ & $64.4 \pm 6.02$ & $0.102 \pm 0.033$ & $1.036 \pm 0.025$ \\
\hline B Bkg Cat 4 (78.6) & $72.6 \pm 64.7$ & $61.3 \pm 6.4$ & $-0.125 \pm 0.034$ & $1.059 \pm 0.026$ \\
\hline \multicolumn{5}{|l|}{ Cont-50-prop } \\
\hline Signal (3) & $2.66 \pm 4.48$ & $3.94 \pm 0.84$ & $-0.150 \pm 0.043$ & $1.037 \pm 0.032$ \\
\hline S-wave $(5.4)$ & $5.98 \pm 6.32$ & $6.09 \pm 0.93$ & $-0.004 \pm 0.035$ & $1.023 \pm 0.026$ \\
\hline Continuum (596) & $600.0 \pm 66.8$ & $65.9 \pm 6.46$ & $0.049 \pm 0.033$ & $1.033 \pm 0.027$ \\
\hline B Bkg Cat 4 (101.2) & $95.7 \pm 66.0$ & $63.2 \pm 6.5$ & $-0.085 \pm 0.034$ & $1.058 \pm 0.026$ \\
\hline \multicolumn{5}{|l|}{ Cont-25-even } \\
\hline Signal (3) & $3.04 \pm 4.58$ & $4.15 \pm 0.85$ & $-0.063 \pm 0.035$ & $1.026 \pm 0.029$ \\
\hline S-wave (8.0) & $8.10 \pm 6.78$ & $6.37 \pm 0.94$ & $-0.050 \pm 0.036$ & $1.029 \pm 0.027$ \\
\hline Continuum (621) & $624.9 \pm 63.2$ & $64.4 \pm 6.08$ & $0.059 \pm 0.031$ & $0.975 \pm 0.027$ \\
\hline B Bkg Cat 4 (61) & $58.7 \pm 60.1$ & $61.3 \pm 6.6$ & $-0.046 \pm 0.032$ & $0.988 \pm 0.027$ \\
\hline
\end{tabular}




\subsubsection{Embedded Toy Studies}

To determine the possible bias introduced into our fit procedure because of residual correlations between our input variables $\left(m_{\mathrm{ES}}, \Delta E, m_{K^{* 0}}\right)$, embedded Toy studies were performed. MC events were embedded for signal and all five categories of $\mathrm{B}$ related background. Following the same procedure used for the pure toy studies, the Poissonian mean number of signal and S-wave MC events embedded was varied between 0 and 15. The Poissonian mean number of embedded $\mathrm{MC}$ events for $\mathrm{B}$ background Categories 1, 2, 3, and 4 were set to 1, 1, 1, and 56 respectively. For simplicity, the total number of toy samples was reduced to 250 for this group of studies. Table 3.11 and Figure 3.38 summarize the signal yield results from this group of studies.

Only 217 B background Category 4 MC events were available and each study required 56 events. Consequently, this category was significantly over-sampled in creating 250 Toy samples. The effect of this oversampling is included as part of the systematic uncertainties (Sec. 4.2).

\subsubsection{Event Shape Selection Criteria Evaluation}

The selection criteria used in the analysis were optimized without consideration of the ML fit. In particular, the event shape criteria (the Fisher Discriminant and the $\left.\left|\operatorname{Cos} \theta_{\text {thrust }}\right|\right)$ dramatically reduce the size of the sample used in the fit. A larger sample might lead to a more restrictive upper limit on the branching fraction. To 
Table 3.11: Results from the embedded toy studies with the embedded number of signal and S-wave MC events varied between 0 and 15, and the Poissonian mean number of embedded B background Categories 1, 2, 3, and 4 fixed at 1, 1, 1, and 56 respectively. Each study consisted of 250 toy samples. The embedded B background Category 4 events were drawn from a total sample of only $217 \mathrm{MC}$ events.

\begin{tabular}{|l|c|c|c|c|}
\hline $\begin{array}{l}\text { Parameter } \\
\text { (Input Value) }\end{array}$ & $\begin{array}{c}\text { Toy Output } \\
\text { Mean Yield }\end{array}$ & $\begin{array}{c}\text { Toy Output } \\
\text { Yield Error }\end{array}$ & $\begin{array}{c}\text { Pull Dist. } \\
\text { Mean }\end{array}$ & $\begin{array}{c}\text { Pull Dist. } \\
\text { Width }\end{array}$ \\
\hline $\begin{array}{l}\text { Signal (3) } \\
\text { S-wave (0) }\end{array}$ & $3.40 \pm 4.24$ & $3.76 \pm 0.83$ & $0.192 \pm 0.074$ & $1.051 \pm 0.064$ \\
\hline $\begin{array}{l}\text { Signal (3) } \\
\text { S-wave (3) }\end{array}$ & $3.54 \pm 4.02$ & $3.67 \pm 0.83$ & $0.008 \pm 0.073$ & $1.025 \pm 0.051$ \\
\hline $\begin{array}{l}\text { Signal (6) } \\
\text { S-wave (6) }\end{array}$ & $6.84 \pm 4.43$ & $4.25 \pm 0.80$ & $0.212 \pm 0.070$ & $1.012 \pm 0.065$ \\
\hline $\begin{array}{l}\text { Signal (9) } \\
\text { S-wave (9) }\end{array}$ & $9.15 \pm 5.06$ & $4.59 \pm 0.81$ & $-0.078 \pm 0.078$ & $1.057 \pm 0.060$ \\
\hline $\begin{array}{l}\text { Signal (12) } \\
\text { S-wave (12) }\end{array}$ & $12.71 \pm 5.43$ & $5.14 \pm 0.76$ & $0.052 \pm 0.078$ & $1.046 \pm 0.060$ \\
\hline $\begin{array}{l}\text { Signal (15) } \\
\text { S-wave (15) }\end{array}$ & $16.25 \pm 6.09$ & $5.59 \pm 0.80$ & $0.137 \pm 0.070$ & $1.013 \pm 0.053$ \\
\hline
\end{tabular}



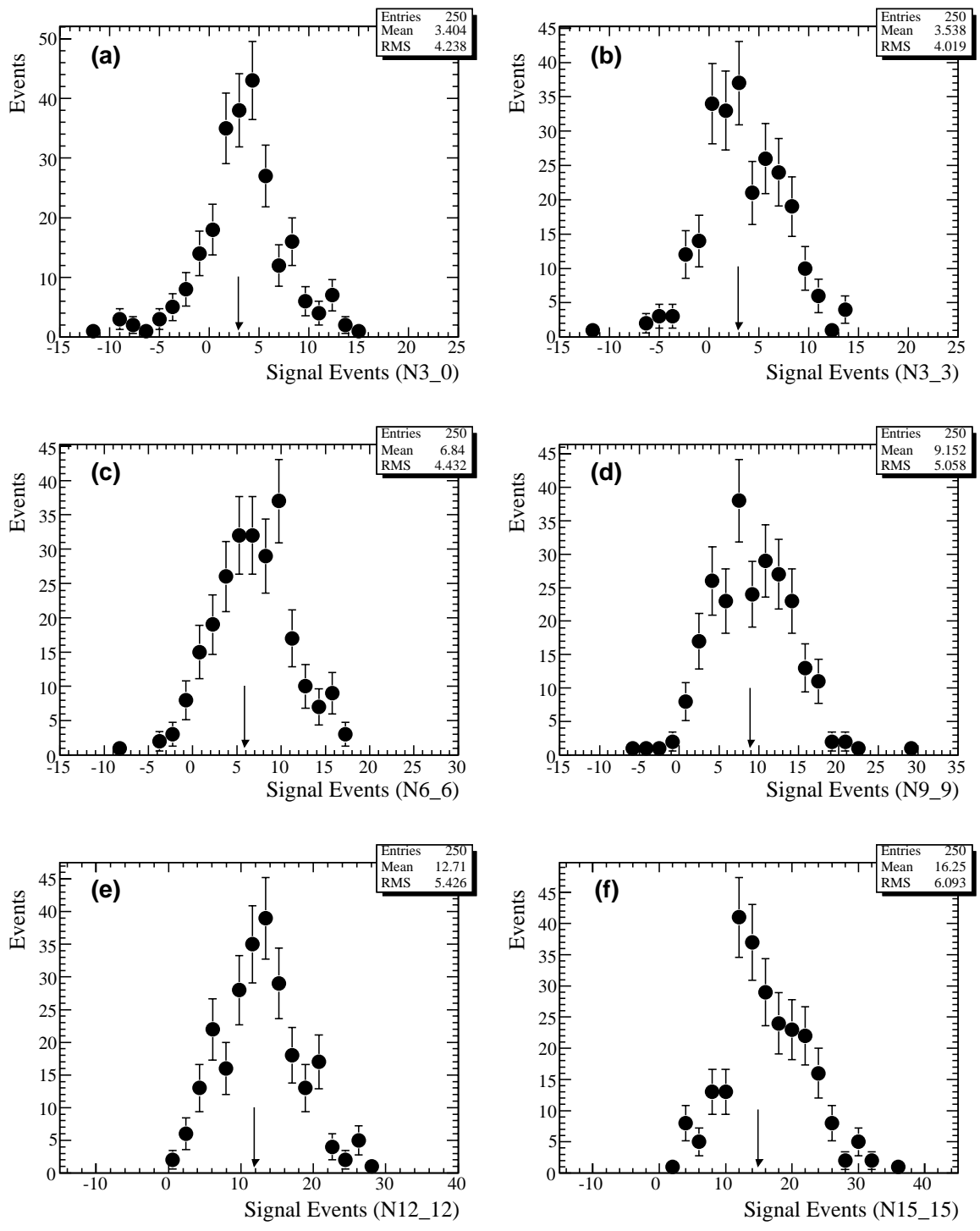

Figure 3.38: The distribution of the fitted number of signal events (Nsig). In plot (a) the Poissonian mean number of embedded signal and S-wave events were set to 3 and 0 respectively. In plot (b) the Poissonian mean number of embedded signal and S-wave events were both set to 3. In plot (c) the Poissonian means were both set to 6. In plot $(\mathrm{d})$ the Poissonian means were both set to 9 . In plot (e) the Poissonian means were both set to 12 . In plot (f) the Poissonian means were both set to 15 . 
evaluate this possibility, new selection criteria for the event shape parameters were developed and evaluated using a series of toy studies.

The maximum allowed $\left|\operatorname{Cos} \theta_{\text {thrust }}\right|$ was increased from $<0.55$ to $<0.90$ and the minimum Fisher Discriminant was reduced from $>0.15$ in steps of 0.05 until the number of signal events passing all the cuts increased by $60 \%$. To achieve a $60 \%$ increase in signal events required a reduction in the minimum Fisher Discriminant value to -0.15. 10,000 signal MC events were used in this study. Of these, 1,060 passed the original criteria (10.6\% efficiency) and 1,706 passed the revised criteria (17.6\% efficiency).

The revised criteria were then used to create new MC samples for all categories of signal and background except $B^{0}$ Category 4 . The process for separating this category from the total generic $B^{0} \mathrm{MC}$ was considered to be too time consuming for the level of precision required for this study. The $B^{0}$ Category 4 background MC from the original selection criteria was combined with the $B^{ \pm} \mathrm{MC}$ after the revised criteria had been applied to form the new Category 4 sample. Table 3.12 shows the number of events passing the revised criteria for each type of generic MC. The new

Table 3.12: The number of signal and generic MC events passing the revised and original Selection Criteria.

\begin{tabular}{|c|c|c|c|c|c|}
\hline Sample & Signal & $c \bar{c}$ & uds & $B^{0} \bar{B}^{0}$ & $B^{ \pm}$ \\
\hline Original Criteria & 1,060 & 411 & 592 & 153 & 120 \\
\hline Revised Criteria & 1,706 & 1,534 & 1,896 & 284 & 218 \\
\hline Original Criteria Adj $210 \mathrm{fb}^{-1}$ & 5 & 269 & 384 & 33 & 26 \\
\hline Revised Criteria Adj $210 \mathrm{fb}^{-1}$ & 9 & 1,004 & 1,229 & 61 & 47 \\
\hline
\end{tabular}


MC samples were then used to define new PDFs for all categories and a series of embedded toy studies was performed to evaluate the effect of the revised selection criteria on the branching fraction upper limit.

200 toy studies were generated for both the revised and original selection criteria. The Poissonian mean number of signal events was set to 9 for the samples using the revised selection criteria and 5 for the samples using the original criteria. An ML fit was performed on each sample to obtain the negative log likelihood (NLL) function for that sample. The NLL function was then inverted and normalized to unit area to obtain the likelihood as a function of the number of signal events. This function was then integrated to obtain a $90 \%$ upper limit on the number of signal events for that sample. The distribution of upper limits for the 200 studies using the revised and original selection criteria are shown in Figures 3.39 and 3.40 respectively.

The mean signal event upper limit for the 200 embedded toy samples was $20.05 \pm$ 6.9 for the revised selection criteria and $12.31 \pm 4.6$ for the original criteria. These upper limits were then used to compute mean upper limits on the branching fraction for the revised and original selection criteria. The signal efficiency, after efficiency corrections and accounting for the final state branching fractions, is $3.80 \%$ for the revised selection criteria and $2.36 \%$ for the original criteria.

The two branching fraction limits are shown below:

- $90 \%$ UL on $\mathcal{B}$ revised criteria $=(2.28 \pm 0.79) \times 10^{-6}$

- $90 \% \mathrm{UL}$ on $\mathcal{B}$ original criteria $=(2.25 \pm 0.84) \times 10^{-6}$

The revised selection criteria result in an upper limit on the branching fraction 


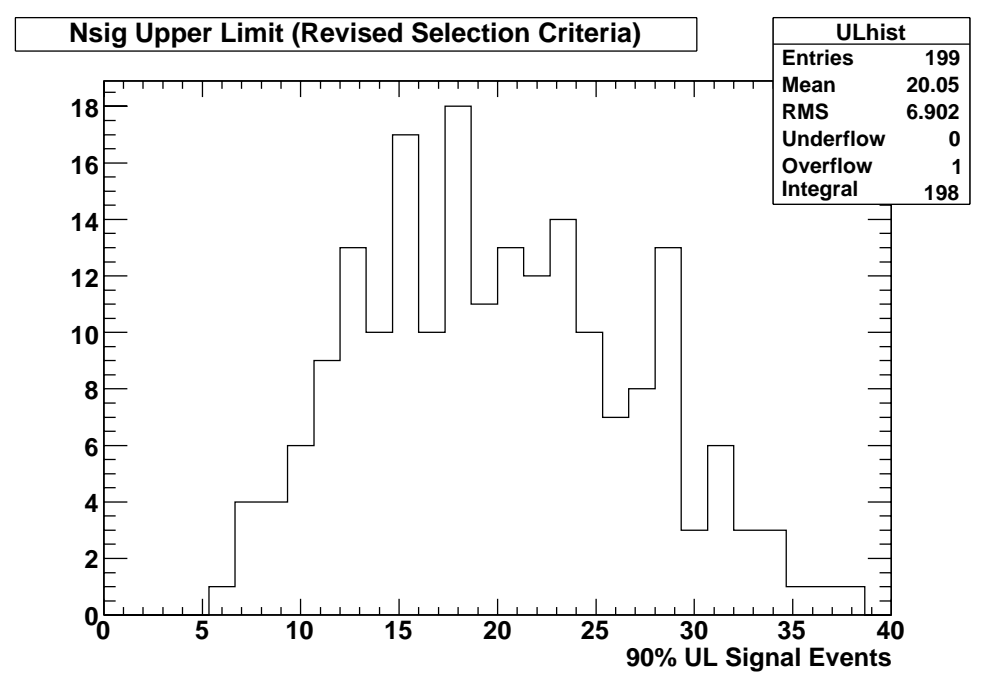

Figure 3.39: Distribution of the upper limit on the number of signal events from 200 embedded toy studies using the revised selection criteria.

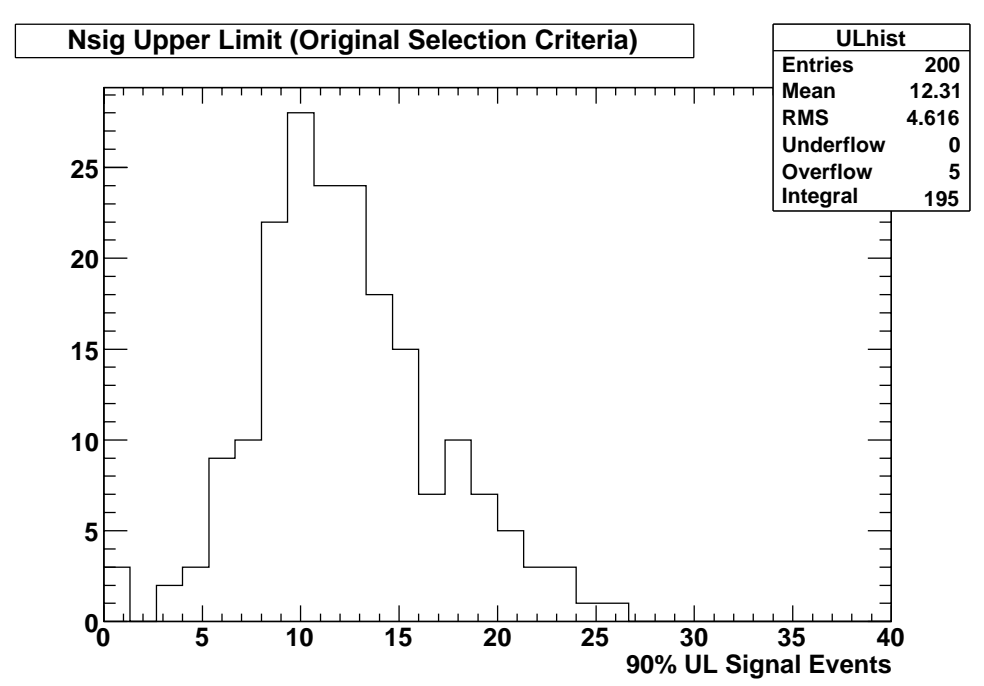

Figure 3.40: Distribution of the upper limit on the number of signal events from 200 embedded toy studies using the original selection criteria. 
that is essentially identical to the limit using the original criteria and no revisions to the selection criteria are necessary.

\subsubsection{Simulated Data Set Studies and Blind Fit}

To further test the validity of the PDFs and fit procedure, test samples were prepared by randomly selecting events from each MC sample. The number of events selected from each MC sample was based on the expected numbers from Table 3.2, adjusted for luminosity. Two samples were prepared, one sample with the number of signal and S-wave events both set to 3 and and the other with them both set to 15. Each of these samples had $1 B^{0} \bar{B}^{0}$ Category 1 event, $1 B^{0} \bar{B}^{0}$ Category 2 event, $1 B^{0} \bar{B}^{0}$ Category 3 event, $56 B^{0} \bar{B}^{0}$ Category 4 events, and 646 continuum events, randomly selected from their respective MC samples. Figure 3.41 shows projection plots for $\Delta E, m_{\mathrm{ES}}$, and the $K^{* 0}$ mass for these two samples. To enhance the signal in these plots, it was required that the log likelihood ratio (LLR), defined as the ratio between the $\log$ likelihood for the signal to the total $\log$ likelihood, $\mathrm{L}(\mathrm{S}) /[\mathrm{L}(\mathrm{S})+\mathrm{L}(\mathrm{B})]$, be larger than 0.60 .

Figure 3.42 shows the distribution of the LLR for each component PDF (signal, continuum, and B related Background - Figures $3.42 \mathrm{a}$ and c). If the fit procedure works correctly, the signal events should be concentrated near an LLR of 1 while the background events are distributed between 0 and 1 . Figures $3.42 \mathrm{~b}$ and d show the total product PDFs (histogram) and the test MC samples (data points). If the fit 

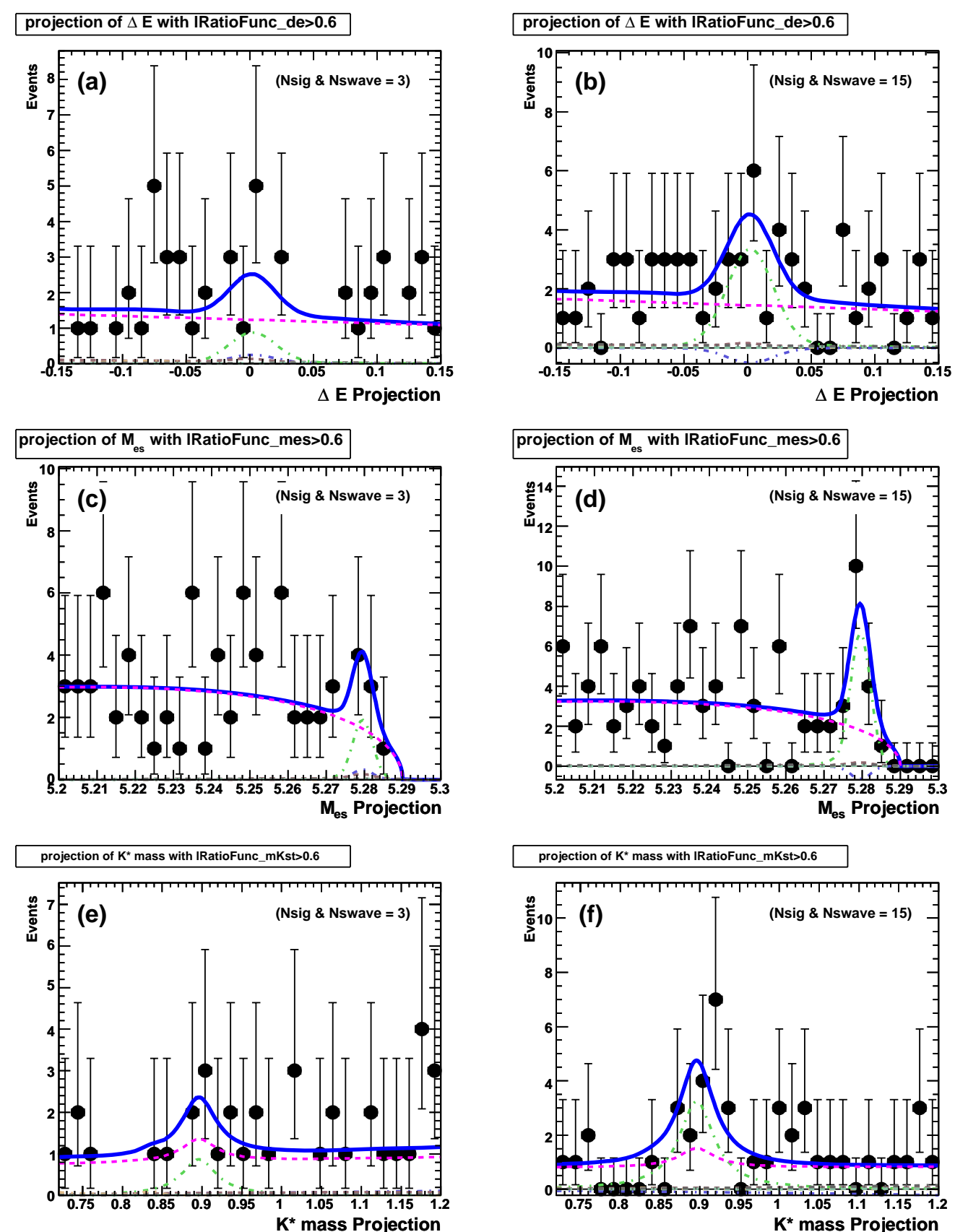

Figure 3.41: Projection plots for the three fitted parameters $\Delta E, m_{\mathrm{ES}}$, and the $K^{* 0}$ mass. Plots (a) and (b) show the $\Delta E$ projection plot with 3 and 15 expected signal events. Plots (c) and (d) show the $m_{\mathrm{ES}}$ projection plot with 3 and 15 expected signal events. Plots (e) and (f) show the $m_{K * 0}$ projection plot with 3 and 15 expected signal events. 
procedure works correctly, the two plots should be in rough agreement.

As a final test of the validity of the fit procedure, a blind fit was performed. The $210 \mathrm{fb}^{-1}$ of on-resonance data were used in the fit, but the fitted yields were masked by adding random numbers to the results. The fit procedure can be tested by comparing the errors in the fitted parameters and the global correlation coefficients that result from the blind fit with those obtained from toy studies. The global correlation coefficient is a measure of the strongest correlation between a variable and a linear combination of the other variables in the fit and is defined by

$$
\rho_{i}=\sqrt{1-1 /\left[C_{i i}\left(C_{i i}^{-1}\right)\right]}
$$

where $C_{i i}$ and $C_{i i}^{-1}$ are the diagonal elements of the covariance matrix and its inverse, respectively.

If the fit procedure works correctly, the blind fit errors and global correlations should fall generally within the envelope of the errors and global correlations obtained from the toy studies. Figures 3.43 and 3.44 show the results from the blind fit. The arrows indicate the blind fit values and show both the positive error and the absolute value of the negative error when asymmetric errors were obtained. All of the errors in the fitted parameters and correlations between the fitted parameters were generally within the range of values obtained in the toy studies. 

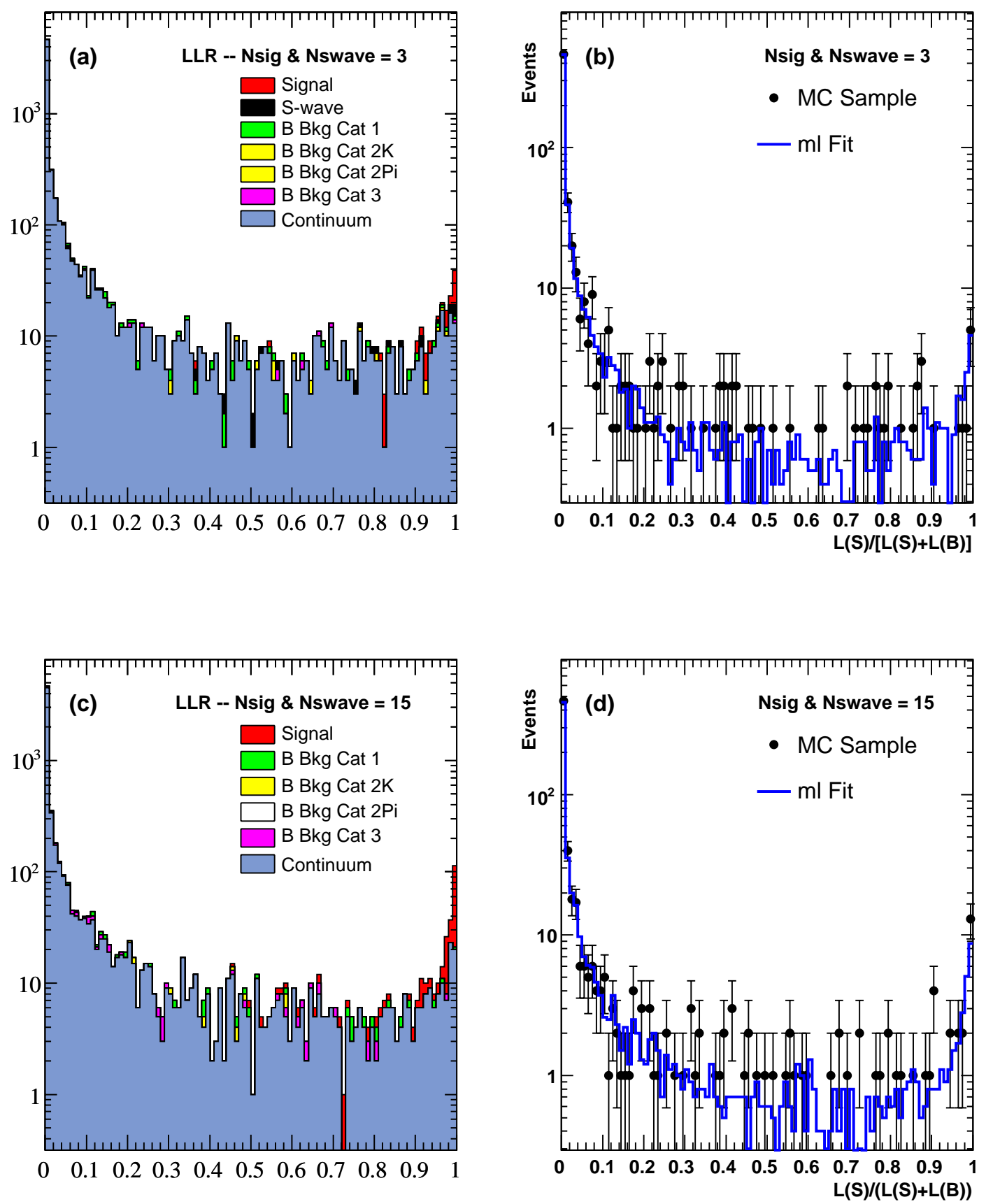

Figure 3.42: Plots of the log likelihood functions for the component PDFs and the product PDF and MC sample. Plots (a) and (c) show the component PDFs with 3 and 15 expected signal events respectively. Plots (b) and (d) show the product PDF the MC sample with 3 and 15 expected signal events respectively. 

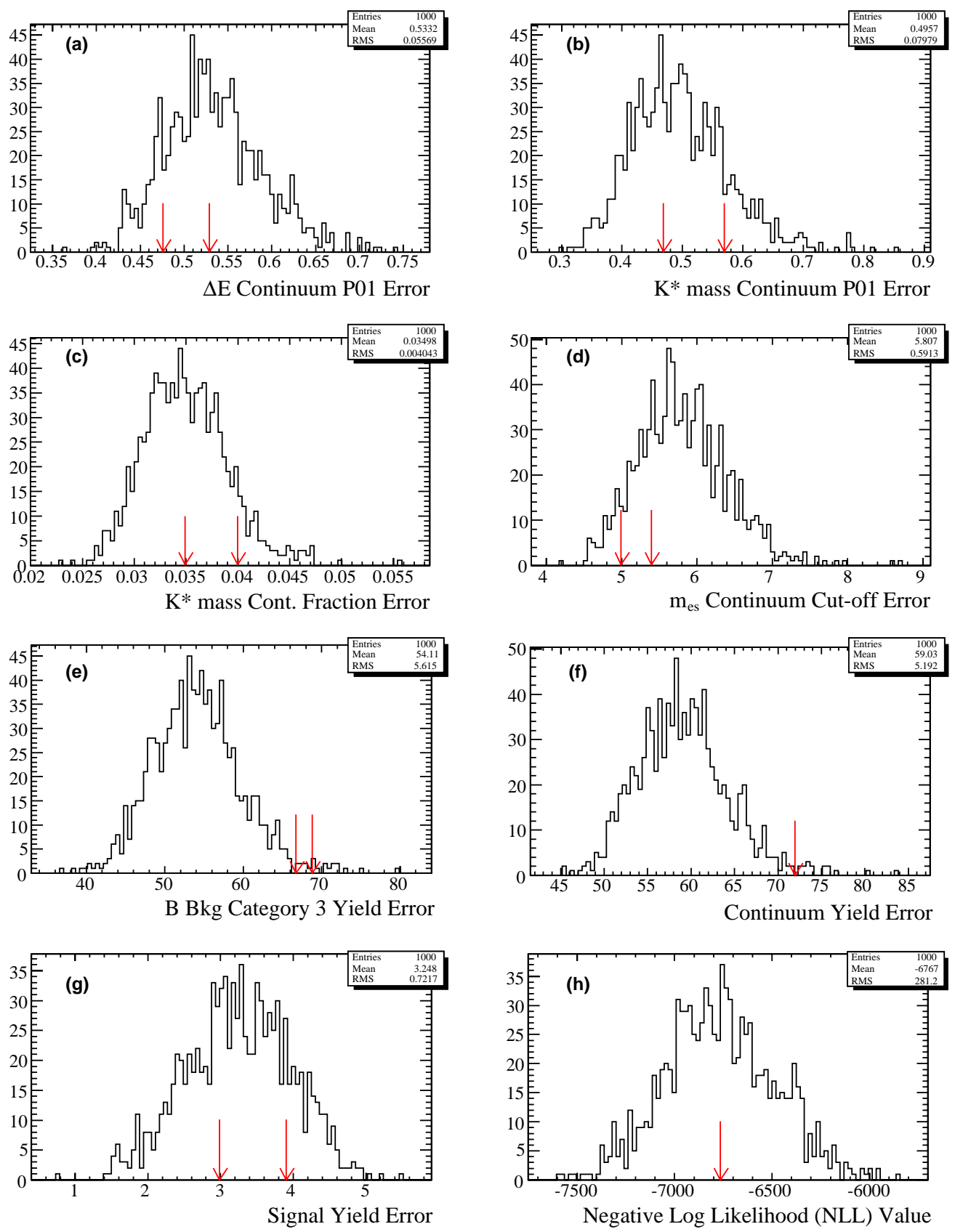

Figure 3.43: Plots (a) through (g) compare the distributions of the error for each of the fitted parameters obtained from toy studies (histograms) with the positive and absolute value of the negative error obtained from the blind fit (red arrows). Plot (h) compares the distribution of the minimum Negative Log Likelihood (NLL) value from the toy studies (histogram) with that obtained in the blind fit (red arrow). 

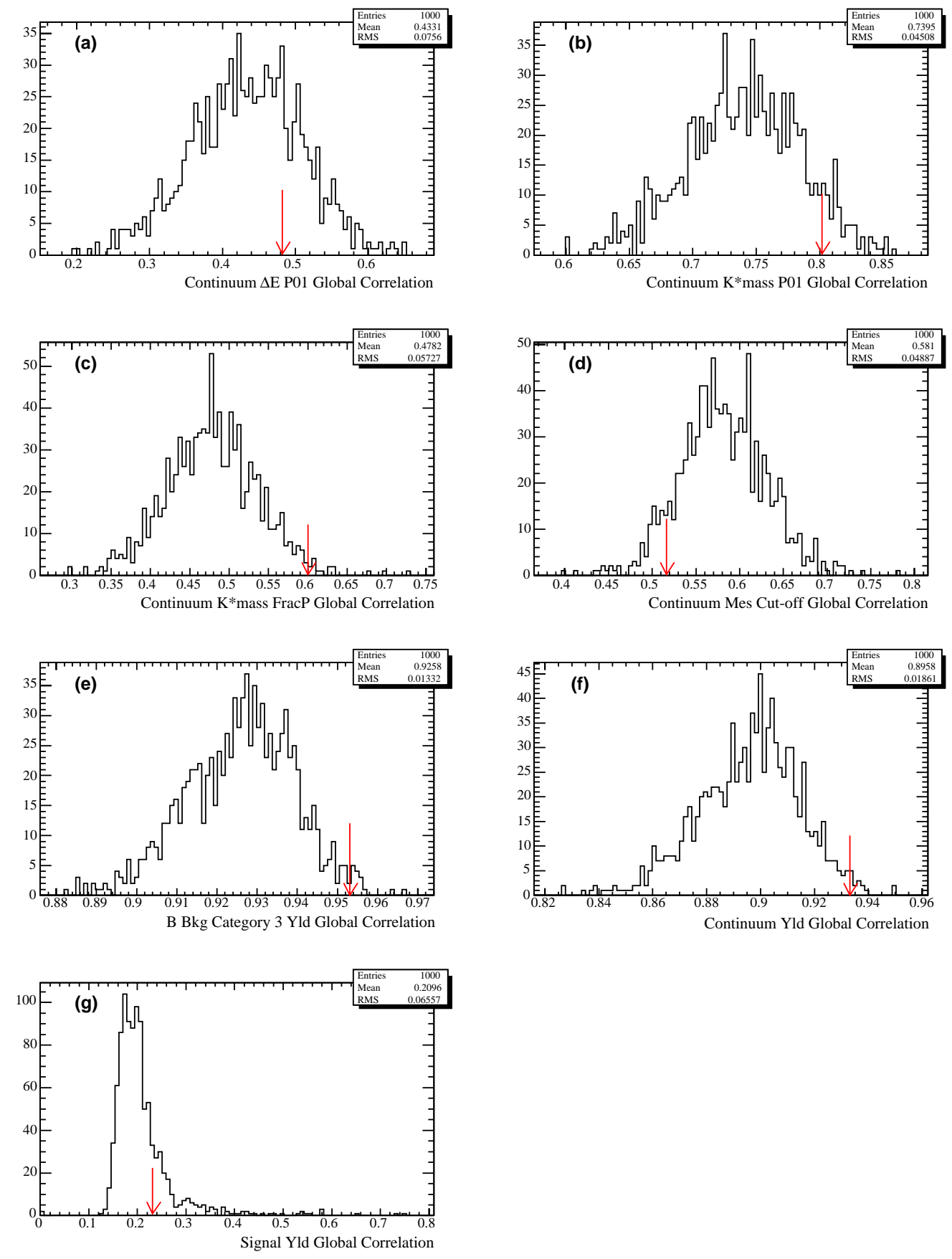

Figure 3.44: Plots (a) through (g) compare the distributions of the global correlations for each of the fitted parameters obtained from toy studies (histograms) with the global correlations obtained from the blind fit (red arrows). 


\section{Chapter 4}

\section{Results}

\subsection{Maximum Likelihood Fit Results}

The branching fraction is calculated from the following equation:

$$
\mathcal{B}\left(B^{0} \rightarrow K^{* 0} K^{0}\right)=\frac{N_{\text {sig }}}{\epsilon N_{B^{0} \bar{B}^{0}}},
$$

where $\epsilon$ is the signal efficiency after applying all necessary corrections. The sum of the two branching ratios $\mathcal{B}\left(B^{0} \rightarrow \bar{K}^{* 0} K^{0}\right)+\mathcal{B}\left(B^{0} \rightarrow K^{* 0} \bar{K}^{0}\right)$ was determined to be $\left(0.23_{-0.83}^{+0.94}{ }_{-0.31}^{+0.12}\right) \times 10^{-6}$ with a $90 \%$ confidence limit (CL) upper bound of $<1.9 \times 10^{-6}$. A summary of the results and the corrections applied are presented in Table 4.1.

The overall results of the fit are shown on several plots of the data. Figure 4.1 shows projections of $\Delta E, m_{\mathrm{ES}}$, and $m_{K^{* 0}}$ using subsets of the data with cuts on the likelihood ratio which enhance the signal to background ratio. Figure 4.2 shows two 
Table 4.1: Results.

\begin{tabular}{lc}
\hline Parameter & Value \\
\hline & \\
Number of Events Fit & 682 \\
Signal Yield & $1.0 \pm 4.3$ \\
Continuum Yield & $660 \pm 75$ \\
S-wave Background Yield & $1.4 \pm 5.9$ \\
B Background Category 4 Yield & $17 \pm 72$ \\
ML Fit Bias & -0.18 \\
Signal Efficiency & \\
after PID Tweaking & $9.76 \%$ \\
Efficiency Corrections & \\
$\quad K_{S}^{0}$ Tracking & $97.8 \%$ \\
$K^{* 0}$ Tracking & $99.0 \%$ \\
$\quad$ Final State $\mathcal{B}$ 's & $22.98 \%$ \\
Corrected Efficiency & $2.17 \%$ \\
\hline $\mathcal{B}\left(B^{0} \rightarrow \bar{K}^{* 0} K^{0}\right)+\mathcal{B}\left(B^{0} \rightarrow K^{* 0} \bar{K}^{0}\right)$ & $0.26 \sigma$ \\
Statistical Significance & $1.9 \times 10^{-6}$ \\
Significance with systematics & $\left(0.23_{-0.83}^{+0.94}{ }_{-0.31}^{+0.12}\right) \times 10^{-6}$ \\
$90 \%$ CL $\mathcal{B}\left(B^{0} \rightarrow \bar{K}^{* 0} K^{0}\right)+\mathcal{B}\left(B^{0} \rightarrow K^{* 0} \bar{K}^{0}\right)$ & $0.28 \sigma$ \\
\hline
\end{tabular}


plots of the likelihood ratio $\mathcal{L}(\mathrm{S}) /[\mathcal{L}(\mathrm{S})+\mathcal{L}(\mathrm{B})]$ for the data computed using the $\mathrm{ML}$ fit model.

Figure 4.3 shows a plot of $-2 \log \left(\mathcal{L} / \mathcal{L}_{0}\right)$ versus the branching fraction (NLL curve) for the data, where $\mathcal{L}_{0}$ is the likelihood minimum obtained from the original fit. The plot was generated by repeatedly re-fitting the data with a fixed number of signal events (or equivalently a fixed branching ratio). The other parameters were either fixed or floated in the same manner as the original fit used to extract the signal yield. Fifty different points with branching ratios between 0 and $4 \times 10^{-6}$ were used to generate the curves in fig. 4.3 .

The solid blue curve in fig. 4.3 was generated using the method described in Appendix G of [45] to broaden the NLL curve to include systematic uncertainties. This method adjusts the NLL curve by a factor defined by

$$
\chi^{2}=\frac{\chi_{\text {stat }}^{2} \chi_{\text {syst }}^{2}}{\chi_{\text {stat }}^{2}+\chi_{\text {syst }}^{2}}
$$

where $\chi_{\text {stat }}^{2}=(x-\mu)^{2} / \sigma_{\text {stat }}^{2}$ and similarly for $\chi_{\text {syst }}^{2}$ and $x$ are the individual data points on the curve, $\mu$ is the mean, and $\sigma_{\text {stat }}$ is the width.

The probability density function of the branching ratio defines the likelihood curve. The likelihood curve is derived by numerically inverting the NLL curve shown in fig. 4.3 to obtain $\mathcal{L}$ as a function of the branching ratio. The $90 \%$ confidence level (CL) upper limit for the branching fraction is defined as that point on the likelihood curve where the integral from 0 to that point equals $90 \%$ of the total area under the 
likelihood curve.

It is necessary to correct the signal yield to account for potential bias introduced by the ML procedure (see 3.8). To evaluate this bias, we applied the ML fit to 250 simulated data samples constructed as described below. The number of continuum background events in each sample was derived from a Poissonian distribution, with a mean set equal to the number of continuum events found in the fit to the data (i.e. 660 events). We generated $\Delta E, m_{\mathrm{ES}}$, and $m_{K^{* 0}}$ continuum distributions for each sample by randomly sampling the continuum PDFs using the appropriate number of events for each sample. The number of $B \bar{B}$ background events in each sample was determined in the analogous manner for each of the five $B \bar{B}$ background categories separately. For the first four categories of $B \bar{B}$ background (all but the scalar $K \pi$ component), the $\Delta E, m_{\mathrm{ES}}$, and $m_{K^{* 0}}$ distributions were generated by randomly selecting the appropriate number of events from the corresponding $B \bar{B} \mathrm{MC}$ sample. For the scalar $K \pi$ component, the distributions were generated by sampling the PDFs.

The number of signal events in each simulated sample was likewise determined from a Poissonian distribution, with a mean, $N_{\text {sig }}^{P}$, initially set equal to the observed signal yield, $N_{s i g}^{o b s}=1.0$. The signal $\Delta E, m_{\mathrm{ES}}$, and $m_{K^{* 0}}$ distributions were generated by randomly electing the appropriate number of signal $\mathrm{MC}$ events for each sample. $N_{\text {sig }}^{P}$ was then adjusted until the mean signal yield from the 250 samples equaled $N_{s i g}^{o b s}$. The ML fit bias was defined by $N_{s i g}^{o b s}-N_{s i g}^{P}$ and was determined to be -0.18 events. Therefore, the corrected signal yield is $N_{\text {sig }}=1.18$ events. 

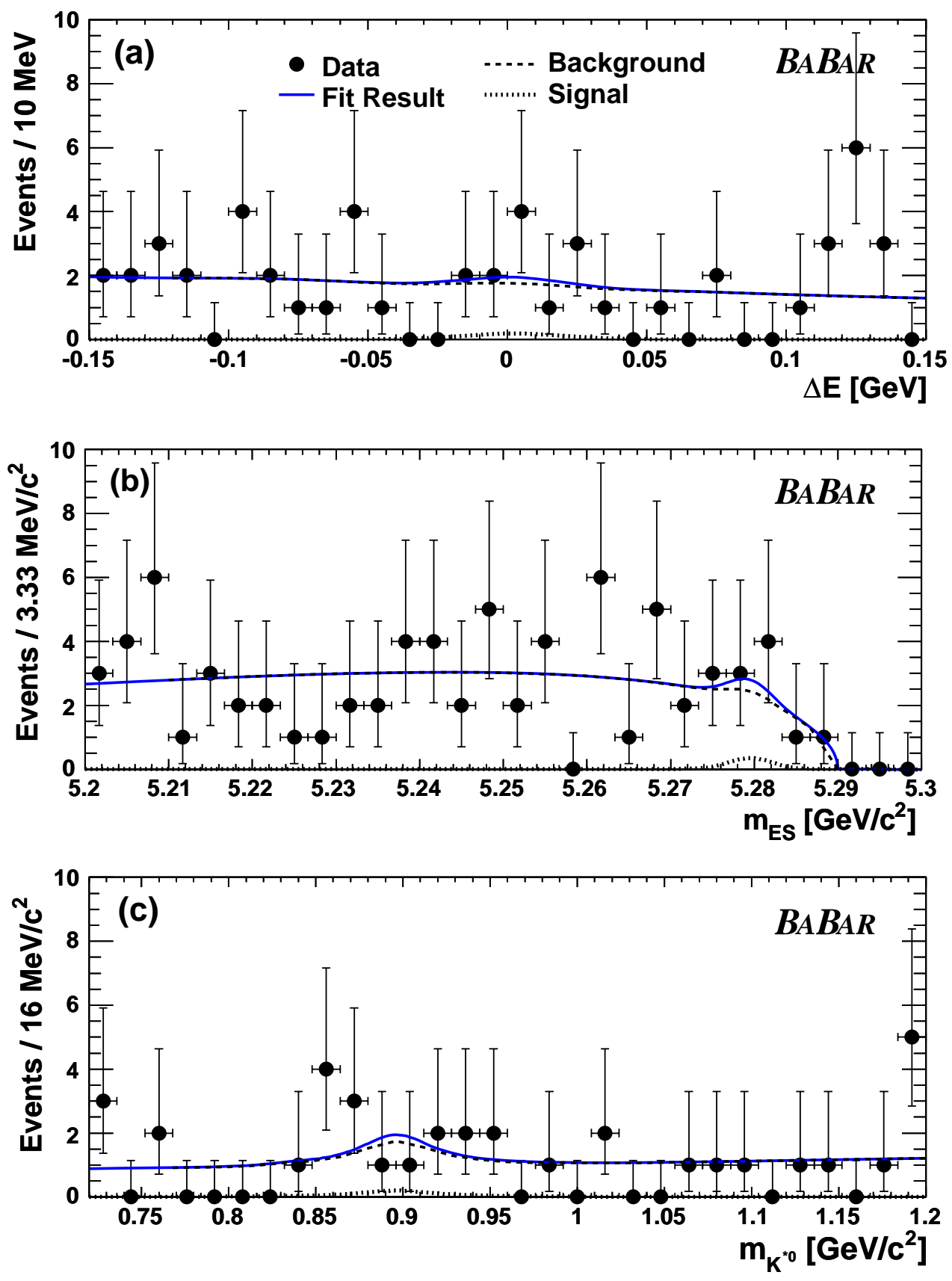

Figure 4.1: Projection plots for the three fitted variables $\Delta E, m_{\mathrm{ES}}$, and $m_{K^{* 0}}$. A cut on the likelihood ratio of $>0.6$ was included in all three plots. 

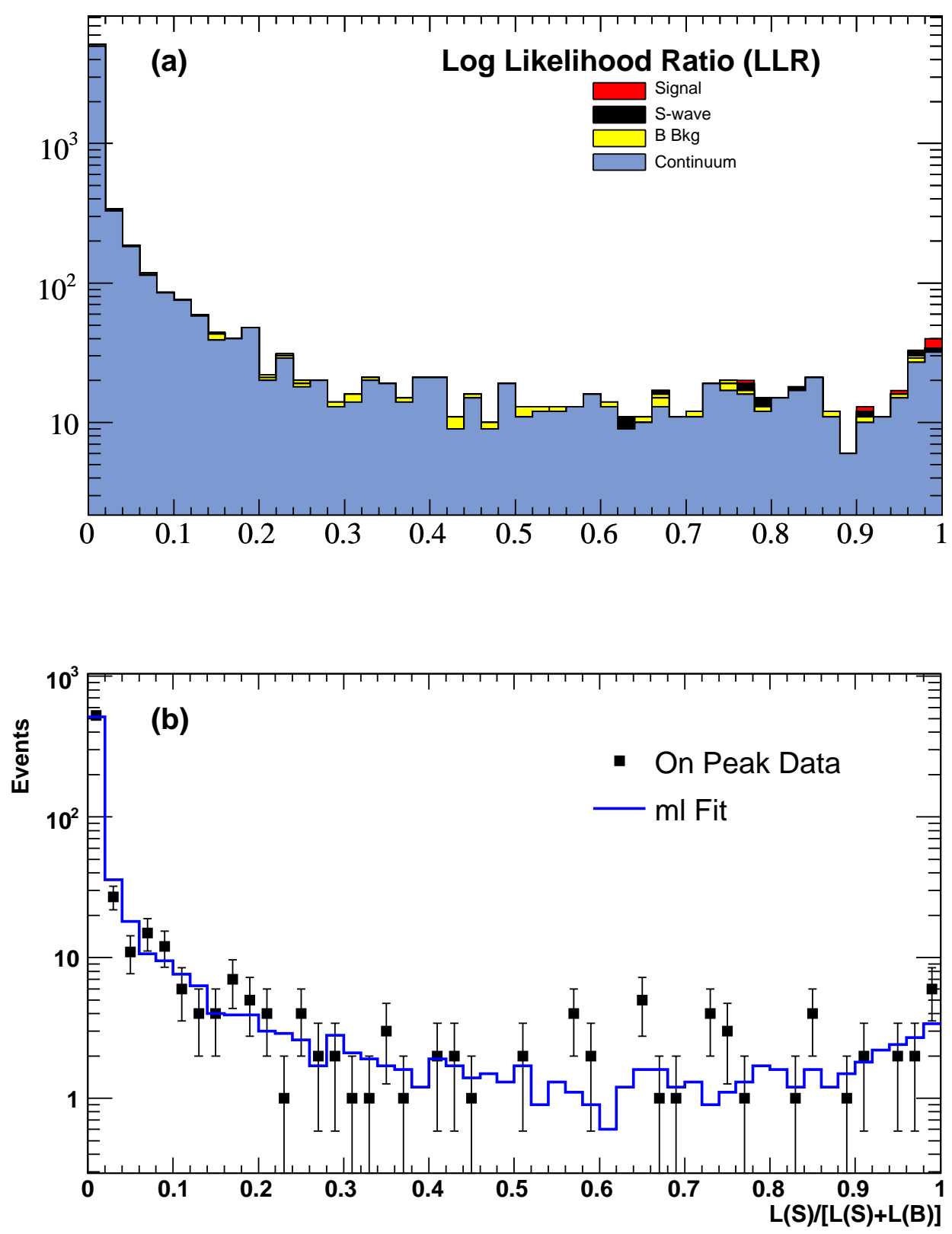

Figure 4.2: Log Likelihood Ratio (LLR) plots. Plot (a) shows the LLR for each component PDF and plot (b) shows the LLR for the total PDF and the data sample. 


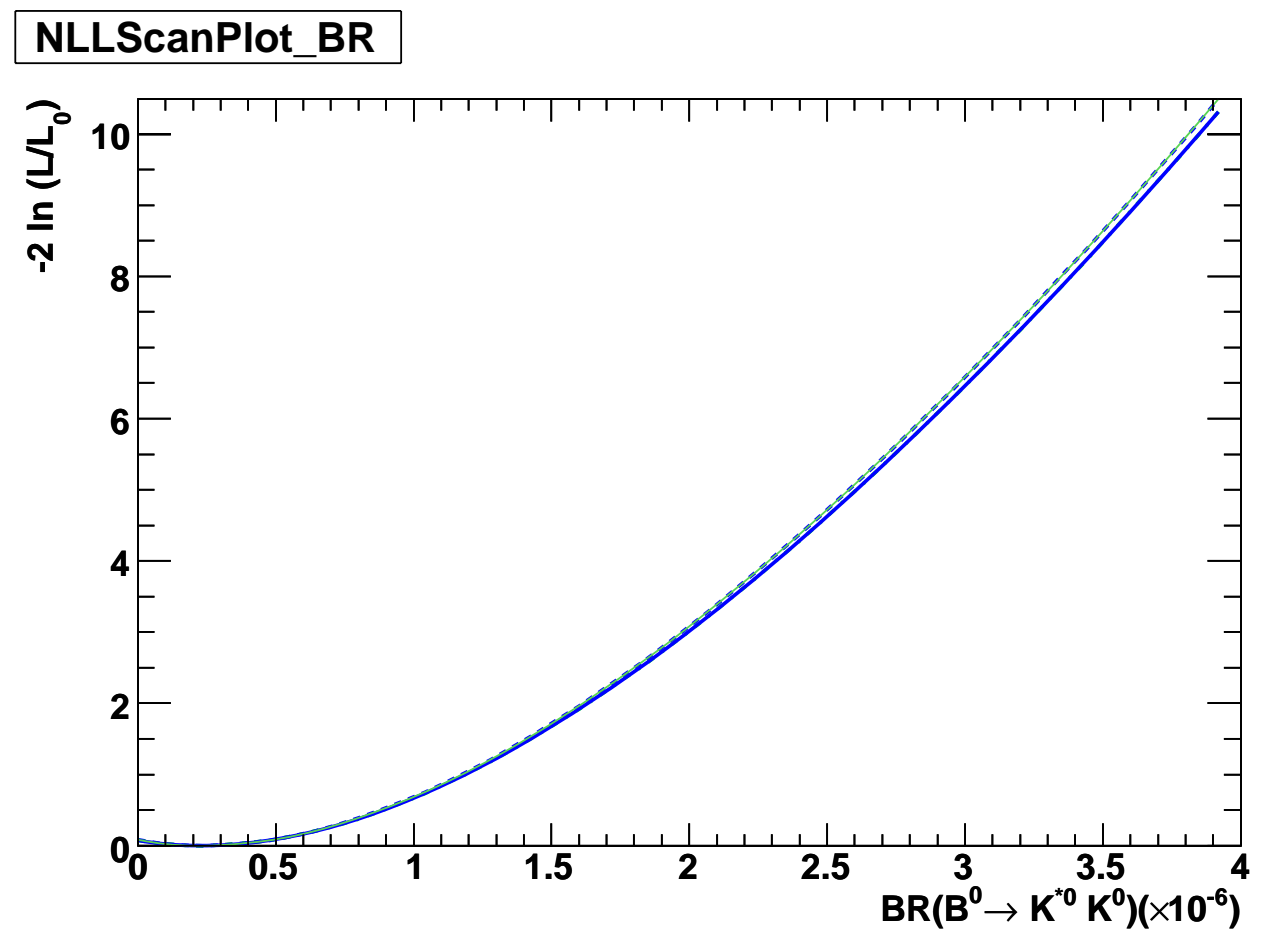

Figure 4.3: $-2 \log \left(\mathcal{L} / \mathcal{L}_{1}\right)$ versus the branching fraction. The dashed green curve is based on statistical uncertainties only, while the solid blue curve includes both statistical and systematic uncertainties. 


\subsection{Systematic Uncertainty Estimation}

The systematic uncertainties were divided into three broad categories: 1) uncertainties associated with the ML fit procedure, 2) uncertainties associated with efficiency corrections applied to the results, and 3) general uncertainties related to B counting and the branching ratios used in the final result.

\subsubsection{Fit Procedure Uncertainties}

To estimate the systematic uncertainty related to the signal PDFs, the 11 parameters used to characterize the signal $\Delta E, m_{\mathrm{ES}}$, and $m_{K^{* 0}}$ PDFs were independently varied. The mean and standard deviation of the central $\Delta E$ Gaussian, and the mean of the $m_{\mathrm{ES}}$ Crystal Ball function, were varied by the statistical uncertainties found by fitting the corresponding quantities to data in a recent study of $B^{0} \rightarrow \phi K^{0}[47]$. This channel is kinematically similar to the $K^{* 0} K^{0}$ channel studied here. The standard deviation of the $m_{\mathrm{ES}}$ Crystal Ball function was varied by an amount to account for observed variations between different run periods. The width of the $m_{K^{* 0}}$ Breit Wigner function was varied by a conservative $\pm 0.01 \mathrm{GeV} / c^{2}$. The remaining six signal PDF parameters were varied by one standard deviation of their statistical uncertainties found in the fits to the MC distributions (Sect. 3.7.2). For variations of all 11 parameters, the percentage change in the signal yield compared to the standard fit was taken as that parameter's contribution to the overall uncertainty. The total systematic uncertainty due to the signal PDFs was obtained by adding these 11 
contributions in quadrature. The largest contributions were from variations of the $\Delta E$ mean and standard deviation (about 0.3 signal events each). Table 4.2 shows the results for each Signal PDF parameter.

In addition to the above systematic uncertainties, it is necessary to estimate the systematic uncertainty in the fit bias (Sec. 3.8, Fig. 3.31) which was calculated using the method described in section 4.1. To estimate the systematic uncertainty of the bias due to the oversampling of the B background Category $4 \mathrm{MC}$ events, the bias was calculated using a second embedded Toy study where the B background Category 4 events were generated from the PDF and not embedded. The systematic uncertainty was taken as the difference between the bias calculated using these two toy studies.

To estimate the systematic uncertainty related to the lineshape of a possible scalar $K \pi$ component, the data was also fitted assuming the extreme possibility of a uniform distribution for the S-wave $m_{K * 0}$ PDF. The difference between the fitted yields was taken as the systematic uncertainty. This was taken as a one-sided systematic uncertainty.

Previous studies of charmless two-body decays [46] have determined that MC does not accurately model the $\cos \left(\theta_{B^{0}}\right)$ variable used in this analysis. It was found that the MC overestimates the number of selected events compared to data when the selection criterion for $\cos \left(\theta_{B^{0}}\right)$ is tighter than $<0.90$ and this overestimation increases as the selection criterion is tightened. For the cut used in this analysis $\left(\cos \left(\theta_{B^{0}}\right)<0.55\right)$, the ratio of $\mathrm{MC} /$ data was found to be $1.038 \pm 0.02$. There are, 
Table 4.2: Percentage systematic uncertainties from varying the Signal PDF parameters and the B background yields that were fixed in the ML fit.

\begin{tabular}{|l|c|c|c|}
\hline Parameter & Default Value & Range & $\Delta$ Signal Events \\
\hline Default Yield & 1.0 & & \\
\hline Signal Yield PDF Parameters & & & \\
$\Delta E$ PDF(2 Gaussians) & 0.00218 & $+/-0.0024$ & 0.287 \\
mean 1 & 0.0182 & $+/-0.0020$ & -0.261 \\
width 1 & 0.00228 & $+/-0.0113$ & -0.0381 \\
mean 2 & 0.0909 & $+/-0.0212$ & 0.0198 \\
width 2 & 0.892 & $+/-0.0213$ & -0.00856 \\
fraction 1 & 1.87 & $+/-0.179$ & -0.00974 \\
$m_{\text {ES PDF(Crystal Ball) }}$ & 5.28 & $+/-0.00034$ & -0.0781 \\
alpha & 2.12 & $+/-0.450$ & -0.0105 \\
mean & 0.00266 & $+0.00014-0.00021$ & 0.112 \\
n & & & 0.0014 \\
width & 0.896 & $+/-0.01$ & 0.176 \\
$m_{K^{* 0} \text { PDF(Breit-Wigner) }}$ & 0.0537 & $+/-0.00245$ & 0.450 \\
$\quad$ mean & & & -0.0441 \\
width & 1.0 & $+2-1$ & 0.0186 \\
Quadrature Sum & 1.0 & $+2-1$ & -0.0548 \\
\hline Fixed B Bkg Yields & 1.0 & & 0.0728 \\
Category 1 Yield & & & \\
Category 2 Yield & & & \\
Category 3 Yield & & & \\
Quadrature Sum & & & \\
\hline
\end{tabular}


however, strong correlations between the Fisher variable and the $\cos \left(\theta_{B^{0}}\right)$ variable which could affect these results (See Fig. 3.11). The variation in the Fisher was also studied by [46], but the Fisher used in that analysis contained other variables in addition to the Legendre moments.

It was concluded that the results in [46] are not sufficiently applicable to determine an efficiency correction to the signal yield. A systematic uncertainty was therefore applied to account for this effect. The $3.8 \%$ over estimation found in [46] was increased to a $5.0 \%$ systematic uncertainty to be conservative.

\subsubsection{Efficiency Correction Uncertainties}

Efficiency correction factors for $K_{S}^{0}$ efficiency, tracking efficiency, and PID must be applied to the results. The $K_{S}^{0}$ efficiency correction and the statistical and systematic uncertainties associated with this correction were calculated following the procedure described in [42] and are based on correction tables that have been developed for the $B A B A R$ experiment. Use of the correction tables requires four separate calculations due to different HV settings on the Drift Chamber and changes in the efficiency tables. The data groups were defined as follows:

- October through November 1999 (Run 1a)

- January through October 2000 (Run 1b)

- February 2001 through June 2003 (Runs 2 and 3)

- September 2003 through July 2004 (Run 4) 
Table 4.3: $K_{S}^{0}$ efficiency correction factors.

\begin{tabular}{|c|c|c|c|c|c|}
\hline Correction Table & Cut Combination & Run1a & Run1b & Runs $2 / 3$ & Run 4 \\
\hline 3DSign3_3DAlpha & $\begin{array}{l}K_{S}^{0} \text { decay length sig }>3 \\
\left|\cos \theta_{K_{S}^{0}}\right|>0.995\end{array}$ & $\begin{array}{c}0.953 \\
\pm 0.050\end{array}$ & $\begin{array}{c}1.011 \\
\pm 0.052\end{array}$ & $\begin{array}{c}0.968 \\
\pm 0.020\end{array}$ & $\begin{array}{c}0.973 \\
\pm 0.021\end{array}$ \\
\hline 3D1mm_3DAlpha & $3 \mathrm{D}$ flgt Dist $>1 \mathrm{~mm}$ & 0.919 & 0.997 & 0.965 & 0.972 \\
\hline 0.0_pvtx0.001 & $\begin{array}{l}\left|\cos \theta_{K_{S}^{0}}\right|>0.995 \\
K_{S}^{0} \text { vertex prob }>0.001\end{array}$ & \pm 0.047 & \pm 0.053 & \pm 0.024 & \pm 0.026 \\
\hline 3DSign3_noAlpha & $\begin{array}{l}K_{S}^{0} \text { decay length sig }>3 \\
\text { no Cut on Alpha }\end{array}$ & $\begin{array}{c}0.901 \\
\pm 0.046\end{array}$ & $\begin{array}{c}0.990 \\
\pm 0.053\end{array}$ & $\begin{array}{c}1.004 \\
\pm 0.031\end{array}$ & $\begin{array}{c}0.994 \\
\pm 0.025\end{array}$ \\
\hline Average & & $\begin{array}{c}0.924 \\
\pm 0.083\end{array}$ & $\begin{array}{c}0.999 \\
\pm 0.091\end{array}$ & $\begin{array}{c}0.979 \\
\pm 0.045\end{array}$ & $\begin{array}{c}0.980 \\
\pm 0.042\end{array}$ \\
\hline Luminosity $\mathrm{fb}^{-1}$ & & 10.468 & 8.990 & 91.327 & 99.762 \\
\hline Weighted Average & $0.978 \pm 0.032$ & & & & \\
\hline
\end{tabular}

In addition, none of the correction tables exactly matched the selection criteria used in this analysis. Consequently the three tables that most closely matched the criteria in this analysis were used and the results were averaged for each run. A luminosity weighted average of these factors was then used as the final $K_{S}^{0}$ efficiency correction factor and associated uncertainties. Table 4.3 shows the correction tables used and the resulting $K_{S}^{0}$ efficiency correction factors and uncertainties. The weighted average $K_{S}^{0}$ efficiency correction factor is $0.978 \pm 0.032$ with a statistical uncertainty of $2.9 \%$ and a systematic uncertainty of $1.4 \%$.

The tracking efficiency factor for the $K^{* 0}$ was determined using the findings of the BABAR Tracking Efficiency Task Force [41] based on an analysis of tau events. The "GoodTracksVeryLoose" efficiency correction factor is $0.5 \%$ per track with a 
systematic uncertainty of $1.4 \%$ per track for a total efficiency correction of 0.990 (2 tracks at $0.5 \%$ per track) with a systematic uncertainty of $2.8 \%$.

The PID efficiency correction was implemented by applying BABAR PID corrections (called "tweaking") to the kaon and pion selectors [43]. PID corrections involve an internal BABAR procedure for removing MC events to bring signal MC samples into agreement with data samples. The systematic uncertainty associated with this process is estimated to be $0.6 \%$ per kaon and $0.2 \%$ per pion based on studies using a control sample of $B^{0} \rightarrow J / \psi K^{ \pm}$[44]. For this analysis, PID was only applied to identify the kaon and pion used to reconstruct the $K^{* 0}$. Since kaon and pion PID are correlated and to be conservative, the systematic errors for the kaon and pion PID were added linearly which results in a total percentage systematic uncertainty of $0.8 \%$ for PID efficiency corrections.

\subsubsection{General Systematic Uncertainties and Summary}

The remaining systematic uncertainties are related to the number of $B^{0} \bar{B}^{0}$ pairs in the data sample and the branching fractions to the $K^{ \pm} \pi^{\mp} \pi^{ \pm} \pi^{\mp}$ final state. The number of $B^{0} \bar{B}^{0}$ pairs and the statistical and systematic uncertainties in this number were obtained using the BbkLumi function applied to the skimmed data sample. The method for determining the number of $B \bar{B}$ pairs is described in [51] and is based on measuring the total number of hadronic events and subtracting the non- $B \bar{B}$ events using an off-resonance sample. 
The BABAR luminosity is measured through the rates of wide angle $e^{+} e^{-} \rightarrow e^{+} e^{-}(\gamma)$ and $e^{+} e^{-} \rightarrow \mu^{+} \mu^{-}(\gamma)$ events. The luminosity is measured with a precision of about $1 \%[52]$

The branching fractions and their uncertainties were obtained from [9]. Table 4.4 shows all of the systematic uncertainties associated with the final result. 
Table 4.4: Summary of systematic uncertainties.

\begin{tabular}{|c|c|}
\hline Type & Uncertainty \\
\hline \multicolumn{2}{|l|}{ ML Fit Procedure (Events) } \\
\hline Signal PDF Parameters & 0.45 \\
\hline Background Yields & 0.07 \\
\hline Fit Bias & 0.41 \\
\hline Total ML Fit (Events) & 0.61 \\
\hline LASS Line Shape (Events) & ${ }_{-1.4}^{+0.0}$ \\
\hline Efficiency Corrections (\%) & \\
\hline$K_{S}^{0}$ Tracking & $1.4 \%$ \\
\hline$K^{* 0}$ Tracking & $2.8 \%$ \\
\hline PID Efficiency & $0.8 \%$ \\
\hline $\cos \left|\theta_{B^{0} \text {-thrust }}\right|$ cut & $5.0 \%$ \\
\hline$B^{0} \overline{B^{0}}$ pairs & $1.1 \%$ \\
\hline $\mathcal{B}\left(K_{S}^{0} \rightarrow \pi^{ \pm} \pi^{\mp}\right)$ & $0.14 \%$ \\
\hline Total Efficiency Corrections & $6.1 \%$ \\
\hline Total Errors $\left[\mathcal{B}\left(10^{-6}\right)\right]$ & ${ }_{-0.31}^{+0.12}$ \\
\hline
\end{tabular}




\section{Chapter 5}

\section{$\Delta S_{\phi K^{0}}$ Calculation}

As discussed in Section 1.1 the upper limit placed on the sum of the two branching fractions $\mathcal{B}\left(B^{0} \rightarrow \overline{K^{* 0}} K^{0}\right)$ and $\mathcal{B}\left(B^{0} \rightarrow K^{* 0} \overline{K^{0}}\right)$ allows the $\mathrm{SU}(3)$ upper bound on $\Delta S_{\phi K^{0}}$ to be calculated for the first time. Equation (1.38) requires either the separate measurement of $\mathcal{B}\left(B^{0} \rightarrow \overline{K^{* 0}} K^{0}\right)$ and $\mathcal{B}\left(B^{0} \rightarrow K^{* 0} \overline{K^{0}}\right)$ or the measurement of the coherent sum of the two amplitudes. Our results are for the sum of the two branching fractions. Equation (5.1) shows the relationship between the sum of the two branching fractions and the amplitudes assuming no direct $C P$ violation.

$$
\mathcal{B}=a_{1}^{2}+a_{2}^{2}
$$

Where $\mathcal{B}$ represents the sum of the two branching fractions $\mathcal{B}\left(B^{0} \rightarrow \overline{K^{* 0}} K^{0}\right)+$ $\mathcal{B}\left(B^{0} \rightarrow K^{* 0} \overline{K^{0}}\right)$ and $a_{1}$ and $a_{2}$ represent the two amplitudes. The sum $a_{1}+a_{2}$ can not be determined from the limit determined in this analysis, but for a given value 
of $\mathcal{B}$, the maximum value of $a_{1}+a_{2}$ occurs when $a_{1}=a_{2}$ in Eq. (5.1). Therefore the maximum value for the sum of the two amplitudes for a given value of $\mathcal{B}$ is

$$
a_{1}+a_{2}=\sqrt{2 \mathcal{B}}
$$

To incorporate our results into the calculation of $\Delta S_{\phi K^{0}}$, the first term in square brackets in Eq. (1.38) must be replaced with the square root of 2 times the value measured for the sum of the two branching fractions.

Table 5.1 shows the current values for all of the branching fractions [48, 49] required in Eq. (1.38) and their contributions to $\Delta S_{\phi K^{0}}$ along with the contribution from $\overline{K^{* 0}} K^{0}$ and $K^{* 0} \overline{K^{0}}$. A $90 \%$ CL upper limit on $\left|\Delta S_{\phi K^{0}}\right|$ was evaluated by generating hypothetical sets of branching ratios for the 11 required SU(3)-related decays shown in Table 5.1. Branching ratio values were chosen using bifurcated Gaussian probability distribution functions, with means and bifurcated widths set equal to the measured branching ratios and the asymmetric uncertainties (for the measurements of the branching rations of the nine channels not included in the present study, see $[48,49])$. Negative generated branching ratios were discarded. For each set of hypothetical branching ratios, we computed a bound on $\left|\Delta S_{\phi K^{0}}\right|$ using Eqs. (1.38) and (1.35). For the unknown phase term $\cos \delta$ in Eq. (1.35), we sampled a uniform distribution between -1 and 1 . Similarly, the weak phase angle $\gamma$ was chosen by selecting values from a uniform distribution between 44 and 74 degrees, corresponding to the 95\% confidence level interval for $\gamma$ given in [50] (a flat distribution is choses for $\gamma$ 
Table 5.1: Contributions to the calculation of $\Delta S_{\phi K^{0}}$. The branching ratios and upper limits are from $[48,49]$

\begin{tabular}{lrcc}
\hline & & $\mathcal{B}$ Upper Limit & $\Delta$ S \\
Mode & $\mathcal{B} \times 10^{-6}$ & $90 \%$ CL $\times 10^{-6}$ & Contribution \\
\hline$\phi \eta$ & $-1.4_{-0.45}^{+0.73}$ & $<1.0$ & 0.054 \\
$\phi \eta^{\prime}$ & $1.5_{-1.55}^{+1.84}$ & $<4.5$ & 0.043 \\
$\omega \eta$ & $1.0_{-0.54}^{+0.54}$ & $<1.9$ & 0.053 \\
$\omega \eta \prime$ & $-0.2_{-0.98}^{+1.36}$ & $<2.8$ & 0.024 \\
$\rho^{0} \eta$ & $-1.1_{-0.98}^{+0.81}$ & $<1.5$ & 0.047 \\
$\rho^{0} \eta^{\prime}$ & $0.8_{-1.5}^{+1.92}$ & $<4.3$ & 0.030 \\
$\rho \pi^{0}$ & $1.83_{-0.6}^{+0.6}$ & $<2.6$ & 0.032 \\
$\omega \pi^{0}$ & $-0.6_{-0.54}^{+0.73}$ & $<1.2$ & 0.026 \\
$\phi \pi^{0}$ & $0.2_{-0.32}^{+0.41}$ & $<1.0$ & 0.033 \\
Subtotal & & & 0.32 \\
\hline $\bar{K}^{* 0} K^{0}+K^{* 0} \bar{K}^{0}$ & $0.2_{-0.88}^{+0.95}$ & $<1.9$ & 0.093 \\
Total & & & 0.43 \\
\hline
\end{tabular}


because the likelihood curve [50] is non-Gaussian). For $\beta$, we used $\sin 2 \beta=0.687$ [49]. For each iteration of variables, Eq. (1.39) was solved numerically for $\left|\xi_{\phi K^{0}}\right|$.

We found that $90 \%$ of the hypothetical $\left|\Delta S_{\phi K^{0}}\right|$ bounds were below 0.43 . The contribution of the $K^{* 0} K^{0}$ measurement to this value is 0.09 . This study thus allows an $\mathrm{SU}(3)$ bound [15] on $\Delta S_{\phi K^{0}}$, viz. $\left|\Delta S_{\phi K^{0}}\right|<0.43$ at $90 \%$ CL, to be determined for the first time. This analysis does not account for SU(3) flavor symmetry breaking effects, expected to be on the order of $30 \%$. However, the method is conservative in that it assumes all hadronic amplitudes to interfere constructively.

None of the 11 decay channels required for this $\Delta S_{\phi K^{0}}$ bound have been observed, although evidence for $B^{0} \rightarrow \rho^{0} \pi^{0}$ has been reported [49]. As improved upper limits or observations of the 11 decay channels become available, this bound on $\Delta S_{\phi K^{0}}$ should become more restrictive. If all of the decay channels are measured to be their theoretically predicted values, the upper limit on $\Delta S_{\phi K^{0}}$ would be 0.14 . 


\section{Bibliography}

[1] A. Ali, G. Kramer, and C-D Lü, Phys. Rev. D58, 094009 (1998).

[2] N.G. Deshpande, B. Dutta, and Sechul Oh, Phys. Lett. B473, 141 (2000).

[3] Y-H Chen, H-Y Cheng, B. Tseng, and K-C Yang, Phys. Rev. D60, 094014 (1999).

[4] M. Beneke and M. Neubert, Nucl. Phys. B675, 333 (2003).

[5] C-W. Chiang et al., Phys. Rev. D69, 034001 (2004).

[6] R. Wang, G.R. Lu, E-K Wang and Y-D Yang, hep-ph/0603088 (2006).

[7] M. Kobayashi and T. Maskawa, Prog. Theor. Phys., 49, 652 (1973).

[8] M. Peskin, and D. Schroeder, An Introduction to Quantum Field Theory Westview Press, 1995, eq.(21.71).

[9] S. Eidelman et al., Phys. Lett. B592, 1 (2004).

[10] L.-L. Chau and Y.-Y. Keung, Phys. Rev. Lett. 53, 1802 (1984); H. Harari and M. Leurer, Phys. Lett. B181, 123 (1986); H. Fritzsch and J. Plankl, Phys. Rev. D35, 1732 (1987); F.J. Botella dnd L.-L. Chao, Phys. Lett. B168, 97 (1986).

[11] L. Wolfenstein, Phys. Rev. Lett.,51, 1945 (1983).

[12] N. Cabibbo, Phys. Rev. Lett. 10, 531 (1963).

[13] See, for example, the results on the Unitarity Triangle given at http://www.slac.stanford.edu/xorg/hfag.

[14] M. Gronau, Phys. Rev. Lett. 63, 1451 (1989).

[15] Y. Grossman, Z. Ligeti, Y. Nir, and H. Quinn, Phys. Rev. D68, 015004 (2003). 
[16] Y. Grossman and M. Worah, Phys. Lett. B395, 241-249 (1997).

[17] Y. Grossman, G. Isidori and M.P. Worah, Phys. Rev. D 58, 057504 (1998).

[18] G. Engelhard, Y. Nir, and G. Raz, Phys. Rev. D70, 032006 (2004).

[19] J. Seeman, et al., SLAC-PUB-11727 (May 2005).

[20] D. Lange, Nucl. Instrum. and Meth., A462, 152 (2001).

[21] B. Aubert et al. (BABAR Collaboration), Nucl. Instrum. Meth. A479, 1-116 (2002).

[22] F. Anulli et al., Nucl. Instrum. Meth. A539, 155-171 (2005).

[23] S. Foulkes, J.W. Gary, B.C. Shen, K.Wang, R. Boyce, R. Messner, P. Stiles, N. Sinev, H.R. Band, Nucl. Instrum. Meth. A538, 801-809 (2005).

[24] G. Lutz and A.S. Schwartz, Annu. Rev. Nucl. Part. Sci., 45, 295-335 (1995).

[25] D. Bailey and R. Hall-Wilton, Nucl. Instrum. Meth. A515, 37-42 (2003).

[26] L. Malter, Phys.Rev 5048 (1936).

[27] S.J. Bailey et al., EEE 2003 Nucler Science Symposium and Medical Imaging Conference (Oct 2003) (SLAC-PUB-10461).

[28] http://www.slac.stanford.edu/BFROOT/www/Physics/Tools/Pid/Hadrons/ Description_of_the_LH_selectors.html.

[29] T. Latham, University of Bristol, PhD Thesis (2005).

[30] H. Haber, hep-ph/9405376 (1994).

[31] T. Brandt, BABAR Analysis Document 391.

[32] S. Brandt et al., Phys. Lett. 12, 57 (1964);

E. Fahri, Phys. Rev. Lett. 39, 1587 (1977).

[33] T. Skwarnicki et al. (Crystal Ball Collaboration), DESY Report DESY-F31-8602, 1986 (unpublished).

[34] H. Albrecht et al. (ARGUS Collaboration), Z. Phys. C48, 543 (1990).

[35] C. Campagnari, O. Long, and W. Verkerke, BABAR Analysis Document 728, p. 27.

[36] T.E. Latham and S.E. Morgan, BABAR Analysis Document 1181. 
[37] D. Aston et al. (LASS Collaboration), Nucl. Phys. B296, 493 (1988).

[38] W.M. Dunwoodie, private communication.

[39] BELLE Collab, A. Garmash et al., Phys. Rev. D71, 092003 (2005).

[40] http://www.slac.stanford.edu/ zhanglei/RooRarFit.

[41] http://www.slac.stanford.edu/BFROOT/www/Physics/TrackEfficTaskForce/ TrackingTaskForce-2004.html.

[42] http://www.slac.stanford.edu/BFROOT/www/Physics/TrackEfficTaskForce/ Recipe/R12/KsEff_R12.html; http://www.slac.stanford.edu/BFROOT/www/Physics/TrackEfficTaskForce/ Recipe/R14/KsEff_R14.html.

[43] http://www.slac.stanford.edu/BFROOT/www/Physics/Tools/Pid/PidOnMc/ pidonmc.html.

[44] http://www.slac.stanford.edu/BFROOT/www/Organization/Workshops/2005/ bbr_analtools/Tools05.html.

[45] S.J. Bailey et al., BABAR Analysis Document 521.

[46] P. Bloom, P.J. Clark, W. Gradl, and A.I. Robertson, BABAR Analysis Document 960.

[47] BABAR Collab., B. Aubert et al., Phys. Rev. D71, 091102 (2005).

[48] B. Aubert, et al. (BABAR Collaboration), Phys. Rev. Lett. 95, 181806 (2004); 95, 131803 (2005); Phys. Rev. D70, 032006 (2004).

[49] E. Barberio et al., (Heavy Flavor Averaging Group), hep-ex/0603003.

[50] J. Charles et al. (CKMfitter Group), Eur. Phys. J.C 41, 1 (2005).

[51] C. Hearty, BABAR Analysis Document 30v5 and BABAR Analysis Document $134 \mathrm{v} 1$. For a summary of the package see http://www.slac.stanford.edu/BFROOT/www/Computing/Distributed/ Bookkeeping/Documentation/package_docs/BbkLumi_help.html.

[52] R. Gamet and C. Touramanis, BABAR Analysis Document 1312v1. 